\title{
Sufficient conditions for
}

\section{hamiltonian properties of graphs}

\section{Qiannan Zhou}
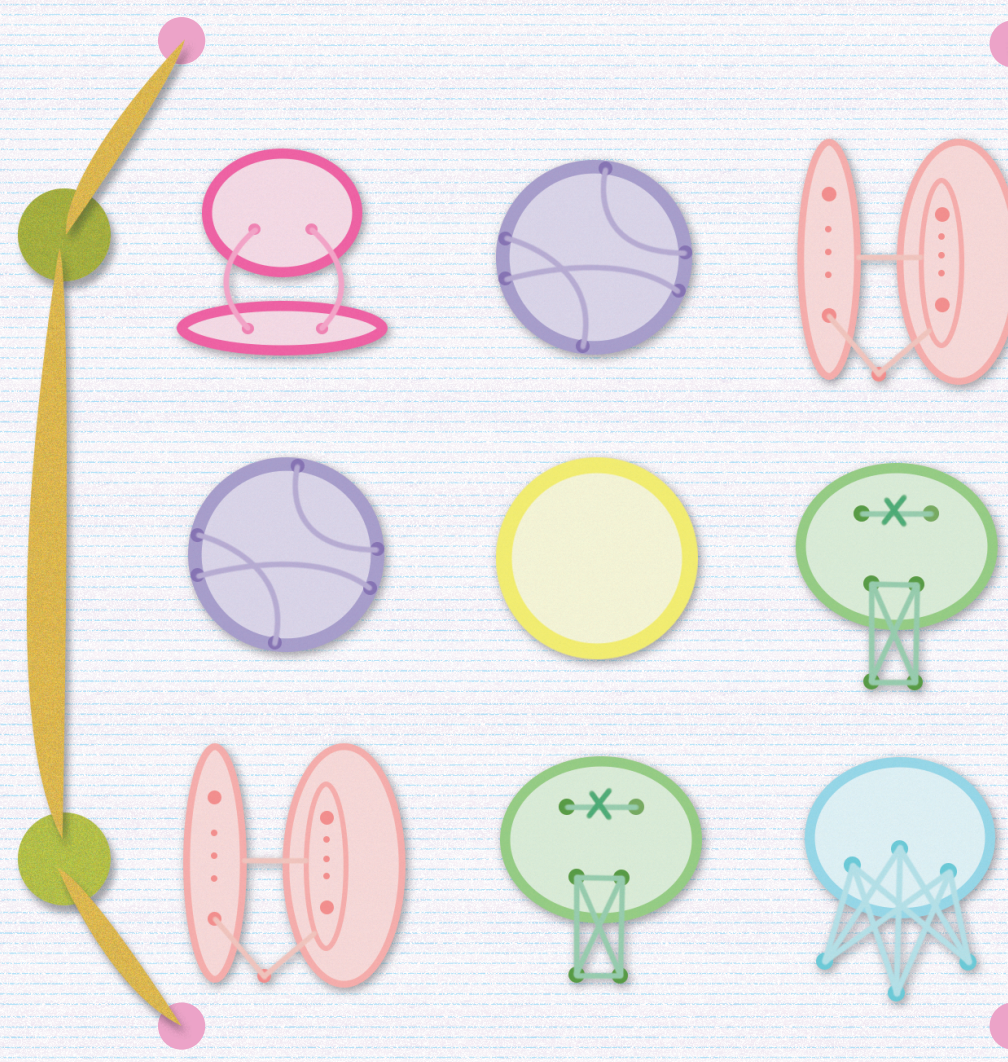



\section{SUFFICIENT CONDITIONS FOR \\ HAMILTONIAN PROPERTIES OF GRAPHS}

Qiannan Zhou 



\title{
SUFFICIENT CONDITIONS FOR HAMILTONIAN PROPERTIES OF GRAPHS
}

\author{
DISSERTATION
}

to obtain

the degree of doctor at the University of Twente, on the authority of the rector magnificus, prof.dr. T.T.M. Palstra, on account of the decision of the graduation committee, to be publicly defended on Thursday the $10^{\text {th }}$ of October 2019 at $12.45 \mathrm{hrs}$

by

Qiannan Zhou

born on the $27^{\text {th }}$ of November 1992 in Henan, China 
This dissertation has been approved by the supervisors prof. dr. ir. H.J. Broersma and prof. dr. L. Wang

The research reported in this thesis has been carried out within the framework of the MEMORANDUM OF AGREEMENT FOR A DOUBLE DOCTORATE DEGREE BETWEEN NORTHWESTERN POLYTECHNICAL UNIVERSITY, PEOPLE'S REPUBLIC OF CHINA AND THE UNIVERSITY OF TWENTE, THE NETHERLANDS

DSI Ph.D. Thesis Series No. 19-017

UNIVERSITY $\mid$ DIGITAL SOCIETY Digital Society Institute

OF TWENTE. INSTITUTE P.O. Box 217, 7500 AE Enschede, The Netherlands.

ISBN: 978-90-365-4864-9

ISSN: 2589-7721 (DSI Ph.D. thesis Series No. 19-017)

DOI: $10.3990 / 1.9789036548649$

Available online at https://doi.org/10.3990/1.9789036548649

Typeset with $\mathrm{BT}_{\mathrm{E}} \mathrm{X}$

Printed by Ipskamp Printing, Enschede

Cover design by Qiannan Zhou

Copyright (C)2019 Qiannan Zhou, Enschede, The Netherlands

All rights reserved. No part of this work may be reproduced, stored in a retrieval system, or transmitted in any form or by any means, electronic, mechanical, photocopying, recording, or otherwise, without prior permission from the copyright owner. 


\section{Graduation Committee}

Chairman/secretary:

prof. dr. J.N. Kok University of Twente

Supervisors:

prof. dr. ir. H.J. Broersma University of Twente

prof. dr. L. Wang Northwestern Polytechnical University

Members:

dr. W. Kern University of Twente

prof. dr. ir. A. Rensink University of Twente

prof. dr. X. Li Nankai University

prof. dr. ir. D. Paulusma Durham University

prof. dr. S. Zhang Northwestern Polytechnical University 



\section{Preface}

This thesis focuses on finding sufficient conditions for hamiltonian properties of graphs. The content contains the research results the author obtained when she studied as a PhD student both at Northwestern Polytechnical University (NPU) and the University of Twente (UT). After the introductory chapter (Chapter 1), the reader will find seven technical chapters, each of which has the structure of a journal paper.

In the introductory chapter, we introduce the general background and motivation for the research in this field, as well as an overview of our contributions. This chapter also contains some main research methods and some useful techniques. Most of the necessary terminology and notations that will be used in the subsequent chapters are also listed in this chapter. Some terms and notations that are used only in some specific situations are listed in the corresponding chapters.

Chapter 2 deals with sufficient conditions on the spectral radius and the signless Laplacian spectral radius for graphs to be hamiltonian. All of our results involve the characterization of the exceptional graphs, i.e., all the nonhamiltonian graphs that satisfy the condition.

In Chapters 3-5, the focus is on finding sufficient conditions for graphs to be Hamilton-connected. These conditions are based on the spectral radius, the signless Laplacian spectral radius, the Wiener index and the Harary index. These three chapters are based on research that was done while the author was working at NPU in Xi'an, China. The other chapters are based on research that was done at the UT. 
In Chapter 6 and Chapter 7, we investigate the hamiltonicity and Hamiltonconnectivity of $k$-connected graphs, respectively. We present some sufficient conditions on the spectral radius for $k$-connected graphs to have such hamiltonian properties.

In Chapter 8, we obtain a sufficient condition for a graph with a large minimum degree to be traceable and Hamilton-connected. This was inspired by the definition of a new concept and the results obtained in [49].

\section{Papers underlying this thesis}

[1] On sufficient spectral radius conditions for hamiltonicity (with H.J. Broersma, L.G. Wang and Y. Lu).

(Chapter 2)

[2] Sufficient conditions for Hamilton-connected graphs in terms of (signless Laplacian) spectral radius (with L.G. Wang and Y. Lu).

(Chapter 3)

[3] Signless Laplacian spectral conditions for Hamilton-connected graphs with large minimum degree (with L.G. Wang and Y. Lu).

(Chapter 4)

[4] Wiener index and Harary index on Hamilton-connected graphs with large minimum degree, Discrete Applied Mathematics 247 (2018) 180-185 (with L.G. Wang and Y. Lu).

(Chapter 5)

[5] On sufficient spectral radius conditions for hamiltonicity of $k$-connected graphs (with H.J. Broersma, L.G. Wang and Y. Lu).

(Chapter 6)

[6] On sufficient spectral radius conditions for Hamilton-connectivity of $k$ connected graphs (with H.J. Broersma, L.G. Wang and Y. Lu). (Chapter 7)

[7] Hamiltoncity of graphs with large minimum degree and without a large bipartite hole (with H.J. Broersma, L.G. Wang and Y. Lu).

(Chapter 8)

\section{Other papers by the author}

[1] Some sufficient spectral conditions on Hamilton-connected and traceable graphs, Linear and Multilinear Algebra, 65 (2017), 224-234 (with L.G. Wang). 
[2] Distance signless Laplacian spectral radius and Hamiltonian properties of graphs, Linear and Multilinear Algebra, 65 (2017), 2316-2323 (with L.G. Wang).

[3] Some sufficient conditions on k-connected graphs, Applied Mathematics and Computation, 325 (2018), 332-339 (with L.G. Wang and Y. Lu).

[4] Wiener-type invariants on graph properties, Filomat, 32 (2018), 489502 (with L.G. Wang and Y. Lu).

[5] Some sufficient conditions for some graph properties, Ars Combinatoria, accepted (with L.G. Wang and Y. Lu).

[6] Wiener-type invariants and Hamiltonian properties of graphs, submitted (with L.G. Wang and Y. Lu). 



\section{Contents}

Preface vii

1 Introduction 1

1.1 Terminology and notation $\ldots \ldots \ldots \ldots \ldots$

1.2 Degree conditions . . . . . . . . . . . . . . . . 9

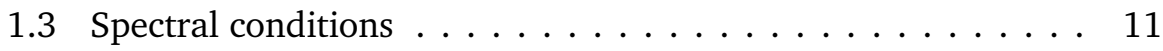

1.3.1 Spectral conditions for hamiltonian graphs . . . . . . 12

1.3.2 Spectral conditions for Hamilton-connected graphs . . . 14

1.4 Wiener index and Harary index conditions . . . . . . . 17

1.5 Some techniques and known results . . . . . . . . . . . 18

2 Sufficient spectral radius conditions for hamiltonicity 21

2.1 Introduction $\ldots \ldots \ldots \ldots \ldots \ldots \ldots \ldots \ldots \ldots \ldots$

2.1.1 Sufficient conditions in terms of the spectral radius . . 22

2.1.2 Sufficient conditions in terms of the signless Laplacian spectral radius . . . . . . . . . . . . . . . 24

2.2 Two useful lemmas . . . . . . . . . . . . . . . . 25

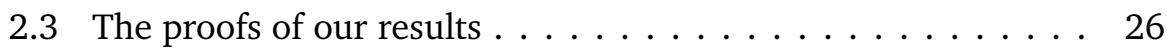

3 Sufficient conditions for Hamilton-connectivity 43

3.1 Introduction $\ldots \ldots \ldots \ldots \ldots \ldots \ldots \ldots \ldots$

3.2 A useful lemma $\ldots \ldots \ldots \ldots \ldots \ldots \ldots \ldots$

3.3 Proofs of the main results $\ldots \ldots \ldots \ldots \ldots \ldots \ldots$

4 Hamilton-connectivity of graphs with large minimum degree 65 
4.1 Introduction $\ldots \ldots \ldots \ldots \ldots \ldots \ldots \ldots \ldots$

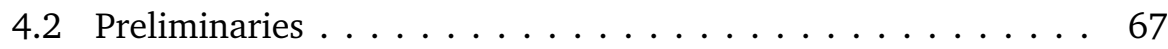

4.3 Main results and proofs $\ldots \ldots \ldots \ldots \ldots$

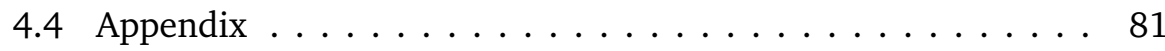

5 Wiener and Harary indices on Hamilton-connected graphs 85

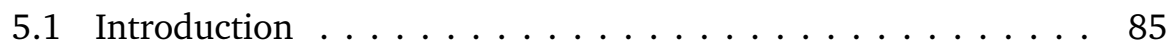

5.2 Wiener and Harary indices on $G$ for Hamilton-connectivity . . 89

5.3 Harary index on $\bar{G}$ for the Hamilton-connectivity . . . . . . . . 91

6 Hamiltonicity of $k$-connected graphs 95

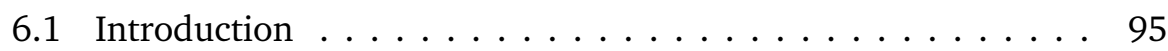

6.2 Two useful lemmas . . . . . . . . . . . . . . . . 98

6.3 Main results and proofs $\ldots \ldots \ldots \ldots \ldots . \ldots 9$

7 Hamilton-connectivity of $k$-connected graphs 113

7.1 Introduction $\ldots \ldots \ldots \ldots \ldots \ldots \ldots \ldots \ldots$

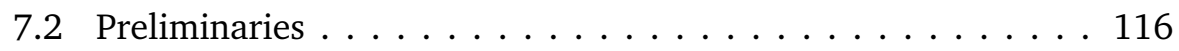

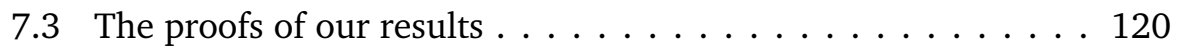

7.4 Appendix . . . . . . . . . . . . . . . . . . 128

8 Hamiltoncity of graphs without a large bipartite hole 131

8.1 Introduction . . . . . . . . . . . . . . . . . 131

8.2 Main results . . . . . . . . . . . . . . . . . . 133

8.3 The proofs . . . . . . . . . . . . . . . 134

$\begin{array}{ll}\text { Summary } & 141\end{array}$

Samenvatting (Summary in Dutch) 145

Bibliography 149

$\begin{array}{ll}\text { Acknowledgements } & 157\end{array}$

$\begin{array}{ll}\text { About the Author } & 161\end{array}$ 


\section{Chapter 1}

\section{Introduction}

Before defining the central concepts of this thesis formally in Section 1.1, let us start with a short informal introduction. A finite set of objects together with relationships between pairs of these objects can be (visually) represented by what we call a graph (or graph model) in mathematics. For example, given a map of a region or country, every city can be represented by a vertex (or by a small circle, i.e., a point on a piece of paper). Then an edge (or a line segment or curve on the piece of paper) joining two vertices (two points) can be used to indicate that the corresponding cities are connected by (direct) roads. Similarly, in a group of people one can use vertices (points) to denote all the persons of the group, and, if two persons know each other, one can draw an edge (line segment or curve) between the two corresponding vertices (points) to indicate this (binary) relationship. It should cost the reader little imagination to conclude from these two examples that graphs can represent many different types of objects together with their binary relationships. The mathematical field of graph theory mainly studies the structural properties of such graph models, and in many cases the aim is to find conditions on the graph that guarantee that certain wanted properties hold.

When facing a map or a bunch of people, several questions may be asked, e.g., "Is there a round trip that visits all the cities precisely once (except for the starting and terminating vertex)?", or "Can one arrange the seats 
around a table in such a way that all the people sitting there know the two people sitting next to them?". In fact, it is widely believed that graph theory originated from a similar practical problem, known as the problem of the Seven Bridges of Königsberg, proposed by Leonhard Euler in 1736. In that year, Euler wrote a paper in which he used concepts from graph theory to show that it was impossibility to pass all the seven bridges of Königsberg precisely once in a round trip.

As in the above examples, in general the graphs we consider in this thesis consist of a finite vertex set and a finite edge set, respectively representing a set of objects and the existing relationships between pairs of these objects. By using a graph, one can simplify a possibly complicated problem in ones daily life, by focussing on the essential information only. For answering the above question on the existence of a round trip visiting all the cities, the only relevant information is which pairs of cities are connected (directly) by roads. For arranging the seats, the only essential information is which pairs of persons know each other. Due to its wide applicability, and with the rapid development of computer science and the increase in computational capacity, graph theory has matured, and it has penetrated into many subject areas and plays an important role in scientific research work.

In a graph, a round trip which visits a subset of the vertices once, is called a cycle. If such a round trip passes through every vertex of the graph precisely once, we call it a Hamilton cycle. So, the two questions on the cities and the round table can both be translated into the question on the existence of a Hamilton cycle in a certain graph. The problem of determining whether a Hamilton cycle exists in a given graph has been proved to be NP-complete. Without going into details of what this means precisely, this implies that it is very unlikely that there exists an easily verifiable sufficient and necessary condition for the existence of a Hamilton cycle. This is the main reason why most researchers have focussed on establishing sufficient conditions, i.e., conditions on the graph that guarantee the existence of a Hamilton cycle.

In several chapters of this thesis, we focus on giving sufficient conditions for a graph to have a Hamilton cycle in terms of structural or algebraic parameters. Besides this, a large part of this thesis deals with sufficient conditions for other hamiltonian properties. The first of these other hamiltonian 
properties we consider is called traceability. It requires that there exists a Hamilton path in the graph, i.e., a path through the vertices and edges of the graph that visits all the vertices exactly once (and does not return to the starting vertex). We say a graph is traceable if it contains a Hamilton path, and traceable from a vertex $x$ if it contains a Hamilton $x$-path, i.e., a Hamilton path starting at vertex $x$. So, the second other hamiltonian property we deal with is that the graph is traceable from every arbitrary vertex. The last hamiltonian property we take into account is Hamilton-connectivity, which requires that any two distinct vertices can be connected by a Hamilton path. During the past decades, people have focused on finding sufficient or necessary conditions for hamiltonian properties from many different angles, using graph concepts such as the degree of the vertices, forbidden subgraphs, the Wiener index, the spectral radius, and so on. The results in this thesis mainly involve the following three types of conditions. We recall here that formal definitions of the concepts we mention below as well as references to related work appear in Section 1.1.

\section{Degree conditions}

Intuitively, it is more likely for a graph to have a Hamilton cycle if every vertex has a large degree, i.e., has many neighbors, i.e., vertices that are joined to it by an edge. The degree of a vertex is defined as the cardinality of its neighbor set. In fact, Dirac proved back in the 1950s that if every vertex has degree at least half of the number of vertices of the graph, then this graph contains a Hamilton cycle. This result of Dirac motivated much of the research in this field. In Chapter 8, we present some sufficient conditions for traceability and Hamilton-connectivity of graphs with a large minimum degree, but degree considerations play a central role throughout the whole thesis.

\section{Spectral conditions}

Spectral graph theory plays a significant role in modern graph theory and is an important research area of algebraic graph theory. It mainly uses concepts of linear algebra, especially the theory of matrices, to study graph theory and 
its applications. For a given graph, there exists a variety of associated matrices, such as the adjacency matrix, Laplacian matrix, the signless Laplacian matrix, distance matrix, and so on, to be defined later in the thesis. Spectral graph theory mainly focusses on spectral properties of these algebraic representations, i.e., properties of the eigenvalues of these matrices and their relationship with structural properties of the graphs.

The spectrum of a graph is the (multi)set of eigenvalues of (one of) these matrices. In particular, the spectrum of a graph can reflect much significant information of graph invariants or properties. One of the important fields of spectral graph theory is to study the relationship between the spectra of graphs and the structure of graphs.

In the past few years, the following problem has been extensively studied by many graph theory researchers.

Problem 1.1. For a given graph $F$, what is the maximum spectral radius of a graph $G$ on $n$ vertices that does not contain a subgraph isomorphic to $F$ ?

In case $F$ is a Hamilton cycle, the above problem can be considered as using the spectral radius to give a sufficient condition for a graph to have a Hamilton cycle. In 2010, Fiedler and Nikiforov obtained some bounds on the spectral radius that imply the existence of Hamilton cycles (or paths) in a graph. This inspired other graph theorists to perform further research on this problem. Most parts of this thesis focus on finding sufficient spectral conditions for hamiltonian properties of graphs.

In Chapter 2, we consider the general case of graphs with minimum degree at least $k$. There we present some new results on sufficient conditions for the hamiltonicity of graphs in terms of their spectral radius and their signless Laplacian spectral radius, thereby extending some previously known results. In Chapters 3 and 4, we focus on Hamilton-connectivity instead of hamiltonicity. In Chapter 3, we consider the special case when the minimum degree of the graph is at least 3. In Chapter 4, we mainly deal with graphs with minimum degree at least $k$. In these two chapters, we prove results on spectral radius conditions and signless Laplacian spectral radius conditions that imply Hamilton-connectedness. In Chapters 6 and 7, we continue to study spectral conditions for hamiltonicity and Hamilton-connectivity of 
graphs. Different from the former chapters, in these two chapters we focus on $k$-connected graphs. All our results involve the characterization of exceptional graphs, i.e., all the nonhamiltonian or non-Hamilton-connected graphs that satisfy the imposed conditions.

\section{Wiener index and Harary index conditions}

Over the past decades, topological indices have attracted much attention from scholars. These indices are from the field of chemical graph theory. A topological index is regarded as a molecular descriptor to be calculated based on the molecular graph of a chemical compound. Indeed, many topological indices have been used to characterize the topology of a graph, and they are widely known as graph invariants.

One of the most studied topological indices is the Wiener index. It was introduced in 1947 by Harry Wiener, who used it for modeling the shape of organic molecules. This index, which is based on the information in the distance matrix of a graph, is an important concept in graph theory. The other topological index we have used in this thesis is called the Harary index, which is the reciprocal version of the Wiener index. It was introduced independently by Ivanciuc et al. and Plavšić et al. in 1993 for the characterization of molecular graphs. It has been named in honor of Professor Frank Harary on the occasion of his 70th birthday.

Since the Wiener index and the Harary index are concise and useful for characterizing the structure of a graph, many graph theorists began to use them for establishing sufficient conditions for graphs possessing hamiltonian properties. In Chapter 5, we use these two topological indices to study the Hamilton-connectivity of graphs with minimum degree at least $k$. We present two sufficient conditions in terms of the Wiener index and the Harary index, and we also characterize the extremal graphs.

In the next section, we will give some basic definitions of concepts which are frequently used throughout this thesis. 


\subsection{Terminology and notation}

Throughout this thesis, we only consider simple graphs without loops and multiple edges. For terminology and notation not defined but used here, we refer the reader to the textbook of Bondy and Murty [6].

Let $G=(V(G), E(G))$ be a graph with vertex set $V(G)$ and edge set $E(G)$. We use $n(G)=|V(G)|$ and $e(G)=|E(G)|$ to denote two basic parameters that are called the order and size of $G$, respectively. The complement $\bar{G}$ of $G$ is the graph on $V(G)$ with edge set $[V(G)]^{2} \backslash E(G)$ (all the pairs that are not joined by an edge in $G$ are joined by an edge in $\bar{G}$, and vice versa). If two vertices $u, v \in V(G)$ are joined by an edge $e \in E(G)$, then we say $u$ and $v$ are adjacent, and we say that $e$ is incident with $u$ (and $v$ ). Two distinct adjacent vertices are also called neighbors. For two vertex subsets $X$ and $Y$, we say $X$ is adjacent to $Y$ if every vertex in $X$ is adjacent to every vertex in $Y$. Let $E(X, Y)$ denote the set of edges with one end in $X$ and one in $Y$. A complete graph is a graph in which any two distinct vertices are adjacent. We use $K_{n}$ to denote the complete graph of order $n$. We use $G=G[X, Y]$ to denote a bipartite graph the vertex set of which can be partitioned into two nonempty subsets $X$ and $Y$ such that every edge has one end in $X$ and one end in $Y$.

For $v \in V(G)$, the set of vertices adjacent to $v$ in $G$ is denoted by $N_{G}(v)$. We call $d_{G}(v)=\left|N_{G}(v)\right|$ the degree of $v$. Moreover, we define $N_{G}[v]=$ $N_{G}(v) \cup\{v\}$, and we use $\delta(G)$ to denote the minimum degree of (the vertices of) $G$. If the graph $G$ is clear from the context, we will drop the subscript $G$ from the above notations. A graph $F$ is called a subgraph of $G$ if $V(F) \subseteq V(G)$ and $E(F) \subseteq E(G)$. For $v \in V(G)$ and two subgraphs $H$ and $R$ of $G$, denote by $N_{H}(v)=\{u \in V(H) \mid u v \in E(G)\}$ and $N_{H}(R)=\left(\bigcup_{u \in V(R)} N_{H}(u)\right) \backslash V(R)$ the neighbors of the vertex $v$ and the subgraph $R$ in $H$, respectively. The independence number $\alpha(G)$ of $G$ is the cardinality of the largest independent (mutually nonadjacent) set of vertices.

For two vertex-disjoint graphs $G$ and $H$, the (disjoint) union $G+H$ is the graph with vertex $V(G) \cup V(H)$ and edge set $E(G) \cup E(H)$. The join $G \vee H$ is the graph obtained from the disjoint union of $G$ and $H$ by adding edges joining every vertex of $G$ to every vertex of $H$. The union of $k$ vertex-disjoint copies of the same graph $G$ is denoted by $k G$. If $S$ is a nonempty subset 
of $V(G)$, then $G[S]$ denotes the subgraph of $G$ induced by $S$, i.e., the graph with vertex set $S$ containing all edges of $G$ that join two vertices of $S$. If an induced subgraph is complete, then we call it a clique. The clique number of a graph $G$, denoted by $\omega(G)$, is the number of vertices in a largest clique of $G$. A spanning subgraph of a graph $G$ is a subgraph obtained by edge deletions only (a subgraph of $G$ on the same vertex set $V(G)$ ). If $H$ is a spanning subgraph of $G$, we use $G-H$ to denote the graph with vertex set $V(G)$ and edge set $E(G) \backslash E(H)$. For two vertex-disjoint nonempty subsets $S$ and $T$ of $V(G), G[S, T]$ denotes the bipartite graph on vertex set $S \cup T$ with all edges of $G$ that join a vertex of $S$ and a vertex of $T$.

A path in $G$ is a linear sequence of distinct vertices of $G$ such that any two consecutive vertices in the sequence are adjacent in $G$, i.e., a sequence of distinct vertices $v_{1}, v_{2}, \ldots, v_{k}$ such that $v_{i} v_{i+1} \in E(G)$ for all $i \in\{1,2, \ldots, k-1\}$. Likewise, a cycle on three or more vertices is a cyclic sequence so that any two consecutive vertices in the sequence are adjacent in $G$, i.e., a sequence of distinct vertices $v_{1}, v_{2}, \ldots, v_{k}, v_{1}$ such that $v_{i} v_{i+1} \in E(G)$ for all $i \in\{1,2, \ldots, k-$ $1\}$ and additionally $v_{k} v_{1} \in E(G)$. The length of a path or a cycle is the number of its edges. We denote by $P_{n}$ a path of order $n$ and by $C_{n}$ a cycle with $n$ vertices.

As we mentioned in the beginning of this introductory chapter, a Hamilton cycle is a cycle passing through (containing) all the vertices of the graph. Similarly, a Hamilton path is a path that contains every vertex of the graph. A graph is called hamiltonian if it contains a Hamilton cycle, and it is called traceable if it contains a Hamilton path. A Hamilton-connected graph is a graph in which any two distinct vertices are connected by a Hamilton path. We say a graph is traceable from $x$ if it contains a Hamilton $x$-path, i.e., a Hamilton path starting from the vertex $x$. Obviously, a hamiltonian graph is also traceable, and a Hamilton-connected graph is also hamiltonian, but the reverse statements are not true in general.

Next we turn to the connectivity of a graph. A graph is connected if for every partition of its vertex set into two nonempty sets $X$ and $Y$, there is an edge with one end in $X$ and one end in $Y$. Alternatively, a graph is connected if there exists a path between any two distinct vertices of the graph; otherwise the graph is called disconnected. A connected graph $G$ is said to 
be $k$-connected (or $k$-vertex connected) if it has more than $k$ vertices and remains connected whenever fewer than $k$ vertices (together with their incident edges) are removed from $G$. The connectivity $\kappa(G)$ of $G$ is the maximum value of $k$ for which $G$ is $k$-connected.

We continue with some algebraic definitions that are mostly used in this thesis. Let $G$ be a graph with vertex set $\left\{v_{1}, v_{2}, \ldots, v_{n}\right\}$. Then the adjacency matrix $A(G)$ of $G$ is the symmetric $n \times n$-matrix with entries $A(i, j)=1$ if and only if $v_{i} v_{j} \in E(G)$ and zeros elsewhere. The diagonal degree matrix $D(G)$ of $G$ is the $n \times n$-matrix with entries $D(i, i)=d\left(v_{i}\right)$ and zeros elsewhere. The matrix $Q(G)=D(G)+A(G)$ is known as the signless Laplacian matrix of $G$. The largest eigenvalue of $A(G)$, denoted by $\rho(A(G))$ or $\rho(G)$, is called the spectral radius of $G$. The largest eigenvalue of $Q(G)$, denoted by $q(Q(G))$ or $q(G)$, is called the signless Laplacian spectral radius of $G$.

For two vertices $u$ and $v$, the distance $d_{G}(u, v)$ between $u$ and $v$ in $G$ is the length of a shortest path connecting $u$ and $v$ in $G$. Denote by $\operatorname{diam}(G)$ the diameter of $G$, i.e., the largest distance among all pairs of vertices of $G$. The Wiener index, denoted by $W(G)$, was introduced in 1947 by Wiener [60]. It is defined as

$$
W(G)=\sum_{\{u, v\} \subseteq V(G)} d_{G}(u, v) .
$$

The Harary index, denoted by $H(G)$, has been introduced by Ivanciuc et al. [35] and Plavšić et al. [56]. It is defined as

$$
H(G)=\sum_{\{u, v\} \subseteq V(G)} \frac{1}{d_{G}(u, v)} .
$$

Note that in any disconnected graph $G$, the distance is infinite for any two vertices from distinct components. Therefore its reciprocal is equal to 0 . Thus, we can validly define the Harary index of a disconnected graph $G$ as follows:

$$
H(G)=\sum_{i=1}^{k} H\left(G_{i}\right),
$$

where $G_{1}, G_{2}, \ldots, G_{k}$ are the components of $G$.

Apart from the above more or less traditional and standard definitions 
from the perspective of structure or algebra of a graph, next we focus on a relatively new graph parameter, named the bipartite-hole-number, which was introduced by McDiarmid and Yolov [49].

Definition 1.1 (McDiarmid and Yolov [49]). An $(s, t)$-bipartite-hole in a graph $G$ consists of two disjoint sets of vertices $S$ and $T$ with $|S|=s$ and $|T|=t$ such that $E(S, T)=\emptyset$. We define the bipartite-hole-number $\widetilde{\alpha}(G)$ to be the least integer $r$ that may be written as $r=s+t-1$ for some positive integers $s$ and $t$ such that $G$ does not contain an $(s, t)$-bipartite-hole.

As stated in [49], an equivalent definition of $\widetilde{\alpha}(G)$ is the maximum integer $r$ such that $G$ contains an $(s, t)$-bipartite-hole for every pair of nonnegative integers $s$ and $t$ with $s+t=r$.

For convenience of the reader in understanding some of our proofs, we also add the next notation here. Let $C$ be a cycle in $G$. Then we use $\vec{C}$ to denote the cycle $C$ with a given fixed cyclic orientation (so, one of the two possible choices for traversing all the vertices of $C$ in a cyclic way). For $u, v \in C$, we use $\vec{C}[u, v]$ to denote the consecutive vertices on $C$ from $u$ to $v$ in the direction specified by $\vec{C}$. The same vertices, in the reverse order, are given by $\overleftarrow{C}[v, u]$. Both $\vec{C}[u, v]$ and $\overleftarrow{C}[v, u]$ are considered as paths as well as vertex sets. We use similar notations for paths with a given orientation.

Other notations and definitions that are not included here will appear at the first occurrence where they are used in the thesis.

In the next three sections, we focus on degree conditions, spectral conditions, and Wiener index and Harary index conditions for hamiltonicity or Hamilton-connectivity of graphs. We will also introduce the main results of this thesis, as well as some background, motivation and related results.

\subsection{Degree conditions}

Degree conditions are the earliest proposed conditions that deal with path and cycle properties. Intuitively, increasing the degree of the vertices of a graph will increase the probability for the graph to have a Hamilton cycle. This observation is the basis for many results. Up to now, degree conditions 
are known as the classic approach to hamiltonian problems and still the most studied conditions. The basis for subsequent research in this field is the following result given by Dirac in 1952.

Theorem 1.1 (Dirac [16]). A graph $G$ with $n \geq 3$ vertices is hamiltonian if $\delta(G) \geq n / 2$.

There are many generalizations and extensions of Dirac's Theorem. The following theorem is due to Ore.

Theorem 1.2 (Ore [55]). If $G$ is a graph on $n \geq 3$ vertices such that $d(u)+$ $d(v) \geq n$ for every pair $u, v$ of nonadjacent vertices, then $G$ contains a Hamilton cycle.

Obviously, Ore's Theorem implies Dirac's Theorem, and can in fact be shown to be more generally applicable. Both of these two results are best possible, in the sense that the minimum degree or minimum degree sum in the above two theorems can not be lowered without affecting the conclusion.

Bondy and Chvátal further generalized Ore's Theorem. We refer the interested reader to the book [6] for more details. Furthermore, the condition in Ore's Theorem was generalized by Fan [20], who proved that a 2-connected graph $G$ of order $n$ is hamiltonian if $\max (d(u), d(v)) \geq n / 2$ for every pair of nonadjacent vertices $u, v$ with distance 2 (See, e.g., the survey [15]).

Over the years, Ore's Theorem and Dirac's Theorem inspired many graph theorists to propose other sufficient conditions for hamiltonian properties in terms of the degrees and neighborhoods of vertices of a graph, such as the minimum degree sum over all independent sets on $t$ vertices, the minimum cardinality of the neighborhood union over all independent sets on $t$ vertices of a graph, the minimum implicit degree, and so on. We omit the details here, because the research reported in this thesis bears no close relationship to the above areas. There are several survey papers on hamiltonian graph theory in which the interested reader can find more details on the above concepts and results (See [4, 7, 22, 28-30,36, 38]).

Given two disjoint sets of vertices of a graph, if there is no edge between them, we call it a bipartite hole. It is natural to ask if such a hole is necessary to construct a nonhamiltonian graph with a large minimum degree. 
McDiarmid and Yolov proved that this is indeed the case. In [49], the authors present a tight extremal threshold for the existence of Hamilton cycles in graphs with a large minimum degree and without a large bipartite hole. For details of their results, we refer the reader to Chapter 8 .

Similarly as for the analogues of Dirac's Theorem for traceable graphs and Hamilton-connected graphs, the question comes to mind if it is possible to construct a nontraceable or non-Hamilton-connected graph with a large minimum degree by using the bipartite hole number. In fact, the analogous sufficient condition for a graph admitting a Hamilton path can be easily deduced from the result of McDiarmid and Yolov. In Chapter 8, we give the specific statement and its proof. By using this result, we also provide a sufficient condition for the existence of many edge-disjoint Hamilton paths.

Furthermore, we also obtain a sufficient condition for a graph with a large minimum degree to be Hamilton-connected. Our result generalizes the analogue of Dirac's Theorem for Hamilton-connected graphs stating that a graph $G$ of order $n$ is Hamilton-connected if $\delta(G) \geq \frac{n+1}{2}$.

\subsection{Spectral conditions}

The most important research fields of spectral graph theory generally include: using the spectra of graphs to determine the structure of graphs; finding cospectral graphs (i.e., nonisomorphic graphs that have the same graph spectrum); estimates, lower and upper bounds for the eigenvalues of graphs; relationships between the spectra of graphs and other invariants of graphs, such as graph energy (i.e., the sum of the absolute values of the eigenvalues of the adjacency matrix of the graph), chromatic number (i.e., the smallest number of colors needed to color the vertices of the graph such that no two adjacent vertices share the same color), and so on.

Extremal graph theory is also an important branch of graph theory. In extremal graph theory, people study the relationship between various graph invariants, such as the order, size, girth (i.e., the length of a shortest cycle contained in the graph), connectivity, minimum degree, and so on, and also 
use these invariants to study extremal (maximal or minimal) graphs which satisfy a certain property.

In recent years, a popular trend in extremal graph theory is to study extremal properties of graphs by means of eigenvalues of various matrices, within the area of graph theory that is usually called spectral extremal graph theory.

Spectral extremal graph theory mainly study the relationships between graph properties and the eigenvalues of certain matrices associated with the graph. As Nikiforov stated in [51], much of classical extremal graph theory has been translated into spectral statements, and this translation has also brought enhancement. We refer to the survey [51] for details.

In [9], Brualdi and Solheid raised the following spectral problem.

Problem 1.2. What is the maximum spectral radius of a graph $G$ on $n$ vertices belonging to a specified class of graphs?

If we combine this problem with Turán's problem, then we obtain Problem 1.1 which is mentioned in the early part of this chapter. In this thesis, we mainly study the case when $F$ is a Hamilton cycle (or path) in Problem 1.1, i.e., the relationship between the spectral radius and hamiltonian properties of a graph.

\subsubsection{Spectral conditions for hamiltonian graphs}

Let $A(G)$ be the adjacency matrix of the graph $G$. Denote by $\rho(G)$ the spectral radius of $G$, i.e., the largest eigenvalue of $A(G)$. Recall that we use $q(G)$ to denote the signless Laplacian spectral radius of $G$, i.e., the largest eigenvalue of $Q(G)$.

We let $K_{n-1}+v$ denote the graph that consists of a $K_{n-1}$ together with an isolated vertex. In 2010, Fiedler and Nikiforov [25] presented the following sufficient condition for hamiltonicity of graphs.

Theorem 1.3 (Fiedler and Nikiforov [25]). Let $G$ be a graph of order $n$.

(i) If $\rho(G) \geq n-2$, then $G$ contains a Hamilton path unless $G=K_{n-1}+v$. 
(ii) If $\rho(G)>n-2$, then $G$ contains a Hamilton cycle unless $G=K_{1} \vee\left(K_{n-2}+\right.$ $\left.K_{1}\right)$.

In the same paper, the authors also gave a subtler condition for hamiltonicity involving the spectral radius of the complement of the graph.

Theorem 1.4 (Fiedler and Nikiforov [25]). Let $G$ be a graph of order $n$, and let $\rho(\bar{G})$ be the spectral radius of its complement.

(i) If $\rho(\bar{G}) \leq \sqrt{n-1}$, then $G$ contains a Hamilton path unless $G=K_{n-1}+v$.

(ii) If $\rho(\bar{G}) \leq \sqrt{n-2}$, then $G$ contains a Hamilton cycle unless $G=K_{1} \vee$ $\left(K_{n-2}+K_{1}\right)$.

Since $G=K_{n-1}+v$ is disconnected, obviously it is not traceable. And note that the graph $G=K_{1} \vee\left(K_{n-2}+K_{1}\right)$ is clearly not hamiltonian: it has a vertex with degree 1 . This also implies that deleting the vertex $v$ corresponding to the first $K_{1}$, the graph $G-v$ has two components, so $G$ is not 1-tough. In general, a graph $G$ is called 1-tough if the number of components of $G-S$ is at most $|S|$ for every cut set $S$ of $G$. Being 1-tough is an obvious necessary condition for being hamiltonian (See [6] for details).

The work of [25] spurred the interest of several research groups. Zhou [66] presented tight conditions on the signless Laplacian spectral radius of a graph for the existence of Hamilton paths and cycles.

Theorem 1.5 (Zhou [66]). Let $G$ be a graph on $n$ vertices with complement $\bar{G}$.

(i) If $q(\bar{G}) \leq n$ and $G \notin \mathbb{E} \mathbb{P}_{n}$, then $G$ contains a Hamilton path.

(ii) If $n \geq 3, q(\bar{G}) \leq n-1$ and $G \notin \mathbb{E} \mathbb{C}_{n}$, then $G$ contains a Hamilton cycle.

In Theorem 1.5, $\mathbb{E} \mathbb{P}_{n}$ denotes the set of graphs consisting of the following three types of graphs on $n$ vertices: (a) a regular graph of degree $\frac{n}{2}-1$, (b) a graph consisting of two complete components, and (c) the join of a regular graph of degree $\frac{n}{2}-1-r$ and a graph on $r$ vertices, where $1 \leq r \leq \frac{n}{2}-1$. $\mathbb{E C}_{n}$ denotes the set of graphs consisting of the following two types of graphs on $n$ vertices: (a) the join of a trivial graph $\left(K_{1}\right)$ and a graph consisting of two 
complete components, and (b) the join of a regular graph of degree $\frac{n-1}{2}-r$ and a graph on $r$ vertices, where $1 \leq r \leq \frac{n-1}{2}$.

In a more recent paper, Lu et al. [47] focussed on bipartite graphs. Let $G:=G[X, Y]$ be a bipartite graph. The quasi-complement of $G$, denoted by $G^{*}$, is the graph obtained from $G$ as follows: $V\left(G^{*}\right)=V(G)$ and for $x \in X$, $y \in Y, x y \in E\left(G^{*}\right)$ if and only if $x y \notin E(G)$. In [47], the authors gave two sufficient conditions for a bipartite graph to be hamiltonian and for a (not necessarily bipartite) graph to be traceable, respectively.

Theorem 1.6 (Lu, Liu and Tian [47]). Let $G:=G[X, Y]$ be a bipartite graph and $G^{*}$ the quasi-complement of $G$, where $|X|=|Y|=n \geq 2$. Let $\rho\left(G^{*}\right)$ be the spectral radius of $G^{*}$. If $\rho\left(G^{*}\right) \leq \sqrt{n-1}$, then $G$ is hamiltonian unless $G=K_{n, n-1}+e$.

Here, let $K_{n, n-1}=(X, Y)$ be a complete bipartite graph with $|X|=n$ and $|Y|=n-1$. Then $K_{n, n-1}+e$ is the graph obtained from $K_{n, n-1}$ by adding a pendent edge to one of the vertices in $X$ (adding a new vertex and joining it to precisely one vertex of $X$ by an edge).

Theorem 1.7 (Lu, Liu and Tian [47]). Let $G$ be a connected graph of order $n \geq 5$. If $\rho(G) \geq \sqrt{(n-3)^{2}+2}$, then $G$ is traceable.

For more results on spectral radius and hamiltonicity of graphs, we refer to $[2,10,26,40-42,46,52,53,69,70]$. We will give the details of some of these results in Chapter 2 .

Looking at existing results, one way to extend such results is by relaxing the bounds in the respective conditions, and then trying to characterize the exceptional graphs.

In Chapter 2, we focus on graphs with minimum degree $\delta(G) \geq k$. Our aim is to extend some previous results on spectral radius conditions for hamiltonicity of graphs. We refer to Chapter 2 for more background and related results, and for our main theorems and their proofs.

\subsubsection{Spectral conditions for Hamilton-connected graphs}

Besides finding sufficient conditions for hamiltonicity of graphs, we also focus on related sufficient conditions for graph properties that are stronger than the 
property of having a Hamilton cycle. In this case we consider the Hamiltonconnectedness of graphs.

The following sufficient condition involving the number of edges is due to Ore [54].

Theorem 1.8. (Ore [54]) Let $G$ be a graph on $n$ vertices. If

$$
e(G) \geq\left(\begin{array}{c}
n-1 \\
2
\end{array}\right)+3
$$

then $G$ is Hamilton-connected.

Observing that $\delta \geq 3$ is a trivial necessary condition for $G$ to be Hamiltonconnected, Zhou (the author of this thesis) and Wang [70] refined the above edge number condition, as follows.

Theorem 1.9. (Zhou and Wang [70]) Let $G$ be a connected graph on $n \geq 6$ vertices with minimum degree $\delta \geq 3$. If

$$
e(G) \geq\left(\begin{array}{c}
n-2 \\
2
\end{array}\right)+6
$$

then $G$ is Hamilton-connected unless $G \in \mathbb{N P}_{1}=\left\{K_{3} \vee\left(K_{n-5}+2 K_{1}\right), K_{6} \vee\right.$ $6 K_{1}, K_{4} \vee\left(K_{2}+3 K_{1}\right), 5 K_{1} \vee K_{5}, K_{4} \vee\left(K_{1,4}+K_{1}\right), K_{4} \vee\left(K_{1,3}+K_{2}\right), K_{3} \vee K_{2,5}, K_{4} \vee$ $\left.4 K_{1}, K_{3} \vee\left(K_{1}+K_{1,3}\right), K_{3} \vee\left(K_{1,2}+K_{2}\right), K_{2} \vee K_{2,4}\right\}$.

For spectral conditions, in [64], Yu and Fan have established sufficient conditions for a graph to be Hamilton-connected in terms of the spectral radius and the signless Laplacian spectral radius. We use $G=K_{n-1}+e+e^{\prime}$ to denote the graph consisting of a $K_{n-1}$ together with a new vertex that is joined to precisely two vertices of the $K_{n-1}$ by the edges $e$ and $e^{\prime}$, respectively.

Theorem 1.10. (Yu and Fan [64]) Let $G$ be a graph on $n$ vertices.

(i) If $\rho(G)>-\frac{1}{2}+\sqrt{n^{2}-3 n+\frac{17}{4}}$, then $G$ is Hamilton-connected unless $G=K_{n-1}+e+e^{\prime}$.

(ii) If $\rho(\bar{G})<\sqrt{\frac{(n-2)^{2}}{n}}$, then $G$ is Hamilton-connected. 
(iii) If $q(G)>2 n-4+\frac{2}{n-1}$, then $G$ is Hamilton-connected unless $G=K_{n-1}+$ $e+e^{\prime}$.

Recently, Zhou and Wang [70] obtained the following sufficient conditions on the spectral radius and the signless Laplacian spectral radius for a graph to be Hamilton-connected, thereby extending the result of $\mathrm{Yu}$ and Fan [64], in the following sense.

Theorem 1.11. (Zhou and Wang [70]) Let $G$ be a connected graph on $n \geq 6$ vertices with minimum degree $\delta \geq 3$.

(i) If $\rho(G) \geq \sqrt{n^{2}-6 n+19}$, then $G$ is Hamilton-connected.

(ii) If $q(G) \geq 2 n-6+\frac{14}{n-1}$, then $G$ is Hamilton-connected unless $G=K_{4} \vee 4 K_{1}$.

In Chapter 3, we first examine a condition involving a better lower bound than in Theorem 1.9 and characterize all exceptional graphs. Then we present two sufficient conditions in terms of the spectral radius and the signless Laplacian spectral radius for a graph to be Hamilton-connected, thereby extending Theorem 1.11. From these two results, we also obtain two corollaries, in which the extremal graphs with maximum spectral radius and maximum signless Laplacian spectral radius among all non-Hamilton-connected graphs are determined.

Recently, by involving the minimum degree of the graph as a new parameter, many graph theorists tried to obtain analogues of known results for graphs with minimum degree $\delta(G) \geq k$. In Chapter 4, we follow this thread and establish a signless Laplacian analogue of a result due to Nikiforov [52] for Hamilton-connected graphs with large minimum degree, thereby generalizing Theorem 1.11. For more details, we refer to Chapter 4.

In Chapters 6 and 7, we continue to study the relationships between the spectral radius and hamiltonian properties of graphs. Different from the former chapters, here we mainly deal with $k$-connected graphs. In Chapter 6 , we present new sufficient conditions based on the spectral radius for the hamiltonicity and traceability of $k$-connected graphs, thereby strengthening some previous results on graphs with minimum degree $\delta(G) \geq k$. In Chapter 7, we present sufficient conditions for a $k$-connected graph to be 
Hamilton-connected in terms of the spectral radius, again extending the analogues for graphs with minimum degree $\delta(G) \geq k$.

\subsection{Wiener index and Harary index conditions}

As we introduced before, the Wiener index and the Harary index are two popular and concise topological indices. The distance matrix of a graph and its spectral properties have been widely studied. Since these two indices are based on the distances of pairs of vertices of graphs, and since finding sufficient conditions for graphs possessing certain properties is a hot trend in graph theory, over the years several graph theorists considered using the Wiener index and the Harary index to give sufficient conditions for hamiltonian properties of a graph.

In 2013, Hua and Wang [34] gave a sufficient condition for a graph to be traceable in terms of the Harary index.

Theorem 1.12. (Hua and Wang [34]) Let $G$ be a graph of order $n \geq 4$. If

$$
H(G) \geq \frac{1}{2} n^{2}-\frac{3}{2} n+\frac{5}{2},
$$

then $G$ is traceable unless $G \in\left\{K_{1} \vee\left(K_{n-3}+2 K_{1}\right), K_{2} \vee\left(K_{2}+3 K_{1}\right), K_{4} \vee 6 K_{1}\right\}$.

Yang [63] gave a sufficient condition based on the Wiener index for a graph to be traceable.

Theorem 1.13. (Yang [63]) Let $G$ be a graph of order $n \geq 4$. If

$$
W(G) \leq \frac{(n+5)(n-2)}{2}
$$

then $G$ is traceable unless $G \in\left\{K_{1} \vee\left(K_{n-3}+2 K_{1}\right), K_{2} \vee\left(K_{2}+3 K_{1}\right), K_{4} \vee 6 K_{1}\right\}$.

Unfortunately, there were some errors in the proofs of these two papers. In $[44,45]$, Liu et al. corrected their results, respectively, and also proposed some sufficient conditions for hamiltonian graphs based on the Wiener index and the Harary index. By imposing an additional minimum degree condition, 
Hua et al. [33] obtained sufficient conditions for hamiltonicity and traceability of connected graphs and of connected balanced bipartite graphs in terms of the Wiener index and the Harary index. Here we omitted the details but we have put them in Chapter 5 .

We continued to study the relationships between the Wiener and the Harary indices and hamiltonian properties of graphs. We focussed on graphs with minimum degree $\delta(G) \geq k$. In Chapter 5 , we give sufficient conditions for connected graphs to be Hamilton-connected and traceable from every vertex in terms of the Wiener index and the Harary index. Our results also characterize the non-Hamilton-connected graphs and not traceable from every vertex graphs with minimum Wiener index and maximum Harary index, respectively.

In the final section of this introductory chapter, we will introduce some techniques, and state some known results that we frequently use in our proofs as lemmas.

\subsection{Some techniques and known results}

We start this section by explaining the concept of the Kelmans transformation, a very useful tool in the context of graph radii.

\section{Kelmans transformation}

Given a graph $G$ and two specified vertices $u$ and $v$, construct a new graph $G^{*}$ by replacing all edges $v x$ by $u x$ for $x \in N_{G}(v) \backslash N_{G}[u]$. Obviously, the new graph $G^{*}$ has the same number of vertices and edges as $G$, and all vertices different from $u$ and $v$ retain their degrees. The vertices $u$ and $v$ are adjacent in $G^{*}$ if and only if they are adjacent in $G$. This construction was first introduced in [37], and is nowadays known as the Kelmans transformation.

The relevance of the Kelmans transformation in the context of radii is expressed by the following two lemmas.

Lemma 1.14 (Csikvári [13]). Let $G$ be a graph, and let $G^{*}$ be a graph obtained from $G$ by some Kelmans transformation. Then $\rho(G) \leq \rho\left(G^{*}\right)$. 
Lemma 1.15 (Li and Ning [41]). Let $G$ be a graph, and let $G^{*}$ be a graph obtained from $G$ by a Kelmans transformation. Then $q(G) \leq q\left(G^{*}\right)$.

We now turn to the useful concept of equitable partitions.

\section{Equitable partitions}

Suppose $M$ is a symmetric real $n \times n$-matrix whose rows and columns are indexed by $X=\{1, \ldots, n\}$. Let $\pi=\left\{X_{1}, \ldots, X_{m}\right\}$ be a partition of $X$. Let $M$ be partitioned according to $\left\{X_{1}, \ldots, X_{m}\right\}$, i.e.,

$$
M=\left(\begin{array}{ccc}
M_{11} & \ldots & M_{1 m} \\
\vdots & & \vdots \\
M_{m 1} & \ldots & M_{m m}
\end{array}\right),
$$

where $M_{i j}$ denotes the block of $M$ formed by the rows in $X_{i}$ and the columns in $X_{j}$. Let $b_{i j}=\frac{\mathbf{1}^{T} M_{i j} \mathbf{1}}{\left|X_{i}\right|}$, i.e., the average row sum of $M_{i j}$, where 1 is the column vector (of the correct dimension) with all entries equal to 1 . Then the matrix $M / \pi=\left(b_{i j}\right)_{m \times m}$ is called the quotient matrix of $M$. If the row sum of each block $M_{i j}$ is a constant, then the partition is called equitable.

If $M$ denotes the adjacency matrix $A(G)$ (or signless Laplacian matrix $Q(G)$ ) of a graph $G$, and $M$ admits an equitable partition $\left\{X_{1}, \ldots, X_{m}\right\}$, then the corresponding partition of $V(G)$ is called an equitable partition of $G$. This partition has the property that for every vertex $v \in X_{i}$, the number of neighbors of $v$ in $X_{j}$ is the same, for all $i, j \in\{1,2, \ldots, m\}$. Any partition with the latter property is clearly also an equitable partition of $G$.

The next result shows in which sense the use of equitable partitions can facilitate determining the radii of a graph $G$.

Lemma 1.16 (Godsil and Royle [27]). Let $G$ be a graph, and let $\pi$ be an equitable partition of $G$. Then $\rho(G)=\rho(A(G))=\rho(A(G) / \pi)$ and $q(G)=$ $q(Q(G))=q(Q(G) / \pi)$.

\section{Some inequalities for the spectral radius}


We complete this section and chapter by some known inequalities that we will use throughout the thesis. The first result involves the radii of subgraphs, as follows.

Lemma 1.17 (Brouwer et al. [8], Godsil and Royle [27]). Let $G$ be a connected graph. If $H$ is a subgraph of $G$, then $\rho(H) \leq \rho(G)$ and $q(H) \leq q(G)$, with strict inequalities in case $H$ is a proper subgraph of $G$.

We will also frequently use the following two known inequalities for $\rho(G)$ and $q(G)$, respectively.

Lemma 1.18 (Nikiforov [50]). Let $G$ be a graph on $n$ vertices and $m$ edges with minimum degree $\delta$. Then $\rho(G) \leq \frac{\delta-1}{2}+\sqrt{2 m-n \delta+\frac{(\delta+1)^{2}}{4}}$.

Lemma 1.19 (Feng et al. [23], Yu et al. [64]). Let $G$ be a connected graph on $n$ vertices and $m$ edges. Then $q(G) \leq \frac{2 m}{n-1}+n-2$.

In conjunction with Lemma 1.18, we also use the following property.

Lemma 1.20 (Hong et al. [31], Nikiforov [50]). For nonnegative integers $p$ and $q$ with $2 q \leq p(p-1)$ and $0 \leq x \leq p-1$, the function $f(x)=\frac{x-1}{2}+$ $\sqrt{2 q-p x+\frac{(1+x)^{2}}{4}}$ is decreasing with respect to $x$. 


\section{Chapter 2}

\section{Sufficient spectral radius conditions for hamiltonicity}

During the last decade several research groups have published results on sufficient conditions for the hamiltonicity of graphs in terms of their spectral radius and their signless Laplacian spectral radius. Here we extend some of these results. All of our results involve the characterization of the exceptional graphs, i.e., all the nonhamiltonian graphs that satisfy the condition. The proofs of our main results are based on the Bondy-Chvátal closure, a degree sequence condition due to Chvátal, and the Kelmans transformation that we explained in the introductory chapter.

\subsection{Introduction}

We start by listing a number of existing hamiltonicity results. To put our results in the right context, we divided the results into two subsections, in which we give a brief summary of a number of sufficient conditions for hamiltonicity in terms of the spectral radius, and signless Laplacian spectral radius, respectively, that were obtained in the last decade. Our aim is to extend these results. 


\subsubsection{Sufficient conditions in terms of the spectral radius}

As we mentioned in Chapter 1, Fiedler and Nikiforov [25] presented the sufficient condition for hamiltonicity that is stated in Theorem 1.3. This work spurred the interest of several research groups.

In 2015, Ning and Ge [53] obtained the following closely related sufficient condition for a graph $G$ with minimum degree $\delta(G) \geq 2$ to be hamiltonian.

Theorem 2.1 (Ning and Ge [53]). Let $G$ be a graph of order $n \geq 14$ with $\delta(G) \geq 2$. If $\rho(G) \geq \rho\left(K_{2} \vee\left(K_{n-4}+2 K_{1}\right)\right)$, then $G$ is hamiltonian unless $G=K_{2} \vee\left(K_{n-4}+2 K_{1}\right)$.

The graph $K_{2} \vee\left(K_{n-4}+2 K_{1}\right)$ is clearly not hamiltonian, since deleting the two vertices corresponding to $K_{2}$ results in three components in the remaining graph, so it is not 1-tough. Excluding a family of four classes of graphs that are not 1-tough, Benediktovich [2] obtained the following extension of the result of Ning and Ge.

Theorem 2.2 (Benediktovich [2]). Let $G$ be a graph of order $n \geq 9$ with $\delta(G) \geq 2$. If $\rho(G) \geq n-3$, then $G$ is hamiltonian unless $G \in\left\{K_{4} \vee 5 K_{1}, K_{3} \vee\right.$ $\left.\left(K_{1,4}+K_{1}\right), K_{1} \vee\left(K_{n-3}+K_{2}\right), K_{2} \vee\left(K_{n-4}+2 K_{1}\right)\right\}$.

By imposing the minimum degree condition $\delta(G) \geq k$, for general integers $k \geq 1$, Li and Ning [41] established the following result.

Theorem 2.3 (Li and Ning [41]). Let $k$ be an integer, and let $G$ be a graph of order $n$. If $\delta(G) \geq k \geq 1$ and $\rho(G) \geq \rho\left(K_{k} \vee\left(K_{n-2 k}+k K_{1}\right)\right)$, where $n \geq \max \left\{6 k+5,\left(k^{2}+6 k+4\right) / 2\right\}$, then $G$ is hamiltonian unless $G=K_{k} \vee$ $\left(K_{n-2 k}+k K_{1}\right)$.

Recently, Nikiforov [52] extended and strengthened Theorem 2.3 in the following sense.

Theorem 2.4 (Nikiforov [52]). Let $G$ be a graph of order $n$ with $\delta(G) \geq k$. If $k \geq 2, n \geq k^{3}+k+4$ and $\rho(G) \geq n-k-1$, then $G$ is hamiltonian unless $G=K_{1} \vee\left(K_{n-k-1}+K_{k}\right)$ or $G=K_{k} \vee\left(K_{n-2 k}+k K_{1}\right)$. 
More recently, Ge and Ning [26] showed that the statement in the above theorem due to Nikiforov also holds for $k \geq 1$ and $n \geq \max \left\{\frac{1}{2} k^{3}+k+\frac{5}{2}, 6 k+\right.$ $5\}$.

Motivated by the above results, in this chapter we continue the study of sufficient conditions for hamiltonicity in terms of the spectral radius. Our main result in this section involves a slightly different type of lower bound on the spectral radius, and it involves the well-known Bondy-Chvátal closure [5]. We postpone all the proofs of our contributions to Section 2.3.

Theorem 2.5. Let $G$ be a graph of order $n \geq 6 k^{2}+4 k+2$ with $\delta(G) \geq k \geq 1$. If $\rho(G)>\frac{k-1}{2}+\sqrt{n^{2}-(3 k+1) n+\frac{(k+1)^{2}-4}{4}}$, then $G$ is hamiltonian unless $c l_{n}(G)=K_{1} \vee\left(K_{n-k-1}+K_{k}\right)$ or $c l_{n}(G)=K_{k} \vee\left(K_{n-2 k}+k K_{1}\right)$.

In the above statement, $c l_{n}(G)$ denotes the Bondy-Chvátal closure of $G$, obtained from $G$ by recursively joining pairs of non-adjacent vertices by an edge whose degree sum is at least $n$ (in the current graph) until no such pair remains. The main results in [5] show that $c l_{n}(G)$ is well-defined and that $c l_{n}(G)$ is hamiltonian if and only if $G$ is hamiltonian.

It is easy to check that $n-k-1>\frac{k-1}{2}+\sqrt{n^{2}-(3 k+1) n+\frac{(k+1)^{2}-4}{2}}$. Hence Theorem 2.5 extends Theorem 2.4, in the sense that the lower bound on $\rho(G)$ is generally better.

We also obtained the following result for graphs on $n \geq 5$ vertices with $\delta(G) \geq 1$.

Theorem 2.6. Let $G$ be a graph with $n \geq 5$ and $\delta(G) \geq 1$. If $\rho(G)>$ $\sqrt{n^{2}-4 n}$, then $G$ is hamiltonian or $G \in\left\{G_{1}, G_{1}^{1}, G_{1}^{2}, G_{3}^{6}, G_{3}^{9}, G_{3}^{17}, G_{3}^{18}, G_{3}^{21}, G_{3}^{22}, G_{3}^{24}, G_{3}^{26}\right\}$.

Here, $G_{1}=K_{1} \vee\left(K_{n-2}+K_{1}\right)$, and $G_{1}^{1}$ and $G_{1}^{2}$ are obtained from $G_{1}$ by deleting one edge (as depicted in Figure 2.1 in Section 2.3). The other exceptional graphs in the above theorem belong to a larger set of graphs on 5-7 vertices that are defined in Section 2.3 and depicted in Figures 2.3-2.5. Note that our result is an improvement of Ge and Ning's result [26] in the case $k=1$.

We now turn to sufficient conditions in terms of the signless Laplacian spectral radius, and we start again with a brief summary of related results. 


\subsubsection{Sufficient conditions in terms of the signless Laplacian spec- tral radius}

For the signless Laplacian radius, several sufficient conditions for hamiltonian properties have appeared in [46], [64], and [66].

We start with the following results due to Yu and Fan [64], and Liu et al. [46], respectively.

Theorem 2.7 (Yu and Fan [64]). Let $G$ be a graph of order $n \geq 3$. If $q(G)>$ $2 n-4$, then $G$ is hamiltonian unless $G=K_{2} \vee 3 K_{1}$ or $G=K_{1} \vee\left(K_{n-2}+K_{1}\right)$.

Theorem 2.8 (Liu, Shiu and Xue [46]). Let $G$ be a graph of order $n \geq 4$ with $\delta(G) \geq 2$. If $q(G) \geq 2 n-5+\frac{3}{n-1}$, then $G$ is hamiltonian unless $G=K_{3} \vee 4 K_{1}$ or $G=K_{2} \vee 3 K_{1}$.

By imposing the minimum degree condition $\delta(G) \geq k$, for general integers $k \geq 1$, Li and Ning [41] established the following counterpart of Theorem 2.3.

Theorem 2.9 (Li and Ning [41]). Let $k$ be an integer, and let $G$ be a graph of order $n$. If $\delta(G) \geq k \geq 1$ and $q(G) \geq q\left(K_{k} \vee\left(K_{n-2 k}+k K_{1}\right)\right)$, where $n \geq$ $\max \left\{6 k+5,\left(3 k^{2}+5 k+4\right) / 2\right\}$, then $G$ is hamiltonian unless $G=K_{k} \vee\left(K_{n-2 k}+\right.$ $\left.k K_{1}\right)$.

The most recent result in this area that we are aware of is the following result due to Li et al. [40].

Theorem 2.10 (Li, Liu and Peng [40]). Let $k>1$ be an integer, let $n \geq$ $k^{4}+k^{3}+4 k^{2}+k+6$, and let $G$ be a connected graph of order $n$ with $\delta(G) \geq k$. If $q(G) \geq 2(n-k-1)$, then $G$ is hamiltonian unless $G \in \mathscr{M}_{1}(n, k)$ or $G \in$ $\mathscr{L}_{1}(n, k)$.

This result involves the two exceptional classes $\mathscr{M}_{1}(n, k)$ and $\mathscr{L}_{1}(n, k)$ that we will not define here. We refer the interested reader to [40] for the definitions.

Our main result in this section is the following counterpart of Theorem 2.5. 
Theorem 2.11. Let $G$ be a graph of order $n \geq 6 k^{2}+4 k+3$ with $\delta(G) \geq k \geq 1$. If $q(G)>2 n-2 k-2-\frac{2 k+1}{n-1}$, then $G$ is hamiltonian unless $c l_{n}(G)=K_{1} \vee$ $\left(K_{n-k-1}+K_{k}\right)$ or $c l_{n}(G)=K_{k} \vee\left(K_{n-2 k}+k K_{1}\right)$.

It is obvious that the lower bound condition on $q(G)$ of Theorem 2.11 is weaker than that of Theorem 2.10. Hence Theorem 2.11 strengthens Theorem 2.10 in that sense.

We also obtained the following result.

Theorem 2.12. Let $G$ be a graph of order $n \geq 6$ with $\delta(G) \geq 1$. If $q(G)>2 n-$ $4-\frac{3}{n-1}$, then $G$ is hamiltonian unless $G \in\left\{G_{1}, G_{1}^{1}, G_{1}^{2}, G_{3}^{6}, G_{3}^{8}, G_{3}^{9}, G_{3}^{17}, G_{3}^{21}\right.$, $\left.G_{3}^{23}, G_{3}^{26}\right\}$.

Here, as in Theorem 2.6, $G_{1}=K_{1} \vee\left(K_{n-2}+K_{1}\right)$, and $G_{1}^{1}$ and $G_{1}^{2}$ are obtained from $G_{1}$ by deleting one edge (as depicted in Figure 2.1). The other exceptional graphs in the above theorem belong to a larger set of graphs on 5-7 vertices that are defined in Section 2.3 and depicted in Figures 2.2-2.5. Noting that $K_{n-2} \varsubsetneqq G_{1}$, using Lemma 1.17 from Chapter 1 , we obtain that $q\left(G_{1}\right)>q\left(K_{n-2}\right)=2 n-4$. Therefore our result improves Theorem 2.9 in the case $k=1$.

The rest of the chapter is organized as follows. In Section 2.2, we introduce one known degree sequence result on hamiltonicity and the Bondy and Chvátal closure. In Section 2.3, we present the proofs of our results.

\subsection{Two useful lemmas}

In this section, we recall two known results on hamiltonicity that date back to the 1970s. The first one is a well-known degree sequence result due to Chvátal [12]. Here, the degrees of the $n$ vertices of a graph $G$ are ordered in a non-decreasing way as a degree sequence $\left(d_{1}, d_{2}, \ldots, d_{n}\right)$. We say that a sequence of non-decreasing integers is graphical if there exists a graph with that degree sequence.

Lemma 2.13 (Chvátal [12]). Let $G$ be a graph on $n \geq 3$ vertices with degree sequence $\left(d_{1}, d_{2}, \ldots, d_{n}\right)$. If there is no integer $k<n / 2$ such that $d_{k} \leq k$ and $d_{n-k} \leq n-k-1$, then $G$ is hamiltonian. 
The second result is a result due to Bondy and Chvátal [5] that we have mentioned earlier. It deals with the closure $c l_{n}(G)$ obtained from a graph $G$ by recursively adding edges between non-adjacent pairs of vertices with degree sum at least $n$, until no such pair remains in the resulting graph. It is easy to check and proved in [5] that $c l_{n}(G)$ is well-defined (unique). For our purposes the following statement is relevant.

Lemma 2.14 (Bondy and Chvátal [5]). A graph $G$ is hamiltonian if and only if $\mathrm{cl}_{n}(G)$ is hamiltonian.

\subsection{The proofs of our results}

The basis of the proofs of Theorem 2.5 and Theorem 2.11 is the following structural result.

Theorem 2.15. Let $G$ be a graph of order $n \geq 6 k^{2}+4 k+2$ with $\delta(G) \geq k \geq 1$. If

$$
e(G) \geq \frac{n^{2}-(2 k+1) n}{2},
$$

then $G$ is hamiltonian unless $c l_{n}(G)=K_{1} \vee\left(K_{k}+K_{n-k-1}\right)$ or $c l_{n}(G)=K_{k} \vee$ $\left(K_{n-2 k}+k K_{1}\right)$.

Proof. Let $H=c l_{n}(G)$, and let $H$ have degree sequence $\left(d_{1}, d_{2}, \ldots, d_{n}\right)$, where $d_{1} \leq d_{2} \leq \ldots \leq d_{n}$ and $d_{i}=d\left(v_{i}\right)$ for all $i \in\{1,2, \ldots, n\}$. The main consequence of this assumption is that $d_{i}+d_{j}<n$ for every two non-adjacent vertices $v_{i}$ and $v_{j}$. Suppose $G$ is not hamiltonian. By Lemma $2.14, H$ is not hamiltonian. Then, by Lemma 2.13, there is an integer $s<n / 2$ such that $d_{s} \leq s$ and $d_{n-s} \leq n-s-1$. Then

$$
\begin{aligned}
2 e(H) & =\sum_{i=1}^{n} d_{i} \\
& \leq s \cdot s+(n-2 s)(n-s-1)+s(n-1) \\
& =n^{2}-(2 s+1) n+3 s^{2}+s .
\end{aligned}
$$


Thus $n^{2}-(2 k+1) n \leq 2 e(H) \leq n^{2}-(2 s+1) n+3 s^{2}+s$, i.e., $(2 k-2 s) n+3 s^{2}+s \geq$ 0 . Let $f(x)=(2 k-2 x) n+3 x^{2}+x$. So $f(s) \geq 0$. The rest of the proof is based on three claims.

Claim 1. $s=k$ and $d_{1}=d_{2}=\ldots=d_{k}=k$.

Proof of Claim 1. Suppose that $s \geq k+1$. Then, since $f(x)$ is convex in $x$,

$$
\begin{aligned}
f(s) & \leq \max \left\{f(k+1), f\left(\frac{n-1}{2}\right)\right\} \\
& =\max \left\{3 k^{2}+7 k+4-2 n,-\frac{n^{2}}{4}+2 k n+\frac{1}{4}\right\} \\
& <0,
\end{aligned}
$$

a contradiction. Thus $s=k$ and $d_{1}=d_{2}=\ldots=d_{k}=k$.

Claim 2. $H\left[\left\{v_{k+1}, \ldots, v_{n}\right\}\right]=K_{n-k}$.

Proof of Claim 2. Firstly, we show $d_{k+1} \geq n-3 k^{2}-2 k-1$. In fact, if $d_{k+1}<$ $n-3 k^{2}-2 k-1$, then

$$
\begin{aligned}
2 e(H) & =\sum_{i=1}^{k} d_{i}+d_{k+1}+\sum_{i=k+2}^{n-k} d_{i}+\sum_{i=n-k+1}^{n} d_{i} \\
& <k \cdot k+n-3 k^{2}-2 k-1+(n-2 k-1)(n-k-1)+k(n-1) \\
& =n^{2}-(2 k+1) n,
\end{aligned}
$$

a contradiction. Thus $d_{i} \geq n-3 k^{2}-2 k-1$ for $k+1 \leq i \leq n$.

Suppose that $H\left[\left\{v_{k+1}, \ldots, v_{n}\right\}\right] \neq K_{n-k}$. Then there exist two non-adjacent distinct vertices $v_{i}, v_{j}$, where $k+1 \leq i, j \leq n$. Then we have

$$
d_{i}+d_{j} \geq 2\left(n-3 k^{2}-2 k-1\right) \geq n,
$$

a contradiction.

Let $X_{1}=\left\{v_{1}, \ldots, v_{k}\right\}$. Since $d_{1}=\ldots=d_{k}=k$ and $\left|X_{1}\right|=k$, every vertex in $X_{1}$ must have a neighbor and has at most $k$ neighbors in $\left\{v_{k+1}, \ldots, v_{n}\right\}$. 
Let $X_{2} \subseteq\left\{v_{k+1}, \ldots, v_{n}\right\}$ be the neighbors of vertices in $X_{1}$. Set $\left|X_{2}\right|=\ell$. So we have $1 \leq \ell \leq k$.

Claim 3. $H\left[X_{1}, X_{2}\right]=K_{k, \ell}$.

Proof of Claim 3. Suppose that $H\left[X_{1}, X_{2}\right] \neq K_{k, \ell}$. Then there exist two distinct vertices $u, v \in X_{1}$, and a vertex $w \in X_{2}$ such that $w \in N_{X_{2}}(u) \backslash N_{X_{1}}(v)$. Since $d_{H}(w) \geq n-k$, we have

$$
d_{H}(v)+d_{H}(w) \geq k+n-k=n,
$$

a contradiction. Hence $H\left[X_{1}, X_{2}\right]=K_{k, \ell}$.

Combining the above three claims, if $\ell=1$, then one easily concludes that $H=K_{1} \vee\left(K_{k}+K_{n-k-1}\right)$, and if $\ell=k$, that $H=K_{k} \vee\left(K_{n-2 k}+k K_{1}\right)$. For the case that $2 \leq \ell \leq k-1$, it is implicitly shown in the proof of Lemma 2 in [41], that $H$ is hamiltonian, a contradiction. We refrain from repeating these arguments here.

This completes the proof of Theorem 2.15.

Now it is a relatively easy exercise to deliver the proofs of Theorem 2.5 and Theorem 2.11.

Proof of Theorem 2.5. By Lemmas 1.18 and 1.20, and the fact that $\delta(G) \geq k$, we have $\rho(G) \leq \frac{k-1}{2}+\sqrt{2 e(G)-k n+\frac{(k+1)^{2}}{4}}$. Since

$$
\rho(G)>\frac{k-1}{2}+\sqrt{n^{2}-(3 k+1) n+\frac{(k+1)^{2}-4}{4}},
$$

we have $e(G) \geq \frac{n^{2}-(2 k+1) n}{2}$. Supposing that $G$ is not hamiltonian, using Theorem 2.15, we obtain that $c l_{n}(G)=K_{1} \vee\left(K_{k}+K_{n-k-1}\right)$ or $c l_{n}(G)=$ $K_{k} \vee\left(K_{n-2 k}+k K_{1}\right)$.

Proof of Theorem 2.11. Suppose $G$ is not hamiltonian. Using Lemma 1.19, we have

$$
2 n-2 k-2-\frac{2 k+1}{n-1}<q(G) \leq \frac{2 e(G)}{n-1}+n-2,
$$


thus $e(G) \geq \frac{n^{2}-(2 k+1) n}{2}$. Using Theorem 2.15, we conclude that $c l_{n}(G)=$ $K_{1} \vee\left(K_{k}+K_{n-k-1}\right)$ or $c l_{n}(G)=K_{k} \vee\left(K_{n-2 k}+k K_{1}\right)$.

Before we continue with the proofs of our other results, we first introduce a number of graphs that appear in the formulation of these results and in our next auxiliary result. We will mainly refer to the figures that follow, and not give rigorous definitions of these graphs. As we mentioned before $G_{1}=K_{1} \vee\left(K_{n-2}+K_{1}\right)$, and $G_{1}^{1}$ and $G_{1}^{2}$ are obtained from $G_{1}$ by deleting one edge, as depicted in Figure 2.1, in which the ellipses indicate a $K_{n-1}$ of sufficiently large order. The labels of the vertices will be used to indicate an equitable partition in the later proofs.
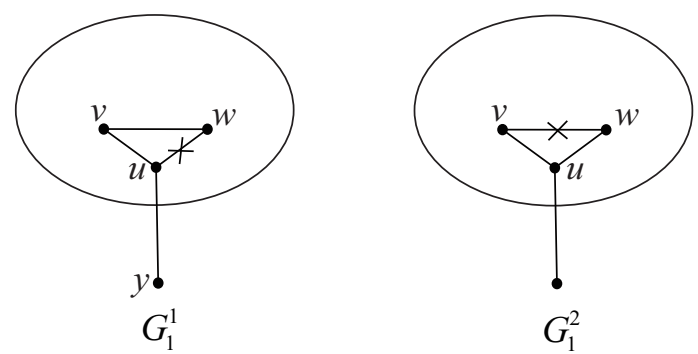

Figure 2.1: The graphs $G_{1}^{1}$ and $G_{1}^{2}$.

The graphs $G_{2}^{1}-G_{2}^{5}$ are obtained in a similar way from $G_{1}=K_{1} \vee\left(K_{n-2}+\right.$ $K_{1}$ ) by deleting two edges, as indicated in Figure 2.2, in which the ellipses again indicate a $K_{n-1}$ of sufficiently large order, and the labels will be used later.

Next to the infinite classes of graphs that have been sketched in Figures 2.1 and 2.2, we need to define a lot of small graphs on 5-7 vertices. The graphs $G_{3}^{1}-G_{3}^{6}$ on 7 vertices have been drawn in Figure 2.3, in which the numerical labels indicate the degrees of the vertices. Similarly, the graphs $G_{3}^{7}-G_{3}^{16}$ on 6 vertices have been drawn in Figure 2.4, in which the numerical labels again indicate the degrees of the vertices. Finally, the graphs $G_{3}^{17}-G_{3}^{26}$ on 5 vertices have been drawn in Figure 2.5, in which the numerical labels again indicate the degrees of the vertices. 


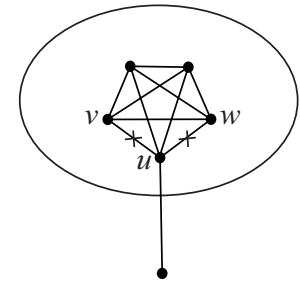

$G_{2}^{1}$

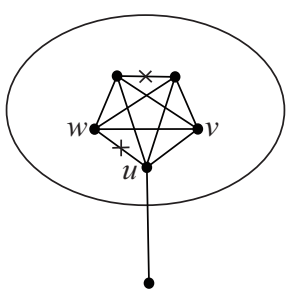

$G_{2}^{2}$

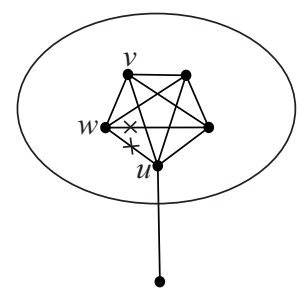

$G_{2}^{3}$

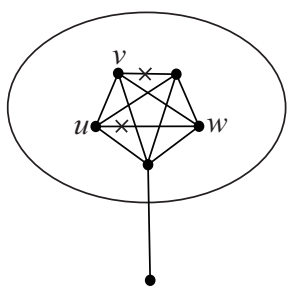

$G_{2}^{4}$

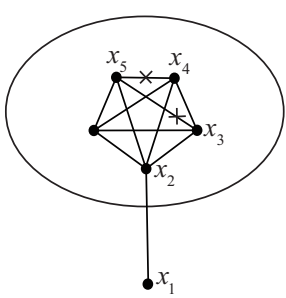

$G_{2}^{5}$

FIGURE 2.2: The graphs $G_{2}^{1}-G_{2}^{5}$.
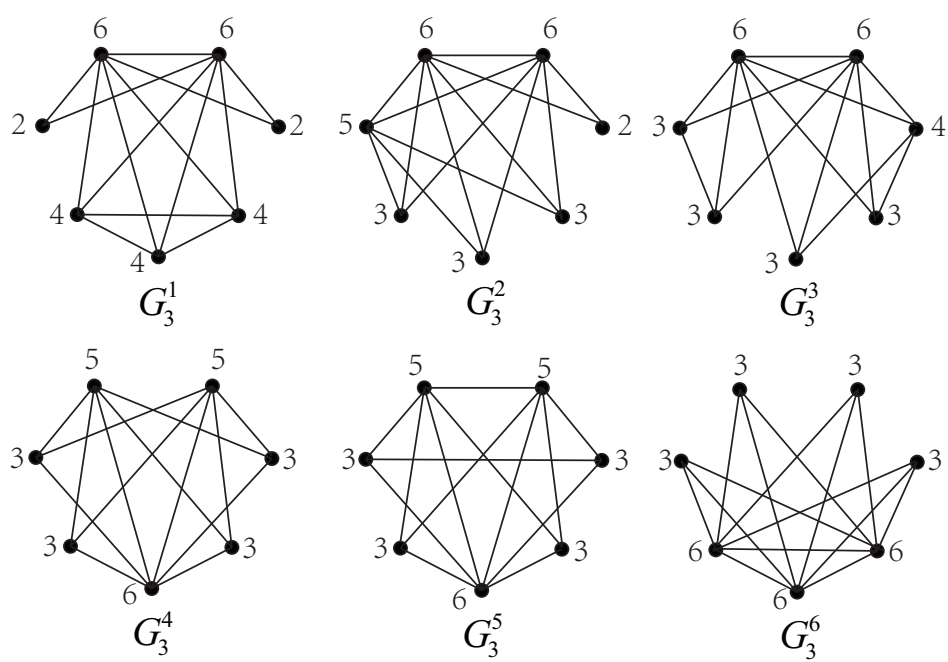

Figure 2.3: The graphs $G_{3}^{1}-G_{3}^{6}$ together with their vertex degrees. 

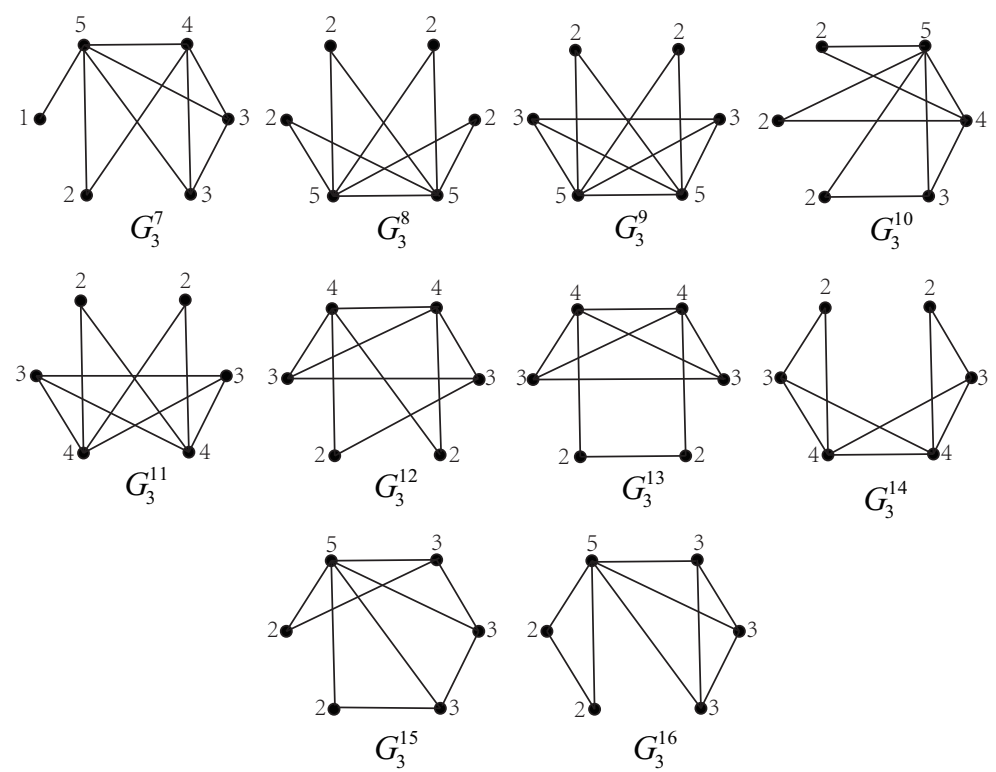

FIgURE 2.4: The graphs $G_{3}^{7}-G_{3}^{16}$ together with their vertex degrees.
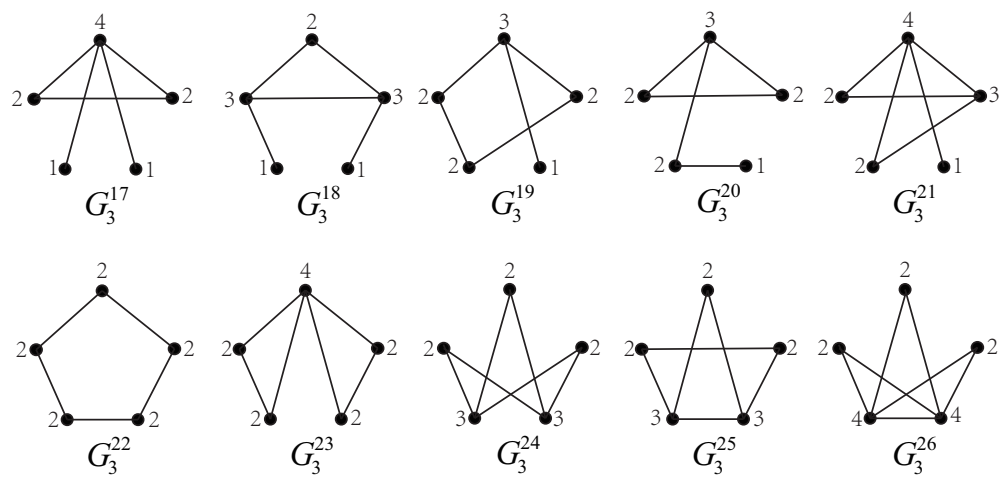

Figure 2.5: The graphs $G_{3}^{17}-G_{3}^{26}$ together with their vertex degrees. 
We note here that some of the depicted graphs in Figures 2.3-2.5 are hamiltonian, while others are not, and that some have a complete closure, while others have not. These properties are relatively easy to check because the graphs have a small number of vertices.

One of the key ingredients in the proofs of Theorems 2.6 and 2.12 is the following structural result.

Theorem 2.16. Let $G$ be a graph of order $n \geq 5$ with $\delta(G) \geq 1$. If

$$
e(G) \geq\left(\begin{array}{c}
n-1 \\
2
\end{array}\right)-1,
$$

then $G$ is hamiltonian unless $G \in\left\{G_{1}, G_{1}^{1}, G_{1}^{2}, G_{2}^{1}, G_{2}^{2}, G_{2}^{3}, G_{2}^{4}, G_{2}^{5}, G_{3}^{1}, G_{3}^{2}, G_{3}^{4}\right.$, $\left.G_{3}^{6}, G_{3}^{7}, G_{3}^{8}, G_{3}^{9}, G_{3}^{10}, G_{3}^{11}, G_{3}^{16}, G_{3}^{17}, G_{3}^{18}, G_{3}^{19}, G_{3}^{20}, G_{3}^{21}, G_{3}^{23}, G_{3}^{24}, G_{3}^{26}\right\}$.

Proof. Suppose that $G$ is not hamiltonian and that $G$ has degree sequence $\left(d_{1}, d_{2}, \ldots, d_{n}\right)$, where $d_{1} \leq d_{2} \leq \cdots \leq d_{n}$ and $d_{i}=d\left(v_{i}\right)$ for $i \in\{1,2, \ldots, n\}$. By Lemma 2.13, there exists an integer $k<n / 2$ such that $d_{k} \leq k$ and $d_{n-k} \leq$ $n-k-1$. Thus

$$
\begin{aligned}
n^{2}-3 n \leq 2 e(G)=\sum_{i=1}^{n} d_{i} & \leq k \cdot k+(n-2 k)(n-k-1)+k(n-1) \\
& =n^{2}-(2 k+1) n+3 k^{2}+k .
\end{aligned}
$$

Since $n \geq 2 k+1$, we have $k^{2}-3 k-2 \leq 0$, i.e., $1 \leq k \leq 3$. We distinguish three main cases according to the value of $k$.

Case 1. $k=1$. Then $d_{1}=1, d_{n-1} \leq n-2$, so $\left(\begin{array}{c}n-1 \\ 2\end{array}\right)-1 \leq e(G) \leq$ $\left(\begin{array}{c}n-1 \\ 2\end{array}\right)+1$. If $e(G)=\left(\begin{array}{c}n-1 \\ 2\end{array}\right)+1$, then $d_{1}=1, d_{2}=\ldots=d_{n-1}=n-2$, $d_{n}=n-1$, which implies that $G=K_{1} \vee\left(K_{n-2}+K_{1}\right)=G_{1}$. If $e(G)=$ $\left(\begin{array}{c}n-1 \\ 2\end{array}\right)$, then $G$ is a graph obtained from $G_{1}$ by deleting one edge. Since $\delta(G) \geq 1, G \in\left\{G_{1}^{1}, G_{1}^{2}\right\}$ (See Figure 2.1). If $e(G)=\left(\begin{array}{c}n-1 \\ 2\end{array}\right)-1$, then $G$ is a graph obtained from $G_{1}$ by deleting two edges. Since $\delta(G) \geq 1$, $G \in\left\{G_{2}^{1}, G_{2}^{2}, G_{2}^{3}, G_{2}^{4}, G_{2}^{5}\right\}$ (See Figure 2.2). 
Case 2. $k=2$. Then $d_{2} \leq 2, d_{n-2} \leq n-3$, and so $n^{2}-3 n \leq 2 e(G) \leq$ $n^{2}-5 n+14$. Since $n \geq 2 k+1=5$, we have $5 \leq n \leq 7$. We distinguish three subcases.

Subcase 2.1. $n=7$. Then $d_{5} \leq 4$ and $e(G)=14$. If $d_{1}=1$, then $d_{6} \leq$ 5 , and there is no graphical sequence satisfying these conditions. Hence $d_{1}=d_{2}=2$. Then the degree sequence is $(2,2,4,4,4,6,6)$, which implies $G=K_{2} \vee\left(2 K_{1}+K_{3}\right)=G_{3}^{1}$ (See Figure 2.3).

Subcase 2.2. $n=6$. Then $d_{4} \leq 3$ and $9 \leq e(G) \leq 10$. If $d_{1}=1$, then $d_{5} \leq 4$. In this case, we can get only one graphical sequence $(1,2,3,3,4,5)$, which implies $G=G_{3}^{7}$ (See Figure 2.4 for this graph and the other graphs in this subcase). If $d_{1}=d_{2}=2$, then we have $d_{5}+d_{6}=2 m-\sum_{i=1}^{4} d_{i} \geq 18-10=8$. Also note that $\sum d_{i}$ is even and $18 \leq \sum d_{i} \leq 20$. If $d_{5}=d_{6}=5$, then the only graphical sequences are $(2,2,2,2,5,5)$ and $(2,2,3,3,5,5)$. The first sequence implies that $G=K_{2} \vee 4 K_{1}=G_{3}^{8}$ and the second sequence implies that $G=K_{2} \vee\left(K_{2}+2 K_{1}\right)=G_{3}^{9}$. If $d_{5}=4$ and $d_{6}=5$, then the only graphical sequence is $(2,2,2,3,4,5)$, which implies that $G=G_{3}^{10}$. If $d_{5}=d_{6}=4$, then the only graphical sequence is $(2,2,3,3,4,4)$, which implies that $G \in\left\{G_{3}^{11}, G_{3}^{12}, G_{3}^{13}, G_{3}^{14}\right\}$. Note that each of $\left\{G_{3}^{12}, G_{3}^{13}, G_{3}^{14}\right\}$ is hamiltonian, hence $G=G_{3}^{11}$. If $d_{5}=3$ and $d_{6}=5$, then the only graphical sequence is $(2,2,3,3,3,5)$, which implies that $G \in\left\{G_{3}^{15}, G_{3}^{16}\right\}$. Note that $G_{3}^{15}$ is hamiltonian. Hence $G=G_{3}^{16}$.

Subcase 2.3. $n=5$. Then $d_{3} \leq 2$ and $5 \leq e(G) \leq 7$. If $d_{1}=d_{2}=d_{3}=1$, then $d_{4} \leq 3$, and there is no graphical sequence. If $d_{1}=d_{2}=1$ and $d_{3}=2$, then $d_{4} \leq 3$. In this case there are two graphical sequences $(1,1,2,2,4)$ and $(1,1,2,3,3)$, which implies $G=G_{3}^{17}$ and $G=G_{3}^{18}$, respectively (See Figure 2.5 for these two graphs and the other graphs in this subcase). If $d_{1}=1$ and $d_{2}=d_{3}=2$, then there are two graphical sequences $(1,2,2,2,3)$ and $(1,2,2,3,4)$. The first sequence implies that $G \in\left\{G_{3}^{19}, G_{3}^{20}\right\}$. The second sequence implies that $G=G_{3}^{21}$. If $d_{1}=d_{2}=d_{3}=2$, then $4 \leq d_{4}+d_{5} \leq 8$. If $d_{4}=d_{5}=2$, then the graphical sequence is $(2,2,2,2,2)$, which implies that $G=C_{5}=G_{3}^{22}$, a contradiction. If $d_{4}=2$ and $d_{5}=4$, then the graphical sequence is $(2,2,2,2,4)$, which implies that $G=K_{1} \vee 2 K_{2}=G_{3}^{23}$. If $d_{4}=$ $d_{5}=3$, then the graphical sequence is $(2,2,2,3,3)$, which implies that $G \in$ 
$\left\{G_{3}^{24}, G_{3}^{25}\right\}$. Note that $G_{3}^{25}$ is hamiltonian. Hence $G=G_{3}^{24}$. If $d_{4}=d_{5}=4$, then the graphical sequence is $(2,2,2,4,4)$, which implies that $G=K_{2} \vee$ $3 K_{1}=G_{3}^{26}$. This completes Case 2 .

Case 3. $k=3$. Then $d_{3} \leq 3, d_{n-3} \leq n-4$ and $n^{2}-3 n \leq 2 e(G) \leq n^{2}-7 n+30$. Since $n \geq 2 k+1=7$, we obtain that $n=7$. Hence $d_{4} \leq 3$ and $14 \leq$ $e(G) \leq 15$. If $d_{1}=1$, then $d_{6} \leq 5$ and there is no graphical sequence. If $d_{1}=d_{2}=d_{3}=d_{4}=2$, then $d_{5} \leq 5$, and there is also no graphical sequence. If $d_{1}=d_{2}=d_{3}=2$ and $d_{4}=3$, then $d_{5} \leq 5$ and there is again no graphical sequence. If $d_{1}=d_{2}=2$ and $d_{3}=d_{4}=3$, then $d_{5} \leq 5$ and there is no graphical sequence either. If $d_{1}=2$ and $d_{2}=d_{3}=d_{4}=3$, then $d_{5} \leq 5$ and the only graphical sequence is $(2,3,3,3,5,6,6)$, which implies $G=K_{2} \vee\left(K_{1,3}+K_{1}\right)=G_{3}^{2}$. If $d_{1}=d_{2}=d_{3}=d_{4}=3$, then $d_{5} \leq 6$. Note that $28 \leq \sum_{i=1}^{7} d_{i} \leq 30$ and $\sum_{i=1}^{7} d_{i}$ is an even number. Hence the only graphical sequences are $(3,3,3,3,4,6,6),(3,3,3,3,5,5,6)$ and $(3,3,3,3,6,6,6)$. From the first sequence, we obtain that $G=G_{3}^{3}$, which is hamiltonian, a contradiction. For the second sequence, there are two possibilities: if $v_{5}$ is not adjacent to $v_{6}$, then $G=K_{1} \vee K_{2,4}=G_{3}^{4}$. If $v_{5}$ is adjacent to $v_{6}$, then $G=G_{3}^{5}$. Noting that $G_{3}^{5}$ is hamiltonian, the second possibility yields a contradiction. From the third sequence, we obtain that $G=K_{3} \vee 4 K_{1}=G_{3}^{6}$.

This completes the proof of Theorem 2.16.

Next, we prove two lemmas in order to deduce lower bounds on the radii of the graphs of Figure 2.1 and upper bounds on the radii of the graphs of Figure 2.2, respectively.

Lemma 2.17. For $n \geq 3$ and $1 \leq i \leq 2$,

(i) $\rho\left(G_{1}^{i}\right)>\sqrt{n^{2}-4 n}$ and

(ii) $q\left(G_{1}^{i}\right)>2 n-4-\frac{3}{n-1}$.

Proof. Firstly, we claim that $\rho\left(G_{1}^{2}\right) \geq \rho\left(G_{1}^{1}\right)$ and $q\left(G_{1}^{2}\right) \geq q\left(G_{1}^{1}\right)$. Indeed, let $u, v, w$ be the three vertices corresponding to the labels in Figure 2.1. It is clear that $G_{1}^{2}=G_{1}^{1}-v w+u w$ (with the obvious meaning that we do not define formally). Using Lemmas 1.14 and 1.15 , it is clear that the claim holds. 
Hence it is sufficient to show only that $\rho\left(G_{1}^{1}\right)>\sqrt{n^{2}-4 n}$ and $q\left(G_{1}^{1}\right)>$ $2 n-4-\frac{3}{n-1}$.

Let us consider the following partition $\pi$ of $V\left(G_{1}^{1}\right)$ in $X_{1}=\{y\}, X_{2}=\{u\}$, $X_{3}=\{w\}$, and $X_{4}=V\left(G_{1}^{1}\right) \backslash\{y, u, w\}$. It is easy to check that this partition is equitable, and that the corresponding adjacency matrix and signless Laplacian matrix of its quotient $G_{1}^{1} / \pi$ are as follows:

$$
\begin{gathered}
A\left(G_{1}^{1} / \pi\right)=\left(\begin{array}{cccc}
0 & 1 & 0 & 0 \\
1 & 0 & 0 & n-3 \\
0 & 0 & 0 & n-3 \\
0 & 1 & 1 & n-4
\end{array}\right) . \\
Q\left(G_{1}^{1} / \pi\right)=\left(\begin{array}{cccc}
1 & 1 & 0 & 0 \\
1 & n-2 & 0 & n-3 \\
0 & 0 & n-3 & n-3 \\
0 & 1 & 1 & 2 n-6
\end{array}\right) .
\end{gathered}
$$

Let $f_{1}(x)=\operatorname{det}\left(x I_{4}-A\left(G_{1}^{1} / \pi\right)\right)$ and $g_{1}(x)=\operatorname{det}\left(x I_{4}-Q\left(G_{1}^{1} / \pi\right)\right)$ be the characteristic polynomials of $A\left(G_{1}^{1} / \pi\right)$ and $Q\left(G_{1}^{1} / \pi\right)$, respectively. Then, using the Laplace expansion for the calculation of the determinants, after some standard arithmetic we obtain:

$$
\begin{gathered}
f_{1}(x)=x^{4}-(n-4) x^{3}-(2 n-5) x^{2}+(n-4) x+n-3 ; \\
g_{1}(x)=x^{4}-(4 n-10) x^{3}+\left(5 n^{2}-25 n+30\right) x^{2}-\left(2 n^{3}-13 n^{2}+21 n\right) x \\
+2 n^{3}-20 n^{2}+66 n-72 .
\end{gathered}
$$

This implies $f_{1}\left(n-2-\frac{2}{n}\right)=-n-\frac{16}{n}-\frac{4}{n^{2}}+\frac{32}{n^{3}}+\frac{16}{n^{4}}+7$, and that $f_{1}\left(n-2-\frac{2}{n}\right)<0$ for $n \geq 3$. We also get that $g_{1}\left(2 n-4-\frac{3}{n-1}\right)=\frac{-11 n^{5}+95 n^{4}-267 n^{3}+215 n^{2}+100 n-51}{(n-1)^{4}}$, so that $g_{1}\left(2 n-4-\frac{3}{n-1}\right)<0$ for $n \geq 3$. Using Lemma 1.16, we conclude that $\rho\left(G_{1}^{1}\right)>n-2-\frac{2}{n}>\sqrt{n^{2}-4 n}$ and $q\left(G_{1}^{1}\right)>2 n-4-\frac{3}{n-1}$, confirming (i) and (ii), respectively. This completes the proof.

Lemma 2.18. For $n \geq 6$ and $1 \leq i \leq 5$, 
(i) $\rho\left(G_{2}^{i}\right)<\sqrt{n^{2}-4 n}$ and

(ii) $q\left(G_{2}^{i}\right)<2 n-4-\frac{3}{n-1}$.

Proof. Firstly, we claim that $\rho\left(G_{2}^{i}\right) \leq \rho\left(G_{2}^{5}\right)$ and $q\left(G_{2}^{i}\right) \leq q\left(G_{2}^{5}\right)$ for $1 \leq i \leq 4$. Indeed, let $u, v, w$ be the three vertices of $G_{2}^{i}$ corresponding to the labels in Figure 2.2 for $1 \leq i \leq 4$. For $1 \leq i \leq 4$, we have $N_{G_{2}^{i}}(v) \backslash N_{G_{2}^{i}}[u]=\{w\}$ and $N_{G_{2}^{i}}(u) \backslash N_{G_{2}^{i}}[v] \neq \emptyset$. It is easy to check that

$$
\begin{aligned}
& G_{2}^{3}=G_{2}^{1}-v w+u w, \\
& G_{2}^{4}=G_{2}^{2}-v w+u w, \\
& G_{2}^{5}=G_{2}^{4}-v w+u w, \\
& G_{2}^{5}=G_{2}^{3}-v w+u w .
\end{aligned}
$$

Using Lemmas 1.14 and 1.15, it is clear that the claim holds. Hence it is sufficient to show only that $\rho\left(G_{2}^{5}\right)<\sqrt{n^{2}-4 n}$ and $q\left(G_{2}^{5}\right)<2 n-4-\frac{3}{n-1}$.

Let us consider the following partition $\pi$ of $V\left(G_{2}^{5}\right)$ in $X_{1}=\left\{x_{1}\right\}, X_{2}=$ $\left\{x_{2}\right\}, X_{3}=\left\{x_{3}, x_{4}\right\}, X_{4}=\left\{x_{5}\right\}$, and $X_{5}=V\left(G_{2}^{5}\right) \backslash\left\{x_{1}, x_{2}, x_{3}, x_{4}, x_{5}\right\}$. It is easy to check that this partition is equitable, and that the corresponding adjacency matrix and signless Laplacian matrix of its quotient $G_{2}^{5} / \pi$ are as follows:

$$
\begin{gathered}
A\left(G_{2}^{5} / \pi\right)=\left(\begin{array}{ccccc}
0 & 1 & 0 & 0 & 0 \\
1 & 0 & 2 & 1 & n-5 \\
0 & 1 & 1 & 0 & n-5 \\
0 & 1 & 0 & 0 & n-5 \\
0 & 1 & 2 & 1 & n-6
\end{array}\right) . \\
Q\left(G_{2}^{5} / \pi\right)=\left(\begin{array}{ccccc}
1 & 1 & 0 & 0 & 0 \\
1 & n-1 & 2 & 1 & n-5 \\
0 & 1 & n-2 & 0 & n-5 \\
0 & 1 & 0 & n-4 & n-5 \\
0 & 1 & 2 & 1 & 2 n-8
\end{array}\right) .
\end{gathered}
$$


Let $f_{2}(x)=\operatorname{det}\left(x I_{5}-A\left(G_{2}^{5} / \pi\right)\right)$ and $g_{2}(x)=\operatorname{det}\left(x I_{5}-Q\left(G_{2}^{5} / \pi\right)\right)$ be the characteristic polynomials of $A\left(G_{2}^{5} / \pi\right)$ and $Q\left(G_{2}^{5} / \pi\right)$, respectively. Then

$$
f_{2}(x)=x^{5}-(n-5) x^{4}-(3 n-10) x^{3}-2 x^{2}+(3 n-13) x-n+5
$$

and

$$
\begin{aligned}
g_{2}(x) & =x^{5}-(5 n-14) x^{4}+\left(9 n^{2}-51 n+71\right) x^{3}-\left(7 n^{3}-58 n^{2}+150 n\right. \\
& -110) x^{2}+\left(2 n^{4}-19 n^{3}+51 n^{2}-108\right) x-2 n^{4}+28 n^{3} \\
& -146 n^{2}+336 n-288
\end{aligned}
$$

To complete the proof of (i), first of all we obtain the following derivatives of $f_{2}(x)$ with respect to $x$, by standard analysis:

$$
\begin{aligned}
f_{2}^{\prime}(x) & =5 x^{4}-4(n-5) x^{3}-3(3 n-10) x^{2}-4 x+3 n-13, \\
f_{2}^{(2)}(x) & =20 x^{3}-12(n-5) x^{2}-6(3 n-10) x-4, \\
f_{2}^{(3)}(x) & =60 x^{2}-24(n-5) x-6(3 n-10), \\
f_{2}^{(4)}(x) & =120 x-24(n-5), \\
f_{2}^{(5)}(x) & =120 .
\end{aligned}
$$

By substitution and simple but tedious calculations, for $n \geq 6$, we obtain

$$
\begin{aligned}
f_{2}\left(n-2-\frac{7}{2 n}\right)= & \frac{1}{2} n^{3}-\frac{11}{2} n^{2}+22 n-\frac{141}{4}-\frac{343}{4 n}+\frac{2989}{8 n^{2}}+\frac{343}{2 n^{3}} \\
& -\frac{12005}{16 n^{4}}-\frac{16807}{32 n^{5}} \\
> & \frac{1}{2} n^{3}-\frac{11}{2} n^{2}+22 n-41 \\
= & \frac{n}{2}(n-5)(n-6)+7 n-41>0, \\
f_{2}^{\prime}\left(n-2-\frac{7}{2 n}\right)= & n^{4}-5 n^{3}-10 n^{2}+60 n-\frac{1589}{4 n}-\frac{637}{2 n^{2}}+\frac{1715}{2 n^{3}} \\
& +\frac{12005}{16 n^{4}}+\frac{175}{2}
\end{aligned}
$$




$$
\begin{aligned}
& >n^{4}-5 n^{3}-10 n^{2}+60 n+16 \\
& =\left(n^{2}-4\right)(n-6)(n+1)+40 n-8>0, \\
f_{2}^{(2)}\left(n-2-\frac{7}{2 n}\right) & =8 n^{3}-30 n^{2}-78 n+\frac{378}{n}-\frac{735}{n^{2}}-\frac{1715}{2 n^{3}}+271 \\
> & 8 n^{3}-30 n^{2}-78 n+169 \\
& =2 n(2 n-12)(2 n+4)+2 n^{2}+18 n+169>0, \\
f_{2}^{(3)}\left(n-2-\frac{7}{2 n}\right) & =36 n^{2}-90 n+\frac{420}{n}+\frac{735}{n^{2}}-276 \\
& >36 n^{2}-90 n-276 \\
& =6(2 n+2)(3 n-18)+6(15 n-10)>0, \\
f_{2}^{(4)}\left(n-2-\frac{7}{2 n}\right) & =96 n-\frac{420}{n}-120>0, \\
f_{2}^{(5)}\left(n-2-\frac{7}{2 n}\right) & =120 .
\end{aligned}
$$

Hence, by the Fourier-Budan Theorem (See, e.g., [57]), there is no root of $f_{2}(x)$ in the interval $\left[n-2-\frac{7}{2 n},+\infty\right)$. Thus, using Lemma 1.16, we obtain that $\rho\left(G_{2}^{5}\right)<n-2-\frac{7}{2 n}<\sqrt{n^{2}-4 n}$.

To prove (ii), for the derivatives of $g_{2}(x)$ with respect to $x$, we obtain:

$$
\begin{aligned}
g_{2}^{\prime}(x)= & 5 x^{4}-4(5 n-14) x^{3}+3\left(9 n^{2}-51 n+71\right) x^{2}-2\left(7 n^{3}-58 n^{2}\right. \\
& +150 n-110)+\left(2 n^{4}-19 n^{3}+51 n^{2}-108\right), \\
g_{2}^{(2)}(x)= & 20 x^{3}-12(5 n-14) x^{2}+6\left(9 n^{2}-51 n+71\right) x-2\left(7 n^{3}-58 n^{2}\right. \\
& +150 n-110), \\
g_{2}^{(3)}(x)= & 60 x^{2}-24(5 n-14) x+6\left(9 n^{2}-51 n+71\right), \\
g_{2}^{(4)}(x)= & 120 x-24(5 n-14), \\
g_{2}^{(5)}(x)= & 120 .
\end{aligned}
$$

By substitution, for $n \geq 6$, we obtain

$$
g_{2}\left(2 n-4-\frac{3}{n-1}\right)=\frac{1}{(n-1)^{5}}\left(8 n^{8}-111 n^{7}+572 n^{6}-1327 n^{5}+1116 n^{4}\right.
$$




$$
\begin{aligned}
& \left.+587 n^{3}-1174 n^{2}-42 n+128\right) \\
& >\frac{1}{n^{5}}\left(8 n^{8}-111 n^{7}+572 n^{6}-1327 n^{5}+1116 n^{4}\right. \\
& \left.+587 n^{3}-1174 n^{2}-42 n+128\right) \\
& =8 n^{3}-111 n^{2}+572 n-1327+\frac{1116}{n} \\
& +\frac{587}{n^{2}}-\frac{1174}{n^{3}}-\frac{42}{n^{4}}+\frac{128}{n^{5}} \\
& >0 \\
& g_{2}^{\prime}\left(2 n-4-\frac{3}{n-1}\right)=\frac{1}{(n-1)^{4}}\left(2 n^{8}-15 n^{7}+29 n^{6}-48 n^{5}+425 n^{4}\right. \\
& \left.-1252 n^{3}+889 n^{2}+541 n-166\right) \\
& >\frac{1}{n^{4}}\left(2 n^{8}-15 n^{7}+29 n^{6}-48 n^{5}+425 n^{4}-1252 n^{3}\right. \\
& \left.+889 n^{2}+541 n-166\right) \\
& =2 n^{4}-15 n^{3}+29 n^{2}-48 n+425-\frac{1252}{n}+\frac{889}{n^{2}} \\
& +\frac{541}{n^{3}}-\frac{166}{n^{4}} \\
& >2 n^{4}-15 n^{3}+29 n^{2}-48 n+243 \\
& =n^{2}(n-2)(2 n-11)+(7 n-6)(n-6)+207>0 \text {, } \\
& g_{2}^{(2)}\left(2 n-4-\frac{3}{n-1}\right)=\frac{2\left(7 n^{6}-41 n^{5}+24 n^{4}+156 n^{3}-10 n^{2}-435 n+29\right)}{(n-1)^{3}} \\
& >\frac{2\left(7 n^{6}-41 n^{5}+24 n^{4}+156 n^{3}-10 n^{2}-435 n+29\right)}{n^{3}} \\
& =2\left[7 n^{3}-41 n^{2}+24 n+156-\frac{10}{n}-\frac{435}{n^{2}}+\frac{29}{n^{3}}\right] \\
& >2\left[7 n^{3}-41 n^{2}+24 n+142\right] \\
& =2\left[n(7 n-4)(n-6)+5 n^{2}+142\right]>0 \text {, } \\
& g_{2}^{(3)}\left(2 n-4-\frac{3}{n-1}\right)=\frac{6\left(9 n^{4}-37 n^{3}-6 n^{2}+99 n+25\right)}{(n-1)^{2}} \\
& >\frac{6\left(9 n^{4}-37 n^{3}-6 n^{2}+99 n+25\right)}{n^{2}}
\end{aligned}
$$




$$
\begin{aligned}
& =6\left(9 n^{2}-37 n-6+\frac{99}{n}+\frac{25}{n^{2}}\right) \\
& >6\left(9 n^{2}-37 n-6\right) \\
& =6[(9 n+1)(n-6)+16 n]>0 \\
g_{2}^{(4)}\left(2 n-4-\frac{3}{n-1}\right) & =24\left(5 n-\frac{15}{n-1}-6\right)>0 \\
g_{2}^{(5)}\left(2 n-4-\frac{3}{n-1}\right) & =120>0 .
\end{aligned}
$$

Hence, by the Fourier-Budan Theorem, there is no root of $g_{2}(x)$ in the interval $\left[2 n-4-\frac{3}{n-1},+\infty\right)$. Thus, using Lemma 1.16, we obtain that $q\left(G_{2}^{5}\right)<$ $2 n-4-\frac{3}{n-1}$.

This completes the proof.

The above two lemmas deal with the infinite classes of exceptional graphs in the statement of Theorem 2.16. For the other 18 exceptional graphs on 5-7

\begin{tabular}{|c|c|c|c|c|c|}
\hline$G$ & $\rho(G)$ & $q(G)$ & $G$ & $\rho(G)$ & $q(G)$ \\
\hline$G_{3}^{1}$ & 4.4040 & 9.3408 & $G_{3}^{16}$ & 3.2618 & 7 \\
\hline$G_{3}^{2}$ & 4.3723 & 9.3408 & $G_{3}^{17}$ & 2.3429 & 5.3234 \\
\hline$G_{3}^{4}$ & 4.2182 & 8.8965 & $G_{3}^{18}$ & 2.3028 & 4.9354 \\
\hline$G_{3}^{6}$ & 4.6056 & 9.7720 & $G_{3}^{19}$ & 2.1358 & 4.4812 \\
\hline$G_{3}^{7}$ & 3.4037 & 7.2724 & $G_{3}^{20}$ & 2.2143 & 4.6412 \\
\hline$G_{3}^{8}$ & 3.3723 & 7.4641 & $G_{3}^{21}$ & 2.6855 & 5.7785 \\
\hline$G_{3}^{9}$ & 3.6262 & 7.7588 & $G_{3}^{23}$ & 2.5616 & 5.5616 \\
\hline$G_{3}^{10}$ & 3.2814 & 7.1156 & $G_{3}^{24}$ & 2.4495 & 5 \\
\hline$G_{3}^{11}$ & 3.1413 & 6.4940 & $G_{3}^{26}$ & 3 & 6.3723 \\
\hline
\end{tabular}
vertices in the statement of Theorem 2.16, we produced the following table with the values of $\rho(G)$ and $q(G)$. These values can be easily calculated by Matlab.

TABLE 2.1 The spectral radii of some graphs

Combining all the above ingredients, we complete this chapter by presenting our short proofs of Theorem 2.6 and Theorem 2.12, respectively. 
Proof of Theorem 2.6. Suppose that $G$ is not hamiltonian. Using Lemmas 1.18 and 1.20 , we obtain

$$
\sqrt{n^{2}-4 n}<\rho(G) \leq \sqrt{2 e(G)-n+1},
$$

hence $e(G) \geq\left(\begin{array}{c}n-1 \\ 2\end{array}\right)-1$. Using Theorem 2.16, this implies $G \in\left\{G_{1}, G_{1}^{1}, G_{1}^{2}\right.$, $G_{2}^{1}, G_{2}^{2}, G_{2}^{3}, G_{2}^{4}, G_{2}^{5}, G_{3}^{1}, G_{3}^{2}, G_{3}^{4}, G_{3}^{6}, G_{3}^{7}, G_{3}^{8}, G_{3}^{9}, G_{3}^{10}, G_{3}^{11}, G_{3}^{16}, G_{3}^{17}, G_{3}^{18}, G_{3}^{19}, G_{3}^{20}$, $\left.G_{3}^{21}, G_{3}^{23}, G_{3}^{24}, G_{3}^{26}\right\}$. Since $G_{1}^{i}$ is a proper subgraph of $G_{1}(i=1,2)$, by Lemma 1.17, we have $\rho\left(G_{1}\right)>\rho\left(G_{1}^{i}\right)(i=1,2)$. Then using Lemma 2.17(i), Lemma 2.18(i), and the numerical results in Table 1 , we conclude that $G \in\left\{G_{1}, G_{1}^{1}\right.$, $\left.G_{1}^{2}, G_{3}^{6}, G_{3}^{9}, G_{3}^{17}, G_{3}^{18}, G_{3}^{21}, G_{3}^{23}, G_{3}^{24}, G_{3}^{26}\right\}$.

Proof of Theorem 2.12. Suppose that $G$ is not hamiltonian. Using Lemma 1.19 , we obtain

$$
2 n-4-\frac{3}{n-1}<q(G) \leq \frac{2 e(G)}{n-1}+n-2,
$$

hence $e(G) \geq\left(\begin{array}{c}n-1 \\ 2\end{array}\right)-1$. Using Theorem 2.16, this implies $G \in\left\{G_{1}, G_{1}^{1}, G_{1}^{2}\right.$, $G_{2}^{1}, G_{2}^{2}, G_{2}^{3}, G_{2}^{4}, G_{2}^{5}, G_{3}^{1}, G_{3}^{2}, G_{3}^{4}, G_{3}^{6}, G_{3}^{7}, G_{3}^{8}, G_{3}^{9}, G_{3}^{10}, G_{3}^{11}, G_{3}^{16}, G_{3}^{17}, G_{3}^{18}, G_{3}^{19}, G_{3}^{20}$, $\left.G_{3}^{21}, G_{3}^{23}, G_{3}^{24}, G_{3}^{26}\right\}$. Since $G_{1}^{i}$ is a proper subgraph of $G_{1}(i=1,2)$, by Lemma 1.17, we have $q\left(G_{1}\right)>q\left(G_{1}^{i}\right)(i=1,2)$. By Lemma 2.17(ii), it is possible that $G \in\left\{G_{1}, G_{1}^{1}, G_{1}^{2}\right\}$. Assuming $G \in\left\{G_{2}^{1}, G_{2}^{2}, G_{2}^{3}, G_{2}^{4}, G_{2}^{5}\right\}$, using Lemma 2.18(ii) we obtain a contradiction when $n \geq 6$. Assuming $G \in\left\{G_{3}^{1}, G_{3}^{2}, G_{3}^{4}, G_{3}^{6}, G_{3}^{7}, G_{3}^{8}\right.$, $\left.G_{3}^{9}, G_{3}^{10}, G_{3}^{11}, G_{3}^{16}, G_{3}^{17}, G_{3}^{18}, G_{3}^{19}, G_{3}^{20}, G_{3}^{21}, G_{3}^{23}, G_{3}^{24}, G_{3}^{26}\right\}$, using Table 1, we conclude that $G \in\left\{G_{3}^{6}, G_{3}^{8}, G_{3}^{9}, G_{3}^{17}, G_{3}^{21}, G_{3}^{23}, G_{3}^{26}\right\}$. 



\section{Chapter 3}

\section{Sufficient conditions for Hamilton-connectivity}

In this chapter, we aim to extend previous work about sufficient conditions for Hamilton-connectivity of graphs. We present several sufficient conditions in terms of the spectral radius or signless Laplacian spectral radius for a graph to be Hamilton-connected. The proofs of our new results involve the characterization of all the exceptional graphs.

\subsection{Introduction}

For relevant definitions and notation, we refer to the previous chapters. It is well-known that the problem of determining whether a given graph is hamiltonian, traceable or Hamilton-connected is NP-complete. Over the years, many reasonable sufficient or necessary conditions were given for the hamiltonicity or Hamilton-connectivity of graphs. We have already mentioned some of them in the former chapters. For details and other results, we refer readers to $[2,10,25,26,40-42,46,47,52,53,64-66,69]$. In this chapter, we mainly focus on giving sufficient conditions for a graph to be Hamiltonconnected. 
For edge number conditions implying Hamilton-connectedness, as we already stated in Chapter 1, Theorem 1.8 is the first result, which was given by Ore [54]. Then Zhou (the author of this thesis) and Wang extended Ore's result by relaxing the lower bound on the edge number $e(G)$. (See Theorem 1.9). Here, we prove a further strengthening of Theorem 1.9. Before stating our result, we first introduce several families of graphs.

For $n \geq 5$ and $1 \leq k \leq n / 2$, we define:

$$
S_{n}^{k}=K_{k} \vee\left(K_{n-(2 k-1)}+(k-1) K_{1}\right) \text { and } T_{n}^{k}=K_{2} \vee\left(K_{n-(k+1)}+K_{k-1}\right) \text {. }
$$

Moreover, for $t \geq 1$, let $\mathscr{S}_{n}^{k}(t)$ (resp., $\mathscr{T}_{n}^{k}(t)$ ) denote the set of all possible graphs obtained from $S_{n}^{k}$ (resp., $T_{n}^{k}$ ) by deleting exactly $t$ edges such that the minimum degree $\delta \geq 3$. Obviously, $\mathscr{S}_{n}^{k}(0)=\left\{S_{n}^{k}\right\}$ and $\mathscr{T}_{n}^{k}(0)=\left\{T_{n}^{k}\right\}$. Characterizing the exceptional graphs, we were able to prove the following result. We postpone our proof of the following results to Section 3.3.

Theorem 3.1. Let $G$ be a connected graph on $n \geq 11$ vertices and $m$ edges with $\delta(G) \geq 3$. If

$$
m \geq\left(\begin{array}{c}
n-3 \\
2
\end{array}\right)+13
$$

then $G$ is Hamilton-connected unless $G \in\left(\bigcup_{i=0}^{n-10} \mathscr{S}_{n}^{3}(i)\right) \bigcup\left(\bigcup_{i=0}^{n-11} \mathscr{T}_{n}^{3}(i)\right)$, or for $n=11, G=S_{11}^{5}$, or for $n=12, G \in \bigcup_{i=0}^{2} \mathscr{S}_{12}^{6}(i)$, or for $n=13$, $G=S_{13}^{6}$, or for $n=14, G \in \bigcup_{i=0}^{2} \mathscr{S}_{14}^{7}(i) \bigcup S_{14}^{3}(4)$, or for $n=16, G \in$ $\bigcup_{i=0}^{1} \mathscr{S}_{16}^{8}(i) \bigcup K_{7} \vee\left(K_{2}+K_{1,6}\right)$.

From Theorem 3.1, we can get the following two corollaries immediately.

Corollary 3.2. Let $G$ be a connected graph on $n \geq 14$ vertices and $m$ edges with $\delta(G) \geq 3$. If

$$
m \geq\left(\begin{array}{c}
n-2 \\
2
\end{array}\right)+4,
$$

then $G$ is Hamilton-connected unless $G \in\left(\bigcup_{i=0}^{2} \mathscr{S}_{n}^{3}(i)\right) \bigcup\left(\bigcup_{i=0}^{1} \mathscr{T}_{n}^{3}(i)\right)$, or for $n=14, G=S_{14}^{7}$. 
Corollary 3.3. Let $G$ be a connected graph on $n \geq 13$ vertices and $m$ edges with $\delta(G) \geq 3$. If

$$
m \geq\left(\begin{array}{c}
n-2 \\
2
\end{array}\right)+3
$$

then $G$ is Hamilton-connected unless $G \in\left(\bigcup_{i=0}^{3} \mathscr{S}_{n}^{3}(i)\right) \bigcup\left(\bigcup_{i=0}^{2} \mathscr{T}_{n}^{3}(i)\right)$, or for $n=13, G=S_{13}^{6}$, or for $n=14, G \in \bigcup_{i=0}^{1} \mathscr{S}_{14}^{7}(i)$.

For spectral conditions for Hamilton-connected graphs, as stated in Chapter 1, Yu and Fan [64] have established sufficient conditions on the spectral radius and signless Laplacian spectral radius for Hamilton-connectivity of graphs. Recently, Zhou and Wang [70] extended Yu and Fan's results by lowering the bound on $\rho(G)$ and $q(G)$. We recall their results here for convenience.

Theorem 3.4. (Yu and Fan [64]) Let $G$ be a graph on $n$ vertices.

(i) If $\rho(G)>-\frac{1}{2}+\sqrt{n^{2}-3 n+\frac{17}{4}}$, then $G$ is Hamilton-connected unless $G=K_{n-1}+e+e^{\prime}$.

(ii) If $\rho(\bar{G})<\sqrt{\frac{(n-2)^{2}}{n}}$, then $G$ is Hamilton-connected.

(iii) If $q(G)>2 n-4+\frac{2}{n-1}$, then $G$ is Hamilton-connected unless $G=K_{n-1}+$ $e+e^{\prime}$.

Theorem 3.5. (Zhou and Wang [70]) Let $G$ be a connected graph on $n \geq 6$ vertices with $\delta(G) \geq 3$.

(i) If $\rho(G) \geq \sqrt{n^{2}-6 n+19}$, then $G$ is Hamilton-connected.

(ii) If $q(G) \geq 2 n-6+\frac{14}{n-1}$, then $G$ is Hamilton-connected unless $G=K_{4} \vee 4 K_{1}$.

Here, we continue to study new sufficient spectral conditions for a graph to be Hamilton-connected. Our next result involves a lower bound on $\rho(G)$.

Theorem 3.6. Let $G$ be a connected graph on $n \geq 14$ vertices with $\delta(G) \geq 3$. If $\rho(G)>n-3$, then $G$ is Hamilton-connected unless $G \in\left\{S_{n}^{3}, T_{n}^{3}\right\}$. 
It is clear that the lower bound on $\rho(G)$ in Theorem 3.6 is smaller than that of Theorem 3.5. Hence Theorem 3.6 extends Theorem 3.5 in that sense. Furthermore, it is easy to see that if we perform one Kelmans operation on $T_{n}^{3}$, then we can obtain a proper subgraph of $S_{n}^{3}$. Hence by Lemma 1.17, we get $\rho\left(S_{n}^{3}\right)>\rho\left(T_{n}^{3}\right)>\rho\left(K_{n-2}\right)=n-3$. Then, using Theorem 3.6, we obtain the following corollary.

Corollary 3.7. Let $G$ be a connected graph on $n \geq 14$ vertices with $\delta(G) \geq 3$. If $\rho(G) \geq \rho\left(S_{n}^{3}\right)$, then $G$ is Hamilton-connected unless $G=S_{n}^{3}$.

Our next main result in this section is the following counterpart of Theorem 3.6.

Theorem 3.8. Let $G$ be a connected graph on $n \geq 13$ vertices with $\delta(G) \geq 3$. If $q(G)>2 n-6+\frac{6}{n-1}$, then $G$ is Hamilton-connected unless $G=S_{n}^{3}$.

Obviously, we obtain the following corollary.

Corollary 3.9. Let $G$ be a connected graph on $n \geq 13$ vertices with $\delta(G) \geq 3$. If $q(G) \geq q\left(S_{n}^{3}\right)$, then $G$ is Hamilton-connected unless $G=S_{n}^{3}$.

Let $\mathscr{G}_{n}$ be the class of non-Hamilton-connected graphs of order $n$ with $\delta \geq 3$. In Corollaries 3.7 and 3.9 , we determined the maximum spectral radius and the maximum signless Laplacian spectral radius in $\mathscr{G}_{n}$. Moreover, the extremal graphs with maximum spectral radius and maximum signless Laplacian spectral radius were also determined.

The rest of this chapter is organized as follows. In Section 3.2, we will introduce a useful lemma for Hamilton-connected graphs involving a degree sequence condition. In Section 3.3, we present the proofs of our main results.

\subsection{A useful lemma}

Some useful techniques and lemmas are already listed in Chapter 1. Here, we present an additional well-known degree sequence result for Hamiltonconnected graphs. For later reference in the proofs, we say that a sequence of non-decreasing integers is graphical if there exists a graph with that degree sequence. 
Lemma 3.10. (See, e.g., $[3,39]$ ) Let $G$ be a graph on $n \geq 3$ vertices with degree sequence $\left(d_{1}, d_{2}, \ldots, d_{n}\right)$, where $d_{1} \leq d_{2} \leq \cdots \leq d_{n}$. If there is no integer $2 \leq k \leq \frac{n}{2}$ such that $d_{k-1} \leq k$ and $d_{n-k} \leq n-k$, then $G$ is Hamilton-connected.

In the next section, we will present our proofs of the main results we listed in the previous section. For convenience, we use $\left(0^{x_{0}}, 1^{x_{1}}, \ldots, k^{x_{k}}, \ldots\right.$, $\Delta^{x_{\Delta}}$ ) to denote the degree sequence of $G$, where $x_{k}$ is the number of vertices of degree $k$ in $G$. Note that $\Delta=\Delta(G)$ denotes the maximum degree of $G$.

\subsection{Proofs of the main results}

The proof of Theorem 3.1. Suppose by contradiction that $G \notin\left(\bigcup_{i=0}^{n-10} \mathscr{S}_{n}^{3}(i)\right)$ $\bigcup\left(\bigcup_{i=0}^{n-11} \mathscr{T}_{n}^{3}(i)\right)$, and for $n=11, G \neq S_{11}^{5}$, and for $n=12, G \notin \bigcup_{i=0}^{2} \mathscr{S}_{12}^{6}(i)$, and for $n=13, G \neq S_{13}^{6}$, and for $n=14, G \notin \bigcup_{i=0}^{2} \mathscr{S}_{14}^{7}(i) \cup S_{14}^{3}(4)$, and for $n=16, G \notin \bigcup_{i=0}^{1} \mathscr{S}_{16}^{8}(i) \bigcup K_{7} \vee\left(K_{2}+K_{1,6}\right)$, and assume that $G$ is nonHamilton-connected. Suppose that $G$ has degree sequence $\left(d_{1}, d_{2}, \ldots, d_{n}\right)$, where $d_{1} \leq d_{2} \leq \cdots \leq d_{n}$. By Lemma 3.10, there exists an integer $k \leq n / 2$ such that $d_{k-1} \leq k$ and $d_{n-k} \leq n-k$. For convenience of later usage, we refer to the latter condition as the NHC-condition. Using this, we have

$$
\begin{aligned}
m & =\frac{1}{2} \sum_{i=1}^{n} d_{i} \\
& =\frac{1}{2}\left(\sum_{i=1}^{k-1} d_{i}+\sum_{i=k}^{n-k} d_{i}+\sum_{i=n-k+1}^{n} d_{i}\right) \\
& \leq \frac{1}{2}(k(k-1)+(n-k)(n-2 k+1)+(n-1) k) \\
& =\left(\begin{array}{c}
n-3 \\
2
\end{array}\right)+13+\frac{f(k)}{2},
\end{aligned}
$$

where $f(x):=3 x^{2}-(2 n+3) x+8 n-38$. Since $e(G) \geq\left(\begin{array}{c}n-3 \\ 2\end{array}\right)+13$ by assumption, combining this with (3.1), we have $f(k) \geq 0$. Moreover, noting that $3 \leq \delta \leq d_{k-1} \leq k \leq n / 2$, by a direct computation we obtain:

- for $n \geq 11$, we have $f(3)=2 n-20>0, f(4)=-2<0$. 
Next, we calculate $f(k)$ for $k \geq 5$. We have:

- if $n=11$, then $k \leq 5$ and $f(5)=0$;

- if $n=12$, then $k \leq 6$ and $f(5)=-2<0, f(6)=4>0$;

- if $n=13$, then $k \leq 6$ and $f(5)=-4<0, f(6)=0$;

- if $n=14$, then $k \leq 7$ and $f(5)=-6<0, f(6)=-4<0, f(7)=4>0$;

- if $n=15$, then $k \leq 7$ and $f(5)=-8<0, f(6)=-8<0, f(7)=-2<$ 0 ;

- if $n=16$, then $k \leq 8$ and $f(5)=-10<0, f(6)=-12<0, f(7)=$ $-8<0, f(8)=2>0$;

- if $n=17$, then $k \leq 8$ and $f(5)=-12<0, f(6)=-16<0, f(7)=$ $-14<0, f(8)=-6<0$;

- if $n \geq 18$, then for $5 \leq k \leq n / 2$, we have $f(k)<0$. To see this, we consider the two roots of $f(x)=0$, which are

$$
\begin{aligned}
& r_{1}=\frac{2 n+3-\sqrt{(2 n+3)^{2}-12(8 n-38)}}{6}, \\
& r_{2}=\frac{2 n+3+\sqrt{(2 n+3)^{2}-12(8 n-38)}}{6} .
\end{aligned}
$$

By simple calculations, we see that both $r_{1}<5$ and $r_{2}>n / 2$ hold for $n \geq 18$, and then the desired result follows.

Using the above computational results, we discuss the following cases.

Case 1. $k=3$ and $n \geq 11$.

In this case, we shall show that by our assumption that $G$ is not Hamiltonconnected, it follows that $G \in\left(\bigcup_{i=0}^{n-10} \mathscr{S}_{n}^{3}(i)\right) \bigcup\left(\bigcup_{i=0}^{n-11} \mathscr{T}_{n}^{3}(i)\right)$, which is a contradiction to our other assumption.

By the NHC-condition, we have

$$
d_{1}=d_{2}=3, d_{3} \leq \cdots \leq d_{n-3} \leq n-3, d_{n-2} \leq d_{n-1} \leq d_{n} \leq n-1 .
$$


Furthermore, noting that $f(3)=2 n-20$ when $n \geq 11$, by (3.1), we have

$$
\begin{gathered}
\left(\begin{array}{c}
n-3 \\
2
\end{array}\right)+13 \leq e(G) \leq\left(\begin{array}{c}
n-3 \\
2
\end{array}\right)+13+(n-10) . \\
\text { If } e(G)=\left(\begin{array}{c}
n-3 \\
2
\end{array}\right)+13+(n-10) \text {, then } \sum_{i=1}^{n} d_{i}=2 e(G)=n^{2}-5 n+18 .
\end{gathered}
$$

Hence it follows that the inequalities in (3.2) must be equalities, and that the degree sequence of $G$ is $(3,3, \underbrace{n-3, \ldots, n-3}_{n-5 \text { times }}, n-1, n-1, n-1)$, which implies that $G=S_{n}^{3}=\mathscr{S}_{n}^{3}(0)$, a contradiction. have

If $e(G)=\left(\begin{array}{c}n-3 \\ 2\end{array}\right)+13+(n-10)-t$, where $1 \leq t \leq n-10$, then we

$$
e(G)=e\left(S_{n}^{3}\right)-t \text { and } e(G)=e\left(T_{n}^{3}\right)-(t-1) .
$$

We use the shorthand $r$-degree vertex for a vertex with degree $r$. Noting that any three 3-degree vertices are incident with at most 9 edges, any $n-3$ vertices are incident with at most $\left(\begin{array}{c}n-3 \\ 2\end{array}\right)$ edges, and $e(G) \geq\left(\begin{array}{c}n-3 \\ 2\end{array}\right)+$ 13 , we conclude that $G$ has exactly two 3 -degree vertices. Without loss of generality, we may suppose that $d_{G}\left(v_{1}\right)=d_{G}\left(v_{2}\right)=3$. Next, we discuss the following two subcases.

Subcase 1.1. $v_{1}$ is not adjacent to $v_{2}$.

If $N_{G}\left(v_{1}\right)=N_{G}\left(v_{2}\right)$, then combining the definition of $S_{n}^{k}$ and (3.3), $G$ is a subgraph of $S_{n}^{3}$ obtained by deleting $t(1 \leq t \leq n-10)$ edges from its clique $K_{n-2}$. That is to say, $G \in \bigcup_{t=1}^{n-10} \mathscr{S}_{n}^{3}(t)$, which is a contradiction to our assumption.

Next, assume $N_{G}\left(v_{1}\right) \neq N_{G}\left(v_{2}\right)$. Let $H_{1}=G\left[V(G) \backslash\left\{v_{1}\right\}\right]$. Then, $\left|V\left(H_{1}\right)\right|=$ $n-1, \delta\left(H_{1}\right) \geq 3$ and $e\left(H_{1}\right)=e(G)-3 \geq\left(\begin{array}{c}n-3 \\ 2\end{array}\right)+10=\left(\begin{array}{c}(n-1)-2 \\ 2\end{array}\right)+$ $10>\left(\begin{array}{c}(n-1)-2 \\ 2\end{array}\right)+6$. Hence by Theorem 1.9, we have that $H_{1}$ is Hamiltonconnected. If every two vertices in $V\left(H_{1}\right)$ can be connected by a Hamilton path in $G$, then $G$ is also Hamilton-connected, a contradiction. Thus, there 
must exist two vertices $w$ and $w^{\prime}$ such that they are connected by a path passing through all vertices in $V(G)$ but not $v_{1}$. Let $P$ be this path in a given direction (from $w$ to $w^{\prime}$ ). Suppose the vertices in $P$ are $w=y_{1}, y_{2}, \ldots, y_{n-1}=w^{\prime}$ in sequence. Let $y_{i}, y_{j}$ and $y_{l}(1 \leq i<j<l \leq n-1)$ be three vertices adjacent to $v_{1}$. Then it is obvious that $\left\{v_{1}, y_{i+1}, y_{j+1}\right\}$ is an independent set since $G$ is not Hamilton-connected. We claim that

$$
d_{G}\left(y_{i+1}\right)+d_{G}\left(y_{j+1}\right) \leq n .
$$

To see this, consider the set $K=\left\{y_{r} \mid y_{r-1} \in N_{G}\left(y_{i+1}\right) \cap V\left(H_{1}\right), r-1 \leq i\right.$ or $r-$ $1 \geq j+2\} \bigcup\left\{y_{s} \mid y_{s+1} \in N_{G}\left(y_{i+1}\right) \cap V\left(H_{1}\right), i+1<s+1 \leq j\right\}$. Note that $|K| \geq d_{G}\left(y_{i+1}\right)-2$. This follows since the vertex $y_{i+1}$ is possibly adjacent to $y_{n-1}=w^{\prime}$ and $y_{i+1}$ is both the successor of $y_{i}$ and the predecessor of $y_{i+2}$. Since $\left\{v_{1}, y_{i+1}, y_{j+1}\right\}$ is an independent set, we obtain $d_{H_{1}}\left(y_{i+1}\right)=d_{G}\left(y_{i+1}\right)$, $d_{H_{1}}\left(y_{j+1}\right)=d_{G}\left(y_{j+1}\right)$, and $\left|K \cup N_{G}\left(y_{j+1}\right)\right| \leq\left|V\left(H_{1}\right)\right|-\left|\left\{y_{j+1}\right\}\right|=n-2$. Thus, if $d_{G}\left(y_{i+1}\right)+d_{G}\left(y_{j+1}\right) \geq n+1$, then

$$
\begin{aligned}
\left|K \cap N_{G}\left(y_{j+1}\right)\right| & =|K|+\left|N_{G}\left(y_{j+1}\right)\right|-\left|K \cup N_{G}\left(y_{j+1}\right)\right| \\
& \geq d_{G}\left(y_{i+1}\right)-2+d_{G}\left(y_{j+1}\right)-(n-2) \\
& \geq n+1-2-(n-2)=1,
\end{aligned}
$$

implying that $K$ and $N_{G}\left(y_{j+1}\right)$ have a common vertex, say $y_{t}$. Obviously, $t \neq i+1, j+1$. If $t=i$, then $y_{i+1} y_{j+1} \in E(G)$, a contradiction. If $t \leq$ $i-1$, then $y_{i+1} y_{t-1} \in E(G)$. Then $y_{1} \vec{P} y_{t-1} y_{i+1} \vec{P} y_{j} v_{1} y_{i} \overleftarrow{P} y_{t} y_{j+1} \vec{P} y_{n-1}$ is a Hamilton path in $G$ connecting $y_{1}$ and $y_{n-1}$, a contradiction. If $t \geq$

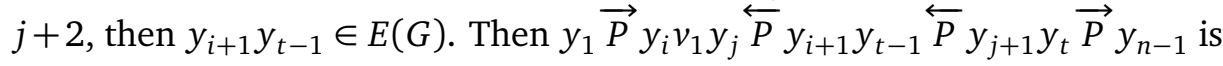
a Hamilton path in $G$ connecting $y_{1}$ and $y_{n-1}$, a contradiction. If $i+1<t \leq$ $j$, then $y_{i+1} y_{t+1} \in E(G)$. Then $y_{1} \vec{P} y_{i} v_{1} y_{j} \stackrel{\leftarrow}{P} y_{t+1} y_{i+1} \vec{P} y_{t} y_{j+1} \vec{P} y_{n-1}$ is a Hamilton path in $G$ connecting $y_{1}$ and $y_{n-1}$, a contradiction. Hence (3.4) holds. Next, according to the distribution of the neighbors of $v_{1}$, we discuss the following three subcases.

Subcase 1.1.1. We assume $y_{i+1} \neq y_{j-1}$ and $y_{j+1} \neq y_{l-1}$.

Then by a similar method as above, we obtain $d_{G}\left(y_{j-1}\right)+d_{G}\left(y_{l-1}\right) \leq n$. Consequently, by considering the number of edges in $G$, we can get the following 
contradiction:

$$
\begin{aligned}
e(G) \leq & 3+\left(d_{G}\left(y_{i+1}\right)+d_{G}\left(y_{j+1}\right)\right)+\left(d_{G}\left(y_{j-1}\right)+d_{G}\left(y_{l-1}\right)\right) \\
& +e\left(G\left[V(G) \backslash\left\{v_{1}, y_{i+1}, y_{j+1}, y_{j-1}, y_{l-1}\right\}\right]\right) \\
\leq & 3+n+n+\left(\begin{array}{c}
n-5 \\
2
\end{array}\right)=\left(\begin{array}{c}
n-3 \\
2
\end{array}\right)+12<\left(\begin{array}{c}
n-3 \\
2
\end{array}\right)+13 \\
\leq & e(G),
\end{aligned}
$$

which is a contradiction. Here the first inequality follows from the observation that there may be edges joining vertices in the set $\left\{y_{i+1}, y_{j+1}, y_{j-1}, y_{l-1}\right\}$.

Subcase 1.1.2. We assume $y_{i+1}=y_{j-1}$ and $y_{j+1} \neq y_{l-1}$.

Then $y_{j}=y_{i+2}$ and $y_{j+1}=y_{i+3}$. If $y_{l} \neq y_{n-1}$, then by using a similar method as in Subcase 1.1.1, we can obtain

$$
\begin{aligned}
e(G) \leq & 3+\left(d_{G}\left(y_{i+1}\right)+d_{G}\left(y_{l-1}\right)\right)+\left(d_{G}\left(y_{i+3}\right)+d_{G}\left(y_{l+1}\right)\right) \\
& +e\left(G\left[V(G) \backslash\left\{v_{1}, y_{i+1}, y_{l-1}, y_{i+3}, y_{l+1}\right\}\right]\right) \\
\leq & 3+n+n+\left(\begin{array}{c}
n-5 \\
2
\end{array}\right)=\left(\begin{array}{c}
n-3 \\
2
\end{array}\right)+12<\left(\begin{array}{c}
n-3 \\
2
\end{array}\right)+13 \\
\leq & e(G),
\end{aligned}
$$

which is a contradiction. Here the first inequality follows from the observation that there may be edges joining vertices in the set $\left\{y_{i+1}, y_{l-1}, y_{i+3}, y_{l+1}\right\}$.

Next, we suppose $y_{l}=y_{n-1}$. If $y_{i} \neq y_{1}$, then by using a similar method as in Subcase 1.1.1, we can obtain

$$
\begin{aligned}
e(G) \leq & 3+\left(d_{G}\left(y_{i-1}\right)+d_{G}\left(y_{l-1}\right)\right)+\left(d_{G}\left(y_{i+1}\right)+d_{G}\left(y_{i+3}\right)\right) \\
& +e\left(G\left[V(G) \backslash\left\{v_{1}, y_{i-1}, y_{l-1}, y_{i+1}, y_{i+3}\right\}\right]\right) \\
\leq & 3+n+n+\left(\begin{array}{c}
n-5 \\
2
\end{array}\right)=\left(\begin{array}{c}
n-3 \\
2
\end{array}\right)+12<\left(\begin{array}{c}
n-3 \\
2
\end{array}\right)+13 \\
\leq & e(G),
\end{aligned}
$$

which is a contradiction. Here the first inequality follows from the observation that there may be edges joining vertices in the set $\left\{y_{i-1}, y_{l-1}, y_{i+1}, y_{i+3}\right\}$. 
If $y_{i}=y_{1}$, then $v_{1}$ is adjacent to $y_{1}, y_{3}$ and $y_{n-1}$. Let $W_{1}=V(G) \backslash$ $\left\{v_{1}, y_{1}, y_{3}\right\}$. Then we show $\delta\left(G\left[W_{1}\right]\right) \geq 3$.

If $d_{G\left[W_{1}\right]}\left(y_{2}\right)=1$, then $y_{2}=v_{2}$. Since $N_{G}\left(v_{1}\right) \neq N_{G}\left(v_{2}\right)$ and $\left\{v_{1}, y_{2}, y_{4}\right\}$ is an independent set, $W_{1} \backslash\left\{y_{4}, y_{n-1}\right\}$ has one vertex $w_{1}$ such that $y_{2} w_{1} \in E(G)$. Let $W_{2}=W_{1} \backslash\left\{y_{2}\right\}$. Then

$$
\begin{aligned}
e\left(G\left[W_{2}\right]\right) \geq & \left(\begin{array}{c}
n-3 \\
2
\end{array}\right)+13-3-d_{G}\left(y_{2}\right) \\
& -d_{G\left[W_{2} \cup\left\{y_{1}, y_{3}\right\}\right]}\left(y_{1}\right)-d_{G\left[W_{2} \cup\left\{y_{3}\right\}\right]}\left(y_{3}\right) \\
\geq & \left(\begin{array}{c}
n-3 \\
2
\end{array}\right)+13-3-3-(n-3)-(n-4) \\
= & \left(\begin{array}{c}
(n-4)-1 \\
2
\end{array}\right)+5,
\end{aligned}
$$

which, together with the fact that $\left|W_{2}\right|=n-4$ and Theorem 1.8, implies that $G\left[W_{2}\right]$ is Hamilton-connected. Then there is a Hamilton path $w_{1} P y_{n-1}$ which connects $w_{1}$ and $y_{n-1}$ in $G\left[W_{2}\right]$. Then $y_{1} v_{1} y_{3} y_{2} w_{1} P y_{n-1}$ is a Hamilton path connecting $y_{1}$ and $y_{n-1}$ in $G$, a contradiction.

If $d_{W_{1}}\left(y_{2}\right)=2$, then $d_{G}\left(y_{2}\right)=4$. There always exists a vertex $w_{1}$ as above. Then

$$
\begin{aligned}
e\left(G\left[W_{2}\right]\right) \geq & \left(\begin{array}{c}
n-3 \\
2
\end{array}\right)+13-3-d_{G}\left(y_{2}\right) \\
& -d_{G\left[W_{2} \cup\left\{y_{1}, y_{3}\right\}\right]}\left(y_{1}\right)-d_{G\left[W_{2} \cup\left\{y_{3}\right\}\right]}\left(y_{3}\right) \\
\geq & \left(\begin{array}{c}
n-3 \\
2
\end{array}\right)+13-3-4-(n-3)-(n-4) \\
= & \left(\begin{array}{c}
(n-4)-1 \\
2
\end{array}\right)+4 .
\end{aligned}
$$

Hence we can also get a contradiction by a similar method as before.

If $d_{W_{1}}\left(y_{4}\right)=1$ or 2 , then $d_{G}\left(y_{4}\right)=3$ or 4 . Let $W_{3}=W_{1} \backslash\left\{y_{4}\right\}$. By a similar discussion as above, we can obtain that $G\left[W_{3}\right]$ is Hamilton-connected, and we also derive a contradiction. 
If there exist a vertex $y_{k} \in W_{1} \backslash\left\{y_{2}, y_{4}\right\}$ which satisfies that $d_{G\left[W_{1}\right]}\left(y_{k}\right) \leq$ 2 , then $d_{G}\left(y_{k}\right) \leq 4$. Since $d_{G}\left(y_{2}\right)+d_{G}\left(y_{4}\right) \leq n$,

$$
\begin{aligned}
e(G) & \leq d_{G}\left(v_{1}\right)+d_{G}\left(y_{k}\right)+\left(d_{G}\left(y_{2}\right)+d_{G}\left(y_{4}\right)\right)+e\left(G\left[V(G) \backslash\left\{v_{1}, y_{2}, y_{4}, y_{k}\right\}\right]\right) \\
& \leq 3+4+n+\left(\begin{array}{c}
n-4 \\
2
\end{array}\right)=\left(\begin{array}{c}
n-3 \\
2
\end{array}\right)+11<\left(\begin{array}{c}
n-3 \\
2
\end{array}\right)+13,
\end{aligned}
$$

which is a contradiction.

Hence $\delta\left(G\left[W_{1}\right]\right) \geq 3$. Note that $\left|W_{1}\right|=n-3$, and

$$
\begin{aligned}
e\left(G\left[W_{1}\right]\right) & \geq\left(\begin{array}{c}
n-3 \\
2
\end{array}\right)+13-3-d_{G\left[W_{1} \cup\left\{y_{1}, y_{3}\right\}\right]}\left(y_{1}\right)-d_{G\left[W_{1} \cup\left\{y_{3}\right\}\right]}\left(y_{3}\right) \\
& \geq\left(\begin{array}{c}
n-3 \\
2
\end{array}\right)+13-3-(n-2)-(n-3)=\left(\begin{array}{c}
(n-3)-2 \\
2
\end{array}\right)+6 .
\end{aligned}
$$

Then by Theorem 1.9, we have that $G\left[W_{1}\right]$ is either Hamilton-connected, or $G\left[W_{1}\right]=K_{3} \vee\left(K_{(n-3)-5}+2 K_{1}\right)$. If $G\left[W_{1}\right]$ is Hamilton-connected, then there is a Hamilton path connecting $y_{2}$ and $y_{n-1}$ in $G\left[W_{1}\right]$, say $y_{2} P y_{n-1}$. Then $y_{1} v_{1} y_{3} y_{2} P y_{n-1}$ is a Hamilton path connecting $y_{1}$ and $y_{n-1}$ in $G$, a contradiction. If $G\left[W_{1}\right]=K_{3} \vee\left(K_{(n-3)-5}+2 K_{1}\right)$, then $e\left(G\left[W_{1}\right]\right)=\left(\begin{array}{c}(n-3)-2 \\ 2\end{array}\right)+6$, $d_{G\left[W_{1} \cup\left\{y_{1}, y_{3}\right\}\right]}\left(y_{1}\right)=n-2$ and $d_{G\left[W_{1} \cup\left\{y_{3}\right\}\right]}\left(y_{3}\right)=n-3$. Therefore, we have $d_{G}\left(y_{1}\right)=d_{G}\left(y_{3}\right)=n-1$ and $G$ has only one 3-degree vertex $v_{1}$, which contradicts the fact that $G$ has exactly two 3-degree vertices.

Furthermore, the case that $y_{i+1} \neq y_{j-1}$ and $y_{j+1}=y_{l-1}$ can be treated in a similar way, hence we omit it.

Subcase 1.1.3. We assume $y_{i+1}=y_{j-1}$ and $y_{j+1}=y_{l-1}$.

Then $y_{j}=y_{i+2}$ and $y_{l}=y_{i+4}$. If $v_{1}$ is adjacent to neither $y_{1}$ nor $y_{n-1}$, then there must exist $y_{i-1}$ and $y_{i+5}$ since $n \geq 11$. Then by using a similar method as in Subcase 1.1.1, we can obtain

$$
\begin{aligned}
e(G) \leq & 3+\left(d_{G}\left(y_{i-1}\right)+d_{G}\left(y_{i+3}\right)\right)+\left(d_{G}\left(y_{i+1}\right)+d_{G}\left(y_{i+5}\right)\right) \\
& +e\left(G\left[V(G) \backslash\left\{v_{1}, y_{i-1}, y_{i+3}, y_{i+1}, y_{i+5}\right\}\right]\right)
\end{aligned}
$$




$$
\begin{aligned}
& \leq 3+n+n+\left(\begin{array}{c}
n-5 \\
2
\end{array}\right)=\left(\begin{array}{c}
n-3 \\
2
\end{array}\right)+12<\left(\begin{array}{c}
n-3 \\
2
\end{array}\right)+13 \\
& \leq e(G),
\end{aligned}
$$

which is a contradiction. Here the first inequality follows from the observation that there may be edges joining vertices in the set $\left\{y_{i-1}, y_{i+3}, y_{i+1}, y_{i+5}\right\}$.

If $v_{1}$ is adjacent to $y_{1}$, then $v_{1}$ is also adjacent to $y_{3}$ and $y_{5}$. One may easily get a contradiction by a similar discussion as in Subcase 1.1.2.

Similarly, if $v_{1}$ is adjacent to $y_{n}$, then $v_{1}$ is also adjacent to $y_{n-3}$ and $y_{n-5}$. One can also get a contradiction by a similar discussion as in Subcase 1.1.2.

Subcase 1.2. $v_{1}$ is adjacent to $v_{2}$.

Consider the graph $H_{2}:=G\left[V(G) \backslash\left\{v_{1}, v_{2}\right\}\right]$. It is not difficult to see that $\left|V\left(H_{2}\right)\right|=n-2$ and $e\left(H_{2}\right)=e(G)-5 \geq\left(\begin{array}{c}(n-2)-1 \\ 2\end{array}\right)+8$. Then by Theorem 1.8, we get that $H_{2}$ is Hamilton-connected. There must exist two vertices $w$ and $w^{\prime}$ such that they are connected by a path passing through all vertices in $V\left(H_{2}\right)$ but not through $v_{1}$ and $v_{2}$ at the same time. We denote this path by $y_{1} P^{\prime} y_{n-2}$, where $y_{1}=w, y_{n-2}=w^{\prime}$, and give this path a direction (from $w$ to $w^{\prime}$ ). If $u$ is on this path, we use $u^{+}$and $u^{-}$to denote the successor and predecessor of $u$, respectively. Since $d_{G}\left(v_{1}\right)=d_{G}\left(v_{2}\right)=3$, there must be two vertices of $H_{2}$, say $z_{1}$ and $z_{2}$ (they are in this order on this path), which are adjacent to $v_{1}$. Also, there must be two vertices $z_{3}$ and $z_{4}$ (they are in this order on this path) of $H_{2}$ which are adjacent to $v_{2}$.

We now claim that $z_{1}=z_{3}$ and $z_{2}=z_{4}$, which, together with (3.3), would yield that $G$ is a subgraph of $T_{n}^{3}$ obtained by deleting $t-1$ edges from its clique $K_{n-2}$, that is, $G \in \mathscr{T}_{n}^{3}(t-1)$, where $1 \leq t \leq n-10$.

Suppose to the contrary that $z_{1} \neq z_{3}$ or $z_{2} \neq z_{4}$. We can easily see that $z_{i} \neq z_{1}^{-}, z_{1}^{+}, z_{2}^{-}, z_{2}^{+}(i=3,4), z_{j} \neq z_{3}^{-}, z_{3}^{+}, z_{4}^{-}, z_{4}^{+}(j=1,2)$. And, if $z_{2}=z_{1}^{+}$, then $z_{4} \neq z_{3}^{+}$and vice versa. 
If $z_{1} \neq z_{3}$ and $z_{2} \neq z_{4}$, then by the same discussion as we used for (3.4), we have $d_{G}\left(z_{1}^{+}\right)+d_{G}\left(z_{3}^{+}\right) \leq n-1$. Then,

$$
\begin{aligned}
e(G) & =5+\left(d_{G}\left(z_{1}^{+}\right)+d_{G}\left(z_{3}^{+}\right)\right)+e\left(G\left[V(G) \backslash\left\{v_{1}, v_{2}, z_{1}^{+}, z_{3}^{+}\right\}\right]\right) \\
& \leq 5+n-1+\left(\begin{array}{c}
n-4 \\
2
\end{array}\right)=\left(\begin{array}{c}
n-3 \\
2
\end{array}\right)+8<\left(\begin{array}{c}
n-3 \\
2
\end{array}\right)+13 \\
& \leq e(G),
\end{aligned}
$$

which is a contradiction.

If $z_{1}=z_{3}$ and $z_{2} \neq z_{4}$, then by the same discussion as we used for (3.4), we have $d_{G}\left(z_{2}^{-}\right)+d_{G}\left(z_{4}^{-}\right) \leq n-1$. Then,

$$
\begin{aligned}
e(G) & =5+\left(d_{G}\left(z_{2}^{-}\right)+d_{G}\left(z_{4}^{-}\right)\right)+e\left(G\left[V(G) \backslash\left\{v_{1}, v_{2}, z_{2}^{-}, z_{4}^{-}\right\}\right]\right) \\
& \leq 5+n-1+\left(\begin{array}{c}
n-4 \\
2
\end{array}\right)=\left(\begin{array}{c}
n-3 \\
2
\end{array}\right)+8<\left(\begin{array}{c}
n-3 \\
2
\end{array}\right)+13 \\
& \leq e(G),
\end{aligned}
$$

which is a contradiction.

The case that $z_{1} \neq z_{3}$ and $z_{2}=z_{4}$ can be discussed in a similar way.

Summing up the above discussions, we have $G \in\left(\bigcup_{i=0}^{n-10} \mathscr{S}_{n}^{3}(i)\right) \bigcup\left(\bigcup_{i=0}^{n-11}\right.$ $\mathscr{T}_{n}^{3}(i)$ ), as desired.

Case 2. $n=11$ and $k=5$.

In this case, by the NHC-condition we have

$$
d_{1} \leq d_{2} \leq d_{3} \leq d_{4} \leq 5, d_{5} \leq d_{6} \leq 6, d_{7} \leq \cdots \leq d_{11} \leq 10 .
$$

Moreover, since $f(5)=0$ when $n=11$, we obtain $e(G)=\left(\begin{array}{c}n-3 \\ 2\end{array}\right)+13=41$, and then $\sum_{i=1}^{11} d_{i}=82$. Combining this with (3.5), we conclude that the degree sequence of $G$ is $\left(5^{4}, 6^{2}, 10^{5}\right)$, which implies $G=K_{5} \vee\left(K_{2}+4 K_{1}\right)=S_{11}^{5}$, a contradiction.

Case 3. $n=12$ and $k=6$. 
Again, by the NHC-condition we have

$$
d_{1} \leq d_{2} \leq d_{3} \leq d_{4} \leq d_{5} \leq 6, d_{6} \leq 6, d_{7} \leq \cdots \leq d_{12} \leq 11 .
$$

Noting that $f(6)=4$ when $n=12$, and by (3.1), we have $49 \leq e(G) \leq 51$. If $e(G)=51$, then $\sum_{i=1}^{12} d_{i}=102$, which, together with (3.6), yields that the degree sequence of $G$ is $\left(6^{6}, 11^{6}\right)$. From this one can check directly that $G=K_{6} \vee 6 K_{1}=S_{12}^{6}=\mathscr{S}_{12}^{6}(0)$.

Now assume that

$$
e(G)=51-t=e\left(S_{12}^{6}\right)-t, \text { where } t \in\{1,2\} .
$$

Since $\sum_{i=1}^{12} d_{i}=102-2 t \geq 98, G$ has at least one 6-degree vertex and has no 3-degree vertex. Let $d_{G}\left(x_{0}\right)=6$ and $H_{3}:=G\left[V(G) \backslash\left\{x_{0}\right\}\right]$. It is easy to see that $\left|V\left(H_{3}\right)\right|=11, \delta\left(H_{3}\right) \geq 3$ and $e\left(H_{3}\right)=e(G)-6 \geq 49-$ $6=43>\left(\begin{array}{c}11-2 \\ 2\end{array}\right)+6$, so by Theorem 1.9, we get that $H_{3}$ is Hamiltonconnected. Let $w P w^{\prime}$ be a Hamilton path in $H_{3}$ from $w$ to $w^{\prime}$. Since $G$ is not Hamilton-connected, there is no Hamilton path connecting $w$ and $w^{\prime}$ in $G$. Suppose that $x_{1}, x_{2}, x_{3}, x_{4}, x_{5}, x_{6}$ are distinct vertices of $H_{3}$ which are adjacent to $x_{0}$. Without loss of generality, we assume that $x_{6}=w^{\prime}$. Then $\left\{x_{0}, x_{1}^{+}, x_{2}^{+}, x_{3}^{+}, x_{4}^{+}, x_{5}^{+}\right\}$is an independent set, which, together with (3.7), would yield that $G$ is a subgraph of $S_{12}^{6}$ obtained by deleting any $t$ edges, that is, $G \in \mathscr{S}_{12}^{6}(t)$, where $t \in\{1,2\}$.

Summing up the above discussions, we eventually obtain $G \in \bigcup_{i=0}^{2} \mathscr{S}_{12}^{6}(i)$, a contradiction.

Case 4. $n=13$ and $k=6$.

This case is completely analogous to Case 2 . We can obtain $e(G)=58$, $\sum_{i=1}^{13} d_{i}=116$, and the degree sequence of $G$ is $\left(6^{5}, 7^{2}, 12^{6}\right)$, which implies that $G=K_{6} \vee\left(K_{2}+5 K_{1}\right)=S_{13}^{6}$, a contradiction.

Case 5. $n=14$ and $k=7$.

As in Case 3 , we have $d_{1} \leq \cdots \leq d_{6} \leq 7, d_{7} \leq 7, d_{8} \leq \cdots \leq d_{14} \leq 13$ and $68 \leq e(G) \leq 70$. Since $\sum_{i=1}^{14} d_{i} \geq 136, G$ has at least one 7-degree vertex and has no 3-degree vertex. Let $d_{G}\left(x_{0}\right)=7$ and $H_{4}=G\left[V(G) \backslash\left\{x_{0}\right\}\right]$. Obviously, 
$\left|V\left(H_{4}\right)\right|=13$ and $\delta\left(H_{4}\right) \geq 3$.

If $e(G)=70$, then the degree sequence of $G$ must be $\left(7^{7}, 13^{7}\right)$, which implies that $G=K_{7} \vee 7 K_{1}=S_{14}^{7}=\mathscr{S}_{14}^{7}(0)$.

If $e(G)=70-1=69$, then $e\left(H_{4}\right)=e(G)-7=62>\left(\begin{array}{c}13-2 \\ 2\end{array}\right)+6=61$, so by Theorem 1.9, $\mathrm{H}_{4}$ is Hamilton-connected. Hence, by similar arguments as in Case 3, we would get $G \in \mathscr{S}_{14}^{7}(1)$.

If $e(G)=70-2=68$, then $e\left(H_{4}\right)=e(G)-7=61=\left(\begin{array}{c}13-2 \\ 2\end{array}\right)+6$, so by Theorem 1.9, $H_{4}$ is either Hamilton-connected or $H_{4}=K_{3} \vee\left(K_{8}+2 K_{1}\right)$. If $\mathrm{H}_{4}$ is Hamilton-connected, then by similar arguments as in Case 3, we would get $G \in \mathscr{S}_{14}^{7}(2)$, a contradiction. Hence, $H_{4}=K_{3} \vee\left(K_{8}+2 K_{1}\right)=S_{13}^{3}$. In this case, if $x_{0}$ is not adjacent to the two 3-degree vertices of $H_{4}$, then it is evident that $G$ is a subgraph of $S_{14}^{3}$ with $e\left(S_{14}^{3}\right)-4$ edges, that is, $G \in \mathscr{S}_{14}^{3}(4)$; otherwise, one may check easily that $G$ is Hamilton-connected, contradicting our assumption.

Summing up the above discussions, we eventually get $G \in \bigcup_{i=0}^{2} \mathscr{S}_{14}^{7}(i) \bigcup$ $\mathscr{S}_{14}^{3}(4)$.

Case 6. $n=16$ and $k=8$.

Similarly, we have $d_{1} \leq \cdots \leq d_{7} \leq 8, d_{8} \leq 8, d_{9} \leq \cdots \leq d_{16} \leq 15,91 \leq$ $e(G) \leq 92$, and hence $182 \leq \sum_{i=1}^{16} d_{i} \leq 184$. From the inequality $\sum_{i=9}^{16} d_{i}=$ $2 m-\sum_{i=1}^{8} d_{i} \geq 182-64=118$, we get that $d_{11}=\cdots=d_{16}=15$ and $d_{9}+d_{10} \geq 28$. Note that $\sum d_{i}$ is even and the total degree sum is between 182 and 184. If $d_{9}=d_{10}=15$, then the permissible graphical sequence is $\left(8^{8}, 15^{8}\right)$, which implies that $G=K_{8} \vee 8 K_{1}=S_{16}^{8} \in \mathscr{S}_{16}^{8}(0)$. If $d_{9}=14$ and $d_{10}=15$, then the permissible graphical sequence is $\left(7^{1}, 8^{7}, 14^{1}, 15^{7}\right)$, which implies $G=K_{7} \vee\left(K_{1}+K_{1,7}\right)$. If $d_{9}=13$ and $d_{10}=15$, then the permissible graphical sequence is $\left(8^{8}, 13^{1}, 15^{7}\right)$, which implies that $G=K_{7} \vee\left(K_{2}+K_{1,6}\right)$. If $d_{9}=d_{10}=14$, then the permissible graphical sequence is $\left(8^{8}, 14^{2}, 15^{6}\right)$. If $v_{9}$ is adjacent to $v_{10}$, then $G$ can be obtained as follows. Let $X=K_{8}$, $Y=8 K_{1}, x_{1}, x_{2} \in V(X)$ and $y_{1}, y_{2} \in V(Y) . G$ is a subgraph of $X \vee Y$ obtained by deleting $x_{1} y_{1}, x_{2} y_{2}$ and adding a new edge $y_{1} y_{2}$. Note that in this case $G$ is Hamilton-connected. If $v_{9}$ is not adjacent to $v_{10}$, then $G=K_{6} \vee K_{2,8}$. 
Since $\mathscr{S}_{16}^{8}(1)=\left\{K_{7} \vee\left(K_{1}+K_{1,7}\right), K_{6} \vee K_{2,8}\right\}$, summing up the above discussions, we get $G \in \bigcup_{i=0}^{1} \mathscr{S}_{16}^{8}(i) \bigcup\left(K_{7} \vee\left(K_{2}+K_{1,6}\right)\right)$.

This completes the proof of Theorem 3.1.

The proof of Theorem 3.6. Suppose that $G$ is not Hamilton-connected. By Lemmas 1.18 and 1.20 , we have $\rho(G) \leq 1+\sqrt{2 m-3 n+4}$, which, together with the condition of Theorem 3.6, yields $n-3<\rho(G) \leq 1+\sqrt{2 m-3 n+4}$. We obtain $2 m>n^{2}-5 n+12$. Furthermore, using parity arguments, we obtain $m \geq\left(\begin{array}{c}n-2 \\ 2\end{array}\right)+4$. By Corollary 3.2, we have $G \in\left(\bigcup_{i=0}^{2} \mathscr{S}_{n}^{3}(i)\right) \bigcup\left(\bigcup_{i=0}^{1}\right.$ \left.${\mathscr{T}_{n}^{3}}^{3}(i)\right)$ or for $n=14, G=S_{14}^{7}$.

Noting that $K_{n-2}$ is a proper subgraph of $S_{n}^{3}$ and $T_{n}^{3}$, by Lemma 1.17, we have $\rho\left(S_{n}^{3}\right)>\rho\left(K_{n-2}\right)=n-3$ and $\rho\left(T_{n}^{3}\right)>\rho\left(K_{n-2}\right)=n-3$. So $S_{n}^{3}$ and $T_{n}^{3}$ enter the list of exceptions of the theorem. We continue to show that there are no other exceptional graphs, by analyzing and eliminating all the possible cases.

For $G \in \mathscr{S}_{n}^{3}(1)$, that is, if $G$ is obtained from the graph $S_{n}^{3}$ by removing one edge, we have to check the following possible degree sequences for graphs $G=H_{i}$ :

1. $H_{1}$ has degree sequence $(3,3, \underbrace{n-3, \ldots, n-3}_{n-5 \text { times }}, n-2, n-2, n-1)$, i.e., $H_{1}=K_{1,2} \vee\left(K_{n-5}+2 K_{1}\right)$;

2. $H_{2}$ has degree sequence $(3,3, n-4, \underbrace{n-3, \ldots, n-3}_{n-6 \text { times }}, n-2, n-1, n-1)$;

3. $H_{3}$ has degree sequence $(3,3, n-4, n-4, \underbrace{n-3, \ldots, n-3}_{n-7 \text { times }}, n-1, n-$ $1, n-1)$, i.e., $H_{3}=K_{3} \vee\left(\left(2 K_{1} \vee K_{n-7}\right)+2 K_{1}\right)$.

The graphs which correspond to these degree sequences are depicted in Figure 3.1. Let $V_{1}, V_{2}$ and $V_{3}$ be the sets of vertices of $S_{n}^{3}$ with degree $3, n-1$ and $n-3$, respectively. Then $H_{1}$ is the graph obtained from $S_{n}^{3}$ by deleting an edge $u z$ with $\{u, z\} \in V_{2}, H_{2}$ is the graph obtained from $S_{n}^{3}$ by deleting an edge $u z$ with $u \in V_{2}$ and $z \in V_{3}$, and $H_{3}$ is the graph obtained from $S_{n}^{3}$ by deleting an edge $v z$ with $\{v, z\} \in V_{3}$. 


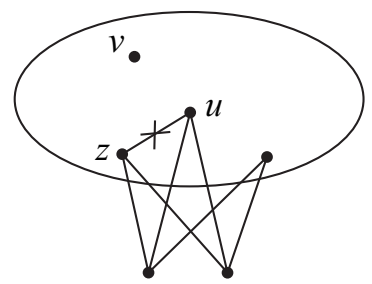

$H_{1}$

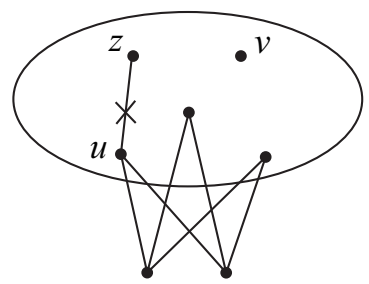

$\mathrm{H}_{2}$

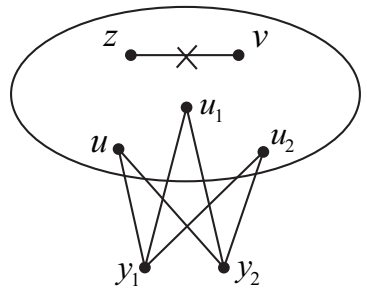

$\mathrm{H}_{3}$

FIGURE 3.1: Graphs obtained from $S_{n}^{3}$ by removing one edge.

Next, we eliminate these graphs by showing that $\rho\left(H_{i}\right)<n-3, i=$ $1,2,3$. Firstly, we claim that $\rho\left(H_{1}\right) \leq \rho\left(H_{2}\right) \leq \rho\left(H_{3}\right)$. Indeed, for graph $H_{i}$, let $u, z \in V\left(H_{i}\right)$ be the two vertices defined above and let $v \in V_{3} \backslash\{u, z\}$, where $i=1,2$. We have $H_{2}=H_{1}-v z+u z$ and $H_{3}=H_{2}-v z+u z$. Thus, using Lemma 1.14, we obtain our claim. Hence it is sufficient to show that $\rho\left(H_{3}\right)<n-3$.

Let us consider the following partition $\pi$ of $V\left(H_{3}\right)$ in $X_{1}=V\left(K_{n-7}\right), X_{2}=$ $\{z, v\}, X_{3}=\left\{u, u_{1}, u_{2}\right\}, X_{4}=\left\{y_{1}, y_{2}\right\}$. It can easily be checked that this partition is equitable with the adjacency matrix of its quotient $H_{3} / \pi$ :

$$
A\left(H_{3} / \pi\right)=\left(\begin{array}{cccc}
n-8 & 2 & 3 & 0 \\
n-7 & 0 & 3 & 0 \\
n-7 & 2 & 2 & 2 \\
0 & 0 & 3 & 0
\end{array}\right) .
$$

The characteristic polynomial $\operatorname{det}\left(x I_{4}-A\left(H_{3} / \pi\right)\right)$ of $A\left(H_{3} / \pi\right)$ is equal to:

$$
f_{1}(x)=x^{4}-(n-6) x^{3}-(3 n-7) x^{2}+4(n-10) x+12 n-84 .
$$

Therefore, by Lemma 1.16, the spectral radius of $H_{3}$ is the largest root of polynomial (3.8).

Next, we will show that there is no root of the polynomial $f_{1}(x)$ in the interval $[n-3,+\infty)$. In fact, when $n \geq 14$, it is obvious that the following 
inequalities are true:

$$
\begin{aligned}
f_{1}(n-3) & =2 n^{2}-28 n+18>0 ; \\
f_{1}^{\prime}(n-3) & =4 x^{3}-3(n-6) x^{2}-2(3 n-7) x+\left.4(n-10)\right|_{x=n-3} \\
& =n(n-3)^{2}-28>0 ; \\
f_{1}^{\prime \prime}(n-3) & =12 x^{2}-6(n-6) x-\left.2(3 n-7)\right|_{x=n-3}=6 n^{2}-24 n+14>0 ; \\
f_{1}^{\prime \prime \prime}(n-3) & =24 x-\left.6(n-6)\right|_{x=n-3}=18(n-2)>0 ; \\
f_{1}^{(4)}(n-3) & =24>0 .
\end{aligned}
$$

Therefore, by the Fourier-Budan theorem [57], all roots of $f_{1}(x)$ lie to the left of the number $n-3$. In particular, $\rho\left(H_{3}\right)<n-3$. Hence, non-Hamiltonconnected graphs $G \in \mathscr{S}_{n}^{3}(1)$ satisfy $\rho(G)<n-3$, a contradiction.

For $G \in \mathscr{S}_{n}^{3}(2)$, by Lemma 1.17, we also have $\rho(G)<n-3$, a contradiction.

For $G \in \mathscr{T}_{n}^{3}(1)$, that is, if $G$ is obtained from the graph $T_{n}^{3}$ by removing one edge, we have to check the following possible degree sequences for graphs $G=T_{i}$ :

1. $T_{1}$ has degree sequence $(3,3, \underbrace{n-3, \ldots, n-3}_{n-4 \text { times }}, n-2, n-2)$, i.e., $T_{1}=$ $2 K_{1} \vee\left(K_{n-4}+K_{2}\right)$;

2. $T_{2}$ has degree sequence $(3,3, n-4, \underbrace{n-3, \ldots, n-3}_{n-5 \text { times }}, n-2, n-1)$;

3. $T_{3}$ has degree sequence $(3,3, n-4, n-4, \underbrace{n-3, \ldots, n-3}_{n-6 \text { times }}, n-1, n-1)$, i.e., $T_{3}=K_{2} \vee\left(\left(2 K_{1} \vee K_{n-6}\right)+K_{2}\right)$.

The graphs which correspond to these degree sequences are depicted in Figure 3.2. Let $V_{1}, V_{2}$ and $V_{3}$ be the sets of vertices of $T_{n}^{3}$ with degree $3, n-1$ and $n-3$, respectively. Then $T_{1}$ is the graph obtained from $T_{n}^{3}$ by deleting an edge $u z$ with $\{u, z\} \in V_{2}, T_{2}$ is the graph obtained from $T_{n}^{3}$ by deleting an edge $u z$ with $u \in V_{2}$ and $z \in V_{3}$, and $T_{3}$ is the graph obtained from $T_{n}^{3}$ by deleting an edge $v z$ with $\{v, z\} \in V_{3}$. 

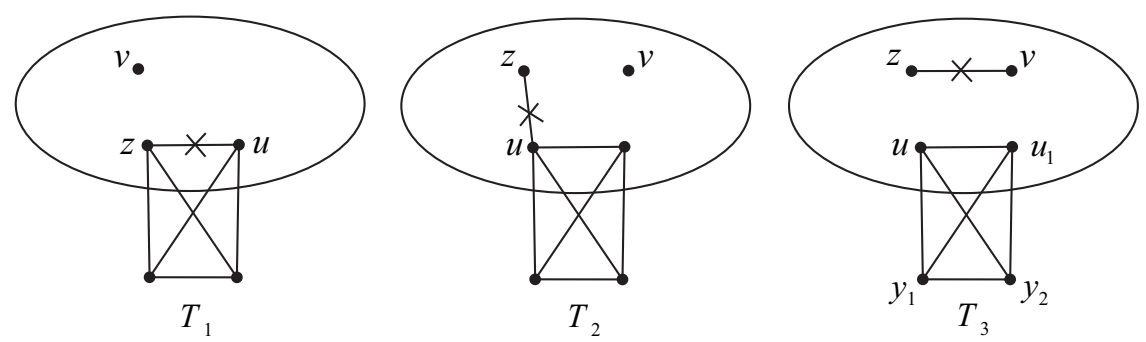

FIGURE 3.2: Graphs obtained from $T_{n}^{3}$ by removing one edge.

Next, we show $\rho\left(T_{i}\right)<n-3, i=1,2,3$. Firstly, we claim that $\rho\left(T_{1}\right) \leq$ $\rho\left(T_{2}\right) \leq \rho\left(T_{3}\right)$. Indeed, for graph $T_{i}$, let $u, z \in V\left(T_{i}\right)$ be the two vertices defined above, and let $v \in V_{3} \backslash\{u, z\}$, where $i=1,2$. We have $T_{2}=T_{1}-v z+u z$ and $T_{3}=T_{2}-v z+u z$. Thus by Lemma 1.14, our claim holds. Hence it is sufficient to show that $\rho\left(T_{3}\right)<n-3$.

Let us consider the following partition $\pi$ of $V\left(T_{3}\right)$ in $X_{1}=V\left(K_{n-6}\right), X_{2}=$ $\{z, v\}, X_{3}=\left\{u, u_{1}\right\}, X_{4}=\left\{y_{1}, y_{2}\right\}$. It can easily be checked that this partition is equitable with the adjacency matrix of its quotient $T_{3} / \pi$ :

$$
A\left(T_{3} / \pi\right)=\left(\begin{array}{cccc}
n-7 & 2 & 2 & 0 \\
n-6 & 0 & 2 & 0 \\
n-6 & 2 & 1 & 2 \\
0 & 0 & 2 & 1
\end{array}\right) .
$$

The characteristic polynomial $\operatorname{det}\left(x I_{4}-A\left(T_{3} / \pi\right)\right)$ of $A\left(T_{3} / \pi\right)$ is equal to:

$$
f_{2}(x)=x^{4}-(n-5) x^{3}-(2 n-3) x^{2}+(5 n-33) x+10 n-56
$$

Therefore, by Lemma 1.16, the spectral radius of $T_{3}$ is the largest root of polynomial (3.9).

Next, we will show that there is no root of the polynomial $f_{2}(x)$ in the interval $[n-3,+\infty)$. In fact, when $n \geq 14$, it is obvious that the following 
inequalities are true:

$$
\begin{aligned}
f_{2}(n-3) & =2 n^{2}-20 n+16>0 \\
f_{2}^{\prime}(n-3) & =4 x^{3}-3(n-5) x^{2}-2(2 n-3) x+5 n-\left.33\right|_{x=n-3} \\
& =(n-3)^{2}(n-2)+n^{2}-7 n-6>0 \\
f_{2}^{\prime \prime}(n-3) & =12 x^{2}-6(n-5) x-\left.2(2 n-3)\right|_{x=n-3} \\
& =2(n-4)(3 n-3)+2 n>0 \\
f_{2}^{\prime \prime \prime}(n-3) & =24 x-\left.6(n-5)\right|_{x=n-3}=6(3 n-7)>0 \\
f_{2}^{(4)}(n-3) & =24>0 .
\end{aligned}
$$

Therefore, by the Fourier-Budan theorem [57], all roots of $f_{2}(x)$ lie to the left of the number $n-3$. In particular, $\rho\left(T_{3}\right)<n-3$. Hence, non-Hamiltonconnected graphs $G \in \mathscr{T}_{n}^{3}(1)$ satisfy $\rho(G)<n-3$, a contradiction.

For $n=14, G=S_{14}^{7}$, and by direct calculation, we have $\rho\left(S_{14}^{7}\right)=$ $10.6158<14-3=11$, a contradiction.

This completes the proof of Theorem 3.6.

The proof of Theorem 3.8. Combining the bounds in Lemma 1.19 and Theorem 3.8, we have $2 n-6+\frac{6}{n-1}<q(G) \leq \frac{2 m}{n-1}+n-2$, so $2 m>n^{2}-5 n+10$, which, by using parity, is equivalent to $2 m \geq n^{2}-5 n+12=\left(\begin{array}{c}n-2 \\ 2\end{array}\right)+3$. Now, supposing that $G$ is not Hamilton-connected, by Corollary 3.3, $G \in$ $\left(\bigcup_{i=0}^{3} \mathscr{S}_{n}^{3}(i)\right) \bigcup\left(\bigcup_{i=0}^{2} \mathscr{T}_{n}^{3}(i)\right)$, or for $n=13, G=S_{13}^{6}$, or for $n=14$, $G \in \bigcup_{i=0}^{1} S_{14}^{7}(i)$.

For $G=S_{n}^{3}$, it has been shown that $q\left(S_{n}^{3}\right)$ is the largest zero of the function $g_{1}(x)=x^{3}-(3 n-5) x^{2}+\left(2 n^{2}-n-24\right) x-6(n-3)(n-4)$ in [70]. Noting that $g_{1}\left(2 n-6+\frac{6}{n-1}\right)=-\frac{18\left(3 n^{3}-18 n^{2}+47 n-44\right)}{(n-1)^{3}}<0$ holds for $n \geq 13$, we obtain $q\left(S_{n}^{3}\right)>2 n-6+\frac{6}{n-1}$, so $S_{n}^{3}$ enters the list of exceptions of the theorem. As in the former proof, we continue by eliminating the other possible cases. We adopt the notation of the former proof.

For $G \in \mathscr{S}_{n}^{3}(1)$, by the discussion in the proof of Theorem 3.6 and using Lemma 1.15, we have $q\left(H_{1}\right) \leq q\left(H_{2}\right) \leq q\left(H_{3}\right)$. So it is sufficient to show that 
$q\left(H_{3}\right)<2 n-6+\frac{6}{n-1}$. Recall that the $(q, X)$-eigenequation in $G$ is

$$
\left[q-d_{G}(v)\right] X_{v}=\sum_{u \in N_{G}(v)} X_{u}
$$

for each $v \in V(G)$, where $X$ is an eigenvector of $Q(G)$ corresponding to the eigenvalue $q$, and $X_{u}$ is the entry of $X$ corresponding to the vertex $u$. For $G=H_{3}$, let $X=\left(x_{1}, x_{2}, \ldots, x_{n}\right)^{T}$ be the eigenvector corresponding to $q(G)$. Then all vertices of degree $n-1$ have the same values given by $X$, say $X_{1}$; all vertices of degree $n-3$ have the same values given by $X$, say $X_{2}$; all vertices of degree $n-4$ have the same values given by $X$, say $X_{3}$. Denote by $X_{4}$ the values of the vertices of degree 3 given by $X$. Assume $\tilde{X}=\left(X_{1}, X_{2}, X_{3}, X_{4}\right)^{T}$, by (3.10), we have

$$
\begin{aligned}
(q(G)-(n-1)) X_{1} & =2 X_{1}+(n-7) X_{2}+2 X_{3}+2 X_{4} ; \\
(q(G)-(n-3)) X_{2} & =3 X_{1}+(n-8) X_{2}+2 X_{3} ; \\
(q(G)-(n-4)) X_{3} & =3 X_{1}+(n-7) X_{2} ; \\
(q(G)-3) X_{4} & =3 X_{1} .
\end{aligned}
$$

Transforming the above equations into a matrix equation $(A-q(G) I) \tilde{X}=$ 0 , we get

$$
A=\left(\begin{array}{cccc}
n+1 & n-7 & 2 & 2 \\
3 & 2 n-11 & 2 & 0 \\
3 & n-7 & n-4 & 0 \\
3 & 0 & 0 & 3
\end{array}\right) .
$$

Thus, $q(G)$ is the largest root of the following equation:

$$
\begin{aligned}
q^{4}-(4 n-11) q^{3} & +\left(5 n^{2}-24 n+10\right) q^{2}-\left(2 n^{3}-7 n^{2}-56 n+220\right) q \\
& +6\left(n^{3}-13 n^{2}+56 n-80\right)=0
\end{aligned}
$$

Let $g_{2}(x)=x^{4}-(4 n-11) x^{3}+\left(5 n^{2}-24 n+10\right) x^{2}-\left(2 n^{3}-7 n^{2}-56 n+220\right) x+$ $6\left(n^{3}-13 n^{2}+56 n-80\right)$. Note that $g_{2}\left(2 n-6+\frac{6}{n-1}\right)=\frac{2\left(4 n^{6}-77 n^{5}+445 n^{4}-1471 n^{3}\right.}{(n-1)^{4}}+$ $\frac{2939 n^{2}-3856 n+2664}{(n-1)^{4}}>0$ for $n \geq 13$. This implies $q\left(H_{3}\right)<2 n-6+\frac{6}{n-1}$. Hence $q\left(H_{1}\right) \leq q\left(H_{2}\right) \leq q\left(H_{3}\right)<2 n-6+\frac{6}{n-1}$, a contradiction. 
For $G \in \mathscr{S}_{n}^{3}(2)$, which is obtained from $S_{n}^{3}$ by deleting two edges, by Lemma 1.17, we also have $q(G)<2 n-6+\frac{6}{n-1}$, a contradiction.

For $G \in \mathscr{S}_{n}^{3}(3)$, which is obtained from $S_{n}^{3}$ by deleting three edges, by Lemma 1.17, we also have $q(G)<2 n-6+\frac{6}{n-1}$, a contradiction.

For $G=T_{n}^{3}$, by a similar method as above, we get that $q(G)$ is the largest zero of the function $g_{3}(x)=x^{3}-(3 n-4) x^{2}+2\left(n^{2}+n-14\right) x-8\left(n^{2}-6 n+8\right)$. Note that $g_{3}\left(2 n-6+\frac{6}{n-1}\right)=\frac{4\left(n^{4}-19 n^{3}+102 n^{2}-250 n+220\right)}{(n-1)^{3}}>0$ for $n \geq 13$. This implies that $q\left(T_{n}^{3}\right)<2 n-6+\frac{6}{n-1}$.

For $G \in \bigcup_{i=1}^{2} \mathscr{T}_{n}^{3}(i), G$ is obtained from $T_{n}^{3}$ by deleting $i \in\{1,2\}$ edges. By Lemma 1.17, we also have $q(G)<2 n-6+\frac{6}{n-1}$, a contradiction.

For $n=13, G=S_{13}^{6}$, and by direct calculation we have $q\left(S_{13}^{6}\right)=20.1157<$ $2 n-6+\frac{6}{n-1}$, a contradiction.

For $n=14, G=S_{14}^{7}$, and by direct calculation we have $q\left(S_{14}^{7}\right)=22.2195<$ $2 n-6+\frac{6}{n-1}$, a contradiction. Finally, for $G \in \mathscr{S}_{14}^{7}(1), G$ is obtained from $S_{14}^{7}$ by deleting one edge, and by Lemma 1.17, we also have $q(G)<2 n-6+\frac{6}{n-1}$, a contradiction.

This completes the proof of Theorem 3.8. 


\section{Chapter 4}

\section{Hamilton-connectivity of graphs with large minimum degree}

In the former chapter, we considered sufficient spectral conditions for graphs with minimum degree $\delta(G) \geq 3$ to be Hamilton-connected. In this chapter, we mainly focus on such conditions for graphs with minimum degree $\delta(G) \geq$ $k$, for a general fixed integer $k \geq 2$. We present a new sufficient spectral condition, in terms of the signless Laplacian spectral radius, for such graphs to be Hamilton-connected.

\subsection{Introduction}

As we have seen in the former chapters, the work of Fiedler and Nikiforov [25] motivated a lot of subsequent work by different research groups on spectral conditions that guarantee certain hamiltonian properties. We refer to the most recent developments in $[2,46,47,53,64-66,69,70]$. We mention a few of these developments in the most recent papers more explicitly, since they are directly related to the new result we will present here. 
Recently, by involving the minimum degree of the graph as a new parameter, Li and Ning $[41,42]$ extended some of the hamiltonicity results in $[25,46,53]$. Their results were in turn further improved by Nikiforov [52], Chen et al. [10], Ge et al. [26], and Li et al. [40]. We omit the details since we focus on Hamilton-connectivity instead of hamiltonicity in this chapter.

Before introducing our main result, let us recall the definitions of two relevant families of graphs that we already encountered in Chapter 3 . For $n \geq 5$ and $1 \leq k \leq n / 2$, we defined:

$$
S_{n}^{k}=K_{k} \vee\left(K_{n-(2 k-1)}+(k-1) K_{1}\right) \text { and } T_{n}^{k}=K_{2} \vee\left(K_{n-(k+1)}+K_{k-1}\right) .
$$

The graph $S_{n}^{k}$ can also be obtained from $K_{n-k+1}$ and $(k-1) K_{1}$ by joining all vertices of $(k-1) K_{1}$ to all vertices of a $k$-subset of $K_{n-k+1}$. The graph $T_{n}^{k}$ can be obtained from $K_{n-k+1}$ and $K_{k+1}$ by identifying two vertices.

For the graph $S_{n}^{k}$, let $X=\left\{v \in V\left(S_{n}^{k}\right) \mid d(v)=k\right\}, Y=\left\{v \in V\left(S_{n}^{k}\right) \mid d(v)=\right.$ $n-1\}$, and $Z=\left\{v \in V\left(S_{n}^{k}\right) \mid d(v)=n-k\right\}$. Let $E_{0}$ be the subset of $E\left(S_{n}^{k}\right)$ containing all edges both end vertices of which are from $Y \cup Z$. For the graph $T_{n}^{k}$, let $X=\left\{v \in V\left(T_{n}^{k}\right) \mid d(v)=k\right\}, Y=\left\{v \in V\left(T_{n}^{k}\right) \mid d(v)=n-1\right\}$, and $Z=\left\{v \in V\left(T_{n}^{k}\right) \mid d(v)=n-k\right\}$. Let $E_{0}$ be the subset of $E\left(T_{n}^{k}\right)$ containing all edges both end vertices of which are from $Y \cup Z$. For a real number $x$, let $\lfloor x\rfloor$ denote the largest integer that is less than or equal to $x$ (usually called the floor of $x$ ). Then we define two new classes of (edge deleted) spanning subgraphs of the above graphs, as follows.

$$
\begin{gathered}
\mathscr{S}_{k}^{(1)}(n)=\left\{G \subseteq S_{n}^{k} \mid G=S_{n}^{k}-E^{\prime}, \text { where } E^{\prime} \subseteq E_{0} \text { with }\left|E^{\prime}\right| \leq\left\lfloor\frac{k(k-1)}{4}\right\rfloor\right\} . \\
\mathscr{T}_{k}^{(1)}(n)=\left\{G \subseteq T_{n}^{k} \mid G=T_{n}^{k}-E^{\prime}, \text { where } E^{\prime} \subseteq E_{0} \text { with }\left|E^{\prime}\right| \leq\left\lfloor\frac{k-1}{2}\right\rfloor\right\} .
\end{gathered}
$$

Our main contribution of this chapter is the following result for graphs with minimum degree $\delta \geq k$, for some integer $k \geq 2$.

Theorem 4.1. Let $G$ be a graph of order $n \geq k^{4}+5 k^{3}+2 k^{2}+8 k+12$ with $\delta(G) \geq k$, where $k \geq 2$. If $q(G) \geq 2 n-2 k$, then $G$ is Hamilton-connected unless $G \in \mathscr{S}_{k}^{(1)}(n) \cup \mathscr{T}_{k}^{(1)}(n)$. 
To prove Theorem 4.1, we also need the following two families of spanning subgraphs of $S_{n}^{k}$ and $T_{n}^{k}$, respectively.

$$
\begin{gathered}
\mathscr{S}_{k}^{(2)}(n)=\left\{G \subseteq S_{n}^{k} \mid G=S_{n}^{k}-E^{\prime} \text {, where } E^{\prime} \subseteq E_{0} \text { with }\left|E^{\prime}\right|=\left\lfloor\frac{k(k-1)}{4}\right\rfloor+1\right\} . \\
\mathscr{T}_{k}^{(2)}(n)=\left\{G \subseteq T_{n}^{k} \mid G=T_{n}^{k}-E^{\prime} \text {, where } E^{\prime} \subseteq E_{0} \text { with }\left|E^{\prime}\right|=\left\lfloor\frac{k-1}{2}\right\rfloor+1\right\} .
\end{gathered}
$$

The rest of the chapter is organized as follows. In Section 4.2, we present two essential known equalities from linear algebra together with two useful lemmas. In Section 4.3, we present the main theorems, lemmas, and their proofs. In Section 4.4, we give a proof for the crucial inequality (4.4) that we use in Section 4.3.

\subsection{Preliminaries}

By Rayleigh's principle (See, e.g., [32]), we have

$$
q(G)=\max _{\mathbf{x}} \frac{\langle Q(G) \mathbf{x}, \mathbf{x}\rangle}{\langle\mathbf{x}, \mathbf{x}\rangle},
$$

where $q(G)$ is the largest eigenvalue of $Q(G)$. By the famous Perron-Frobenius theorem (See, e.g., [27]), any eigenvector $\mathbf{f}$ corresponding to $q(G)$ has $\mathbf{f}_{v}>0$ for each $v \in V(G)$. It is easy to get

$$
(q(G)-d(v)) \mathbf{f}_{v}=\sum_{u \sim v} \mathbf{f}_{u}
$$

where $u \sim v$ indicates that $u$ and $v$ are neighbors.

Next we recall the definition of the closure of $G$, which was introduced by Bondy and Chvátal [5]. For an integer $k$, the $k$-closure of a graph $G$ is the graph obtained from $G$ by successively joining pairs of nonadjacent vertices whose degree sum is at least $k$ until no such pair remains. We denote this $k$-closure of $G$ by $c l_{k}(G)$. Note that $d_{c l_{k}(G)}(u)+d_{c l_{k}(G)}(v) \leq k-1$ for any pair of nonadjacent vertices $u, v \in c l_{k}(G)$, and that $G$ is a spanning subgraph of $c l_{k}(G)$. For Hamilton-connected graphs, the following two counterparts of 
the hamiltonicity results that we mentioned earlier in the thesis are relevant for the sequel.

Lemma 4.2. (Ore [55]) If $G$ is a 2-connected graph of order $n$ and $d(u)+$ $d(v) \geq n+1$ for any two distinct nonadjacent vertices $u$ and $v$, then $G$ is Hamilton-connected.

Lemma 4.3. (Bondy and Chvátal [5]) A graph $G$ of order $n$ is Hamiltonconnected if and only if $\mathrm{cl}_{n+1}(G)$ is Hamilton-connected.

In the next section, we present several results that are necessary for our proof of Theorem 4.1. The following notation will be used in the proofs. We denote by $E\left(V_{1}, V_{2}\right)$ the set of all edges with one end vertex in $V_{1}$ and the other end vertex in $V_{2}$, and we let $e\left(V_{1}, V_{2}\right)=\left|E\left(V_{1}, V_{2}\right)\right|$.

\subsection{Main results and proofs}

Our first result is the basis for our proof of Theorem 4.1. The following notation will be used in its proof. Let $P_{u v}$ and $P_{w z}$ be two vertex-disjoint paths. We denote by $P_{u v} \bigsqcup P_{w z}$ the path obtained from $P_{u v}$ and $P_{w z}$ by joining $v$ and $w$ with an edge.

Theorem 4.4. Let $G$ be a graph of order $n \geq 11 k$ with $\delta(G) \geq k \geq 2$. If $e(G)>\left(\begin{array}{c}n-k \\ 2\end{array}\right)+k(k+1)$, then $G$ is Hamilton-connected unless $G \subseteq S_{n}^{k}$ or $T_{n}^{k}$

Proof. Let $G^{\prime}=c l_{n+1}(G)$. Obviously, we have $\delta\left(G^{\prime}\right) \geq \delta(G)$ and $e\left(G^{\prime}\right) \geq$ $e(G)$. If $G^{\prime}$ is Hamilton-connected, then so is $G$, by Lemma 4.3. Now, we suppose that $G^{\prime}$ is not Hamilton-connected. We first prove the following claim. Recall that $\omega(G)$ denotes the cardinality of a largest clique of $G$.

Claim 1. $\omega\left(G^{\prime}\right)=n-k+1$.

The proof of Claim 1. Let $K$ be the subset of $V\left(G^{\prime}\right)$ containing all the vertices which have degree at least $(n+1) / 2$. By the definition of $c l_{n+1}(G)$, any two 
distinct vertices of $K$ are adjacent in $G^{\prime}$. Let $C$ be (the vertex set of) a largest clique of $G^{\prime}$ containing all the vertices of $K$, and let $|C|=t$ and $H=G^{\prime}-C$.

Firstly, suppose $1 \leq t \leq \frac{2}{3} n+\frac{1}{2}$. For every $v \in V(H)$, we have $d_{C}(v) \leq t-1$ and $d_{G^{\prime}}(v) \leq \frac{n}{2}$. Note that

$$
e(H)+e(V(H), V(C))=\frac{\sum_{v \in V(H)} d_{G^{\prime}}(v)+\sum_{v \in V(H)} d_{C}(v)}{2} .
$$

Hence,

$$
\begin{aligned}
e\left(G^{\prime}\right) & =e\left(G^{\prime}[C]\right)+e(H)+e(V(H), V(C)) \\
& \leq\left(\begin{array}{l}
t \\
2
\end{array}\right)+\frac{\left(\frac{n}{2}+t-1\right)(n-t)}{2} \\
& =\frac{n}{4} t+\frac{n(n-2)}{4} \\
& \leq \frac{n}{4}\left(\frac{2}{3} n+\frac{1}{2}\right)+\frac{n(n-2)}{4} \\
& =\frac{5}{12} n^{2}-\frac{3}{8} n \\
& <\left(\begin{array}{c}
n-k \\
2
\end{array}\right)+k(k+1) \\
& <e\left(G^{\prime}\right),
\end{aligned}
$$

which is a contradiction.

Next, suppose that $\frac{2}{3} n+\frac{1}{2}<t \leq n-k$. For every $v \in V(H)$, we have $d_{G^{\prime}}(v) \leq n-t+1$, since otherwise $v$ would be adjacent to every vertex in $C$. Note that

$$
e(H)+e(V(H), V(C)) \leq \sum_{v \in V(H)} d_{G^{\prime}}(v)
$$

Thus,

$$
\begin{aligned}
e\left(G^{\prime}\right) & =e\left(G^{\prime}[C]\right)+e(H)+e(V(H), V(C)) \\
& \leq\left(\begin{array}{l}
t \\
2
\end{array}\right)+(n-t+1)(n-t)
\end{aligned}
$$




$$
\begin{aligned}
& =\frac{3}{2} t^{2}-\left(2 n+\frac{3}{2}\right) t+n^{2}+n \\
& \leq\left(\begin{array}{c}
n-k \\
2
\end{array}\right)+k(k+1) \\
& <e\left(G^{\prime}\right),
\end{aligned}
$$

which is a contradiction.

So, we conclude that $t \geq n-k+1$ and hence $\omega\left(G^{\prime}\right) \geq n-k+1$.

To complete the proof of Claim 1, suppose that $\omega\left(G^{\prime}\right) \geq n-k+2$. Let $C^{\prime}$ be a largest clique in $G^{\prime}$, and let $H^{\prime}=G^{\prime}-C^{\prime}$. Since $G^{\prime}$ is not Hamiltonconnected, $G^{\prime}$ is not a clique, hence $V\left(H^{\prime}\right) \neq \emptyset$. Let $v$ be a vertex in $V\left(H^{\prime}\right)$. Then $v$ is adjacent to every vertex of $C^{\prime}$ in $G^{\prime}$ since $d_{G^{\prime}}(u) \geq n-k+1$ for every $u \in C^{\prime}$ and $d_{G^{\prime}}(v) \geq \delta\left(G^{\prime}\right) \geq k$, which contradicts that $C^{\prime}$ is a largest clique in $G^{\prime}$. So $\omega\left(G^{\prime}\right)=n-k+1$, as we claimed.

Every time we write degree (or neighbor) in the remainder of this proof, we mean the degree (or a neighbor) in the graph $G^{\prime}$, without specifying this explicitly. We adopt the notation we used in the proof of the above claim, so we let $C^{\prime}$ be a largest clique in $G^{\prime}$, and we let $H^{\prime}=G^{\prime}-C^{\prime}$. Note that every vertex of $C^{\prime}$ has degree at least $n-k$. Let $F=\left\{u_{1}, u_{2}, \ldots, u_{s}\right\}$ be the subset of $C^{\prime}$ containing all the vertices that have degree at least $n-k+1$. In other words, every vertex of $F$ has at least one neighbor in $H^{\prime}$. Then we claim that every vertex of $H^{\prime}$ has degree exactly $k$. We conclude this from $G^{\prime}=c l_{n+1}(G)$ and $\delta\left(G^{\prime}\right) \geq k$. Hence, every vertex of $H^{\prime}$ is adjacent to every vertex in $F$. Moreover, since $\left|V\left(H^{\prime}\right)\right|=k-1$, it is easy to check that $2 \leq s \leq k$. We consider the cases that $s=2, s=k$, and $3 \leq s \leq k-1$ separately.

If $s=2$, then $H^{\prime}$ is a clique and $G^{\prime}=T_{n}^{k}$. If $s=k$, then $H^{\prime}$ is an independent set and $G=S_{n}^{k}$.

Next, we suppose $3 \leq s \leq k-1$, and we complete the proof by showing that $G^{\prime}$ is Hamilton-connected.

By the assumptions, it is clear that $H^{\prime}$ is $(k-s)$-regular, $\left|V\left(H^{\prime}\right)\right|=k-1$, and $G^{\prime}=K_{s} \vee\left(K_{n-k-s+1}+H^{\prime}\right)$, in which $F=V\left(K_{s}\right)$ and $C^{\prime}=K_{s} \vee\left(K_{n-k-s+1}\right)$. Let $W=K_{s} \vee H^{\prime}$. Then $d_{W}\left(w_{1}\right)+d_{W}\left(w_{2}\right) \geq 2 k \geq s+1+k>|W|+1$ for any two nonadjacent vertices $w_{1}$ and $w_{2}$ in $W$. Hence by Lemma $4.2, W$ 
is Hamilton-connected. Then it is obvious that for any two distinct vertices $u \in V\left(K_{n-k-s+1}\right)$ and $v \in V\left(G^{\prime}\right)$, there is a Hamilton path connecting $u$ and $v$ in $G^{\prime}$.

For any two distinct vertices $u, v \in F$, we show there is a Hamilton path connecting them in $G^{\prime}$. We first assume that $H^{\prime}$ is connected. Then $H^{\prime}$ has a cycle $C=u_{1} u_{2} \cdots u_{t} u_{1}$ of order $t \geq k-s+1$, since every vertex in $H^{\prime}$ has degree $k-s$ in $H^{\prime}$. Let $V\left(H^{\prime}\right) \backslash V(C)=\left\{v_{1}, v_{2}, \ldots, v_{k-t-1}\right\}$. Define $F \backslash\{u, v\}=$ $\left\{a_{1}, a_{2}, \ldots, a_{s-2}\right\}$ and $V\left(K_{n-k-s+1}\right)=\left\{b_{1}, b_{2}, \ldots, b_{n-k-s+1}\right\}$. If $C$ contains every vertex of $H^{\prime}$, then $u u_{1} u_{2} \ldots u_{t} a_{1} a_{2} \ldots a_{s-2} b_{1} \cdots b_{n-k-s+1} v$ is a Hamilton path connecting $u$ and $v$. If not, using that $H^{\prime}$ is connected, we suppose $u_{t} v_{1} \in E\left(G^{\prime}\right)$. Then $u u_{1} u_{2} \ldots u_{t} v_{1} a_{1} v_{2} a_{2} \ldots v_{k-t-1} a_{k-t-1} \ldots a_{s-2} b_{1} \cdots b_{n-k-s+1}$ $v$ is a Hamilton path connecting $u$ and $v$ in $G^{\prime}$.

Next, we assume that $H^{\prime}$ is not connected, hence it has at least two components. Every component is $(k-s)$-regular. Hence, there exist two cycles $Q_{1}=u_{11} u_{12} \cdots u_{1 t} u_{11}$ and $Q_{2}=u_{21} u_{22} \cdots u_{2 \ell} u_{21}$ in $H^{\prime}$, where $t \geq k-s+1$ and $\ell \geq k-s+1$. Let $V\left(H^{\prime}\right) \backslash\left(V\left(Q_{1}\right) \cup V\left(Q_{2}\right)\right)=\left\{v_{1}, v_{2}, \ldots, v_{k-t-\ell-1}\right\}$. Note that $t+\ell \geq k-s+3$. Let $R_{1}=u u_{11} \ldots u_{1 t} a_{s-3} u_{21} \ldots u_{2 \ell} a_{s-2}$ and $R_{2}=v_{1} a_{1} v_{2} a_{2} \ldots v_{k-t-\ell-1} a_{k-t-\ell-1} \ldots a_{s-4} b_{1} \cdots b_{n-k-s+1} v$. Then $R_{1} \bigsqcup R_{2}$ is a Hamilton path connecting $u$ and $v$ in $G^{\prime}$.

For any two distinct vertices $u \in F$ and $v \in V\left(H^{\prime}\right)$, or $u, v \in V\left(H^{\prime}\right)$, we can use similar arguments to show that there is a Hamilton path connecting them in $G^{\prime}$. We omit the details.

Hence if $3 \leq s \leq k-1$, then $G^{\prime}$ is Hamilton-connected, which is a contradiction to our assumption. So, we conclude that $G \subseteq S_{n}^{k}$ or $T_{n}^{k}$.

This completes the proof of Theorem 4.4.

Remark 4.1. Recently, we got aware of the fact that a result similar to Theorem 4.4 was obtained independently in [59] (See Theorem 2.5 in [59]).

Next, we present and prove two lemmas about the signless Laplacian spectral radius of the families of graphs we introduced at the beginning of this chapter.

Lemma 4.5. For each $G \in \mathscr{S}_{k}^{(1)}(n) \cup \mathscr{T}_{k}^{(1)}(n)$, we have $q(G) \geq 2 n-2 k$. 
Proof. For $G \in \mathscr{S}_{k}^{(1)}(n)$, we define a column vector $\mathbf{c}$ such that $\mathbf{c}_{u}=1$ for all $u \in Y \cup Z$ and $\mathbf{c}_{v}=0$ for all $v \in X$. Note that $\mathbf{c}$ is an eigenvector corresponding to the eigenvalue $2 n-2 k$ of $(k-1) K_{1}+K_{n-k+1}$. Then we have

$$
\langle Q(G) \mathbf{c}, \mathbf{c}\rangle-\left\langle Q\left((k-1) K_{1}+K_{n-k+1}\right) \mathbf{c}, \mathbf{c}\right\rangle=k(k-1)-4\left|E^{\prime}\right| \geq 0 .
$$

By Rayleigh's principle, we get $q(G) \geq 2 n-2 k$.

Similarly, for $G \in \mathscr{T}_{k}^{(1)}(n)$, we have

$$
\langle Q(G) \mathbf{c}, \mathbf{c}\rangle-\left\langle Q\left((k-1) K_{1}+K_{n-k+1}\right) \mathbf{c}, \mathbf{c}\right\rangle=2(k-1)-4\left|E^{\prime}\right| \geq 0 .
$$

By Rayleigh's principle, we can also get $q(G) \geq 2 n-2 k$.

Lemma 4.6. Let $G$ be a graph of order $n \geq k^{4}+5 k^{3}+2 k^{2}+8 k+12$ with $\delta(G) \geq k \geq 2$. For each $G \in \mathscr{S}_{k}^{(2)}(n) \cup{T_{k}^{(2)}}^{(n)}$, we have $q(G)<2 n-2 k$.

Proof. Let $G \in \mathscr{S}_{k}^{(2)}(n) \cup \mathscr{T}_{k}^{(2)}(n)$, and let $\mathbf{c}$ be the vector defined in the proof in Lemma 4.5. We first prove the following claim.

Claim 1. $q(G)>2 n-2 k-1$.

The proof of Claim 1. If $G \in \mathscr{S}_{k}^{(2)}(n)$, then we have

$$
\langle Q(G) \mathbf{c}, \mathbf{c}\rangle-\left\langle Q\left((k-1) K_{1}+K_{n-k+1}\right) \mathbf{c}, \mathbf{c}\right\rangle=k(k-1)-4\left|E^{\prime}\right| \geq-4,
$$

which implies that $q(G) \geq 2 n-2 k-\frac{4}{\|\mathbf{c}\|^{2}}>2 n-2 k-1$.

If $G \in \mathscr{T}_{k}^{(2)}(n)$, then we have

$$
\langle Q(G) \mathbf{c}, \mathbf{c}\rangle-\left\langle Q\left((k-1) K_{1}+K_{n-k+1}\right) \mathbf{c}, \mathbf{c}\right\rangle=2(k-1)-4\left|E^{\prime}\right| \geq-4,
$$

which implies that $q(G) \geq 2 n-2 k-\frac{4}{\|\mathbf{c}\|^{2}}>2 n-2 k-1$.

In the following part of the proof, we will assume that $G \in \mathscr{S}_{k}^{(2)}(n)$. Since the proofs for the case $G \in \mathscr{T}_{k}^{(2)}(n)$ are very similar to those in the case $G \in \mathscr{S}_{k}^{(2)}(n)$, we only give a rough sketch of the proof for this other case.

Let $G \in \mathscr{S}_{k}^{(2)}(n)$ have the maximum signless Laplacian spectral radius. Let $\mathbf{f}$ be an eigenvector corresponding to $q(G)$ chosen in such a way that 
$\max _{v \in V(G)} \mathbf{f}_{v}=1$. This is possible due to the Perron-Frobenius theorem that we mentioned in the preliminaries.

Recall that $E^{\prime}$ is the set of all edges with both end vertices in $Y \cup Z$. We define two subsets of $Y$ and $Z$, respectively, as follows.

$$
\begin{gathered}
Y_{1}=\{y \in Y \mid d(y)=n-1\} \text { and } Y_{2}=\{y \in Y \mid d(y) \leq n-2\} ; \\
Z_{1}=\{y \in Z \mid d(y)=n-k\} \text { and } Z_{2}=\{y \in Z \mid d(y) \leq n-k-1\} .
\end{gathered}
$$

Since $n \geq k^{4}+5 k^{3}+2 k^{2}+8 k+12, Z_{1} \neq \emptyset$. We next prove a number of claims.

Claim 2. $\mathbf{f}_{x} \leq \frac{k}{q(G)-k}$ for each $x \in X$.

The proof of Claim 2. By (4.1), we have $(q(G)-d(x)) \mathbf{f}_{x}=\sum_{y \in Y} \mathbf{f}_{y}$. The claim now follows from our assumption on $\mathbf{f}$ and the fact that $d(x)=k$.

\section{Claim 3.}

(i) If $Y_{1} \neq \emptyset$ and $Y_{2} \neq \emptyset$, then for any $u \in Y_{1}, v \in Y_{2} \cup Z_{1}$, we have $\mathbf{f}_{u}>\mathbf{f}_{v}$.

(ii) If $Z_{2} \neq \emptyset$ and $Y_{2} \neq \emptyset$, then for any $u \in Z_{1}, v \in Z_{2} \cup Y_{2}$, we have $\mathbf{f}_{u}>\mathbf{f}_{v}$.

The proof of Claim 3. For any $u, v \in V(G)$, using (4.1), we have

$$
\begin{aligned}
(q(G)-d(u))\left(\mathbf{f}_{u}-\mathbf{f}_{v}\right)= & (q(G)-d(u)) \mathbf{f}_{u}-(q(G)-d(v)) \mathbf{f}_{v} \\
& +(d(u)-d(v)) \mathbf{f}_{v} \\
= & (d(u)-d(v)) \mathbf{f}_{v}+\sum_{s \in N(u)} \mathbf{f}_{s}-\sum_{t \in N(v)} \mathbf{f}_{t} \\
= & (d(u)-d(v)) \mathbf{f}_{v}+\sum_{s \in N(u) \backslash N(v)} \mathbf{f}_{s}-\sum_{t \in N(v) \backslash N(u)} \mathbf{f}_{t} .
\end{aligned}
$$


For any $u \in Y_{1}$ and $v \in Y_{2} \cup Z_{1}$, or for any $u \in Z_{1}$ and $v \in Z_{2}, u v \in E(G)$, and (4.2) is equivalent to the following equation.

$$
(q(G)-d(u)+1)\left(\mathbf{f}_{u}-\mathbf{f}_{v}\right)=(d(u)-d(v)) \mathbf{f}_{v}+\sum_{s \in N(u) \backslash N[v]} \mathbf{f}_{s}-\sum_{t \in N(v) \backslash N[u]} \mathbf{f}_{t} .
$$

Since $N(v) \backslash N[u]=\emptyset$, by (4.3), then

$$
(q(G)-d(u)+1)\left(\mathbf{f}_{u}-\mathbf{f}_{v}\right)=(d(u)-d(v)) \mathbf{f}_{v}+\sum_{s \in N(u) \backslash N[v]} \mathbf{f}_{s}>0,
$$

so $\mathbf{f}_{u}>\mathbf{f}_{v}$.

Since $G$ has the maximum signless Laplacian spectral radius in $\mathscr{S}_{k}^{(2)}(n)$, we claim that $G[Y]$ contains the largest number of edges in $\mathscr{S}_{k}^{(2)}(n)$. If not, since $\left|E^{\prime}\right|=\left\lfloor\frac{k(k-1)}{4}\right\rfloor+1$ and $n \geq k^{4}+5 k^{3}+2 k^{2}+8 k+12$, there always exist $v, w \in Y_{2}$ and $u \in Z_{2}$ such that $v w \notin E(G)$ and $u w \in E(G)$. We construct $G^{*}$ by adding the edges $\left\{v w_{i} \mid w_{i} \in N_{G}(u) \backslash N_{G}[v]\right\}$ and deleting the edges $\left\{u w_{i} \mid w_{i} \in N_{G}(u) \backslash N_{G}[v]\right\}$. Hence, $G^{*}[Y]$ has more edges than $G[Y]$. It is easy to see that the above transformation is a Kelmans transformation. By Lemma 1.15 , we get $q\left(G^{*}\right) \geq q(G)$, which contradicts the choice of $G$. This confirms our claim that $G[Y]$ contains the largest number of edges in $\mathscr{S}_{k}^{(2)}(n)$.

For any $u \in Z_{1}$ and $v \in Y_{2}$, we shall prove $\mathbf{f}_{u}>\mathbf{f}_{v}$. Suppose there exist $u \in Z_{1}$ and $v \in Y_{2}$ such that $\mathbf{f}_{u} \leq \mathbf{f}_{v}$. Let $w \in Y_{2}$ be a vertex not adjacent to $v$. We construct a new graph $G_{0}$ by adding the edge $v w$ and deleting the edge $u w$. Note that

$$
\left.\left.\left\langle Q\left(G_{0}\right) \mathbf{f}, \mathbf{f}\right)\right\rangle-\langle Q(G) \mathbf{f}, \mathbf{f})\right\rangle=\mathbf{f}_{v}^{2}+2 \mathbf{f}_{v} \mathbf{f}_{w}-\mathbf{f}_{u}^{2}-2 \mathbf{f}_{u} \mathbf{f}_{w} \geq 0 .
$$

We get $q\left(G_{0}\right) \geq q(G)$ while $G_{0}[Y]$ has more edges than $G[Y]$, a contradiction.

This completes the proof of Claim 3.

Claim 4. $\max _{v \in Y \cup Z} \mathbf{f}_{v}-\min _{u \in Y \cup Z} \mathbf{f}_{u} \leq \frac{k^{2}+6 k+6}{2(q(G)-n+1)}$. 
The proof of Claim 4. We distinguish the two cases that $Y_{1}$ is empty and nonempty, and we start with the former case.

Case 1. $Y_{1}=\emptyset$.

In this case, by Claim $3, \max _{v \in Y \cup Z} \mathbf{f}_{v}$ is attained by some vertex from $Z_{1}$, say $z$. By the definition of $Z_{1}, z$ is adjacent to the other vertices of $Y \cup Z$.

Suppose first that $\min _{v \in Y \cup Z} \mathbf{f}_{v}$ is attained by some vertex from $Z_{2}$, say $w$. Obviously, we have $z w \in E(G), N(w) \backslash N[z]=\emptyset, d(z)-d(w) \leq\left\lfloor\frac{k(k-1)}{4}\right\rfloor+1$ and $|N(z) \backslash N[w]| \leq\left\lfloor\frac{k(k-1)}{4}\right\rfloor+1$. Hence, by (4.3) we have

$$
\begin{aligned}
(q(G)-d(z)+1)\left(\mathbf{f}_{z}-\mathbf{f}_{w}\right) & =(d(z)-d(w)) \mathbf{f}_{w}+\sum_{s \in N(z) \backslash N[w]} \mathbf{f}_{s} \\
& \leq \frac{k(k-1)}{2}+2 .
\end{aligned}
$$

Since $d(z)=n-k$, we obtain

$$
\mathbf{f}_{z}-\mathbf{f}_{w} \leq \frac{k(k-1)+4}{2(q(G)-n+k+1)}=\frac{k^{2}-k+4}{2(q(G)-n+k+1)}<\frac{k^{2}+6 k+6}{2(q(G)-n+1)} .
$$

Next, suppose that $\min _{v \in Y \cup Z} \mathbf{f}_{v}$ is attained by some vertex from $Y_{2}$, say $w$. Obviously, we have $z w \in E(G), N(w) \backslash N[z]=X$ and $|N(z) \backslash N[w]| \leq$ $\left\lfloor\frac{k(k-1)}{4}\right\rfloor+1$. Furthermore, we observe

$$
d(z)-d(w) \leq\left((n-k)-\left(n-1-\left\lfloor\frac{k(k-1)}{4}\right\rfloor-1\right)\right)=\left\lfloor\frac{k(k-1)}{4}\right\rfloor-k+2 .
$$

Hence, by (4.3) we have

$$
\begin{aligned}
(q(G)-d(z)+1)\left(\mathbf{f}_{z}-\mathbf{f}_{w}\right) & =(d(z)-d(w)) \mathbf{f}_{w}+\sum_{s \in N(z) \backslash N[w]} \mathbf{f}_{s}-\sum_{t \in X} \mathbf{f}_{t} \\
& \leq\left\lfloor\frac{k(k-1)}{4}\right\rfloor-k+2+\left\lfloor\frac{k(k-1)}{4}\right\rfloor+1 \\
& \leq \frac{k(k-1)}{2}-k+3 .
\end{aligned}
$$


Since $d(z)=n-k$, we obtain

$$
\mathbf{f}_{z}-\mathbf{f}_{w} \leq \frac{k^{2}-3 k+6}{2(q(G)-n+k+1)}<\frac{k^{2}+6 k+6}{2(q(G)-n+1)} .
$$

Case 2. $Y_{1} \neq \emptyset$.

In this case, by Claim 3, we obtain that $\max _{v \in V(G)} \mathbf{f}_{v}$ is attained by some vertex from $Y_{1}$, say $z$. By the definition of $Y_{1}, z$ is adjacent to the other vertices of $G$.

Suppose first that $\min _{v \in Y \cup Z} \mathbf{f}_{v}$ is attained by some vertex from $Z_{2}$, say $w$. Obviously, we have $z w \in E(G)$ and $N(w) \backslash N[z]=\emptyset$. Furthermore, we observe that

$$
d(z)-d(w) \leq\left((n-1)-\left(n-k-\left\lfloor\frac{k(k-1)}{4}\right\rfloor-1\right)\right)=\left\lfloor\frac{k(k-1)}{4}\right\rfloor+k,
$$

and

$$
|N(z) \backslash N[w]| \leq(k-1)+\left\lfloor\frac{k(k-1)}{4}\right\rfloor+1=\left\lfloor\frac{k(k-1)}{4}\right\rfloor+k .
$$

Hence, by (4.3) we have

$$
\begin{aligned}
(q(G)-d(z)+1)\left(\mathbf{f}_{z}-\mathbf{f}_{w}\right) & =(d(z)-d(w)) \mathbf{f}_{w}+\sum_{s \in N(z) \backslash N[w]} \mathbf{f}_{s} \\
& \leq\left\lfloor\frac{k(k-1)}{4}\right\rfloor+k+\left\lfloor\frac{k(k-1)}{4}\right\rfloor+k \\
& \leq \frac{k(k-1)}{2}+2 k .
\end{aligned}
$$

Since $d(z)=n-1$, we obtain,

$$
\mathbf{f}_{z}-\mathbf{f}_{w} \leq \frac{k^{2}+3 k}{2(q(G)-n+1+1)}<\frac{k^{2}+6 k+6}{2(q(G)-n+1)} .
$$

Next, suppose $\min _{v \in Y \cup Z} \mathbf{f}_{v}$ is attained by some vertex from $Y_{2}$, say $w$. Obviously, we have $z w \in E(G)$ and $N(w) \backslash N[z]=\emptyset$. Furthermore, we 
observe that

$$
d(z)-d(w) \leq\left((n-1)-\left(n-1-\left\lfloor\frac{k(k-1)}{4}\right\rfloor-1\right)\right)=\left\lfloor\frac{k(k-1)}{4}\right\rfloor+1,
$$

and

$$
|N(z) \backslash N[w]| \leq\left\lfloor\frac{k(k-1)}{4}\right\rfloor+1 .
$$

Hence, by (4.3) we have

$$
\begin{aligned}
(q(G)-d(z)+1)\left(\mathbf{f}_{z}-\mathbf{f}_{w}\right) & =(d(z)-d(w)) \mathbf{f}_{w}+\sum_{s \in N(z) \backslash N[w]} \mathbf{f}_{s} \\
& \leq\left\lfloor\frac{k(k-1)}{4}\right\rfloor+1+\left\lfloor\frac{k(k-1)}{4}\right\rfloor+1 \\
& \leq \frac{k(k-1)}{2}+2 .
\end{aligned}
$$

So,

$$
\mathbf{f}_{z}-\mathbf{f}_{w} \leq \frac{k^{2}-k+4}{2(q(G)-n+1+1)}<\frac{k^{2}+6 k+6}{2(q(G)-n+1)} .
$$

This completes the proof of Claim 4 .

Using the above claims, we have

$$
\begin{aligned}
& \langle Q(G) \mathbf{f}, \mathbf{f}\rangle-\left\langle Q\left((k-1) K_{1}+K_{n-k+1}\right) \mathbf{f}, \mathbf{f}\right\rangle \\
& =\sum_{y \in Y} d_{G[X \cup Y]}(y) \mathbf{f}_{y}^{2}+\sum_{x \in X} d_{G[X \cup Y]}(x) \mathbf{f}_{x}^{2}+2 \sum_{\{x, y\} \in E(G[X \cup Y])} \mathbf{f}_{x} \mathbf{f}_{y} \\
& -\sum_{\{u, v\} \in E^{\prime}}\left(\mathbf{f}_{u}^{2}+\mathbf{f}_{v}^{2}+2 \mathbf{f}_{u} \mathbf{f}_{v}\right) \\
& =\sum_{\{x, y\} \in E(G[X \cup Y])}\left(\mathbf{f}_{x}+\mathbf{f}_{y}\right)^{2}-\sum_{\{u, v\} \in E^{\prime}}\left(\mathbf{f}_{u}+\mathbf{f}_{v}\right)^{2} \\
& \leq k(k-1)\left(1+\frac{k}{q(G)-k}\right)^{2}-4\left|E^{\prime}\right|\left(1-\frac{k^{2}+6 k+6}{2(q(G)-n+1)}\right)^{2} \\
& <0 .
\end{aligned}
$$

Here, the last inequality follows by tedious calculations from $n \geq k^{4}+$ 
$5 k^{3}+2 k^{2}+8 k+12$ and $q(G)>2 n-2 k-1$. We have put these final calculations referring to (4.4) in an appendix to this chapter (Section 4.4). Note that $q\left((k-1) K_{1}+K_{n-k+1}\right)=2 n-2 k>\langle Q(G) \mathbf{f}, \mathbf{f}\rangle /\langle\mathbf{f}, \mathbf{f}\rangle$. Hence, $q(G)<2 n-2 k$ for $G \in \mathscr{S}_{k}^{(2)}(n)$. This completes the proof for $G \in \mathscr{S}_{k}^{(2)}(n)$.

For $G \in \mathscr{T}_{k}^{(2)}(n)$, using the same definitions of $Y_{1}, Y_{2}, Z_{1}, Z_{2}$ as for $\mathscr{S}_{k}^{(2)}(n)$, we can obtain the same conclusions as in Claim 2 and Claim 3 by similar methods. Let $G \in \mathscr{T}_{k}^{(2)}(n)$ have the maximum signless Laplacian spectral radius. Let $\mathbf{f}$ be an eigenvector corresponding to $q(G)$. As before, we may assume that $\max _{v \in V(G)} \mathbf{f}_{v}=1$. We can also obtain that

$$
\max _{v \in Y \cup Z} \mathbf{f}_{v}-\min _{u \in Y \cup Z} \mathbf{f}_{u} \leq \frac{k^{2}+6 k+6}{2(q(G)-n+1)} .
$$

To prove this, we use the same case distinction as before.

Case 1. $Y_{1}=\emptyset$.

In this case, $\max _{v \in V(G)} \mathbf{f}_{v}$ is attained by some vertex from $Z_{1}$, say $z$.

Suppose first that $\min _{v \in Y \cup Z} \mathbf{f}_{v}$ is attained by some vertex from $Z_{2}$, say $w$. Obviously, we have $z w \in E(G), N(w) \backslash N[z]=\emptyset, d(z)-d(w) \leq\left\lfloor\frac{k-1}{2}\right\rfloor+1$ and $|N(z) \backslash N[w]| \leq\left\lfloor\frac{k-1}{2}\right\rfloor+1$. Hence, by (4.3) we have

$$
\begin{aligned}
(q(G)-d(z)+1)\left(\mathbf{f}_{z}-\mathbf{f}_{w}\right) & =(d(z)-d(w)) \mathbf{f}_{w}+\sum_{s \in N(z) \backslash N[w]} \mathbf{f}_{s} \\
& \leq k+1 .
\end{aligned}
$$

Next, suppose that $\min _{v \in Y \cup Z} \mathbf{f}_{v}$ is attained by some vertex from $Y_{2}$, say $w$. Obviously, we have $z w \in E(G), N(w) \backslash N[z]=X$ and $|N(z) \backslash N[w]| \leq$ $\left\lfloor\frac{k-1}{2}\right\rfloor+1$. Furthermore, we observe $|d(z)-d(w)| \leq\left\lfloor\frac{k-1}{2}\right\rfloor-k+2$. Hence, by (4.3) we have

$$
\begin{aligned}
(q(G)-d(z)+1)\left(\mathbf{f}_{z}-\mathbf{f}_{w}\right) & =(d(z)-d(w)) \mathbf{f}_{w}+\sum_{s \in N(z) \backslash N[w]} \mathbf{f}_{s}-\sum_{t \in X} \mathbf{f}_{t} \\
& \leq\left\lfloor\frac{k-1}{2}\right\rfloor-k+2+\left\lfloor\frac{k-1}{2}\right\rfloor+1 \\
& \leq 2 .
\end{aligned}
$$


In both subcases, since $d(z)=n-k$, we obtain

$$
\mathbf{f}_{z}-\mathbf{f}_{w} \leq \frac{k^{2}+6 k+6}{2(q(G)-n+1)}
$$

Case 2. $Y_{1} \neq \emptyset$.

In this case, $\max _{v \in V(G)} \mathbf{f}_{v}$ is attained by some vertex from $Y_{1}$. Suppose first that $\min _{v \in Y \cup Z} \mathbf{f}_{v}$ is attained by some vertex from $Z_{2}$, say $w$. Obviously, we have $z w \in E(G), N(w) \backslash N[z]=\emptyset$. Furthermore, we observe that

$$
d(z)-d(w) \leq\left((n-1)-\left(n-k-\left\lfloor\frac{k-1}{2}\right\rfloor-1\right)\right)=\left\lfloor\frac{k-1}{2}\right\rfloor+k,
$$

and

$$
|N(z) \backslash N[w]| \leq(k-1)+\left\lfloor\frac{k-1}{2}\right\rfloor+1=\left\lfloor\frac{k-1}{2}\right\rfloor+k .
$$

Hence, by (4.3) we have

$$
\begin{aligned}
(q(G)-d(z)+1)\left(\mathbf{f}_{z}-\mathbf{f}_{w}\right) & =(d(z)-d(w)) \mathbf{f}_{w}+\sum_{s \in N(z) \backslash N[w]} \mathbf{f}_{s} \\
& \leq\left\lfloor\frac{k-1}{2}\right\rfloor+k+\left\lfloor\frac{k-1}{2}\right\rfloor+k \\
& \leq 3 k-1 .
\end{aligned}
$$

Since $d(z)=n-1$, we obtain,

$$
\mathbf{f}_{z}-\mathbf{f}_{w} \leq \frac{6 k-2}{2(q(G)-n+1+1)}<\frac{k^{2}+6 k+6}{2(q(G)-n+1)} .
$$

Next, suppose that $\min _{v \in Y \cup Z} \mathbf{f}_{v}$ is attained by some vertex from $Y_{2}$, say $w$. Obviously, we have $z w \in E(G)$ and $N(w) \backslash N[z]=\emptyset$. Furthermore, we observe that

$$
d(z)-d(w) \leq\left((n-1)-\left(n-1-\left\lfloor\frac{k-1}{2}\right\rfloor-1\right)\right)=\left\lfloor\frac{k-1}{2}\right\rfloor+1,
$$

and

$$
|N(z) \backslash N[w]| \leq\left\lfloor\frac{k-1}{2}\right\rfloor+1 .
$$


Hence, by (4.3) we have

$$
\begin{aligned}
(q(G)-d(z)+1)\left(\mathbf{f}_{z}-\mathbf{f}_{w}\right) & =(d(z)-d(w)) \mathbf{f}_{w}+\sum_{s \in N(z) \backslash N[w]} \mathbf{f}_{s} \\
& \leq\left\lfloor\frac{k-1}{2}\right\rfloor+1+\left\lfloor\frac{k-1}{2}\right\rfloor+1 \\
& \leq k+1 .
\end{aligned}
$$

So,

$$
\mathbf{f}_{z}-\mathbf{f}_{w} \leq \frac{2 k+2}{2(q(G)-n+1+1)}<\frac{k^{2}+6 k+6}{2(q(G)-n+1)} .
$$

Using the above calculations, for $G \in{\mathscr{T}_{k}^{(2)}}^{(2}(n)$ we can prove $q(G)<2 n-2 k$ by repeating the arguments we used for $G \in \mathscr{S}_{k}^{(2)}(n)$.

This completes the proof of Lemma 4.6.

The proof of Theorem 4.1. Using Theorem 1.19 and the hypothesis of Theorem 4.1, we obtain

$$
2 n-2 k \leq q(G) \leq \frac{2 e(G)}{n-1}+n-2 .
$$

Noting that $n \geq k^{4}+5 k^{3}+2 k^{2}+8 k+12$, we can deduce that

$$
\begin{aligned}
e(G) & \geq \frac{(n-2 k+2)(n-1)}{2} \\
& =\frac{n^{2}-(2 k+1) n+2 n+2 k-2}{2} \\
& =\left(\begin{array}{c}
n-k \\
2
\end{array}\right)+\frac{2 n-k^{2}+k-2}{2} \\
& >\left(\begin{array}{c}
n-k \\
2
\end{array}\right)+k(k+1) .
\end{aligned}
$$

By Theorem 4.4, $G$ is Hamilton-connected unless $G \subseteq S_{n}^{k}$ or $T_{n}^{k}$. Combining this with Lemmas 4.5 and 4.6, we obtain the statement of Theorem 4.1. 
It is easy to see that by performing $k-2$ Kelmans operations on $T_{n}^{k}$, we can obtain a proper subgraph of $S_{n}^{k}$. Hence, by the fact that the signless Laplacian spectral radius decreases after deleting an edge in a connected graph, we obtain $q\left(S_{n}^{k}\right)>q\left(T_{n}^{k}\right)>q\left(K_{n-k+1}\right)=2 n-2 k$. Thus, we immediately obtain the following corollary.

Corollary 4.7. Let $G$ be a graph of order $n \geq k^{4}+5 k^{3}+2 k^{2}+8 k+12$ with $\delta(G) \geq k \geq 2$. If $q(G) \geq q\left(S_{n}^{k}\right)$, then $G$ is Hamilton-connected unless $G=S_{n}^{k}$.

Corollary 4.7 shows that among all graphs of order $n \geq k^{4}+5 k^{3}+2 k^{2}+$ $8 k+12$ with minimum degree $\delta \geq k \geq 2, S_{n}^{k}$ is the extremal non-Hamiltonconnected graph with maximum signless Laplacian spectral radius.

\subsection{Appendix}

The final calculations for the last inequality in (4.4).

Let $A=k(k-1)\left(1+\frac{k}{q(G)-k}\right)^{2}$ and $B=4\left|E^{\prime}\right|\left(1-\frac{k^{2}+6 k+6}{2(q(G)-n+1)}\right)^{2}$. We use $\max A$ and $\min B$ to denote the maximum and minimum values that $A$ and $B$ can attain for different choices of $q(G)$, respectively. Since $q(G) \geq 2 n-2 k-1$, it is clear that

$$
\max A=k(k-1)\left(1+\frac{k}{2 n-3 k-1}\right)^{2} .
$$

If $k=4 s$, then $\left|E^{\prime}\right|=\left\lfloor\frac{4 s(4 s-1)}{4}\right\rfloor+1=s(4 s-1)+1$. If $k=4 s+1$, then $\left|E^{\prime}\right|=\left\lfloor\frac{4 s(4 s+1)}{4}\right\rfloor+1=s(4 s+1)+1$. In both cases, we have

$$
\min B=(k(k-1)+4)\left(1-\frac{k^{2}+6 k+6}{2(n-2 k)}\right)^{2} .
$$

Combining this with (4.5), we use the following deduction and implicitly introduce $A_{1}, A_{2}, A_{3}$, and $A_{4}$.

$$
A-B \leq \max A-\min B
$$




$$
\begin{aligned}
= & k(k-1)\left(1+\frac{k}{2 n-3 k-1}\right)^{2}-k(k-1)\left(1-\frac{k^{2}+6 k+6}{2(n-2 k)}\right)^{2} \\
& -4\left(1-\frac{k^{2}+6 k+6}{2(n-2 k)}\right)^{2} \\
= & A_{1}+A_{2}+A_{3}-A_{4}-4 .
\end{aligned}
$$

In the final above equality,

$$
\begin{aligned}
& A_{1}=\frac{2 k^{3}-2 k^{2}}{2 n-3 k-1} ; \\
& A_{2}=\frac{k^{4}-k^{3}}{4 n^{2}-(12 k+4) n+9 k^{2}+6 k+1} ; \\
& A_{3}=\frac{k^{4}+5 k^{3}+4 k^{2}+18 k+24}{n-2 k} ; \\
& A_{4}=\frac{k^{6}+11 k^{5}+40 k^{4}+72 k^{3}+156 k^{2}+252 k+144}{4 n^{2}-16 k n+16 k^{2}} .
\end{aligned}
$$

Since $n \geq k^{4}+5 k^{3}+2 k^{2}+8 k+12$, we obtain that $A_{1}+A_{2}+A_{3}-A_{4}<4$.

Hence, the final inequality in (4.4) holds.

$$
\text { If } k=4 s+2 \text {, then }\left|E^{\prime}\right|=\left\lfloor\frac{(4 s+2)(4 s+1)}{4}\right\rfloor+1=4 s^{2}+3 s+1 \text {. Hence, }
$$

$$
B>4\left(4 s^{2}+3 s+1\right)\left(1-\frac{k^{2}+3 k+6}{n-2 k}\right)^{2}=(k(k-1)+2)\left(1-\frac{k^{2}+6 k+6}{2(n-2 k)}\right)^{2} .
$$

If $k=4 s+3$, then $\left|E^{\prime}\right|=\left\lfloor\frac{(4 s+3)(4 s+2)}{4}\right\rfloor+1=4 s^{2}+5 s+2$. Hence,

$$
B>4\left(4 s^{2}+5 s+2\right)\left(1-\frac{k^{2}+3 k+6}{n-2 k}\right)^{2}=(k(k-1)+2)\left(1-\frac{k^{2}+6 k+6}{2(n-2 k)}\right)^{2} .
$$

In both cases, combining this with (4.5), we obtain

$$
A-B<\max A-B
$$




$$
\begin{aligned}
< & k(k-1)\left(1+\frac{k}{2 n-3 k-1}\right)^{2}-k(k-1)\left(1-\frac{k^{2}+6 k+6}{2(n-2 k)}\right)^{2} \\
& -2\left(1-\frac{k^{2}+6 k+6}{2(n-2 k)}\right)^{2} \\
= & A_{1}^{\prime}+A_{2}^{\prime}+A_{3}^{\prime}-A_{4}^{\prime}-2 .
\end{aligned}
$$

In the final above equality,

$$
\begin{aligned}
& A_{1}^{\prime}=\frac{2 k^{3}-2 k^{2}}{2 n-3 k-1} ; \\
& A_{2}^{\prime}=\frac{k^{4}-k^{3}}{4 n^{2}-(12 k+4) n+9 k^{2}+6 k+1} \\
& A_{3}^{\prime}=\frac{k^{4}+5 k^{3}+2 k^{2}+6 k+12}{n-2 k} \\
& A_{4}^{\prime}=\frac{k^{6}+11 k^{5}+38 k^{4}+48 k^{3}+60 k^{2}+108 k+72}{4 n^{2}-16 k n+16 k^{2}}
\end{aligned}
$$

Since $n \geq k^{4}+5 k^{3}+2 k^{2}+8 k+12$, we obtain that $A_{1}^{\prime}+A_{2}^{\prime}+A_{3}^{\prime}-A_{4}^{\prime}<2$. Hence, the final inequality in (4.4) holds. 



\section{Chapter 5}

\section{Wiener and Harary indices on Hamilton-connected graphs}

In this chapter, we give some sufficient conditions for connected graphs with given minimum degree to be Hamilton-connected and traceable from every vertex in terms of Wiener index and Harary index. We also prove sufficient conditions on Harary index of $\bar{G}$ for $G$ to be Hamilton-connected and traceable from every vertex with given minimum degree.

\subsection{Introduction}

For definitions of Wiener index and Harary index, background and related results, we refer to Chapter 1 . And for more results about Wiener index and Harary index, we refer to $[14,17,18,43,48,61,62,67]$.

Then we start by recalling some of the notation and definitions of Chapter 3. We refrain from a long introduction. Instead, we present some important and necessary theorems and lemmas, and then put our main results and proofs in the next two sections.

For $n \geq 5$ and $1 \leq k \leq n / 2$, we define:

$$
S_{n}^{k}=K_{k} \vee\left(K_{n-(2 k-1)}+(k-1) K_{1}\right) \text { and } T_{n}^{k}=K_{2} \vee\left(K_{n-(k+1)}+K_{k-1}\right)
$$


The graph $S_{n}^{k}$ is obtained from $K_{n-k+1}$ and $(k-1) K_{1}$ by connecting all vertices of $(k-1) K_{1}$ to all vertices of a $k$-subset of $K_{n-k+1}$. The graph $T_{n}^{k}$ is obtained from $K_{n-k+1}$ and $K_{k+1}$ by identifying two vertices. Note that $S_{n}^{2}=T_{n}^{2}$. Denote by $\underline{S}_{n}^{k}$ and $\underline{T}_{n}^{k}$ the graphs obtained from $S_{n+1}^{k+1}$ and $T_{n+1}^{k+1}$, respectively, by deleting one vertex of degree $n$, i.e., for $0 \leq k \leq(n+1) / 2-1$,

$$
\underline{S}_{n}^{k}=K_{k} \vee\left(K_{n-2 k}+k K_{1}\right) \text { and } \underline{T}_{n}^{k}=K_{1} \vee\left(K_{n-k-1}+K_{k}\right) .
$$

Note that $\underline{S}_{n}^{1}=\underline{T}_{n}^{1}$.

We need the following result about relationship between Hamilton-connected graphs and graphs which is traceable from every vertex.

Lemma 5.1. (Exercise 18.1.6 on Page 474 of [6]). Let $G$ be a graph. Then $G$ is traceable from every vertex if and only if $G \vee K_{1}$ is Hamilton-connected.

Our proofs of main results depend on the following two theorems. The first one is due to [71]. One can see Chapter 4 for detailed proof.

Theorem 5.2. ( $[71]$ ) Let $G$ be a graph of order $n \geq 11 k$ with minimum degree $\delta(G) \geq k$, where $k \geq 2$. If

$$
e(G)>\left(\begin{array}{c}
n-k \\
2
\end{array}\right)+k(k+1),
$$

then $G$ is Hamilton-connected unless $G \subseteq S_{n}^{k}$ or $T_{n}^{k}$.

Theorem 5.3. Let $G$ be a graph of order $n \geq 11 k+10$ with minimum degree $\delta(G) \geq k$, where $k \geq 1$. If

$$
e(G)>\left(\begin{array}{c}
n-k-1 \\
2
\end{array}\right)+(k+1)^{2},
$$

then $G$ is traceable from every vertex unless $G \subseteq \underline{S}_{n}^{k}$ or $\underline{T}_{n}^{k}$.

Proof. Let $G^{\prime}=G \vee K_{1}$. Then we have $\left|V\left(G^{\prime}\right)\right|=n+1 \geq 11(k+1), \delta\left(G^{\prime}\right) \geq$ $k+1$ and

$$
e\left(G^{\prime}\right)=e(G)+n>\left(\begin{array}{c}
n-k-1 \\
2
\end{array}\right)+(k+1)^{2}+n=\left(\begin{array}{c}
n-k \\
2
\end{array}\right)+(k+1)(k+2) .
$$


By Theorem 5.2, $G^{\prime}$ is Hamilton-connected unless $G^{\prime} \subseteq S_{n+1}^{k+1}$ or $T_{n+1}^{k+1}$. From Lemma 5.1, we know that $G$ is traceable from every vertex if and only if $G^{\prime}$ is Hamilton-connected. Hence, $G$ is traceable from every vertex unless $G \subseteq \underline{S}_{n}^{k}$ or $\underline{T}_{n}^{k}$.

The following lemma show some observations from the definitions of Wiener index and Harary index.

Lemma 5.4. Let $G$ be a connected graph of order $n$ and $\bar{G}$ be its complement.

(1) If $G$ is connected, then $W(G) \geq n(n-1)-e(G)$, where the equality holds if and only if $\operatorname{diam}(G) \leq 2$;

(2) If $G$ is connected, then $H(G) \leq \frac{n}{4}(n-1)+\frac{1}{2} e(G)$, where the equality holds if and only if $\operatorname{diam}(G) \leq 2$;

(3) If $\bar{G}$ is connected, then $H(\bar{G}) \geq \frac{n(n-1)}{2}-\frac{n-2}{n-1} e(G)$, where the equality holds if and only if $\bar{G}=K_{n}$ or $P_{3}$.

Proof. (1)

$$
\begin{aligned}
W(G) & =\frac{1}{2} \sum_{i=1}^{n} \sum_{j=1}^{n} d\left(v_{i}, v_{j}\right) \\
& \geq \frac{1}{2} \sum_{i=1}^{n}\left(d\left(v_{i}\right)+2\left(n-1-d\left(v_{i}\right)\right)\right) \\
& =n(n-1)-\frac{1}{2} \sum_{i=1}^{n} d\left(v_{i}\right) \\
& =n(n-1)-e(G),
\end{aligned}
$$

where the equality holds if and only if $\operatorname{diam}(G) \leq 2$. 
(2)

$$
\begin{aligned}
H(G) & =\frac{1}{2} \sum_{i=1}^{n} \sum_{j=1}^{n} \frac{1}{d\left(v_{i}, v_{j}\right)} \\
& \leq \frac{1}{2} \sum_{i=1}^{n}\left(d\left(v_{i}\right)+\frac{1}{2}\left(n-1-d\left(v_{i}\right)\right)\right) \\
& =\frac{1}{4} n(n-1)+\frac{1}{4} \sum_{i=1}^{n} d\left(v_{i}\right) \\
& =\frac{n}{4}(n-1)+\frac{1}{2} e(G),
\end{aligned}
$$

where the equality holds if and only if $\operatorname{diam}(G) \leq 2$.

(3)

$$
\begin{aligned}
H(\bar{G}) & =\frac{1}{2} \sum_{i=1}^{n} \sum_{j=1}^{n} \frac{1}{d_{\bar{G}}\left(v_{i}, v_{j}\right)} \\
& \geq \frac{1}{2} \sum_{i=1}^{n}\left(d_{\bar{G}}\left(v_{i}\right)+\frac{1}{n-1}\left(n-1-d_{\bar{G}}\left(v_{i}\right)\right)\right) \\
& =\frac{1}{2} \sum_{i=1}^{n}\left(1+\frac{n-2}{n-1} d_{\bar{G}}\left(v_{i}\right)\right) \\
& =\frac{n}{2}+\frac{n-2}{2(n-1)} \sum_{i=1}^{n}\left(n-1-d_{G}\left(v_{i}\right)\right) \\
& =\frac{n(n-1)}{2}-\frac{n-2}{2(n-1)} \sum_{i=1}^{n} d_{G}\left(v_{i}\right) \\
& =\frac{n(n-1)}{2}-\frac{n-2}{n-1} e(G),
\end{aligned}
$$

where the equality holds if and only if $\bar{G}=K_{n}$ or $P_{3}$.

The proof is complete. 


\subsection{Wiener and Harary indices on $G$ for Hamilton- connectivity}

In this section, by imposing minimum degree, we will prove the results for graphs to be Hamilton-connected and traceable from every vertex in terms of Wiener index and Harary index.

Theorem 5.5. Let $G$ be a connected graph of order $n \geq 11 k$ with minimum degree $\delta(G) \geq k$, where $k \geq 2$. If $W(G) \leq W\left(S_{n}^{k}\right)$ or $H(G) \geq H\left(S_{n}^{k}\right)$, then $G$ is Hamilton-connected unless $G=S_{n}^{k}$.

Proof. Note that $\operatorname{diam}\left(S_{n}^{k}\right)=\operatorname{diam}\left(T_{n}^{k}\right)=2$. If $G_{0} \in\left\{S_{n}^{k}, T_{n}^{k}\right\}$, by (1) and (2) of Lemma 5.4, we have $W\left(G_{0}\right)=n(n-1)-e\left(G_{0}\right)$ and $H\left(G_{0}\right)=\frac{n}{4}(n-1)+$ 1 $\frac{1}{2} e\left(G_{0}\right)$.

If $W(G) \leq W\left(S_{n}^{k}\right)$, then by Lemma $5.4(1)$, we have

$$
\begin{aligned}
e(G) & \geq n(n-1)-W(G) \geq n(n-1)-W\left(S_{n}^{k}\right) \\
& =n(n-1)-\left[n(n-1)-e\left(S_{n}^{k}\right)\right]=e\left(S_{n}^{k}\right) \\
& =\left(\begin{array}{c}
n-k+1 \\
2
\end{array}\right)+k(k-1)>\left(\begin{array}{c}
n-k \\
2
\end{array}\right)+k(k+1),
\end{aligned}
$$

for $n>3 k$. If $H(G) \geq H\left(S_{n}^{k}\right)$, then by Lemma 5.4 (2), we have

$$
\begin{aligned}
e(G) & \geq 2 H(G)-\frac{n(n-1)}{2} \geq 2 H\left(S_{n}^{k}\right)-\frac{n(n-1)}{2} \\
& =e\left(S_{n}^{k}\right)=\left(\begin{array}{c}
n-k+1 \\
2
\end{array}\right)+k(k-1) \\
& >\left(\begin{array}{c}
n-k \\
2
\end{array}\right)+k(k+1),
\end{aligned}
$$

for $n>3 k$. By Theorem 5.2, we obtain that $G$ is Hamilton-connected unless $G \subseteq S_{n}^{k}$ or $T_{n}^{k}$.

If $G \varsubsetneqq S_{n}^{k}$, then $W(G)>W\left(S_{n}^{k}\right)$ and $H(G)<H\left(S_{n}^{k}\right)$, a contradiction. Note that $e\left(S_{n}^{k}\right)=\left(\begin{array}{c}n-k+1 \\ 2\end{array}\right)+k(k-1)$ and $e\left(T_{n}^{k}\right)=\left(\begin{array}{c}n-k+1 \\ 2\end{array}\right)+$ 
$\frac{(k+2)(k-1)}{2}$, then we have $e\left(S_{n}^{k}\right)>e\left(T_{n}^{k}\right)$ when $k \geq 3$ and $e\left(S_{n}^{k}\right)=e\left(T_{n}^{k}\right)$ when $k=2$. It follows that $W\left(S_{n}^{k}\right)<W\left(T_{n}^{k}\right)$ and $H\left(S_{n}^{k}\right)>H\left(T_{n}^{k}\right)$ when $k \geq 3$. Hence, if $G \subseteq T_{n}^{k}$ and $k \geq 3$, then $W(G) \geq W\left(T_{n}^{k}\right)>W\left(S_{n}^{k}\right)$ and $H(G) \leq H\left(T_{n}^{k}\right)<H\left(S_{n}^{k}\right)$, a contradiction. So $G=S_{n}^{k}$ for $k \geq 3$ or $k=2$ (in this case, $G=T_{n}^{2}=S_{n}^{2}$ ).

The proof is complete.

Theorem 5.6. Let $G$ be a connected graph of order $n \geq 11 k+10$ with minimum degree $\delta(G) \geq k$, where $k \geq 1$. If $W(G) \leq W\left(\underline{S}_{n}^{k}\right)$ or $H(G) \geq H\left(\underline{S}_{n}^{k}\right)$, then $G$ is traceable from every vertex unless $G=\underline{S}_{n}^{k}$.

Proof. Note that $\operatorname{diam}\left(\underline{S}_{n}^{k}\right)=\operatorname{diam}\left(\underline{T}_{n}^{k}\right)=2$. If $G_{0} \in\left\{\underline{S}_{n}^{k}, \underline{T}_{n}^{k}\right\}$, by (1) and (2) of Lemma 5.4, we have $W\left(G_{0}\right)=n(n-1)-e\left(G_{0}\right)$ and $H\left(G_{0}\right)=\frac{n}{4}(n-1)+$ $\frac{1}{2} e\left(G_{0}\right)$.

If $W(G) \leq W\left(\underline{S}_{n}^{k}\right)$, then by Lemma 5.4 (1), we have

$$
\begin{aligned}
e(G) & \geq n(n-1)-W(G) \geq n(n-1)-W\left(\underline{S}_{n}^{k}\right) \\
& =n(n-1)-\left[n(n-1)-e\left(\underline{S}_{n}^{k}\right)\right]=e\left(\underline{S}_{n}^{k}\right) \\
& =\left(\begin{array}{c}
n-k \\
2
\end{array}\right)+k^{2}>\left(\begin{array}{c}
n-k-1 \\
2
\end{array}\right)+(k+1)^{2},
\end{aligned}
$$

for $n>3 k+2$. If $H(G) \geq H\left(\underline{S}_{n}^{k}\right)$, then by Lemma 5.4 (2), we have

$$
\begin{aligned}
e(G) & \geq 2 H(G)-\frac{n(n-1)}{2} \geq 2 H\left(\underline{S}_{n}^{k}\right)-\frac{n(n-1)}{2} \\
& =e\left(\underline{S}_{n}^{k}\right)=\left(\begin{array}{c}
n-k \\
2
\end{array}\right)+k^{2} \\
& >\left(\begin{array}{c}
n-k-1 \\
2
\end{array}\right)+(k+1)^{2},
\end{aligned}
$$

for $n>3 k+2$. By Theorem 5.3, we obtain that $G$ is traceable from every vertex unless $G \subseteq \underline{S}_{n}^{k}$ or $\underline{T}_{n}^{k}$.

If $G \varsubsetneqq \underline{S}_{n}^{k}$, then $W(G)>W\left(\underline{S}_{n}^{k}\right)$ and $H(G)<H\left(\underline{S}_{n}^{k}\right)$, a contradiction. Note that $e\left(\underline{S}_{n}^{k}\right)=\left(\begin{array}{c}n-k \\ 2\end{array}\right)+k^{2}$ and $e\left(\underline{T}_{n}^{k}\right)=\left(\begin{array}{c}n-k \\ 2\end{array}\right)+k+\left(\begin{array}{l}k \\ 2\end{array}\right)$, then we have 
$e\left(\underline{S}_{n}^{k}\right)>e\left(\underline{T}_{n}^{k}\right)$ when $k \geq 2$ and $e\left(\underline{S}_{n}^{1}\right)=e\left(\underline{T}_{n}^{1}\right)$ when $k=1$. So if $G \subseteq \underline{T}_{n}^{k}$ and $k \geq 2$, then $W(G) \geq W\left(\underline{T}_{n}^{k}\right)>W\left(\underline{S}_{n}^{k}\right)$ and $H(G) \leq H\left(\underline{T}_{n}^{k}\right)<H\left(\underline{S}_{n}^{k}\right)$, a contradiction. It follows that $G=\underline{S}_{n}^{k}$ for $k \geq 2$ or $k=1$ (in this case, $\left.G=\underline{S}_{n}^{1}=\underline{T}_{n}^{1}\right)$.

The proof is complete.

\subsection{Harary index on $\bar{G}$ for the Hamilton-connectivity}

In this section, we will mainly present some sufficient conditions for the Hamilton-connectivity of a graph $G$ on Harary index of $\bar{G}$ with given minimum degree.

Theorem 5.7. Let $G$ be a graph of order $n \geq 11 k$ with minimum degree $\delta(G) \geq$ $k$, where $k \geq 2$, and $\bar{G}$ be its complement. If

$$
H(\bar{G})<\frac{(2 k+1) n^{2}-\left(3 k^{2}+7 k+1\right) n+6 k^{2}+6 k}{2(n-1)},
$$

then $G$ is Hamilton-connected.

Proof. Combining with (3) of Lemma 5.4, we have

$$
\frac{n(n-1)}{2}-\frac{n-2}{n-1} e(G) \leq H(\bar{G})<\frac{(2 k+1) n^{2}-\left(3 k^{2}+7 k+1\right) n+6 k^{2}+6 k}{2(n-1)} .
$$

Then $e(G)>\left(\begin{array}{c}n-k \\ 2\end{array}\right)+k(k+1)$. By Theorem 5.2, we obtain that $G$ is Hamilton-connected unless $G \subseteq S_{n}^{k}$ or $T_{n}^{k}$.

Recall that $\overline{S_{n}^{k}}=\overline{K_{k}}+\overline{K_{n-2 k+1}} \vee K_{k-1}$ and $\overline{T_{n}^{k}}=\overline{K_{2}}+\overline{K_{n-k-1}} \vee \overline{K_{k-1}}$. By simple calculation, we have

$$
H\left(\overline{S_{n}^{k}}\right)=\frac{n^{2}-3 n-2 k^{2}+4 k}{4}
$$




$$
\begin{aligned}
& =\frac{n^{3}-4 n^{2}-\left(2 k^{2}-4 k-3\right) n+2 k^{2}-4 k}{4(n-1)} \\
& >\frac{(2 k+1) n^{2}-\left(3 k^{2}+7 k+1\right) n+6 k^{2}+6 k}{2(n-1)},
\end{aligned}
$$

for $n \geq 11 k$ and $k \geq 2$. Furthermore, $H\left(\overline{T_{n}^{k}}\right)=\frac{n^{2}+(2 k-7) n-2 k^{2}+8}{4}$. Then we have $H\left(\overline{S_{n}^{2}}\right)=H\left(\overline{T_{n}^{2}}\right)$ for $k=2$. When $k \geq 3, n \geq 11 k$, we have $H\left(\overline{S_{n}^{k}}\right)-H\left(\overline{T_{n}^{k}}\right) \leq \frac{-22 k^{2}+48 k-8}{4}<0$, that is, $H\left(\overline{S_{n}^{k}}\right)<H\left(\overline{T_{n}^{k}}\right)$.

If $G \subseteq S_{n}^{k}$, then $\bar{G} \supseteq \overline{S_{n}^{k}}$. Hence

$$
H(\bar{G}) \geq H\left(\overline{S_{n}^{k}}\right)>\frac{(2 k+1) n^{2}-\left(3 k^{2}+7 k+1\right) n+6 k^{2}+6 k}{2(n-1)},
$$

a contradiction. If $G \subseteq T_{n}^{k}$, then $\bar{G} \supseteq \overline{T_{n}^{k}}$. Hence

$$
H(\bar{G}) \geq H\left(\overline{T_{n}^{k}}\right) \geq H\left(\overline{S_{n}^{k}}\right)>\frac{(2 k+1) n^{2}-\left(3 k^{2}+7 k+1\right) n+6 k^{2}+6 k}{2(n-1)},
$$

a contradiction.

The proof is complete.

Theorem 5.8. Let $G$ be a graph of order $n \geq 11 k+10$ with minimum degree $\delta(G) \geq k$, where $k \geq 1$, and $\bar{G}$ be its complement. If

$$
H(\bar{G})<\frac{(2 k+3) n^{2}-\left(3 k^{2}+11 k+9\right) n+6 k^{2}+14 k+8}{2(n-1)},
$$

then $G$ is traceable from every vertex.

Proof. Combining with (3) of Lemma 5.4, we have

$$
\begin{aligned}
\frac{n(n-1)}{2} & -\frac{n-2}{n-1} e(G) \leq H(\bar{G}) \\
& <\frac{(2 k+3) n^{2}-\left(3 k^{2}+11 k+9\right) n+6 k^{2}+14 k+8}{2(n-1)} .
\end{aligned}
$$


Then $e(G)>\left(\begin{array}{c}n-k-1 \\ 2\end{array}\right)+(k+1)^{2}$. By Theorem 5.3, we obtain that $G$ is traceable from every vertex unless $G \subseteq \underline{S}_{n}^{k}$ or $\underline{T}_{n}^{k}$.

Recall that $\overline{S_{n}^{k}}=\overline{K_{k}}+\overline{K_{n-2 k}} \vee K_{k}$ and $\overline{T_{n}^{k}}=\overline{K_{1}}+\overline{K_{n-k-1}} \vee \overline{K_{k}}$. By simple calculation, we have

$$
\begin{aligned}
H\left(\bar{S}_{n}^{k}\right) & =\frac{n^{2}-n-2 k^{2}}{4} \\
& =\frac{n^{3}-2 n^{2}-\left(2 k^{2}-1\right) n+2 k^{2}}{4(n-1)} \\
& >\frac{(2 k+3) n^{2}-\left(3 k^{2}+11 k+9\right) n+6 k^{2}+14 k+8}{2(n-1)},
\end{aligned}
$$

for $n \geq 11 k+10$ and $k \geq 1$. Furthermore,

$$
H\left(\underline{T}_{n}^{k}\right)=\frac{n^{2}+2 k n-3 n-2 k^{2}-2 k+2}{4} .
$$

Then we have $H\left(\underline{S}_{n}^{1}\right)=H\left(\bar{T}_{n}^{1}\right)$ for $k=1$. When $k \geq 2, n \geq 11 k+10$, we have $H\left(\bar{S}_{n}^{k}\right)-H\left(\underline{T}_{n}^{k}\right) \leq \frac{-22 k^{2}+4 k+18}{4}<0$, that is, $H\left(\underline{S}_{n}^{k}\right)<H\left(\bar{T}_{n}^{k}\right)$.

If $G \subseteq \underline{S}_{n}^{k}$, then $\bar{G} \supseteq \underline{S}_{n}^{k}$. Hence

$$
H(\bar{G}) \geq H\left(\bar{S}_{n}^{k}\right)>\frac{(2 k+3) n^{2}-\left(3 k^{2}+11 k+9\right) n+6 k^{2}+14 k+8}{2(n-1)},
$$

a contradiction. If $G \subseteq \underline{T}_{n}^{k}$, then $\bar{G} \supseteq \underline{T}_{n}^{k}$. Hence

$$
H(\bar{G}) \geq H\left(\bar{T}_{n}^{k}\right) \geq H\left(\bar{S}_{n}^{k}\right)>\frac{(2 k+3) n^{2}-\left(3 k^{2}+11 k+9\right) n+6 k^{2}+14 k+8}{2(n-1)},
$$

a contradiction.

The proof is complete. 



\section{Chapter 6}

\section{Hamiltonicity of $k$-connected graphs}

We have already presented and proved sufficient conditions on the spectral radius for hamiltonian properties of graphs with minimum degree at least $k$. In this chapter, we extend these results to $k$-connected, i.e., graphs with more than $k$ vertices that remain connected whenever fewer than $k$ vertices are removed. We present sufficient conditions on the spectral radius for the hamiltonicity and tracebility of such graphs.

\subsection{Introduction}

As we have seen throughout this thesis, since Dirac's result for hamiltonicity of graphs with minimum degree at least half the order of the graph, many researchers have focussed on obtaining more general results in different directions. Within algebraic graph theory, one way to obtain more widely applicable results is to lower the bound in conditions on $\rho(G)$ and $q(G)$. In this chapter, we follow this line of thought and mainly deal with $k$-connected graphs. To put our results in the right context, we first recall some previous results. 
For graphs with minimum degree $\delta(G) \geq k$, Nikiforov [52] gave the following hamiltonicity theorem involving the spectral radius of the graph.

Theorem 6.1 (Nikiforov [52]). Let $G$ be a graph of order $n$ with $\delta(G) \geq k$. If $k \geq 2, n \geq k^{3}+k+4$, and $\rho(G) \geq n-k-1$, then $G$ is hamiltonian unless $G=K_{1} \vee\left(K_{n-k-1}+K_{k}\right)$ or $G=K_{k} \vee\left(K_{n-2 k}+k K_{1}\right)$.

Recently, Ge and Ning [26] showed that the statement in the above theorem due to Nikiforov also holds for $k \geq 1$ and $n \geq \max \left\{\frac{1}{2} k^{3}+k+\frac{5}{2}, 6 k+5\right\}$.

For tracebility, Li and Ning [41] obtained the following result on spectral radius.

Theorem 6.2 (Li and Ning [41]). Let $G$ be a graph of order $n$ with $\delta(G) \geq k$. If $k \geq 0, n \geq \max \left\{6 k+10,\left(k^{2}+7 k+8\right) / 2\right\}$, and $\rho(G) \geq \rho\left(K_{k} \vee\left(K_{n-2 k-1}+\right.\right.$ $\left.\left.(k+1) K_{1}\right)\right)$, then $G$ is traceable unless $G=K_{k} \vee\left(K_{n-2 k-1}+(k+1) K_{1}\right)$.

In this chapter, we present new sufficient conditions based on the spectral radius for the hamiltonicity and traceability of $k$-connected graphs, which will extend Theorems 6.1 and 6.2. Before stating our results, we need to introduce some families of graphs.

For $n \geq 2 k+1$, we define $G_{n, k}^{1}=K_{k} \vee\left(K_{n-2 k}+k K_{1}\right)$.

For $n \geq 2 k+2$, we start with a graph consisting of vertex-disjoint graphs $k K_{1}$ and $K_{n-k-1}$, and an additional new vertex $v$, and we let $V\left(k K_{1}\right)=X$ and $V\left(K_{n-k-1}\right)=Y$. We choose $Y_{2} \subseteq Y$ with $\left|Y_{2}\right|=k$. Now, we let $G_{n, k}^{2}$ denote the graph obtained from this $k K_{1}+K_{n-k-1}+\{v\}$ by joining $X$ to $Y_{2}$, and $v$ to $Y_{2}$ and an arbitrary vertex in $X$ (See the graph sketched in the left part of Figure 6.1). We also define $G_{n, k}^{3}=K_{k} \vee\left(K_{n-2 k-1}+(k+1) K_{1}\right)$.

For $n \geq 2 k+3$, we start with a graph consisting of vertex-disjoint graphs $(k+1) K_{1}$ and $K_{n-k-1}$, and we let $V\left((k+1) K_{1}\right)=X$ and $V\left(K_{n-k-1}\right)=Y$. We choose $X_{1} \subseteq X$ with $\left|X_{1}\right|=k$, and $X_{2}=X \backslash X_{1}$, so with $\left|X_{2}\right|=1$, and $Y_{1} \subseteq Y$ with $\left|Y_{1}\right|=k$, and $Y_{2} \subseteq Y \backslash Y_{1}$ with $\left|Y_{2}\right|=1$. Now, we let $G_{n, k}^{4}$ denote the graph obtained from $(k+1) K_{1}+K_{n-k-1}$ by adding edges from $X$ to $Y_{1}$ and $X_{2}$ to $Y_{2}$ (See the graph sketched in the right part of Figure 6.1). We also define $G_{n, k}^{5}=K_{k+1} \vee\left(K_{n-2 k-2}+(k+1) K_{1}\right)$.

Additionally, we denote by $\underline{G}_{n, k}^{1}, \underline{G}_{n, k}^{2}, \underline{G}_{n, k}^{3}, \underline{G}_{n, k}^{4}, \underline{G}_{n, k}^{5}$ the graphs obtained from $G_{n+1, k+1}^{1}, G_{n+1, k+1}^{2}, G_{n+1, k+1}^{3}, G_{n+1, k+1}^{4}, G_{n+1, k+1}^{5}$ by deleting one vertex 
of degree $n$, respectively, i.e.,

$$
\begin{aligned}
& \underline{G}_{n, k}^{1}=K_{k} \vee\left(K_{n-2 k-1}+(k+1) K_{1}\right), \\
& \underline{G}_{n, k}^{3}=K_{k} \vee\left(K_{n-2 k-2}+(k+2) K_{1}\right), \\
& \underline{G}_{n, k}^{5}=K_{k+1} \vee\left(K_{n-2 k-3}+(k+2) K_{1}\right) .
\end{aligned}
$$

One easily checks that $\underline{G}_{n, k}^{2}$ is (again) the left graph in Figure 6.1 with $|X|=$ $k+1,|Y|=n-k-2$, and $\left|Y_{2}\right|=k$. Similarly, $\underline{G}_{n, k}^{4}$ is (again) the right graph in Figure 6.1 with $|X|=k+2,\left|X_{1}\right|=k+1,|Y|=n-k-2$, and $\left|Y_{1}\right|=k$.
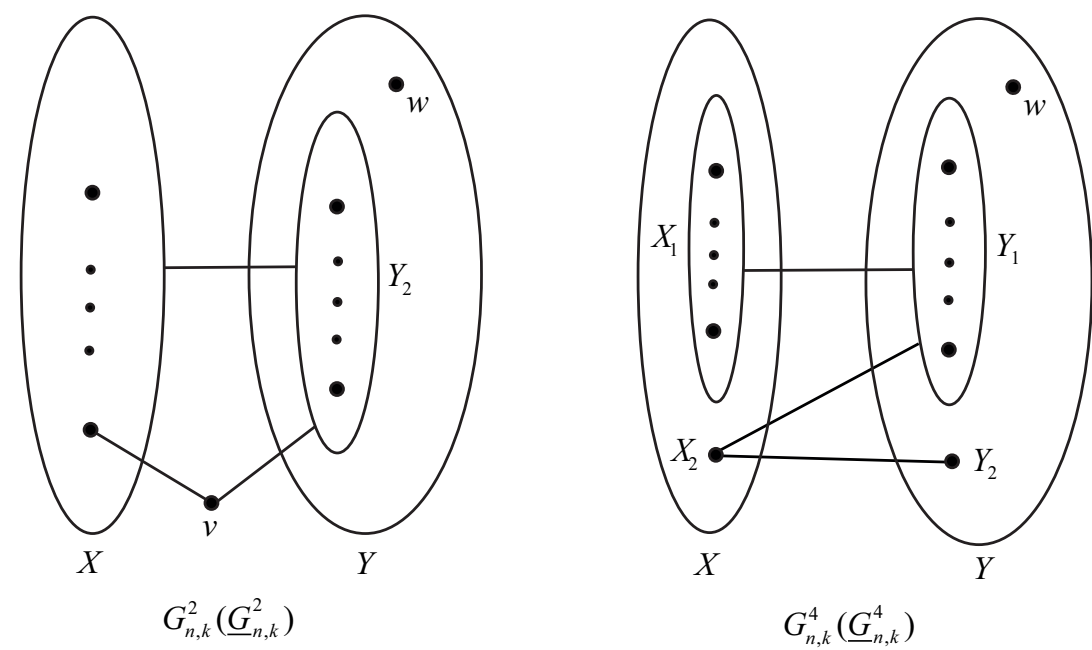

FIGURE 6.1: Some of the graphs appearing in our proofs.

In Section 6.3, we prove the following two main results.

Theorem 6.3. Let $G$ be a $k$-connected graph of order $n \geq k^{3}+k+2$, where $k \geq 2$. If $\rho(G)>n-k-1-\frac{1}{n}$, then $G$ is hamiltonian unless $G=G_{n, k}^{1}$.

Theorem 6.4. Let $G$ be a $k$-connected graph of order $n \geq k^{3}+k^{2}+k+3$, where $k \geq 1$. If $\rho(G)>n-k-2-\frac{1}{n}$, then $G$ is traceable unless $G=\underline{G}_{n, k}^{1}$.

Obviously, in some sense, Theorems 6.3 and 6.4 improve Theorems 6.1 and 6.2 , respectively. 
The rest of this chapter is organized as follows. In Section 6.2, we will recall two known result as lemmas that will be used in our proofs. In Section 6.3, we present several other necessary lemmas, together with our proofs of Theorems 6.3 and 6.4 .

\subsection{Two useful lemmas}

We have already mentioned the concept of closure that was introduced by Bondy and Chvátal [5] in previous chapters, but repeat it here for convenience and later reference. The $k$-closure of a graph $G$ is denoted by $c l_{k}(G)$, and can be obtained from $G$ by recursively joining nonadjacent vertices with degree sum at least $k$ until no such pair remains in the current graph. When $k=n$, we use $\operatorname{cl}(G)$ instead of $c l_{n}(G)$. A graph is called closed if $G=\operatorname{cl}(G)$.

Lemma 6.5 (Bondy and Chvátal [5]). A graph $G$ is hamiltonian if and only if $\operatorname{cl}(G)$ is hamiltonian.

The next lemma is one of the generalizations of Dirac's theorem due to Chvátal and Erdős [11].

Lemma 6.6 (Chvátal and Erdős [11]). A graph $G$ with at least three vertices is hamiltonian if $\kappa(G) \geq \alpha(G)$.

Recall that $\kappa(G)$ and $\alpha(G)$ denote the (vertex) connectivity and independence number of $G$, respectively.

\subsection{Main results and proofs}

From Lemma 1 in [41], we have the following lemma.

Lemma 6.7. Let $G$ be a closed graph of order $n \geq 6 k+11$, where $k \geq 0$. If $e(G)>\left(\begin{array}{c}n-k-2 \\ 2\end{array}\right)+(k+2)^{2}$, then $\omega(G) \geq n-k-1$.

As before, here $\omega(G)$ denotes the cardinality of a largest clique in $G$. Using the above lemma, we can prove the following structure theorem. In the proof, we use $P_{u v} \bigsqcup P_{w z}$ (again) to denote a path obtained from two vertex-disjoint paths $P_{u v}$ and $P_{w z}$ by joining $v$ and $w$ with an edge. 
Theorem 6.8. Let $G$ be a $k$-connected graph of order $n \geq 6 k+11$, where $k \geq 2$. If $e(G)>\left(\begin{array}{c}n-k-2 \\ 2\end{array}\right)+(k+2)^{2}$, then $G$ is hamiltonian unless $\operatorname{cl}(G) \in$ $\left\{G_{n, k}^{1}, G_{n, k}^{2}, G_{n, k}^{3}, G_{n, k}^{4}, G_{n, k}^{5}\right\}$.

Proof. Let $H=\operatorname{cl}(G)$. If $H$ is hamiltonian, then so is $G$ by Lemma 6.5. Now, we assume that $H$ is not hamiltonian. Note that $H$ is $k$-connected and $e(H) \geq$ $e(G)$. By Lemma 6.7, we have $\omega(H) \geq n-k-1$. We claim that $\omega(H) \leq n-k$. In fact, if $\omega(H) \geq n-k+1$, then $\alpha(H) \leq k$. Then, by Lemma 6.6, $H$ is hamiltonian, which contradicts our assumption. Note that $\delta(G) \geq \kappa(G) \geq k$. Next we distinguish two cases.

Case 1. $\omega(H)=n-k$.

In this case, we have $\alpha(H)=k+1$. Set $V(H)=X \cup Y$, where $X \cap Y=\emptyset$, $|X|=k,|Y|=n-k, H[Y]=K_{n-k}$ and $X$ together with one vertex in $Y$, say $w$, is a maximum independent set in $H$. Note that $\delta(H) \geq \kappa(H) \geq k$. From the fact that $H$ is closed and $d_{H}(w)=n-k-1$, we have $d_{H}(x)=k$ for every $x \in X$. Let $F \subseteq Y$ be the neighbors of $X$ in $Y$. Obviously $w \notin F$. Then every vertex in $F$ is adjacent to every vertex in $X$, and hence $|F|=k$. So $H=K_{k} \vee\left(K_{n-2 k}+k K_{1}\right)=G_{n, k}^{1}$.

Case 2. $\omega(H)=n-k-1$.

In this case, we have $\alpha(H)=k+1$ or $k+2$. We discuss these two subcases separately.

Subcase $2.1 \alpha(H)=k+1$.

We first consider the situation that $V(H)$ can be divided into two subsets, i.e., $V(H)=X \cup Y$, such that $X \cap Y=\emptyset,|X|=k+1,|Y|=n-k-1$, $H[X]=(k+1) K_{1}$ and $H[Y]=K_{n-k-1}$. In this situation, each vertex of $Y$ must be adjacent to some vertex in $X$; otherwise $\alpha(H)=k+2$. Set $Y=Y_{1} \cup Y_{2}$ such that each vertex of $Y_{1}$ has only one neighbor in $X$ and each vertex of $Y_{2}$ has at least two neighbors in $X$. Recall that $H$ is closed. If $Y_{1}=\emptyset$, then $H=K_{n-k-1} \vee(k+1) K_{1}$, which is hamiltonian, a contradiction. If $Y_{2}=\emptyset$, then obviously $H$ has a Hamilton cycle, a contradiction. Next, let $Y_{1} \neq \emptyset$ and $Y_{2} \neq \emptyset$. Then $d_{H}(y)=n-k-1$ for $y \in Y_{1}$, and $Y_{2}$ is adjacent to $X$ since $d_{H}(y) \geq n-k$ for $y \in Y_{2}$. Denote by $X_{1}$ the neighbor set of $Y_{1}$ in $X$, and let 
$X_{2}=X \backslash X_{1}$. If $\left|X_{1}\right| \geq 2$, then $d_{H}(x)=k$ for $x \in X_{1}$; otherwise $Y_{1}$ will be adjacent to $X_{1}$, and each vertex of $Y_{1}$ has more than one neighbor in $X$. Then we have $\left|Y_{2}\right| \leq k-1$ and hence $d_{H}(x) \leq k-1$ for $x \in X_{2}$, a contradiction. If $\left|X_{1}\right|=1$, then the vertex $x \in X_{1}$ is adjacent to $Y$, which means $\omega(H)=n-k$, a contradiction.

The other situation is that $V(H)=X \cup Y \cup\{v\}$, such that $X \cap Y \cap\{v\}=\emptyset$, $|X|=k,|Y|=n-k-1, H[Y]=K_{n-k-1}$, and $X$ together with one vertex of $Y$, say $w$, is a maximum independent set in $H$. We use the same notations as in the first situation. We deduce that $v$ is adjacent to $Y_{2}$, and that $v$ must be adjacent to at least one vertex in $X$; otherwise $\alpha(H)=k+2$.

We first assume $v$ is also adjacent to $Y \backslash\left\{Y_{1} \cup Y_{2}\right\}$, that is to say, all possible $w$ have degree $n-k-1$. Then $d_{H}(x)=k$ for all $x \in X$. In this case, we have $Y_{1} \neq \emptyset$; otherwise $v$ is adjacent to $Y$ and $\omega(H)=n-k$, and hence $X_{1} \neq \emptyset$. If $X_{2} \neq \emptyset$, then $\left|Y_{2}\right|=k-1, v$ is adjacent to $X_{2}$, and each vertex of $X_{1}$ has exactly one neighbor in $Y_{1}$. Hence $\left|Y_{1}\right|=\left|X_{1}\right| \leq k-1$. Suppose $\left|X_{1}\right|=a \geq 1$ and $\left|X_{2}\right|=b \geq 1$, where $a+b=k$. We use $G_{1}$ to denote the graph when $v$ has no neighbors in $Y \backslash\left(Y_{1} \cup Y_{2}\right)$ (See Figure 6.2). Obviously, we have $G_{1} \subseteq H$. Label the vertices of $G_{1}$ as $x_{11}, \ldots, x_{1 a} ; x_{21}, \ldots, x_{2 b} ; y_{11}, \ldots, y_{1 a}$; $y_{21}, \ldots, y_{2 a}, y_{31}, \ldots, y_{3, b-1} ; y_{41}, \ldots, y_{4 c}$ (See Figure 6.2), where $c=n-2 k-$ $a$. Let $Q_{1}=y_{11} x_{11} y_{21} x_{12} y_{12} y_{13} x_{13}, Q_{2}=y_{22} x_{14} y_{23} x_{15} \cdots y_{2, a-2} x_{1 a}, Q_{3}=$ $y_{31} x_{21} \cdots y_{3, b-1} x_{2, b-1} v x_{2 b}$ and $Q_{4}=y_{2, a-1} y_{2 a} y_{41} \cdots y_{4 c} y_{11}$. Then $\bigsqcup_{i=1}^{4} Q_{i}$ is a Hamilton cycle. Hence $H$ is also hamiltonian, a contradiction. If $X_{2}=\emptyset$, then $\left|Y_{2}\right| \leq k-2$. Indeed, if $\left|Y_{2}\right| \geq k-1$, then either $v$ has no neighbors in $X_{1}$ or the vertices in $X_{1}$ have degree more than $k$, both leading to a contradiction. Suppose $\left|Y_{2}\right|=t$. Then each $x \in X_{1} \backslash N_{H\left[X_{1}\right]}(v)$ has $k-t$ neighbors in $Y_{1}$, and each $x \in N_{H\left[X_{1}\right]}(v)$ has $k-t-1$ neighbors in $Y_{1}$. When $t=k-2$, we use $G_{2}$ to denote the graph if $v$ has no neighbors in $Y \backslash\left(Y_{1} \cup Y_{2}\right.$ ) (See Figure 6.2). Obviously, $G_{2} \subseteq H$. Label the vertices of $G_{2}$ as $x_{11}, \ldots, x_{1 a}$; $x_{21}, \ldots, x_{2 b} ; y_{11}^{1}, y_{11}^{2}, \ldots, y_{1 a}^{1}, y_{1 a}^{2} ; y_{21}^{1}, \ldots, y_{2 b}^{1} ; y_{31}, \ldots, y_{3, b-2}, y_{41}, \ldots, y_{4 a}$; $y_{51}, \ldots, y_{5 c}$, where $a \geq 1, b \geq 1, a+b=k$ and $c=n-3 k-a+1$. Let $Q_{1}=$ $y_{11}^{1} x_{11} y_{11}^{2} \cdots y_{1 a}^{1} x_{1 a} y_{1 a}^{2}, Q_{2}=y_{21}^{1} x_{21} y_{31} \cdots x_{2, b-2} y_{3, b-2} x_{2, b-1} v x_{2 b} y_{2 b}^{1}$ and $Q_{3}=y_{22}^{1} \cdots y_{2, b-1}^{1} y_{41} \cdots y_{4 a} y_{51} \cdots y_{5 c} y_{11}^{1}$ (See Figure 6.2). Then $\bigsqcup_{i=1}^{3} Q_{i}$ is a Hamilton cycle of $G_{2}$. Hence $H$ is also hamiltonian, a contradiction. When $t \leq k-3$, we have that each vertex of $X_{1}$ has at least two neighbors in $Y_{1}$. 
Obviously, $H$ has a Hamilton cycle, a contradiction.

Next, we assume that there exists a vertex $w$ such that its degree is $n-$ $k-2$, i.e., $v$ is not adjacent to $w$. In this case, $d_{H}(x)=k$ or $k+1$ for $x \in X$.
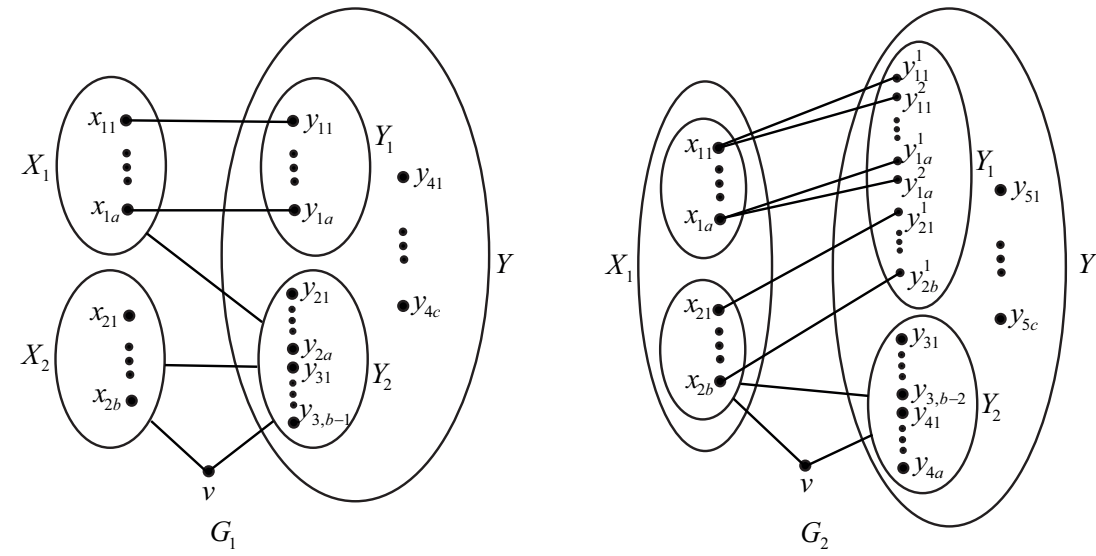

FIgURE 6.2: The graphs $G_{1}$ and $G_{2}$ from the proof.

If $Y_{1}=\emptyset$, then $X_{1}=\emptyset$. If $d_{H}(x)=k+1$ for all $x \in X_{2}$, then $\left|Y_{2}\right|=k$, and $v$ is adjacent to $X_{2}$. Then we can easily find a Hamilton cycle in $H$, a contradiction. If $d_{H}(x)=k$ for all $x \in X_{2}$, then $\left|Y_{2}\right|=k-1(k \geq 2)$ and again $v$ is adjacent to $X$. Hence $d_{H}(v) \geq 2 k-1$. We see that $v$ must have at least one neighbor in $Y \backslash Y_{2}$; otherwise $\kappa(H)=k-1$. Now $d_{H}(v) \geq 2 k$ and $d_{H}(v)+d_{H}(w) \geq 2 k+n-k-2=n+k-2 \geq n$, which means $v$ is adjacent to $Y \backslash Y_{2}$, a contradiction. If some vertices in $X_{2}$ have degree $k$ and some vertices in $X_{2}$ have degree $k+1$, then $\left|Y_{2}\right|=k$, and the vertices in $X_{2}$ with degree $k+1$ are adjacent to $v$. In this case, if $v$ has a neighbor in $Y \backslash Y_{2}$ or $v$ has at least two neighbors in $X$, then $H$ is obviously hamiltonian, a contradiction. If $v$ has no neighbor in $Y \backslash Y_{2}$ and has only one neighbor in $X$, then $H=G_{n, k}^{2}$.

If $Y_{2}=\emptyset$, it is easy to see that $H$ is hamiltonian, a contradiction.

If $Y_{1} \neq \emptyset$ and $Y_{2} \neq \emptyset$, when $X_{2} \neq \emptyset$, then $d_{H}(x)=k$ for $x \in X_{2}$ since the vertices in $X_{2}$ have no neighbors in $Y_{1}$. If $\left|Y_{2}\right|=k$, then on the one hand, there is no neighbor of $v$ in $X_{2}$; otherwise its neighbor will be adjacent to $Y_{1}$. On the other hand, $v$ cannot be adjacent to any $x \in X_{1}$; otherwise $d_{H}(x)=k+2$, a contradiction. Therefore, $\left|Y_{2}\right|=k-1$ and hence, $X_{2}$ is adjacent to $v$. In this case, every vertex in $X_{1}$ has a one-to-one neighbor in $Y_{1}$. Also, $v$ must 
be adjacent to at least one vertex in $Y \backslash\left(Y_{1} \cup Y_{2}\right)$; otherwise $Y_{2}$ will be a cut set of $H$. Now we conclude that $G_{1} \subseteq H$. By the former proof, we obtain that $H$ is hamiltonian, a contradiction. When $X_{2}=\emptyset$, then for $x \in X_{1}$, we have $d_{H}(x)=k$. We can obtain that $\left|Y_{2}\right| \leq k-2$. Let $\left|Y_{2}\right|=t$. Denote by $X_{11}=N_{H\left[X_{1}\right]}(v)$ and $X_{12}=X_{1} \backslash X_{11}$. Then the vertices of $X_{11}$ have $k-t-1$ neighbors in $Y_{1}$, and the vertices of $X_{12}$ have $k-t$ neighbors in $Y_{1}$. Obviously, when $t \leq k-3$, every vertex in $X_{1}$ has more than two neighbors in $Y_{1}$, and it is easy to find a Hamilton cycle in $H$, a contradiction. When $t=k-2$, we obtain that $G_{2} \subseteq H$. Then, by the former proof, $H$ is also hamiltonian, a contradiction.

Subcase $2.2 \alpha(H)=k+2$.

Set $V(H)=X \cup Y$, where $X \cap Y=\emptyset,|X|=k+1,|Y|=n-k-1, H[Y]=$ $K_{n-k-1}$, and $X$ together with one vertex in $Y$, say $w$, is a maximum independent set in $H$. Since $d_{H}(w)=n-k-2$, we know that $d_{H}(x)=k$ or $k+1$ for $x \in X$. Let $X_{1} \subseteq X$ be the set, the vertices of which have degree $k$, and let $X_{2}=X \backslash X_{1}$ be the set each vertex of which has degree $k+1$. Denote by $Y_{1}$ the neighbors of $X_{1}$ in $Y$, and $Y_{2}=N_{H[Y]}\left(X_{2}\right) \backslash Y_{1}$.

The first situation we consider is that $X_{1}=\emptyset$. Then $X$ is adjacent to $Y_{2}$, and $\left|Y_{2}\right|=k+1$. Hence $H=K_{k+1} \vee\left(K_{n-2 k-2}+(k+1) K_{1}\right)=G_{n, k}^{5}$.

The second situation we consider is that $X_{1} \neq \emptyset$ and $X_{2} \neq \emptyset$. Firstly, $Y_{1}$ is adjacent to $X_{2}$ since $d_{H}(y) \geq n-k-1$ for $y \in Y_{1}$. So, now every vertex of $Y_{1}$ has at least two neighbors in $X$, and hence $d_{H}(y) \geq n-k$ for $y \in Y_{1}$. Then every vertex in $X_{1}$ is adjacent to every vertex in $Y_{1}$, and hence $\left|Y_{1}\right|=k$. Then $x \in X_{2}$ has a one-to-one neighbor in $Y_{2}$, hence $\left|X_{2}\right|=\left|Y_{2}\right|$. When $\left|X_{2}\right|=\left|Y_{2}\right|=$ 2 , label the vertices of $H$ as $x_{1}, x_{2}, \ldots, x_{k+1} ; y_{0}, y_{1}, \ldots, y_{k+1} ; y_{11}, y_{12}, \ldots, y_{1, n-2 k-3}$ (See Figure 6.3 (1)). Let $Q_{1}=y_{0} x_{k} y_{k} y_{11} y_{12} \ldots y_{1, n-2 k-3} y_{k+1} x_{k+1}, Q_{2}=$ $y_{1} x_{1} y_{2} x_{2} \ldots y_{k-1} x_{k-1} y_{0}$. Obviously $P=Q_{1} \bigsqcup Q_{2}$ is a Hamilton cycle, a contradiction. If $\left|X_{2}\right|=\left|Y_{2}\right| \geq 3$, label the vertices of $H$ as $x_{11}, \ldots, x_{1 a} ; x_{21}$, $\ldots, x_{2 b} ; y_{11}, \ldots, y_{1 a}, y_{1, a+1}, \ldots, y_{1, b-1} ; y_{21}, \ldots, y_{2 b} ; y_{31}, \ldots, y_{3, n-2 k-b-1}$ (See Figure 6.3 (2)), where $\left|X_{1}\right|=a,\left|X_{2}\right|=b \geq 3, a+b=k+1$. Let $Q_{1}=$ $y_{11} x_{11} \ldots y_{1 a} x_{1 a}$. If $b$ is even, let $Q_{2 i}=y_{1, a+i} x_{2,2 i-1} y_{2,2 i-1} y_{2,2 i} x_{2,2 i}(1 \leq i \leq$ $\frac{b}{2}$ ) and $Q_{3}=y_{1, \frac{b}{2}+1} \ldots y_{1, b-1} y_{31} \ldots y_{3, n-2 k-b-1}$. Then we obtain a Hamilton cycle $Q_{1} \bigsqcup\left(\bigsqcup_{i=1}^{b / 2} Q_{2 i}\right) \bigsqcup Q_{3} y_{11}$, a contradiction. If $b$ is odd, let $Q_{3}^{\prime}=$ 
$y_{1, \frac{b-1}{2}+1} x_{2 b} y_{2 b} y_{1, \frac{b-1}{2}+2} \ldots y_{1, b-1} y_{31} \ldots y_{3, n-2 k-b-1}$. Then we obtain a Hamilton cycle $Q_{1} \bigsqcup\left(\bigsqcup_{i=1}^{(b-1) / 2} Q_{2 i}\right) \bigsqcup Q_{3}^{\prime} y_{11}$, a contradiction. When $\left|X_{2}\right|=\left|Y_{2}\right|=$ $1, H=G_{n, k}^{4}$.

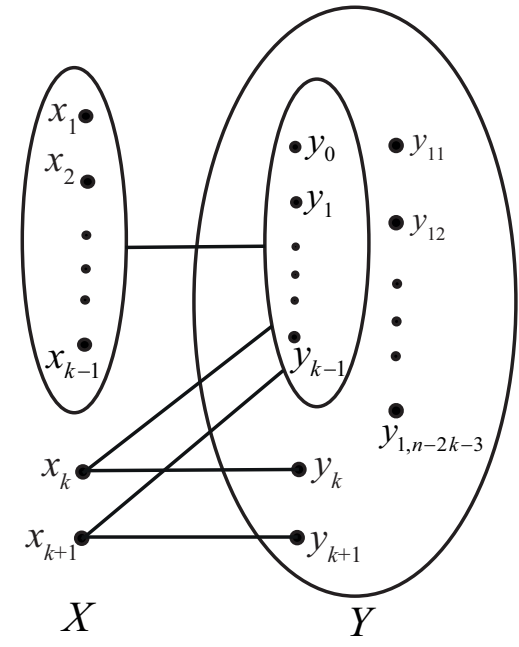

(1)

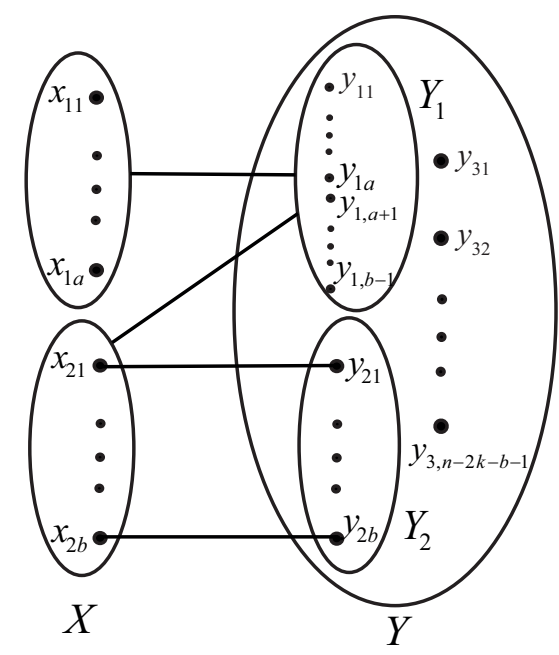

(2)

FIgURE 6.3: The graph $H$ from the proof.

The third situation we consider is that $X_{2}=\emptyset$. Then $Y_{2}=\emptyset$. Set $Y_{1}=$ $Y_{11} \cup Y_{12}$, where $Y_{11}$ is the set of vertices with only one neighbor in $X$, and $Y_{12}$ is the set of vertices with at least two neighbors in $X$. Let $Y_{3}=Y \backslash Y_{1}$, i.e., $Y_{3}$ is the set of vertices with no neighbor in $X$. Obviously $w \in Y_{3}, d_{H}(y)=n-k-1$ for $y \in Y_{11}$ and $d_{H}(y) \geq n-k$ for $y \in Y_{12}$.

If $Y_{11}=\emptyset$, then it is easy to see that $X$ is adjacent to $Y_{12},\left|Y_{12}\right|=k$, and hence $H=K_{k} \vee\left(K_{n-2 k-1}+(k+1) K_{1}\right)=G_{n, k}^{3}$.

If $Y_{12}=\emptyset$, then obviously $H$ is hamiltonian, a contradiction.

If $Y_{11} \neq \emptyset$ and $Y_{12} \neq \emptyset$, then $\left|Y_{11}\right|+\left|Y_{12}\right| \geq k+1$, and $X$ is adjacent to $Y_{12}$. Let $\left|Y_{11}\right|=a$. Then $1 \leq a \leq k+1$. If $1 \leq a \leq k$, then $\left|Y_{12}\right|=k$ and each vertex in $N_{H[X]}\left(Y_{11}\right)$ has degree $k+1$, a contradiction. So $a=k+1$, i.e., every vertex in $X$ has a one-to-one neighbor in $Y_{11}$, which leads to $\left|Y_{12}\right|=k-1$. If $k+1$ is even, then label the vertices of $H$ as $x_{1}, x_{2}, \ldots, x_{k+1} ; y_{11}, y_{12}, \ldots, y_{1, k+1} ; y_{21}$, $\ldots, y_{2, \frac{k+1}{2}}, y_{2, \frac{k+3}{2}}, \ldots, y_{2, k-1} ; y_{31}, \ldots, y_{3 b}$ (See Figure 6.4 (3)), where $b=n-$ 
$3 k-1$. Let $Q_{i}=y_{1,2 i-1} x_{2 i-1} y_{2, i} x_{2 i} y_{1,2 i}\left(i=1,2, \ldots, \frac{k+1}{2}\right)$ and let $Q=$ $y_{2, \frac{k+3}{2}} \ldots y_{2, k-1} y_{31} y_{32} \ldots y_{3 b}$. Then $\left(\bigsqcup_{i=1}^{\frac{k+1}{2}} Q_{i}\right) \bigsqcup Q y_{11}$ is a Hamilton cycle in $H$, a contradiction.

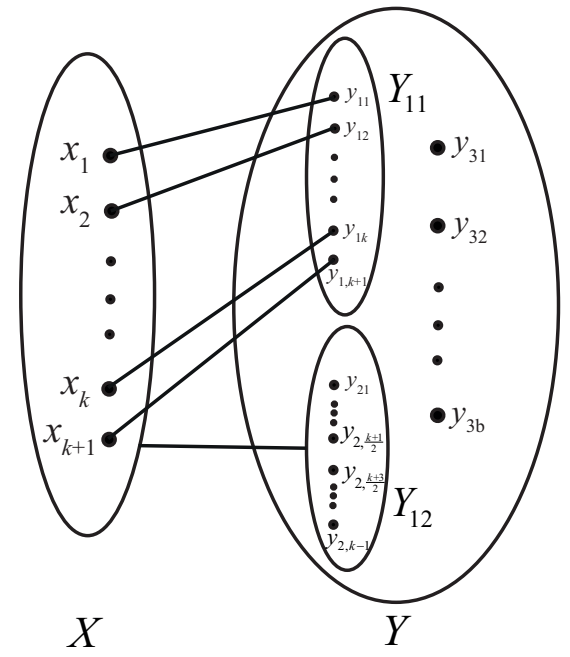

(3)

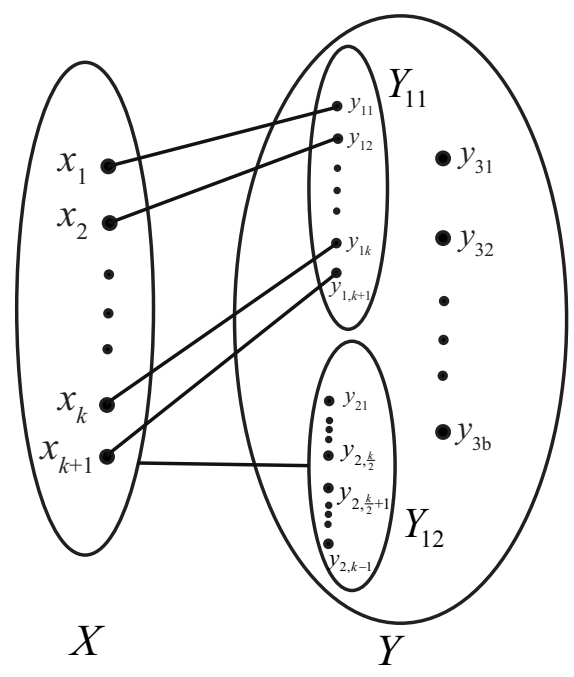

(4)

Figure 6.4: The graph $H$ from the proof.

If $k+1$ is odd, label the vertices of $H$ as $x_{1}, x_{2}, \ldots, x_{k+1} ; y_{11}, y_{12}, \ldots, y_{1, k+1}$; $y_{21}, \ldots, y_{2, \frac{k}{2}} y_{2, \frac{k}{2}+1}, \ldots, y_{2, k-1} ; y_{31}, \ldots, y_{3 b}$ (See Figure 6.4 (4)), where $b=$ $n-3 k-1$. Let $Q^{\prime}=y_{1, k+1} x_{1, k+1} y_{2, \frac{k}{2}+1} y_{2, \frac{k}{2}+2} \ldots y_{2, k-1} y_{31} y_{32} \ldots y_{3 b}$. Then $\left(\bigsqcup_{i=1}^{\frac{k}{2}} Q_{i}\right) \bigsqcup Q^{\prime} y_{11}$ is a Hamilton cycle in $H$, a contradiction.

This completes the proof of Theorem 6.8 .

In order to prove Theorem 6.3, we also need the following result on the spectral radius.

Lemma 6.9. Let $G$ be a graph of order $n$ with $\delta(G) \geq k$, where $n \geq k^{3}+k+2$ and $k \geq 2$.

(i) If $G$ is a proper subgraph of $G_{n, k}^{1}$, then $\rho(G)<n-k-1-\frac{1}{n}$.

(ii) If $G \in\left\{G_{n, k}^{2}, G_{n, k}^{3}, G_{n, k}^{4}, G_{n, k}^{5}\right\}$, then $\rho(G)<n-k-1-\frac{1}{n}$. 
Proof. (i) For $G_{n, k}^{1}=K_{k} \vee\left(K_{n-2 k}+k K_{1}\right)$, let $X$ be the set of vertices with degree $k$, let $Y$ be the set of neighbors of $X$, and let $Z$ be the remaining set of $n-2 k$ vertices. Assume that $G$ is a proper subgraph obtained from $G_{n, k}^{1}$ by deleting one edge $u v \in E\left(G_{n, k}^{1}\right)$. According to the proof of Theorem 1.6 in [52], we know that there are three possible cases, and that the case with $u, v \in Z$ yields the largest spectral radius. It is sufficient to prove $\rho(G)<$ $n-k-1-\frac{1}{n}$ for this case.

Let us consider the following partition $\pi$ of $V(G)$ in $X_{1}=X, X_{2}=Y, X_{3}=$ $Z \backslash\{u, v\}$, and $X_{4}=\{u, v\}$. It is easy to check that this partition is equitable, and that the corresponding adjacency matrix of the quotient matrix of $G$ is as follows:

$$
A(G / \pi)=\left(\begin{array}{cccc}
0 & k & 0 & 0 \\
k & k-1 & n-2 k-2 & 2 \\
0 & k & n-2 k-3 & 2 \\
0 & k & n-2 k-2 & 0
\end{array}\right) .
$$

By using the Laplace expansion for the calculation of the determinant, we obtain the following characteristic polynomial of $A(G / \pi)$.

$$
\begin{aligned}
f_{1}(x) & =x^{4}+(k-n+4) x^{3}+\left(-k^{2}+3 k-3 n+7\right) x^{2} \\
& +\left(2 k-2 n+k^{2} n-3 k^{2}-2 k^{3}+4\right) x+2 k^{2} n-4 k^{2}-4 k^{3} .
\end{aligned}
$$

We can obtain the following derivatives of $f_{1}(x)$ with respect to $x$, by standard analysis.

$$
\begin{aligned}
f_{1}^{\prime}(x)= & 4 x^{3}+3(k-n+4) x^{2}+2\left(-k^{2}+3 k-3 n+7\right) x+2 k-2 n \\
& +k^{2} n-3 k^{2}-2 k^{3}+4, \\
f_{1}^{(2)}(x)= & 12 x^{2}+6(k-n+4) x+2\left(-k^{2}+3 k-3 n+7\right), \\
f_{1}^{(3)}(x)= & 24 x+6(k-n+4), \\
f_{1}^{(4)}(x)= & 24 .
\end{aligned}
$$

By substitution and simple but tedious calculations, we obtain 


$$
\begin{aligned}
f_{1}\left(n-k-1-\frac{1}{n}\right)= & n^{2}-\left(k^{3}+k+2\right) n+\frac{k^{3}+k^{2}-3 k+2}{n}+\frac{2 k^{2}-2}{n^{2}} \\
& +\frac{3 k}{n^{3}}+\frac{1}{n^{4}}+k^{4}-k^{3}-2 k^{2}+2 k \\
> & n^{2}-\left(k^{3}+k+2\right) n+k^{4}-k^{3}-2 k^{2}+2 k \\
= & g_{1}(n) \geq 0,
\end{aligned}
$$

where $g_{1}(x)=x^{2}-\left(k^{3}+k+2\right) x+k^{4}-k^{3}-2 k^{2}+2 k$. For the last inequality, obviously, the function $g_{1}(x)$ is strictly increasing when $x \geq k^{3}+k+2$. Using $n \geq k^{3}+k+2$, we obtain $g_{1}(n) \geq g_{1}\left(k^{3}+k+2\right)=k^{4}-k^{3}-2 k^{2}+2 k \geq 0$.

$$
\begin{aligned}
f_{1}^{\prime}\left(n-k-1-\frac{1}{n}\right)= & n^{3}-3 k n^{2}+\left(2 k^{2}-3\right) n-\frac{4 k^{2}-7}{n}-\frac{9 k}{n^{2}}-\frac{4}{n^{3}}-k^{3} \\
& -k^{2}+9 k-2 \\
= & g_{2}(n) \geq g_{2}\left(k^{3}+k+2\right) \\
= & k^{9}+6 k^{6}-k^{5}+8 k^{3}-3 k^{2}+6 k-\frac{4 k^{2}-7}{k^{3}+k+2} \\
& -\frac{9 k}{\left(k^{3}+k+2\right)^{2}}-\frac{4}{\left(k^{3}+k+2\right)^{3}} \\
\geq & k^{9}+\left(6 k^{6}-k^{5}\right)+\left(8 k^{3}-3 k^{2}\right)+6 k-\frac{3}{4}-\frac{1}{8}-\frac{1}{432} \\
> & 0,
\end{aligned}
$$

where $g_{2}(x)=x^{3}-3 k x^{2}+\left(2 k^{2}-3\right) x-\frac{4 k^{2}-7}{x}-\frac{9 k}{x^{2}}-\frac{4}{x^{3}}-k^{3}-k^{2}+9 k-2$. For the first inequality, the first derivative of $g_{2}(x)$ is $g_{2}^{\prime}(x)=3 x^{2}-6 k x+$ $2 k^{2}-3+\frac{4 k^{2}-7}{x^{2}}+\frac{18 k}{x^{3}}+\frac{12}{x^{4}}$. Since $g_{2}^{\prime}(x) \geq g_{2}^{\prime}\left(k^{3}+k+2\right)=3 k^{6}+12 k^{3}-k^{2}+$ $9+\frac{4 k^{2}-7}{\left(k^{3}+k+2\right)^{2}}+\frac{18 k}{\left(k^{3}+k+2\right)^{3}}+\frac{12}{\left(k^{3}+k+2\right)^{4}}>0$ for $x \geq k^{3}+k+2$, we obtain that $g_{2}(x)$ is strictly increasing when $x \geq k^{3}+k+2$. Using $n \geq k^{3}+k+2$, we have $g_{2}(n) \geq g_{2}\left(k^{3}+k+2\right)$. 


$$
\begin{aligned}
f_{1}^{(2)}\left(n-k-1-\frac{1}{n}\right) & =6 n^{2}-12 k n+\frac{18 k}{n}+\frac{12}{n^{2}}+4 k^{2}-16 \\
& >6 n^{2}-12 k n+4 k^{2}-16 \\
& =g_{3}(n)>0,
\end{aligned}
$$

where $g_{3}(x)=6 x^{2}-12 k x+4 k^{2}-16$. For the last inequality, it is easy to see that $g_{3}(x)$ is strictly increasing when $x \geq k^{3}+k+2$. Using $n \geq k^{3}+k+2$, we obtain $g_{3}(n) \geq g_{3}\left(k^{3}+k+2\right)=6 k^{6}+24 k^{3}-2 k^{2}+8>0$.

$$
\begin{aligned}
& f_{1}^{(3)}\left(n-k-1-\frac{1}{n}\right)=6\left(3 n-\frac{4}{n}-3 k\right)>0 . \\
& f_{1}^{(4)}\left(n-k-1-\frac{1}{n}\right)=24>0 .
\end{aligned}
$$

Hence, by the Fourier-Budan Theorem (See, e.g., [57]), there is no root of $f_{1}(x)$ in the interval $\left[n-k-1-\frac{1}{n},+\infty\right)$. Then, by Lemma 1.17, all subgraphs of $G_{n, k}^{1}$ have spectral radius less than $n-k-1-\frac{1}{n}$.

(ii) Since the proofs are very similar, we omit the details.

This completes the proof of Lemma 6.9.

Proof of Theorem 6.3. Note that $\delta(G) \geq \kappa(G) \geq k$. By Lemmas 1.18 and 1.20 , we have

$$
n-k-1-\frac{1}{n}<\rho(G) \leq \frac{k-1}{2}+\sqrt{2 e(G)-n k+\frac{(k+1)^{2}}{4}} .
$$

Therefore, when $n \geq k^{3}+k+2$, we obtain

$$
\begin{aligned}
e(G) & >\frac{1}{2}\left[n^{2}-(2 k+1) n+\frac{3 k+1}{n}+\frac{1}{n^{2}}+2 k^{2}+k-2\right] \\
& >\frac{n^{2}-(2 k+1) n+2 k^{2}+k-2}{2}>\left(\begin{array}{c}
n-k-2 \\
2
\end{array}\right)+(k+2)^{2} .
\end{aligned}
$$

By Theorem 6.8, $G$ is hamiltonian or $\operatorname{cl}(G) \in\left\{G_{n, k}^{1}, G_{n, k}^{2}, G_{n, k}^{3}, G_{n, k}^{4}, G_{n, k}^{5}\right\}$. Since $K_{n-k} \subseteq G_{n, k}^{1}$, by Lemma 1.17, we have $\rho\left(G_{n, k}^{1}\right)>\rho\left(K_{n-k}\right)=n-k-1$. 
Combining the above with Lemma 6.9, we conclude that $G=G_{n, k}^{1}$.

This completes the proof of Theorem 6.3.

We continue this chapter with counterparts of the above results for traceability.

Theorem 6.10. Let $G$ be a $k$-connected graph of order $n \geq 6 k+16$, where $k \geq 1$. If $e(G)>\left(\begin{array}{c}n-k-3 \\ 2\end{array}\right)+(k+2)(k+3)$, then $G$ is traceable unless $c l_{n-1}(G) \in\left\{\underline{G}_{n, k}^{1}, \underline{G}_{n, k}^{2}, \underline{G}_{n, k}^{3}, \underline{G}_{n, k}^{4}, \underline{G}_{n, k}^{5}\right\}$.

Proof. Let $G^{\prime}=G \vee K_{1}$. Then we have $\kappa\left(G^{\prime}\right)=\kappa(G)+1 \geq k+1, n\left(G^{\prime}\right)=$ $n+1=6(k+1)+11$ and $e\left(G^{\prime}\right)=e(G)+n>\left(\begin{array}{c}n-k-3 \\ 2\end{array}\right)+(k+2)(k+3)+n=$ $\left(\begin{array}{c}n-k-2 \\ 2\end{array}\right)+(k+3)^{2}$. By Theorem 6.8, we know $G^{\prime}$ is hamiltonian unless $c l_{n+1}\left(G^{\prime}\right) \in\left\{G_{n+1, k+1}^{1}, G_{n+1, k+1}^{2}, G_{n+1, k+1}^{3}, G_{n+1, k+1}^{4}, G_{n+1, k+1}^{5}\right\}$. Hence, $G$ is traceable unless $c l_{n-1}(G) \in\left\{\underline{G}_{n, k}^{1}, \underline{G}_{n, k}^{2}, \underline{G}_{n, k}^{3}, \underline{G}_{n, k}^{4}, \underline{G}_{n, k}^{5}\right\}$.

This completes the proof of Theorem 6.10.

Lemma 6.11. Let $G$ be a graph of order $n$ with $\delta(G) \geq k$, where $n \geq k^{3}+k^{2}+$ $k+3$ and $k \geq 1$.

(i) If $G$ is a proper subgraph of $\underline{G}_{n, k}^{1}$, then $\rho(G)<n-k-2-\frac{1}{n}$.

(ii) If $G \in\left\{\underline{G}_{n, k}^{2}, \underline{G}_{n, k}^{3}, \underline{G}_{n, k}^{4}, \underline{G}_{n, k}^{5}\right\}$, then $\rho(G)<n-k-2-\frac{1}{n}$.

Proof. (i) For $\underline{G}_{n, k}^{1}=K_{k} \vee\left(K_{n-2 k-1}+(k+1) K_{1}\right)$, let $X$ be the set of vertices with degree $k$, let $Y$ be the set of neighbors of $X$, and let $Z$ be the remaining set of $n-2 k-1$ vertices. Assume that $G$ is a proper subgraph obtained from $\underline{G}_{n, k}^{1}$ by deleting one edge $u v \in E\left(\underline{G}_{n, k}^{1}\right)$. Similarly as in the proof of Lemma 6.9, the case $u, v \in Z$ yields the largest spectral radius.

Let us consider the following partition $\pi$ of $V(G)$ in $X_{1}=X, X_{2}=Y$, $X_{3}=Z \backslash\{u, v\}$, and $X_{4}=\{u, v\}$. It is easy to check that this partition is 
equitable, and that the corresponding adjacency matrix of $G / \pi$ is as follows.

$$
A(G / \pi)=\left(\begin{array}{cccc}
0 & k & 0 & 0 \\
k+1 & k-1 & n-2 k-3 & 2 \\
0 & k & n-2 k-4 & 2 \\
0 & k & n-2 k-3 & 0
\end{array}\right)
$$

By using the Laplace expansion for the calculation of determinants, we obtain the characteristic polynomial of $A(G / \pi)$ :

$$
\begin{aligned}
f_{2}(x)= & x^{4}+(k-n+5) x^{3}+\left(-k^{2}+2 k-3 n+10\right) x^{2}+(k n-2 n-2 k \\
& \left.+k^{2} n-6 k^{2}-2 k^{3}+6\right) x+2 k n-6 k+2 k^{2} n-10 k^{2}-4 k^{3} .
\end{aligned}
$$

We can obtain the following derivatives of $f_{2}(x)$ with respect to $x$, by standard analysis:

$$
\begin{aligned}
f_{2}^{\prime}(x)= & 4 x^{3}+3(k-n+5) x^{2}+2\left(-k^{2}+2 k-3 n+10\right) x+k n-2 n-2 k \\
& +k^{2} n-6 k^{2}-2 k^{3}+6, \\
f_{2}^{(2)}(x)= & 12 x^{2}+6(k-n+5) x+2\left(-k^{2}+2 k-3 n+10\right), \\
f_{2}^{(3)}(x)= & 24 x+6(k-n+5), \\
f_{2}^{(4)}(x)= & 24 .
\end{aligned}
$$

By substitution and simple but tedious calculations, we obtain

$$
\begin{aligned}
f_{2}\left(n-k-2-\frac{1}{n}\right)= & n^{2}-\left(k^{3}+k^{2}+k+3\right) n+\frac{k^{3}+3 k^{2}}{n}+\frac{2 k^{2}+5 k+1}{n^{2}} \\
& +\frac{3 k+3}{n^{3}}+\frac{1}{n^{4}}+k^{4}+k^{3}-2 k^{2}-k+1 \\
> & n^{2}-\left(k^{3}+k^{2}+k+3\right) n+k^{4}+k^{3}-2 k^{2}-k+1 \\
= & h_{1}(n) \geq 0,
\end{aligned}
$$

where $h_{1}(x)=x^{2}-\left(k^{3}+k^{2}+k+3\right) x+k^{4}+k^{3}-2 k^{2}-k+1$. For the last inequality, obviously, the function $h_{1}(x)$ is strictly increasing when $x \geq$ 
$k^{3}+k^{2}+k+3$. Using $n \geq k^{3}+k^{2}+k+3$, we obtain $f_{2}\left(n-k-2-\frac{1}{n}\right)=$ $h_{1}(n) \geq h_{1}\left(k^{3}+k^{2}+k+3\right)=k^{4}+k^{3}-2 k^{2}-k+1 \geq 0$.

$$
\begin{aligned}
f_{2}^{\prime}\left(n-k-2-\frac{1}{n}\right)= & n^{3}-3(k+1) n^{2}+\left(2 k^{2}+5 k\right) n-\frac{4 k^{2}+10 k-1}{n} \\
& -\frac{9(k+1)}{n^{2}}-\frac{4}{n^{3}}-k^{3}-3 k^{2}+6 k+6 \\
= & h_{2}(n) \geq h_{2}\left(k^{3}+k^{2}+k+3\right) \\
= & k^{9}+3 k^{8}+3 k^{7}+7 k^{6}+11 k^{5}+4 k^{4}+7 k^{3}+5 k^{2} \\
& +3 k+6-\frac{4 k^{2}+10 k-1}{k^{3}+k^{2}+k+3}-\frac{9 k+9}{\left(k^{3}+k^{2}+k+3\right)^{2}} \\
& -\frac{4}{\left(k^{3}+k^{2}+k+3\right)^{3}} \\
\geq & k^{9}+3 k^{8}+3 k^{7}+7 k^{6}+11 k^{5}+4 k^{4}+7 k^{3}+5 k^{2} \\
& +3 k+6-\frac{13}{6}-\frac{1}{2}-\frac{1}{54}>0,
\end{aligned}
$$

where $h_{2}(x)=x^{3}-3(k+1) x^{2}+\left(2 k^{2}+5 k\right) x-\frac{4 k^{2}+10 k-1}{x}-\frac{9(k+1)}{x^{2}}-\frac{4}{x^{3}}-$ $k^{3}-3 k^{2}+6 k+6$. For the first inequality, the first derivative of $h_{2}(x)$ is $h_{2}^{\prime}(x)=3 x^{2}-6(k+1) x+(2 k+5) k+\frac{4 k^{2}+10 k-1}{x^{2}}+\frac{18(k+1)}{x^{3}}+\frac{12}{x^{4}}$. Since

$$
\begin{aligned}
h_{2}^{\prime}(x) \geq & h_{2}^{\prime}\left(k^{3}+k^{2}+k+3\right) \\
= & 3 k^{6}+6 k^{5}+3 k^{4}+12 k^{3}+11 k^{2}-k+9 \\
& +\frac{4 k^{2}+10 k-1}{\left(k^{3}+k^{2}+k+3\right)^{2}}+\frac{18 k+18}{\left(k^{3}+k^{2}+k+3\right)^{3}}+\frac{12}{\left(k^{3}+k^{2}+k+3\right)^{4}} \\
> & 0,
\end{aligned}
$$

we have $h_{2}(x)$ is strictly increasing when $x \geq k^{3}+k^{2}+k+3$. Using $n \geq$ $k^{3}+k^{2}+k+3$, we obtain $h_{2}(n) \geq h_{2}\left(k^{3}+k^{2}+k+3\right)$. 


$$
\begin{aligned}
f_{2}^{(2)}\left(n-k-2-\frac{1}{n}\right) & =6 n^{2}-(12 k+12) n+\frac{18 k+18}{n}+\frac{12}{n^{2}}+4 k^{2}+10 k-10 \\
& >6 n^{2}-(12 k+12) n+4 k^{2}+10 k-10 \\
& =h_{3}(n)>0 .
\end{aligned}
$$

For the last inequality, let $h_{3}(x)=6 x^{2}-(12 k+12) x+4 k^{2}+10 k-10$. It is easy to see that $h_{3}(x)$ is strictly increasing when $x \geq k^{3}+k^{2}+k+3$. Using $n \geq k^{3}+k^{2}+k+3$, we obtain $h_{3}(n) \geq h_{3}\left(k^{3}+k^{2}+k+3\right)=6 k^{6}+12 k^{5}+$ $6 k^{4}+24 k^{3}+22 k^{2}-2 k+8>0$.

$$
\begin{aligned}
& f_{2}^{(3)}\left(n-k-2-\frac{1}{n}\right)=18 n-\frac{24}{n}-18 k-18>0 . \\
& f_{2}^{(4)}\left(n-k-2-\frac{1}{n}\right)=24>0 .
\end{aligned}
$$

Hence, by the Fourier-Budan Theorem (See, e.g., [57]), there is no root of $f_{2}(x)$ in the interval $\left[n-k-2-\frac{1}{n},+\infty\right)$. Then by Lemma 1.17, all subgraphs of $\underline{G}_{n, k}^{1}$ have spectral radius less than $n-k-2-\frac{1}{n}$.

(ii) Since the proofs are similar, we omit the details.

This completes the proof of Lemma 6.11.

Proof of Theorem 6.4. Note that $\delta(G) \geq \kappa(G) \geq k$. By Lemmas 1.18 and 1.20, we have

$$
n-k-2-\frac{1}{n}<\rho(G) \leq \frac{k-1}{2}+\sqrt{2 e(G)-n k+\frac{(k+1)^{2}}{4}} .
$$

Therefore, when $n \geq k^{3}+k^{2}+k+3$, we obtain

$$
\begin{aligned}
e(G) & >\frac{1}{2}\left[n^{2}-(2 k+3) n+\frac{3 k+3}{n}+\frac{1}{n^{2}}+2 k^{2}+4 k\right] \\
& >\frac{n^{2}-(2 k+3) n+2 k^{2}+4 k}{2} \\
& >\left(\begin{array}{c}
n-k-3 \\
2
\end{array}\right)+(k+2)(k+3) .
\end{aligned}
$$


By Theorem 6.10, $G$ is traceable or $c l_{n-1}(G) \in\left\{\underline{G}_{n, k}^{1}, \underline{G}_{n, k}^{2}, \underline{G}_{n, k}^{3}, \underline{G}_{n, k}^{4}, \underline{G}_{n, k}^{5}\right\}$. Since $K_{n-k-1} \subseteq \underline{G}_{n, k}^{1}$, by Lemma 1.17, we have $\rho\left(\underline{G}_{n, k}^{1}\right)>\rho\left(K_{n-k-1}\right)=n-$ $k-2$. Combining this with Lemma 6.11, we get $G=\underline{G}_{n, k}^{1}$.

This completes the proof of Theorem 6.4. 


\section{Chapter 7}

\section{Hamilton-connectivity of $k$-connected graphs}

As counterparts to the results of Chapter 6 , in this chapter we continue to study $k$-connected graphs with a focus on Hamilton-connecivity. We will present and prove sufficient conditions for a $k$-connected graph to be Hamiltonconnected in terms of its spectral radius, thereby improving the known analogues for graphs with minimum degree $\delta(G) \geq k$.

\subsection{Introduction}

We start with a result that provides a sufficient condition on the spectral radius for graphs with minimum degree $\delta(G) \geq k$ to be Hamilton-connected.

Theorem 7.1 (Chen, Hou and Qian [10]). Let $G$ be a graph of order $n \geq 6 k^{2}-$ $8 k+5$ with $\delta(G) \geq k \geq 2$. If $\rho(G)>\frac{k-1}{2}+\sqrt{n^{2}-(3 k-1) n+\frac{k^{2}+10 k-15}{4}}$, then $G$ is Hamilton-connected unless $c l_{n+1}(G)=K_{2} \vee\left(K_{n-k-1}+K_{k-1}\right) \operatorname{or~cl}_{n+1}(G)=$ $K_{k} \vee\left(K_{n-2 k+1}+(k-1) K_{1}\right)$.

Here $c l_{n+1}(G)$ is the Bondy-Chvátal closure for Hamilton-connectivity that we introduced earlier. In this chapter, our main results give sufficient conditions for Hamilton-connectivity of $k$-connected graphs in terms of their 
spectral radius, that improve Theorem 7.1. Before stating our main results, we need to introduce some families of graphs.

For $n \geq 2 k$ and $k \geq 2$, we define $H_{n, k}^{1}=K_{k} \vee\left(K_{n-2 k+1}+(k-1) K_{1}\right)$. For the other class, we start with a graph consisting of two vertex-disjoint graphs $(k-1) K_{1}$ and $K_{n-k}$, and an additional new vertex $v$. Let $V\left((k-1) K_{1}\right)=X$, $V\left(K_{n-k}\right)=Y$, and $Y_{2} \subseteq Y$ with $\left|Y_{2}\right|=k-1$. Then by $H_{n, k}^{2}$ we denote the graph obtained from $(k-1) K_{1}+K_{n-k}+\{v\}$ by joining $X$ to $Y_{2}$, and $v$ to $X$, $Y_{2}$, and an arbitrary vertex in $Y \backslash Y_{2}$ (See the graph sketched in Figure 7.1).

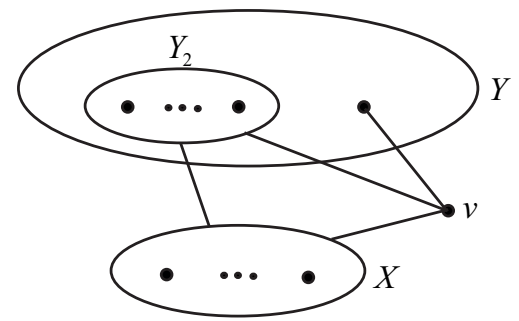

FIGURE 7.1: The graph $H_{n, k}^{2}$.

Similarly, for $n \geq 2 k+1$, let $V\left((k-1) K_{1}\right)=X, V\left(K_{n-k}\right)=Y$, where $X_{1} \subset X$ with $\left|X_{1}\right|=k-2$ and $X_{2}=X \backslash X_{1}$, and $Y_{2} \subseteq Y$ with $\left|Y_{2}\right|=k$. We use $H_{n, k}^{3}$ to denote the graph obtained from $(k-1) K_{1}+K_{n-k}+\{v\}$ by joining $X$ to $Y_{2}$, and $v$ to $X_{2}$ and $Y_{2}$ (See the graph sketched at the left side in Figure 7.2). For the next class, let $V\left(k K_{1}\right)=X, V\left(K_{n-k}\right)=Y$, where $X_{1} \subset X_{2}$ with $\left|X_{1}\right|=k-1$ and $X_{2}=X \backslash X_{1}$, and let $Y_{1}$ and $Y_{2}$ be disjoint subsets of $Y$, with $\left|Y_{1}\right|=k$ and $\left|Y_{2}\right|=1$. Denote by $H_{n, k}^{4}$ the graph obtained from $k K_{1}+K_{n-k}$ by joining $X$ to $Y_{1}$ and $X_{2}$ to $Y_{2}$ (See the right side of Figure 7.2). We also define $H_{n, k}^{5}=K_{k} \vee\left(K_{n-2 k}+k K_{1}\right)$ and $H_{n, k}^{6}=K_{k} \vee\left(K_{n-2 k}+K_{1, k-1}\right)$.

For $n \geq 2 k+2$, we define $H_{n, k}^{7}=K_{k+1} \vee\left(K_{n-2 k-1}+k K_{1}\right)$.

We also need to define five special graphs $G_{i}(1 \leq i \leq 5)$. These graphs are sketched in Figure 7.3, where the ellipses denote a complete subgraph $K_{n-2}$.

Next, we present our main results.

Theorem 7.2. Let $G$ be a $k$-connected graph of order $11 k+11$, where $k \geq 2$. If $\rho(G)>\frac{k-1}{2}+\sqrt{n^{2}-(3 k+3) n+\frac{13 k^{2}+38 k+25}{4}}$, then $G$ is Hamilton-connected 

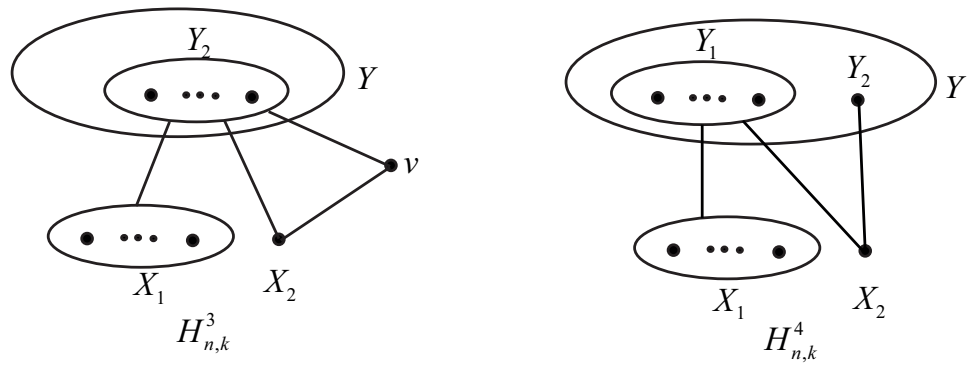

Figure 7.2: The graphs $H_{n, k}^{3}$ and $H_{n, k}^{4}$.
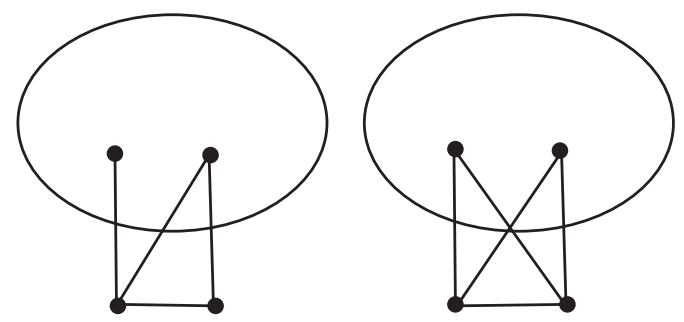

$G_{1}$

$G_{2}$
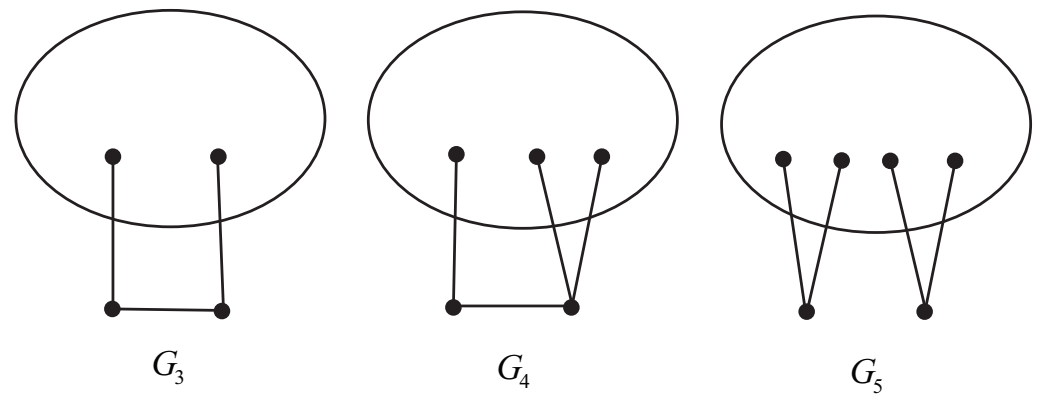

FIGURE 7.3: The graphs $G_{1}-G_{5}$. 
unless $c l_{n+1}(G) \in\left\{H_{n, k}^{1}, H_{n, k}^{3}, H_{n, k}^{4}, H_{n, k}^{5}, H_{n, k}^{7}, H_{4}(k=2,3), G_{i}(1 \leq i \leq 5)\right\}$.

We postpone the prove and the definition of $H_{4}$ to Section 7.3. It is obvious that Theorem 7.2 is an improvement of Theorem 7.1. To further specify the exceptional graphs, we also prove the following theorem.

Theorem 7.3. Let $G$ be a $k$-connected graph of order $n \geq k^{3}-k^{2}+k+2$, where $k \geq 2$. If $\rho(G)>n-k-\frac{1}{n}$, then $G$ is Hamilton-connected unless $G=H_{n, k}^{1}$.

The rest of the paper is organized as follows. In Section 7.2, we will give some useful techniques and necessary lemmas which will be used in our proofs. In Section 7.3, we present one important structural theorem, one useful lemma, as well as the proofs of Theorems 7.2 and 7.3. In Section 7.4, we give some proofs that we postponed in Section 7.3.

\subsection{Preliminaries}

The following is a generalization of the Hamilton-connected counterpart of Dirac's theorem due to Chvátal.

Lemma 7.4 (Chvátal [12]). A graph $G$ with at least three vertices is Hamiltonconnected if $\kappa(G) \geq \alpha(G)+1$.

The definition of the $k$-closure of a graph was given several times in previous chapters. In the proofs of our main results, we need the following result which is due to Bondy and Chvátal [5].

Lemma 7.5 (Bondy and Chvátal [5]). A graph $G$ is Hamilton-connected if and only if $c l_{n+1}(G)$ is hamiltonian.

We end this section with the following lemma that gives upper bounds for the spectral radius of some special graphs.

Lemma 7.6. Let $G$ be a graph of order $n$ with $\delta(G) \geq k$, where $k \geq 2$.

(i) For $n \geq k^{3}-k^{2}+k+2$, if $G$ is a proper subgraph of $H_{n, k}^{1}$, then $\rho(G)<$ $n-k-\frac{1}{n}$. 
(ii) For $n \geq k^{3}-k^{2}+k+2$, if $G \in\left\{H_{n, k}^{3}, H_{n, k}^{4}, H_{n, k}^{5}, H_{n, k}^{7}, H_{4}\right\}$, then $\rho(G)<$ $n-k-\frac{1}{n}$.

(iii) For $k=2$, if $G=G_{i}(1 \leq i \leq 5)$, then $\rho(G)<n-2-\frac{1}{n}$

Proof. (i) For $G=H_{n, k}^{1}$, let $X$ be the set of vertices with degree $k$, let $Y$ be the neighbor set of $X$, and let $Z$ be remaining set of vertices. Suppose $G^{\prime}$ is the subgraph obtained from $G$ by deleting one edge. There are three types for $G^{\prime}$, which are denoted by $G_{1}^{\prime}, G_{2}^{\prime}, G_{3}^{\prime}$ and depicted in Figure 7.4. We have $G_{2}^{\prime}=G_{1}^{\prime}-v z+u z$ and $G_{3}^{\prime}=G_{2}^{\prime}-v z+u z$, which are Kelmans transformations. Then, by Lemma 1.14, we know that $\rho\left(G_{1}^{\prime}\right) \leq \rho\left(G_{2}^{\prime}\right) \leq \rho\left(G_{3}^{\prime}\right)$. So it is sufficient to prove $\rho\left(G_{3}^{\prime}\right)<n-k-\frac{1}{n}$.
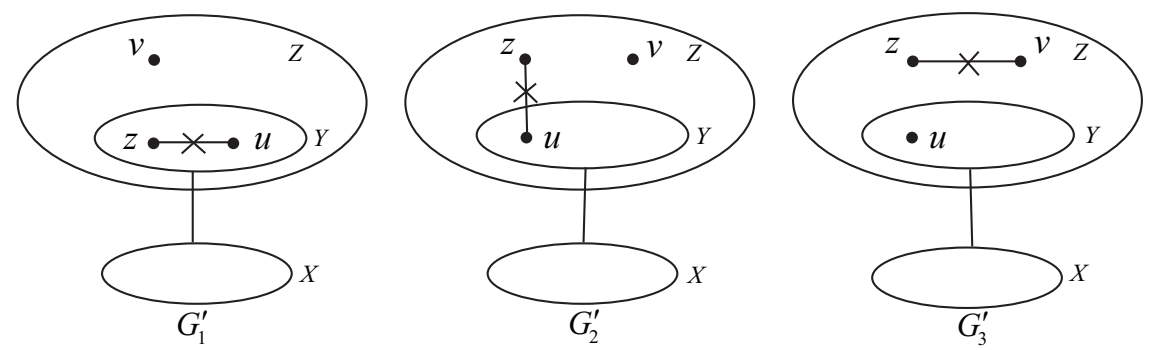

Figure 7.4: The graphs $G_{1}^{\prime}, G_{2}^{\prime}$ and $G_{3}^{\prime}$.

Consider the following partition, denoted by $\pi$, of $V\left(G_{3}^{\prime}\right)$ in $X_{1}=X$, $X_{2}=Y, X_{3}=Z \backslash\{v, z\}$ and $X_{4}=\{v, z\}$. This partition is equitable and the adjacency matrix of the quotient matrix of $G_{3}^{\prime}$ is as follows.

$$
A\left(G_{3}^{\prime} / \pi\right)=\left(\begin{array}{cccc}
0 & k & 0 & 0 \\
k-1 & k-1 & n-2 k-1 & 2 \\
0 & k & n-2 k-2 & 2 \\
0 & k & n-2 k-1 & 0
\end{array}\right) .
$$

The characteristic polynomial of $A\left(G_{3}^{\prime} / \pi\right)$ is equal to:

$$
\begin{aligned}
f(x)= & x^{4}+(k-n+3) x^{3}-\left(k^{2}-4 k+3 n-4\right) x^{2}+\left(4 k-2 n-k n+k^{2} n\right. \\
& \left.-2 k^{3}+2\right) x+2 k-2 k n+2 k^{2} n+2 k^{2}-4 k^{3} .
\end{aligned}
$$


By simple calculations, we obtain

$$
\begin{aligned}
f^{\prime}(x)= & 4 x^{3}+3(k-n+3) x^{2}-2\left(k^{2}-4 k+3 n-4\right) x+4 k-2 n-k n \\
& +k^{2} n-2 k^{3}+2 \\
f^{(2)}(x)= & 12 x^{2}+6(k-n+3) x-2\left(k^{2}-4 k+3 n-4\right) ; \\
f^{(3)}(x)= & 24 x+6(k-n+3) \\
f^{(4)}(x)= & 24 .
\end{aligned}
$$

By using the software package Mathematica, we can get

$$
\begin{aligned}
f\left(n-k-\frac{1}{n}\right)= & n^{2}-\left(k^{3}-k^{2}+k+1\right) n+k^{4}-3 k^{3}+5 k-3 \\
& +\frac{k^{3}-k^{2}-2 k+4}{n}+\frac{2 k^{2}-5 k+1}{n^{2}}+\frac{3 k-3}{n^{3}}+\frac{1}{n^{4}} \\
> & n^{2}-\left(k^{3}-k^{2}+k+1\right) n+k^{4}-3 k^{3}+5 k-3-\frac{1}{4} \\
= & g_{1}(n) \geq g_{1}\left(k^{3}-k^{2}+k+2\right) \\
= & k^{4}-2 k^{3}-k^{2}+6 k-\frac{5}{4}>0,
\end{aligned}
$$

where $g_{1}(x)=x^{2}-\left(k^{3}-k^{2}+k+1\right) x+k^{4}-3 k^{3}+5 k-3-\frac{1}{4}$. It is obvious that $g_{1}(x)$ is increasing when $x \geq k^{3}-k^{2}+k+2$. Since $n \geq k^{3}-k^{2}+k+2$, the inequality (7.1) holds.

$$
\begin{aligned}
f^{\prime}\left(n-k-\frac{1}{n}\right)= & n^{3}-(3 k-3) n^{2}+\left(2 k^{2}-5 k\right) n-\frac{4 k^{2}-10 k-1}{n} \\
& -\frac{9 k-9}{n^{2}}-\frac{4}{n^{3}}-k^{3}+k^{2}+8 k-10 \\
= & g_{2}(n) \geq g_{2}\left(k^{3}-k^{2}+k+2\right) \\
= & k^{9}-3 k^{8}+3 k^{7}+8 k^{6}-19 k^{5}+11 k^{4}+22 k^{3}-27 k^{2}+10 k
\end{aligned}
$$




$$
\begin{aligned}
& +10-\frac{4 k^{2}-10 k-1}{k^{3}-k^{2}+k+2}-\frac{9 k-9}{\left(k^{3}-k^{2}+k+2\right)^{2}} \\
& -\frac{4}{\left(k^{3}-k^{2}+k+2\right)^{3}} \\
> & k^{9}-3 k^{8}+3 k^{7}+8 k^{6}-19 k^{5}+11 k^{4}+22 k^{3}-27 k^{2}+10 k \\
& +10-1-1-\frac{4}{513} \\
> & 0,
\end{aligned}
$$

where $g_{2}(x)=x^{3}-(3 k-3) x^{2}+\left(2 k^{2}-5 k\right) x-\frac{4 k^{2}-10 k-1}{x}-\frac{9 k-9}{x^{2}}-\frac{4}{x^{3}}-k^{3}+$ $k^{2}+8 k-10$. For inequality (7.2), since $g_{2}^{\prime}(x)=3 x^{2}-2(3 k-3) x+2 k^{2}-$ $5 k+\frac{4 k^{2}-10 k-1}{x^{2}}+\frac{18 k-18}{x^{3}}+\frac{12}{x^{4}}$ and

$$
\begin{aligned}
g_{2}^{\prime}\left(k^{3}-k^{2}+k+2\right)= & 3 k^{6}-6 k^{5}+3 k^{4}+18 k^{3}-19 k^{2}+k+24 \\
& +\frac{4 k^{2}-10 k-1}{\left(k^{3}-k^{2}+k+2\right)^{2}}+\frac{18 k-18}{\left(k^{3}-k^{2}+k+2\right)^{3}} \\
& +\frac{12}{\left(k^{3}-k^{2}+k+2\right)^{4}} \\
> & 3 k^{6}-6 k^{5}+3 k^{4}+18 k^{3}-19 k^{2}+k+24-\frac{5}{64} \\
> & 0,
\end{aligned}
$$

we obtain that $g_{2}(x)$ is an increasing function when $x \geq k^{3}-k^{2}+k+2$. Because $n \geq k^{3}-k^{2}+k+2$, the inequality (7.2) holds.

$$
\begin{aligned}
f^{(2)}\left(n-k-\frac{1}{n}\right) & =6 n^{2}-(12 k-12) n+\frac{18 k-18}{n}+\frac{12}{n^{2}}+4 k^{2}-10 k-10 \\
& >6 n^{2}-(12 k-12) n+4 k^{2}-10 k-10 \\
& =g_{3}(n) \geq g_{3}\left(k^{3}-k^{2}+k+2\right) \\
& =6 k^{6}-12 k^{5}+6 k^{4}+36 k^{3}-38 k^{2}+2 k+38 \\
& >0
\end{aligned}
$$

where $g_{3}(x)=6 x^{2}-(12 k-12) x+4 k^{2}-10 k-10$. 


$$
\begin{aligned}
& f^{(3)}\left(n-k-\frac{1}{n}\right)=18 n-\frac{24}{n}-18 k+18>0 \\
& f^{(4)}\left(n-k-\frac{1}{n}\right)=24>0 .
\end{aligned}
$$

Hence, by the Fourier-Budan Theorem (See, e.g., [57]), there is no root of $f(x)$ in the interval $\left[n-k-\frac{1}{n},+\infty\right)$. Then by Lemma 1.17, all subgraphs of $H_{n, k}^{1}$ have spectral radius less than $n-k-\frac{1}{n}$.

(ii) For $G=H_{4}$ (we give the definition in Section 7.3), $H_{4} \subseteq K_{2 k-1} \vee$ $\left(K_{n-3 k+1}+k K_{1}\right)$. Similarly as before, we can prove that $\rho\left(K_{2 k-1} \vee\left(K_{n-3 k+1}+\right.\right.$ $\left.\left.k K_{1}\right)\right)<n-k-\frac{1}{n}$. Then by Lemma 1.17 , we have $\rho\left(H_{4}\right)<n-k-\frac{1}{n}$.

For the other graphs in (ii) and (iii), the proofs are very similar, hence we omit the details.

This completes the proof of Lemma 7.6.

\subsection{The proofs of our results}

We begin this section with a lemma about four families of Hamilton-connected graphs. Firstly we need to define these four types of special graphs, in a similar way as we introduced the exceptional graphs in the previous section. We also refer to Figure 7.5 to clarify the graphs. As before, let $V\left((k-1) K_{1}\right)=X$ and $V\left(K_{n-k}\right)=Y$. Suppose $Y_{2} \subseteq Y$ and $\left|Y_{2}\right|=k-2$. Then $H_{1}$ (sketched in the upper part of Figure 7.5) is the graph obtained from $(k-1) K_{1}+K_{n-k}+\{v\}$ by joining $Y_{2}$ to $X$ and $v$, and joining each of $a(a \geq 1)$ vertices of $X$ to two (private) vertices in $Y \backslash Y_{2}$ (meaning that the neighbors of these $a$ vertices do not overlap), and each of $b(b \geq 1)$ vertices in $X$ with $v$ and one (private) vertex in $Y \backslash Y_{2}$, where $a+b=k-1$. Then denote by $Y_{1}$ the neighbor set of $X$ in $Y \backslash Y_{2}$. Set $X=X_{1} \cup X_{2}$, where $\left|X_{1}\right|=a \geq 1$ and $\left|X_{2}\right|=b \geq 1$, and $Y_{2} \subseteq Y$ with $\left|Y_{2}\right|=k-1$. The graph $H_{2}$ is obtained from $(k-1) K_{1}+K_{n-k}+\{v\}$ by joining $Y_{2}$ to $X$ and $v$, and $v$ to $X_{2}$, and then joining each vertex of $X_{1}$ to one (private) vertex in $Y \backslash Y_{2}$, and denoting by $Y_{1}$ the neighbor set of $X_{1}$ in $Y \backslash Y_{2}$ (See the lower part of Figure 7.5). 

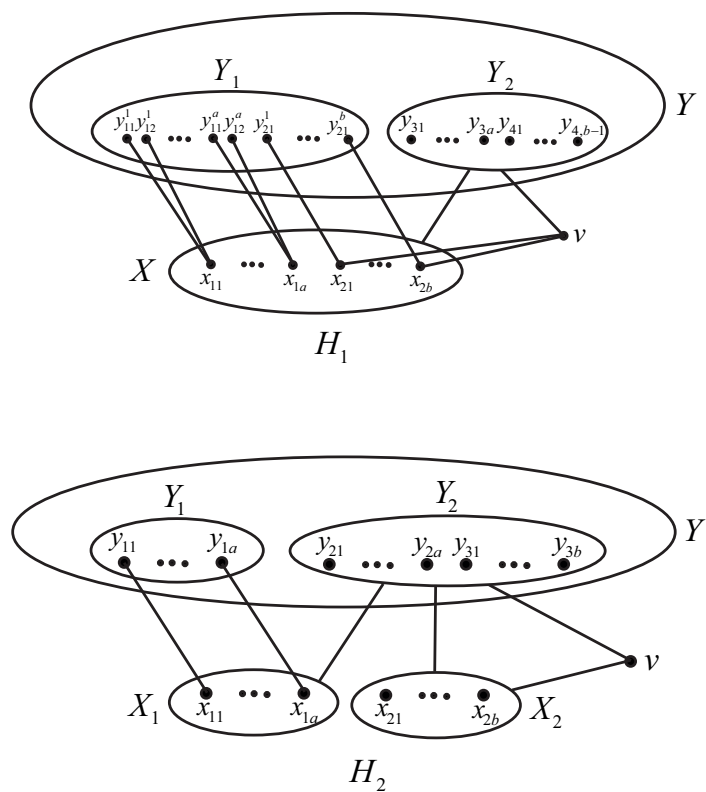

FIGURE 7.5: The graphs $H_{1}$ and $H_{2}$

For the next pair of graph families, we refer to Figure 7.6 for further clarification. Here, let $V\left(k K_{1}\right)=X$ and $V\left(K_{n-k}\right)=Y$. Suppose $X=X_{1} \cup X_{2}$ and $Y_{1}, Y_{2} \subseteq Y$, where $\left|X_{1}\right|=k-2,\left|X_{2}\right|=2,\left|Y_{1}\right|=k$ and $\left|Y_{2}\right|=2$. Now, $H_{3}$ is the graph obtained from $k K_{1}+K_{n-k}$ by joining $Y_{1}$ to $X$, then joining each vertex of $X_{2}$ to one (private) vertex of $Y_{2}$. Suppose $Y_{11}, Y_{12} \subseteq Y$, where $\left|Y_{11}\right|=k$ and $\left|Y_{12}\right|=k-1$. Now, $H_{4}$ is the graph obtained from $k K_{1}+K_{n-k}$ by joining $Y_{12}$ to $X$, and then joining each vertex of $X$ to one (private) vertex of $Y_{11}$ (See the lower part of Figure 7.6).

We first state the following lemma.

Lemma 7.7. Let $H_{i}$ be defined as above $(i=1,2,3,4)$. Then

(i) $\mathrm{H}_{1}, \mathrm{H}_{2}$, and $\mathrm{H}_{3}$ are all Hamilton-connected.

(ii) $\mathrm{H}_{4}(k \geq 4)$ is Hamilton-connected.

Proof. Since the proofs for all graphs in (i) are similar, in (i) below we only give the details for $H_{1}$ here, and postpone the other details to the appendix (the concluding section of this chapter). 

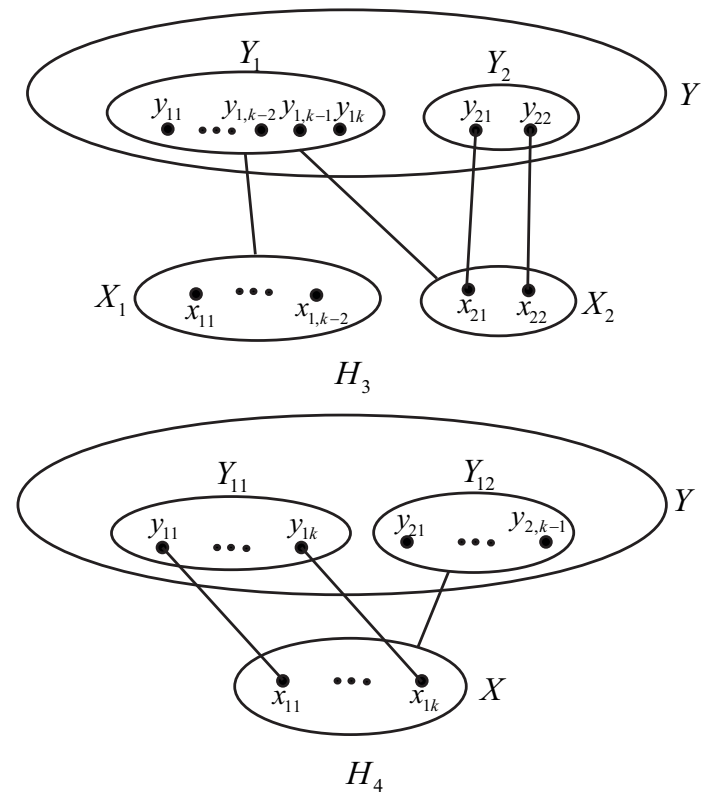

FIgURE 7.6: The graphs $H_{3}$ and $H_{4}$.

(i) We first recall some notations. For two distinct vertices $u$ and $v$ in a graph $G$, we use $u P v$ to denote a Hamilton path in $G$ connecting $u$ and $v$. Let $P_{u v}$ and $P_{w z}$ be two disjoint paths. Then, we denote by $P_{u v} \bigsqcup P_{w z}$ a path obtained from $P_{u v}$ and $P_{w z}$ by joining $v$ and $w$ with an edge.

We start by labeling the vertices of the earlier defined sets $X$ and $Y_{i}$ $(i=1,2)$ of $H_{1}$ (referring to Figure 7.5) as $x_{11}, \ldots, x_{1 a}, x_{21}, \ldots, x_{2 b}$; $y_{11}^{1}, y_{12}^{1}, \ldots, y_{11}^{a}, y_{12}^{a}, y_{21}^{1}, \ldots, y_{21}^{b} ; y_{31}, \ldots, y_{3 a}, y_{41}, \ldots, y_{4, b-1}$, where $a \geq 1$, $b \geq 1$ and $a+b=k-1$. Since $H[Y]$ is a clique, there always exists a Hamilton path between any two vertices in the remaining subgraph of $H[Y]$ from which possibly some vertices are deleted. This is indicated by $P^{\prime}$ at the right hand side of the below equations. Let $R_{i}=y_{11}^{i} x_{1 i} y_{12}^{i}, Q_{1}=x_{11} y_{31} \cdots x_{1 a} y_{3 a}$ and $Q_{2}=x_{21} y_{41} \cdots x_{2, b-1} y_{4, b-1}$. The following are nine types of Hamilton paths in $H_{1}$ :

$$
y_{11}^{1} P y_{12}^{1}=y_{11}^{1} Q_{1} Q_{2} v x_{2 b} y_{21}^{b} P^{\prime} y_{12}^{1}
$$




$$
\begin{aligned}
y_{11}^{1} P x_{11} & =y_{11}^{1}\left(\bigsqcup_{i=2}^{a} R_{i}\right) y_{21}^{1} Q_{2} v x_{2 b} y_{21}^{b} P^{\prime} y_{12}^{1} x_{11} ; \\
y_{11}^{1} P y_{31} & =\left(\bigsqcup_{i=1}^{a} R_{i}\right) y_{21}^{1} Q_{2} v x_{2 b} y_{21}^{b} P^{\prime} y_{31} ; \\
y_{11}^{1} P v & =\left(\bigsqcup_{i=1}^{a} R_{i}\right) y_{21}^{1} Q_{2} x_{2 b} y_{21}^{b} P^{\prime} y_{31} v ; \\
x_{11} P x_{21} & =x_{11} y_{11}^{1}\left(\bigsqcup_{i=2}^{a} R_{i}\right) y_{21}^{2}\left(Q_{2}-y_{21} y_{41}\right) x_{2 b} v y_{31} P^{\prime} y_{21}^{1} x_{21} ; \\
x_{11} P v & =x_{11} y_{11}^{1}\left(\bigsqcup_{i=2}^{a} R_{i}\right) y_{21}^{1} Q_{2} x_{2 b} y_{21}^{b} P^{\prime} y_{31} v ; \\
x_{11} P y_{31} & =x_{11} y_{11}^{1}\left(\bigsqcup_{i=2}^{a} R_{i}\right) y_{21}^{1} Q_{2} v x_{2 b} y_{21}^{b} P^{\prime} y_{31} ; \\
y_{31} P y_{32} & =y_{31} x_{11} y_{11}^{1}\left(\bigsqcup_{i=2}^{a} R_{i}\right) y_{21}^{1} Q_{2} v x_{2 b} y_{21}^{b} P^{\prime} y_{32} ; \\
y_{31} P v & =y_{31} x_{11} y_{11}^{1}\left(\bigsqcup_{i=2}^{a} R_{i}\right) y_{21}^{1} Q_{2} P^{\prime} y_{21}^{b} x_{2 b} v
\end{aligned}
$$

This covers all possible cases, so we conclude that $H_{1}$ is Hamilton-connected.

(ii) Referring to Figure 7.6, we label the vertices of $X, Y_{11}, Y_{12}$ of $H_{4}$ as $x_{11}, \ldots, x_{1 k} ; y_{11}, \ldots, y_{1 k} ; y_{21}, \ldots, y_{2, k-1}$. Let $R_{i}=y_{2 i} x_{1,2 i-1} y_{1,2 i-1} y_{1,2 i} x_{1,2 i}$ and $Q=x_{11} y_{21} \cdots x_{1, k-1} y_{2, k-1}$. The following are seven types of Hamilton paths in $\mathrm{H}_{4}$ :

$$
\begin{aligned}
y_{11} P y_{12} & =y_{11} Q x_{1 k} y_{1 k} P^{\prime} y_{12} ; \\
y_{11} P y_{2, k-1} & =y_{11}\left(Q-x_{1, k-1} y_{2, k-1}\right) x_{1, k-1} y_{1, k-1} P^{\prime} y_{1 k} x_{1 k} y_{2, k-1} ; \\
y_{11} P x_{1 k} & =y_{11} Q P^{\prime} y_{1 k} x_{1 k} ; \\
x_{11} P x_{1 k} & =x_{11} y_{11} P^{\prime} y_{12}\left(Q-y_{21} x_{11}\right) y_{1 k} x_{1 k} ; \\
x_{11} P y_{2, k-1} & =\left(Q-x_{1, k-1} y_{2, k-1}\right) x_{1, k-1} y_{1, k-1} P^{\prime} y_{1 k} x_{1 k} y_{2, k-1} ;
\end{aligned}
$$




$$
\begin{aligned}
& \left.y_{21} P y_{2, k-1}=\left(\bigsqcup_{i=1}^{k / 2} R_{i}\right) y_{2, \frac{k+2}{2}} P^{\prime} y_{2, k-1} \text { (when } k \text { is even and } k \geq 4\right) ; \\
& \left.y_{21} P y_{2, k-1}=\left(\bigsqcup_{i=1}^{(k-1) / 2} R_{i}\right) y_{2, \frac{k+1}{2}} P^{\prime} y_{1 k} x_{1 k} y_{2, k-1} \text { (when } k \text { is odd and } k \geq 5\right) .
\end{aligned}
$$

This covers all cases, hence when $k \geq 4, H_{4}$ is Hamilton-connected.

Next, we state and prove one of the key results of this chapter.

Theorem 7.8. Let $G$ be a $k$-connected graph of order $n \geq 11 k+11$ with $k \geq 2$. If $e(G)>\left(\begin{array}{c}n-k-1 \\ 2\end{array}\right)+(k+1)(k+2)$, then $G$ is Hamilton-connected unless $c l_{n+1}(G) \in\left\{H_{n, k}^{1}, H_{n, k}^{3}, H_{n, k}^{4}, H_{n, k}^{5}, H_{n, k}^{7}, H_{4}(k=2,3), G_{i}(1 \leq i \leq 5)\right\}$.

Proof. Let $H=c l_{n+1}(G)$. If $H$ is Hamilton-connected, then by Lemma 7.5, so is $G$. Now we suppose $H$ is not Hamilton-connected. Noting that $H$ is $k$-connected, using Lemma 7.4, we have $\alpha(H)>k-1$. Since $e(H) \geq e(G)>$ $\left(\begin{array}{c}n-k-1 \\ 2\end{array}\right)+(k+1)(k+2)$, as in the proof of Theorem 4.4 in Chapter 4, we get $\omega(H) \geq n-k$. We claim that $\omega(H) \leq n-k+1$. In fact, if $\omega(H) \geq n-k+2$, then $\alpha(H) \leq k-1$, a contradiction. Hence, we divide the proof into two cases.

Case 1. $\omega(H)=n-k+1$.

In this case, we have $\alpha(H)=k$. Set $V(H)=X \cup Y$, where $H[X]=(k-$ 1) $K_{1}, H[Y]=K_{n-k+1}$, and $X$ together with a vertex $w \in Y$ is a maximum independent set. Let $Y_{1}=N_{H[Y]}(X)$. Then $d_{H}(y) \geq n-k+1$ for $y \in Y_{1}$, and $X$ is adjacent to $Y_{1}$. Since $d_{H}(w)=n-k$, we have $d_{H}(x)=k$ for each $x \in X$. Hence $\left|Y_{1}\right|=k$ and we obtain that $H=H_{n, k}^{1}=K_{k} \vee\left(K_{n-2 k+1}+(k-1) K_{1}\right)$.

Case 2. $\omega(H)=n-k$.

In this case, we have $\alpha(H)=k$ or $k+1$. We complete the proof by considering these two subcases separately.

Subcase 2.1. $\alpha(H)=k$.

The first situation is that $V(H)=X \cup Y$, where $H[X]=k K_{1}, H[Y]=K_{n-k}$, and $X$ is a maximum independent set. So every vertex in $Y$ must be adjacent to some $x$ in $X$; otherwise $\alpha(H)=k+1$. Set $Y=Y_{1} \cup Y_{2}$, where $y \in Y_{1}$ has 
only one neighbor in $X$, and $y \in Y_{2}$ has at least two neighbors in $X$. Hence $d_{H}(y)=n-k$ for $y \in Y_{1}$, and $d_{H}(y) \geq n-k+1$ for $y \in Y_{2}$. Then $X$ is adjacent to $Y_{2}$. Let $X_{1}=N_{H[X]} Y_{1}$ and $X_{2}=X \backslash X_{1}$. If $Y_{1}=\emptyset$, then $H=k K_{1} \vee K_{n-k}$, which is Hamilton-connected, a contradiction. If $Y_{2}=\emptyset$, then obviously $H$ is Hamilton-connected, a contradiction. Hence, $Y_{1} \neq \emptyset$ and $Y_{2} \neq \emptyset$. If $\left|X_{1}\right|=1$, then $x \in X_{1}$ is adjacent to $Y$, which contradicts that $\omega(H)=n-k$. If $\left|X_{1}\right| \geq 2$, then $\left|Y_{2}\right| \leq k-1$; otherwise $d_{H}(x) \geq k+1$ for $x \in X_{1}$ and $Y_{1}$ will be adjacent to $X_{1}$. Now, the vertices in $X_{2}$ have degree less than $k$, a contradiction.

The second situation is that $V(H)=X \cup Y \cup\{v\}$, where $H[X]=(k-1) K_{1}$, $H[Y]=K_{n-k}, v \notin X \cup Y$, and $X$ together with a vertex $w \in Y$ is a maximum independent set. We use $X_{1}, X_{2}, Y_{1}, Y_{2}$ to denote the same sets as in the first situation. Similarly, $X$ is adjacent to $Y_{2}$, and $v$ is adjacent to $Y_{2}$ and has at least one neighbor in $X$. If $v$ is adjacent to $Y \backslash\left(Y_{1} \cup Y_{2}\right)$, then all possible $w$ have degree $n-k$. Hence, $d_{H}(x)=k$ for every $x \in X$. We have $Y_{1} \neq \emptyset$; otherwise $v$ is adjacent to $Y$, which contradicts that $\omega(H)=n-k$. So $X_{1} \neq \emptyset$. If $X_{2}=\emptyset$, then $\left|Y_{2}\right| \leq k-1$. When $\left|Y_{2}\right|=k-1$, every vertex in $X_{1}$ has only one neighbor in $Y_{1}$, which results in $v$ having no neighbor in $X$, a contradiction. So $\left|Y_{2}\right| \leq k-2$. Let $\left|Y_{2}\right|=t$. Then $x \in N_{H[X]}(v)$ has $k-t-1$ neighbors in $Y_{1}$, and $x \in X \backslash N_{H[X]}(v)$ has $k-t$ neighbors in $Y_{1}$. When $t \leq k-3$, since every vertex in $X$ has at least two neighbors in $Y_{2}$, it is easy to check that $H$ is Hamilton-connected. When $t=k-2$, we have $H_{1} \subseteq H$, and by Lemma 7.7 (i), $H$ is Hamilton-connected, a contradiction. If $X_{2} \neq \emptyset$, then we claim $\left|Y_{2}\right|=k-1$. Indeed, if $\left|Y_{2}\right| \leq k-2$, then $x \in X_{2}$ has degree at most $k-1$, a contradiction. If $\left|Y_{2}\right| \geq k$, then $x \in X_{1}$ has degree at least $k+1$, a contradiction. Therefore, every vertex in $X_{1}$ has a one-to-one neighbor in $Y_{1}$, and $v$ is adjacent to $X_{2}$. Then $H_{2} \subseteq H$, and by Lemma 7.7 (i), we get that $H$ is Hamilton-connected, a contradiction.

Next, we discuss the case that there exists a vertex $w$ with degree $n-k-1$. Then $d_{H}(x)=k$ or $k+1$ for $x \in X$.

If $Y_{1}=\emptyset$, then $X_{1}=\emptyset$, and $X_{2}$ is adjacent to $Y_{2}$. If $d_{H}(x)=k+1$ for all $x \in X$, then $\left|Y_{2}\right|=k$ and $v$ is adjacent to $X$. When $v$ has no neighbors in $Y \backslash Y_{2}$, we have $H=H_{n, k}^{6}$. It is easy to check that $H_{n, k}^{6}$ is Hamilton-connected when $k \geq 3$. We can get a contradiction except for $k=2$. In this case, $H=H_{n, 2}^{6}=G_{2}$. When $v$ has at least one neighbor in $Y \backslash Y_{2}$, we can easily see 
that $H$ is Hamilton-connected, a contradiction. If $d_{H}(x)=k$ for all $x \in X$, then $\left|Y_{2}\right|=k-1$ and $v$ is adjacent to $X$. Also, $v$ must have at least one neighbor in $Y \backslash Y_{2}$; otherwise $Y_{2}$ is a cut set. If $v$ has only one neighbor in $Y \backslash Y_{2}$, then $d_{H}(v)+d_{H}(w)=n+k-2$. When $k \geq 3, v$ and $w$ are adjacent, a contradiction. When $k=2, H=H_{n, k}^{2}(k=2)=G_{1}$. If $v$ has more than one neighbor in $Y \backslash Y_{2}$, then $d_{H}(v) \geq 2 k$ and $d_{H}(v)+d_{H}(w) \geq 2 k+n-k-1 \geq$ $n+1$, which means $v$ is adjacent to all vertices in $Y \backslash Y_{2}$, a contradiction. If $d_{H}(x)=k$ for some vertices in $X$, and $d_{H}(x)=k+1$ for the other vertices in $X$, then $\left|Y_{2}\right|=k$ and the vertices that have degree $k+1$ are adjacent to $v$. If $v$ has at least two neighbors in $X$ or has a neighbor in $Y \backslash Y_{2}$, then it is easy to check that $H$ is Hamilton-connected, a contradiction. If $v$ has only one neighbor in $X$ and has no neighbors in $Y \backslash Y_{2}$, then $H=H_{n, k}^{3}$.

If $Y_{2}=\emptyset$, then $X_{2}=\emptyset$. When $k \geq 3$, then it is obvious that $H$ is Hamiltonconnected, a contradiction. When $k=2$, we can see that there is only one vertex $x_{1}$ in $X$, and $x_{1}$ must be adjacent to $v$. If $d_{H}\left(x_{1}\right)=k+1=3$, then there are two neighbors of $x_{1}$ in $Y_{1}$, say $y_{1}$ and $y_{2}$. In this case, if $v$ is adjacent to at least one vertex in $Y \backslash Y_{1}$, then $H$ is Hamilton-connected, a contradiction. If $v$ is only adjacent to $y_{1}$ or $y_{2}$, then $H=G_{1}$. If $v$ has no neighbors in $Y \backslash Y_{1}$ and is adjacent to $y_{1}$ and $y_{2}$, then $H=G_{2}$. If $d_{H}\left(x_{1}\right)=k=2$, then there is one neighbor of $x_{1}$ in $Y_{1}$, say $y_{1}$. We have that $v$ has neighbors in $Y \backslash Y_{1}$; otherwise $\left\{y_{1}\right\}$ will be a cut vertex. In this case, if $v$ is adjacent to $y_{1}$, then there is only one neighbor of $v$ in $Y \backslash Y_{1}$ and $H=G_{1}$. If $v$ is not adjacent to $y_{1}$, then there are at most two neighbors of $v$ in $Y \backslash Y_{1}$ and $H=G_{3}$ or $G_{4}$.

If $Y_{1} \neq \emptyset$ and $Y_{2} \neq \emptyset$, when $X_{2}=\emptyset$, then $d_{H}(x)=k$ for $x \in X_{1}$ and $\left|Y_{2}\right| \leq k-2$. If $\left|Y_{2}\right| \leq k-3$, then every vertex in $X$ has at least two neighbors in $Y_{1}$, and it is easy to check that $H$ is Hamilton-connected, a contradiction. If $\left|Y_{2}\right|=k-2$, set $X_{1}=X_{11} \cup X_{12}$, and $v$ is adjacent to $X_{12}$. Then every vertex in $X_{11}$ has two neighbors in $Y_{1}$, and every vertex in $X_{12}$ has only one neighbor in $Y_{1}$. It is easy to see that $H_{1} \subseteq H$, and by Lemma 7.7 (i), we get a contradiction. When $X_{2} \neq \emptyset$, we have $d_{H}(x)=k$ for $x \in X_{2}$ since $d_{H}(y)=n-k$ for $y \in Y_{1}$. Hence $\left|Y_{2}\right|=k-1$ and $v$ is adjacent to $X_{2}$. In this case, we have $H_{2} \subseteq H$, and by Lemma 7.7 (i), $H$ is Hamilton-connected, a contradiction. 
Subcase 2.2. $\alpha(H)=k+1$.

Set $V(H)=X \cup Y$, where $H[X]=k K_{1}, H[Y]=K_{n-k}$, and $X$ together with one vertex $w \in Y$ is a maximum independent set. Since $d_{H}(w)=n-k-1$, we have $d_{H}(x)=k$ or $k+1$ for $x \in X$. Let $X_{1}=\left\{x \mid d_{H}(x)=k, x \in X\right\}$, $X_{2}=\left\{x \mid d_{H}(x)=k+1, x \in X\right\}, Y_{1}=N_{H[Y]}\left(X_{1}\right)$, and $Y_{2}=N_{H[Y]}\left(X_{2}\right) \backslash Y_{1}$.

If $X_{1}=\emptyset$, then $X$ is adjacent to $Y_{2}$ and $\left|Y_{2}\right|=k+1$. Hence $H=H_{n, k}^{7}=$ $K_{k+1} \vee\left(K_{n-2 k-1}+k K_{1}\right)$.

If $X_{1} \neq \emptyset$ and $X_{2} \neq \emptyset$, then $d_{H}(y) \geq n-k+1$ for $y \in Y_{1}$, since $y$ has neighbors both in $X_{1}$ and $X_{2}$. So every vertex in $X_{1}$ is adjacent to every vertex in $Y_{1}$, and $\left|Y_{1}\right|=k$. Then every vertex in $X_{2}$ has a one-to-one neighbor in $Y_{2}$, and $\left|X_{2}\right|=\left|Y_{2}\right|$. When $\left|X_{2}\right|=\left|Y_{2}\right|=1, H=H_{n, k}^{4}$. When $\left|X_{2}\right|=\left|Y_{2}\right| \geq 2$, then $H_{3} \subseteq H$, where $H_{3}$ is the graph when $\left|X_{2}\right|=\left|Y_{2}\right|=2$. By Lemma 7.7 (i), $H$ is Hamilton-connected, a contradiction.

If $X_{2}=\emptyset$, then $Y_{2}=\emptyset$. Let $Y_{11} \subseteq Y_{1}$ be the set of vertices with only one neighbor in $X$, and $Y_{12} \subseteq Y_{1}$ be the set of vertices with at least two neighbors in $X$. Then $Y_{12}$ is adjacent to $X_{1}$. If $Y_{11}=\emptyset$, then $\left|Y_{12}\right|=k$ and $H=H_{n, k}^{5}=K_{k} \vee\left(K_{n-2 k}+k K_{1}\right)$. If $Y_{12}=\emptyset$, then obviously $H$ is Hamiltonconnected when $k \geq 3$, a contradiction. When $k=2, G=G_{5}$. If $Y_{11} \neq \emptyset$ and $Y_{12} \neq \emptyset$, then $\left|Y_{12}\right| \leq k-1$. When $\left|Y_{12}\right| \leq k-2$, then every vertex in $X_{1}$ has at least two neighbors in $Y_{11}$, and it is easy to check that $H$ is Hamiltonconnected, a contradiction. When $\left|Y_{12}\right|=k-1$, then every vertex in $X_{1}$ has a one-to-one neighbor in $Y_{11}$. In this case, we have $H=H_{4}$, and by Lemma 7.7 (ii), we get a contradiction, except for $k=2,3$.

This completes the proof of Theorem 7.8

Proof of Theorem 7.2. Combining Lemmas 1.18 and 1.20, we have

$$
\begin{gathered}
\frac{k-1}{2}+\sqrt{n^{2}-(3 k+3) n+\frac{13 k^{2}+38 k+25}{4}}<\rho(G) \\
\leq \frac{k-1}{2}+\sqrt{2 e(G)-n k+\frac{(k+1)^{2}}{4}}
\end{gathered}
$$

By simple straightforward calculations, we obtain that $e(G)>\left(\begin{array}{c}n-k-1 \\ 2\end{array}\right)+$ $(k+1)(k+2)$. Then, using Theorem 7.8, we get that $G$ is Hamilton-connected 
unless $c l_{n+1}(G) \in\left\{H_{n, k}^{1}, H_{n, k}^{3}, H_{n, k}^{4}, H_{n, k}^{5}, H_{n, k}^{7}, H_{4}(k=2,3), G_{i}(1 \leq i \leq 5)\right\}$.

Proof of Theorem 7.3. Suppose that $G$ is not Hamilton-connected. Combining this with Lemmas 1.18 and 1.20 , we have

$$
n-k-\frac{1}{n}<\rho(G) \leq \frac{k-1}{2}+\sqrt{2 e(G)-n k+\frac{(k+1)^{2}}{4} .}
$$

Hence

$$
\begin{aligned}
e(G) & >\frac{1}{2}\left[n^{2}-(2 k-1) n+\frac{3 k-1}{n}+\frac{1}{n^{2}}+2 k^{2}-2 k-2\right] \\
& >\frac{1}{2}\left[n^{2}-(2 k+3) n+3 k^{2}+9 k+6\right] \\
& =\left(\begin{array}{c}
n-k-1 \\
2
\end{array}\right)+(k+1)(k+2) .
\end{aligned}
$$

By Theorem 7.8, we know $c l_{n+1}(G) \in\left\{H_{n, k}^{1}, H_{n, k}^{3}, H_{n, k}^{4}, H_{n, k}^{5}, H_{n, k}^{7}, H_{4}(k=\right.$ 2,3)\}. Since $K_{n-k+1} \subseteq H_{n, k}^{1}$, using Lemma 1.17, we have $\rho\left(H_{n, k}^{1}\right)>\rho\left(K_{n-k+1}\right)=$ $n-k$. Furthermore, for $c l_{n+1}(G) \varsubsetneqq H_{n, k}^{1}$ and $G \subseteq\left\{H_{n, k}^{3}, H_{n, k}^{4}, H_{n, k}^{5}, H_{n, k}^{7}, H_{4}(k=\right.$ 2,3), $\left.G_{i}(1 \leq i \leq 5)\right\}$, using Lemmas 1.17 and 7.6, we can get a contradiction.

\subsection{Appendix}

Proof of Lemma 7.7 (i). For $\mathrm{H}_{2}$, similarly as in the given proofs for some cases of Lemma 7.7, we label the vertices of $X_{i}, Y_{i}(i=1,2)$ of $H_{2}$ as $x_{11}, \ldots, x_{1 a}$;

$x_{21}, \ldots, x_{2 b} ; y_{11}, \ldots, y_{1 a} ; y_{21}, \ldots, y_{2 a} ; y_{31}, \ldots, y_{3 b}$ (referring to Figure 7.5 ), where $a \geq 1, b \geq 1$ and $a+b=k-1$. Since $H[Y]$ is a clique, there always is a Hamilton path between any two vertices in the remaining subgraph of $H[Y]$ where possibly some vertices are deleted. As before, this is indicated by the $P^{\prime}$ in the right hand side of the below equations. When $a$ is even and $a \geq 4$, let $R_{1 i}=y_{2 i} x_{1,2 i} y_{1,2 i} y_{1,2 i+1} x_{1,2 i+1}, R_{2 i}=y_{2 i} x_{1,2 i-1} y_{1,2 i-1} y_{1,2 i} x_{1,2 i}$, 
$Q_{1}=x_{21} y_{31} \cdots x_{2 b} y_{3 b}$ and $Q_{2}=y_{31} x_{21} \cdots y_{3 b} x_{2 b}$. The following are fourteen types of Hamilton paths in $\mathrm{H}_{2}$ :

$$
\begin{aligned}
& y_{11} P y_{1 a}=y_{11} x_{11}\left(\bigsqcup_{i=1}^{(a-2) / 2} R_{1 i}\right) y_{2, \frac{a}{2}} x_{1 a} y_{2, \frac{a+2}{2}} v Q_{1} P^{\prime} y_{1 a} ; \\
& y_{11} P y_{3 b}=y_{11} x_{11}\left(\bigsqcup_{i=1}^{(a-2) / 2} R_{1 i}\right) y_{2, \frac{a}{2}} x_{1 a} y_{1 a} P^{\prime}\left(Q_{2}-y_{3 b} x_{2 b}\right) v x_{2 b} y_{3 b} ; \\
& y_{11} P x_{11}=y_{11} Q_{2} v\left(\bigsqcup_{i=1}^{(a-2) / 2} R_{1 i}\right) y_{2, \frac{a}{2}} x_{1 a} y_{1 a} P^{\prime} y_{2, \frac{a+2}{2}} x_{11} ; \\
& y_{11} P x_{21}=y_{11} x_{11}\left(\bigsqcup_{i=1}^{(a-2) / 2} R_{1 i}\right) y_{2, \frac{a}{2}} x_{1 a} y_{1 a} P^{\prime} y_{2, \frac{a+2}{2}} x_{22}\left(Q_{1}-x_{21} y_{31}\right) v x_{21} ; \\
& y_{11} P v=y_{11} x_{11}\left(\bigsqcup_{i=1}^{(a-2) / 2} R_{1 i}\right) y_{2, \frac{a}{2}} x_{1 a} y_{1 a} P^{\prime} Q_{2} v ; \\
& x_{11} P x_{1 a}=x_{11}\left(\bigsqcup_{i=1}^{(a-2) / 2} R_{1 i}\right) y_{2, \frac{a}{2}} v Q_{1} P^{\prime} y_{1 a} x_{1 a} ; \\
& x_{11} P x_{2 b}=x_{11}\left(\bigsqcup_{i=1}^{(a-2) / 2} R_{1 i}\right) y_{2, \frac{a}{2}} x_{1 a} y_{1 a} P^{\prime}\left(Q_{2}-y_{3 b} x_{2 b}\right) y_{3 b} v x_{2 b} ; \\
& x_{21} P v=x_{21}\left(\bigsqcup_{i=1}^{a / 2} R_{2 i}\right) y_{2, \frac{a+2}{2}} P^{\prime} y_{31}\left(Q_{1}-x_{21} y_{31}\right) v ; \\
& x_{11} P y_{3 b}=x_{11}\left(\bigsqcup_{i=1}^{(a-2) / 2} R_{1 i}\right) y_{2, \frac{a}{2}} x_{1 a} y_{1 a} P^{\prime}\left(Q_{2}-y_{3 b} x_{2 b}\right) v x_{2 b} y_{3 b} ; \\
& x_{11} P v=x_{11}\left(\bigsqcup_{i=1}^{a-2) / 2} x_{1 i}\right) y_{2, \frac{a}{2}} x_{1 a} y_{1 a} P^{\prime} Q_{2} v ; \\
& x_{3 b}=y_{21} x_{11} y_{11} P^{\prime} y_{12} x_{12}\left(\bigsqcup_{i=2}^{a / 2} R_{2 i}\right) y_{2, \frac{a+2}{2}} x_{21} v\left(Q_{1}-x_{21} y_{31}\right) ; \\
&\left.x_{2 i}\right) y_{2, \frac{a+2}{2}} P^{\prime} y_{31} v\left(Q_{2}-x_{21} y_{31}\right) ;
\end{aligned}
$$




$$
\begin{gathered}
y_{21} P v=\left(\bigsqcup_{i=1}^{a / 2} R_{2 i}\right) y_{2, \frac{a+2}{2}} P^{\prime} Q_{2} v \\
y_{21} P x_{2 b}=y_{21} x_{11} y_{11} P^{\prime} y_{12} x_{12}\left(\bigsqcup_{i=2}^{a / 2} R_{2 i}\right) y_{2, \frac{a+2}{2}}\left(Q_{2}-y_{3 b} x_{2 b}\right) y_{3 b} v x_{2 b} .
\end{gathered}
$$

This covers all the possible cases, hence $H_{2}$ is Hamilton-connected. When $a=2$, the proof is simpler and therefore omitted. When $a$ is odd, the proof is similar, and also omitted.

For $H_{3}$, as before, we label the vertices of $X_{i}$ and $Y_{i}(i=1,2)$ of $H_{3}$ as $x_{11}, \ldots, x_{1, k-2} ; x_{21}, x_{22} ; y_{11}, \ldots, y_{1 k} ; y_{21}, y_{22}$ (referring to Figure 7.6). Let $Q_{1}=y_{11} x_{11} \cdots y_{1, k-2} x_{1, k-2}$ and $Q_{2}=x_{11} y_{11} \cdots x_{1, k-2} y_{1, k-2}$. The following are ten types of Hamilton paths in $\mathrm{H}_{3}$ :

$$
\begin{aligned}
y_{11} P y_{1 k} & =y_{11} x_{21} y_{21} P^{\prime} y_{22} x_{22}\left(Q_{1}-y_{11} x_{11}\right) y_{1, k-1} x_{11} y_{1 k} ; \\
y_{11} P y_{22} & =Q_{1} y_{1, k-1} x_{21} y_{21} P^{\prime} y_{1 k} x_{22} y_{22} ; \\
y_{11} P x_{11} & =y_{11}\left(Q_{2}-x_{11} y_{11}\right) x_{21} y_{21} P^{\prime} y_{1, k-1} x_{22} y_{22} y_{1 k} x_{11} ; \\
y_{11} P x_{22} & =Q_{1} y_{1, k-1} x_{21} y_{21} P^{\prime} y_{22} x_{22} ; \\
x_{11} P x_{1, k-2} & =\left(Q_{2}-x_{1, k-2} y_{1, k-2}\right) y_{1, k-2} y_{21} x_{21} y_{1, k-1} x_{22} y_{22} P^{\prime} y_{1 k} x_{1, k-2} ; \\
x_{11} P y_{22} & =Q_{2} y_{21} x_{21} y_{1, k-1} P^{\prime} y_{1 k} x_{22} y_{22} ; \\
x_{11} P x_{22} & =Q_{2} x_{21} y_{1, k-1} P^{\prime} y_{22} x_{22} ; \\
y_{21} P y_{22} & =y_{21} x_{21} Q_{1} y_{1, k-1} x_{22} y_{1 k} P^{\prime} y_{22} ; \\
y_{21} P x_{22} & =y_{21} x_{21} Q_{1} y_{1, k-1} P^{\prime} y_{22} x_{22} ; \\
x_{21} P x_{22} & =x_{21} Q_{1} y_{1, k-1} P^{\prime} y_{22} x_{22} .
\end{aligned}
$$

This covers all possible cases, hence $H_{3}$ is Hamilton-connected. 


\section{Chapter 8}

\section{Hamiltoncity of graphs without a large bipartite hole}

In this final chapter, we deal with a recently introduced concept due to McDiarmid and Yolov [49]. An $(s, t)$-bipartite-hole in a graph $G$ consists of two disjoint sets of vertices $S$ and $T$ with $|S|=s$ and $|T|=t$ such that $E(S, T)=\emptyset$. The bipartite-hole-number $\widetilde{\alpha}(G)$ is the smallest integer $r$ that may be written as $r=s+t-1$ for some positive integers $s$ and $t$ such that $G$ does not contain an $(s, t)$-bipartite-hole. In this chapter, we present tight conditions for hamiltonian properties of graphs with a large minimum degree, involving $\widetilde{\alpha}(G)$. One of our main results is that a graph $G$ with at least three vertices is Hamilton-connected if $\delta(G) \geq \widetilde{\alpha}(G)+1$, which improves the analogue of Dirac's theorem for Hamilton-connected graphs.

\subsection{Introduction}

As we mentioned in Chapter 1, many existing sufficient conditions that guarantee a graph to have a Hamilton cycle are based on the traditional degrees of its vertices, and date back to the work of Dirac [16] from the 1950s. Since then, many extensions and generalizations of these results have appeared. Up to now, degree conditions are still an important and well-studied type of 
sufficient conditions for the existence of Hamilton cycles in graphs, as well as for similar hamiltonian properties.

The disjoint union of two complete graphs of the same order shows that Dirac's theorem is sharp. In this example, there clearly is a big bipartite hole (two disjoint sets of vertices with no edges between them). Inspired by this observation, McDiarmid and Yolov [49] presented a tight extremal threshold for the existence of Hamilton cycles in graphs with a large minimum degree and without a large bipartite hole, as follows.

Theorem 8.1 (McDiarmid and Yolov [49]). A graph $G$ with at least three vertices is hamiltonian if $\delta(G) \geq \widetilde{\alpha}(G)$.

The definition of $\widetilde{\alpha}(G)$ has been given as Definition 1.1 in the introductory chapter. Given a positive integer $k$ and a graph $G$, judging whether $\widetilde{\alpha}(G) \geq k$ is an NP-complete decision problem [49]. The above theorem is sharp, in the sense that there exist nonhamiltonian graphs $G$ with $\delta(G)=r=\widetilde{\alpha}(G)-1$ for any positive integer $r$. One example is the complete bipartite graph $G=$ $K_{r, r+1}$, satisfying $\delta(G)=r$ and $\widetilde{\alpha}(G)=r+1$. Furthermore, Theorem 8.1 generalizes Theorem 1.1. Indeed, a graph $G$ has no $(1,\lfloor n / 2\rfloor)$-bipartite-hole if $\delta(G) \geq n / 2$, and hence such graphs satisfy $\widetilde{\alpha}(G) \leq n / 2 \leq \delta(G)$.

In [49], the authors also used Theorem 8.1 to provide a sufficient condition for the existence of many edge-disjoint Hamilton cycles.

Theorem 8.2 (McDiarmid and Yolov [49]). Let $r \geq 0$ be an integer and let $G$ be a graph on at least three vertices such that $\delta(G) \geq(r+1) \widetilde{\alpha}(G)+3 r$. Then $G$ contains $r+1$ edge-disjoint Hamilton cycles.

In this chapter, we continue to study sufficient conditions for hamiltonian properties of graphs with a large minimum degree. The following two sections contain our main results, including sufficient conditions for a graph to be traceable or Hamilton-connected, and the proofs of our results, respectively. 


\subsection{Main results}

We start with the following result that gives a sufficient condition for traceable graphs, as an analogue to the above result and the counterpart of Dirac's theorem for traceable graphs. The proof is not difficult, but we postpone all our proofs to the next section.

Theorem 8.3. A graph $G$ of order $n \geq 3$ is traceable if $\delta(G) \geq \widetilde{\alpha}(G)-1$.

There are examples showing that the result is sharp, similar to the above. Consider, e.g., the nontraceable graph $G=K_{r, r+2}$, for which clearly $\delta(G)=r$ and $\widetilde{\alpha}(G)=r+2$. Furthermore, Theorem 8.3 can be extended to obtain a sufficient condition for the existence of many edge-disjoint Hamilton paths.

Theorem 8.4. Let $r \geq 0$ be an integer, and let $G$ be a graph on at least three vertices such that $\delta(G) \geq(r+1) \widetilde{\alpha}(G)+3 r-1$. Then $G$ contains $r+1$ edgedisjoint Hamilton paths.

Next, we give an analogue of Theorem 8.3 for Hamilton-connected graphs.

Theorem 8.5. A graph $G$ on at least three vertices is Hamilton-connected if $\delta(G) \geq \widetilde{\alpha}(G)+1$.

This result is also sharp since there is a non-Hamilton-connected graph $G$ with $\delta(G)=\widetilde{\alpha}(G)=r$ for any positive integer $r$. An obvious example is the graph $G=K_{r, r}$, satisfying $\delta(G)=\widetilde{\alpha}(G)=r$. An analogue of Dirac's theorem for Hamilton-connected graphs states that a graph $G$ of order $n$ is Hamilton-connected if $\delta(G) \geq \frac{n+1}{2}$. It is not difficult to show that Theorem 8.5 improves this result. For a graph satisfying $\delta(G) \geq \frac{n+1}{2}$, it is easy to check that there is no $\left(1,\left\lfloor\frac{n-1}{2}\right\rfloor\right)$-bipartite-hole. Then $\delta(G) \geq \frac{n+1}{2} \geq \widetilde{\alpha}(G)+1$.

The following generalization of Dirac's theorem for Hamilton-connected graphs is due to Chvátal and Erdős.

Theorem 8.6 (Chvátal and Erdős [11]). A graph $G$ with at least three vertices is Hamilton-connected if $\kappa(G) \geq \alpha(G)+1$.

We observe that the condition of Theorem 8.5 is very similar to that of Theorem 8.6. But comparing these two theorems, neither condition implies 


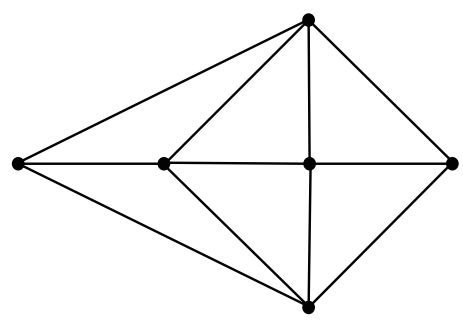

FIgURE 8.1: Graph G.

the other. We first show an example of a graph $G$ meeting the condition of Theorem 8.5 but not of Theorem 8.6. Let $G$ be the graph on vertex set $V(G)=V(A) \cup V(B) \cup V(C)$, where $A=K_{\ell}, B=\overline{K_{k}}, C=K_{k}, \ell \leq k$, and all these subgraphs are mutually vertex-disjoint. Let the edge set of $G$ be defined as $E(G)=E(A) \cup E(C) \cup\{a b \mid a \in V(A), b \in V(B)\} \cup\{b c \mid b \in V(B), c \in V(C)\}$. Obviously, we have $\kappa(G)=k=\alpha(G)$, and $\delta(G)=\ell-1+k \geq \min \{2 \ell+$ $1, k+1\}+1=\widetilde{\alpha}(G)+1$ for $\ell \geq 3$ and $k \geq \ell+3$. In the other direction, the graph $G$ that is depicted in Figure 8.1 satisfies $\kappa(G)=3, \alpha(G)=2$ but $\delta(G)=\widetilde{\alpha}(G)=3$.

In the next section, we will present the details of our proofs of the above theorems.

\subsection{The proofs}

Our proof of Theorem 8.3 is an easy consequence of Theorem 8.1 and the following observation.

Lemma 8.7. (Exercise 18.1.6 on Page 474 of [6]). Let $G$ be a graph. Then $G$ is traceable if and only if $G \vee K_{1}$ is hamiltonian.

Proof of Theorem 8.3. Suppose $H=G \vee K_{1}$ with vertex set $V(H)=V(G) \cup\{v\}$ and edge set $E(H)=E(G) \cup\{v x \mid x \in V(G)\}$. By the definition of the bipartite-hole-number, we know that $\widetilde{\alpha}(H)=\widetilde{\alpha}(G)$. Then $\delta(H)=\delta(G)+1 \geq$ $\widetilde{\alpha}(G)-1+1=\widetilde{\alpha}(G)=\widetilde{\alpha}(H)$. Using Theorem 8.1, we obtain that $H$ is hamiltonian. Then by Lemma $8.7, G$ is traceable.

Next, we present our proof of Theorem 8.4. 
Proof of Theorem 8.4. By induction, it is easy to show that there exist edgedisjoint Hamilton paths $Q_{1}, Q_{2}, \ldots$ one by one, by adapting and applying the condition. The base of the induction is obvious. Suppose we have already shown that there exist such paths $Q_{1}, \ldots, Q_{k}, 1 \leq k \leq r$, and let $P=Q_{1} \cup \cdots \cup Q_{k}$. To show that the next edge-disjoint Hamilton path exists, by Theorem 8.3, it is sufficient to prove that $\delta(G-P) \geq \widetilde{\alpha}(G-P)-1$.

Let $s, t$ be two integers such that $\widetilde{\alpha}(G)+1=s+t$, where $1 \leq s \leq t$, and $G$ has no $(s, t)$-bipartite-hole. We claim that $G-P$ has no $(s, 2 k s+t)$-bipartitehole. In fact, if $|V(G)|<s+2 k s+t$, then obviously the claim holds. If $|V(G)| \geq s+2 k s+t$, then let $U, W \subseteq V(G)$ be two disjoint sets of cardinality $s$ and $2 k s+t$, respectively. Then, $\left|W \backslash N_{P}(U)\right| \geq|W|-\sum_{i=1}^{k}\left|N_{Q_{i}}(U)\right| \geq$ $2 k s+t-2 k s=t$. Since $G$ has no $(s, t)$-bipartite-hole, $G-P$ has no $(s, 2 k s+t)$ bipartite-hole either.

Then, using that $s \leq \frac{\widetilde{\alpha}(G)+1}{2}$, we have $\widetilde{\alpha}(G-P)+1 \leq s+2 k s+t \leq$ $(k+1)(\widetilde{\alpha}(G)+1)$. Hence, we get $\widetilde{\alpha}(G-P)-1 \leq(k+1)(\widetilde{\alpha}(G)+1)-2 \leq$ $(r+1) \widetilde{\alpha}(G)+r-1 \leq \delta(G)-2 r \leq \delta(G-P)$. Now, by Theorem 8.3, there exists a Hamilton path $Q_{k+1}$ which is edge-disjoint from $Q_{1}, \ldots, Q_{k}$.

At the end of this chapter, we present our proof of Theorem 8.5.

Proof of Theorem 8.5. If $\widetilde{\alpha}(G)=1$, then $G$ is complete, and so $G$ is Hamiltonconnected. Hence we may suppose that $\widetilde{\alpha}(G) \geq 2$ and $G$ is not Hamiltonconnected. Then there exist two vertices $u$ and $v$ such that there is no Hamilton path connecting them. By Theorem 8.1, we know $G$ is hamiltonian. Let $C$ be a Hamilton cycle in $G$, and let $|V(C)|=n$. Label the vertices in $V(C)$ with $[n]:=\{1,2, \ldots, n\}$ in order according to the clockwise direction, where $u=n$ and $v=k$ for some $k \notin\{1, n-1, n\}$. For a set $S \subseteq V(C)$, denote by $S^{+}$ the set of successors $x^{+}$on $C$ of elements $x$ in $S$, and denote by $S^{-}$the set of predecessors $x^{-}$. Furthermore, we define $n^{+}=1$ and $1^{-}=n$.

Let $1 \leq s \leq t$ be such that $\widetilde{\alpha}(G)+1=s+t$ and $G$ has no $(s, t)$-bipartitehole. Since $\widetilde{\alpha}(G) \geq 2$, we have $1 \leq s \leq \frac{\widetilde{\alpha}(G)+1}{2}<\widetilde{\alpha}(G)$, and hence

$$
\mid N(1) \cap\{1,2\})|=1 \leq s \leq \delta(G)-2 \leq| N(1) \cap(2, n) \mid=d(1)-2 .
$$


Therefore we can choose $\ell \in(1, n)$ such that $|N(1) \cap(1, \ell]|=s$. We know that 1 is not adjacent to $k+1$ since there is no Hamilton path from $n$ to $k$. Hence, we have $\ell \in(1, k]$ or $\ell \in(k+1, n)$. For $\ell \in(1, k]$, we consider the two cases that $\ell=k$ or $\ell \in(1, k)$. The case that $\ell \in(k+1, n)$ is similar, so we omit it.

Case 1. $\ell=k$.

We describe four situations (referring to Figure 8.2) in which there is a Hamilton path connecting $n$ and $k$, denoted as an $(n, k)-\mathrm{H}$-path in the remainder of the proof.

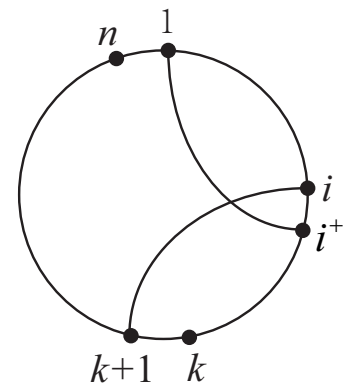

(a)

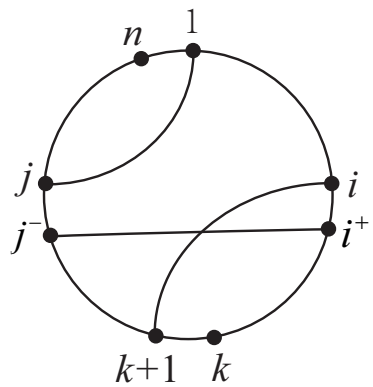

(c)

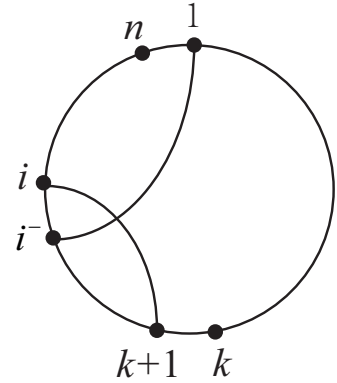

(b)

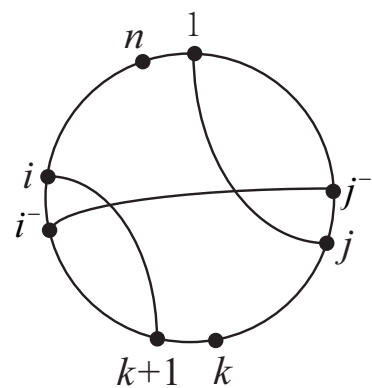

(d)

FigURE 8.2: Situations $(a)-(d)$.

(a) If for some $i \in(1, k]$ we have $i \in N(k+1)$ and $i^{+} \in N(1)$, then $\overleftarrow{C}[n, k+$ 1] $\overleftarrow{C}[i, 1] \vec{C}\left[i^{+}, k\right]$ is an $(n, k)$-H-path.

(b) If for some $i \in(k+1, n]$ we have $i \in N(k+1)$ and $i^{-} \in N(1)$, then $\overleftarrow{C}[n, i] \vec{C}\left[k+1, i^{-}\right] \vec{C}[1, k]$ is an $(n, k)-$ H-path. 
(c) If for some $i \in(1, k)$ and $j \in(k+1, n]$ we have $i \in N(k+1), j \in N(1)$ and $i^{+} j^{-} \in E(G)$, then $\overleftarrow{C}[n, j] \vec{C}[1, i] \vec{C}\left[k+1, j^{-}\right] \vec{C}\left[i^{+}, k\right]$ is an $(n, k)-$ H-path.

(d) If for some $i \in(k+1, n]$ and $j \in(1, k]$ we have $i \in N(k+1), j \in N(1)$ and $i^{-} j^{-} \in E(G)$, then $\overleftarrow{C}[n, i] \vec{C}\left[k+1, i^{-}\right] \overleftarrow{C}\left[j^{-}, 1\right] \vec{C}[j, k]$ is an $(n, k)-$ H-path.

We shall show that at least one of these situations must hold. Suppose for a contradiction that this is not the case. Then for every $k \in(1, n)$

$$
E\left[(N(k+1) \cap(1, k))^{+},\{1\} \cup(N(1) \cap(k+1, n])^{-}\right]=\emptyset,
$$

since (a) and (c) do not hold; and

$$
E\left[(N(k+1) \cap(k+1, n])^{-},(N(1) \cap(1, k])^{-}\right]=\emptyset,
$$

since $(b)$ and $(d)$ do not hold.

Then equation (8.2) implies that $|N(k+1) \cap(k+1, n]|<t$. Since $\mid N(k+$ $1) \cap(1, k)|+| N(k+1) \cap(k+1, n]|+|\{k\} \mid \geq \delta(G)$, we obtain $|N(k+1) \cap(1, k)|>$ $\delta(G)-t-1 \geq \widetilde{\alpha}(G)+1-t-1=s-1$. Hence $|N(k+1) \cap(1, k)| \geq s$. Then equation (8.1) implies that $\left|\{1\} \cup(N(1) \cap(k+1, n])^{-}\right|<t$. Therefore,

$$
\delta(G) \leq|N(1) \cap(1, k]|+|N(1) \cap(k+1, n]|<s+t-1=\widetilde{\alpha}(G) \leq \delta(G)-1,
$$

a contradiction.

Case 2. $\ell \in(1, k)$.

Here, we describe five situations (referring to Figure 8.3) in which there is an $(n, k)-H-p a t h$.

(e) If for some $i \in(1, \ell]$ we have $i \in N(1)$ and $i^{-} \in N(k+1)$, then $\overleftarrow{C}[n, k+$ 1] $\overleftarrow{C}\left[i^{-}, 1\right] \vec{C}[i, k]$ is an $(n, k)-H-p a t h$

(f) If for some $i \in(1, \ell]$ and $j \in(\ell, k]$ we have $i \in N(1), j \in N(k+1)$ and $i^{-} j^{+} \in E(G)$, then $\overleftarrow{C}[n, k+1] \overleftarrow{C}[j, i] \vec{C}\left[1, i^{-}\right] \vec{C}\left[j^{+}, k\right]$ is an $(n, k)-$ H-path. 
( $g$ ) If for some $i \in(1, \ell]$ and $j \in(k+1, n]$ we have $i \in N(1), j \in N(k+1)$ and $i^{-} j^{-} \in E(G)$, then $\overleftarrow{C}[n, j] \vec{C}\left[k+1, j^{-}\right] \overleftarrow{C}\left[i^{-}, 1\right] \vec{C}[i, k]$ is an $(n, k)$ H-path.

(h) If for some $i \in(\ell, k]$ and $j \in(1, \ell]$ we have $i \in N(1), j \in N(k+1)$ and $i^{-} j^{-} \in E(G)$, then $\overleftarrow{C}[n, k+1] \vec{C}\left[j, i^{-}\right] \overleftarrow{C}\left[j^{-}, 1\right] \vec{C}[i, k]$ is an $(n, k)$ H-path.

( $t$ ) If for some $i \in(k+1, n]$ and $j \in(1, \ell]$ we have $i \in N(1), j \in N(k+1)$ and $i^{-} j^{-} \in E(G)$, then $\overleftarrow{C}[n, i] \vec{C}\left[1, j^{-}\right] \overleftarrow{C}\left[i^{-}, k+1\right] \vec{C}[j, k]$ is an $(n, k)$ H-path.

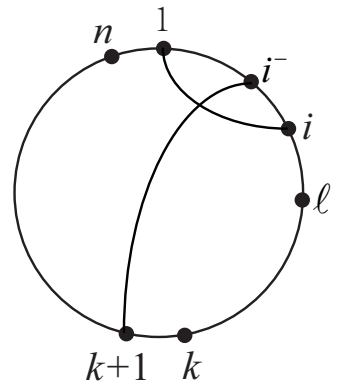

(e)

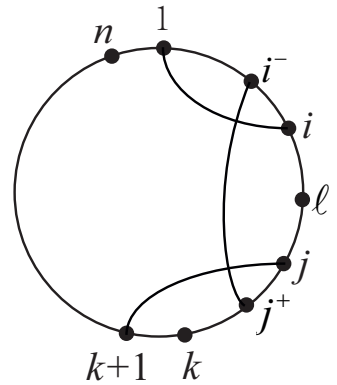

$(f)$

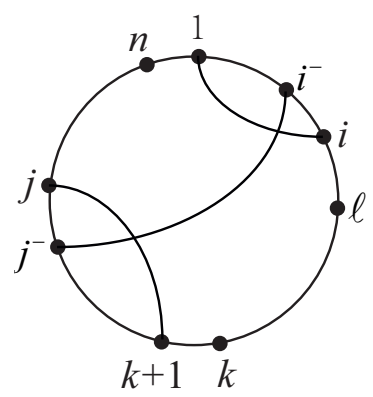

$(g)$

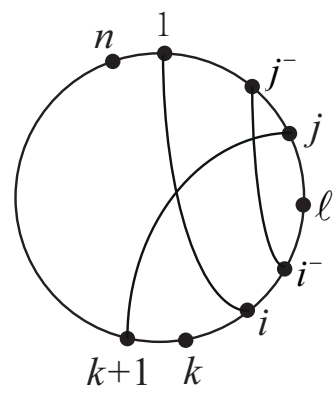

(h)

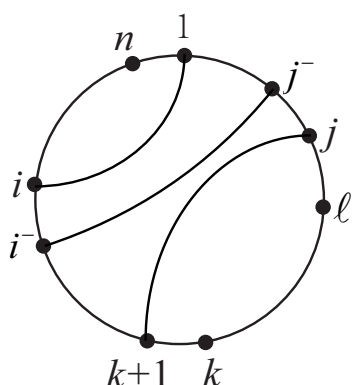

$(t)$

FiguRE 8.3: Situations $(e)-(t)$. 
We shall show that at least one of these situations must hold. Suppose for a contradiction that this is not the case. Then for every $\ell \in(1, k)$

$$
E\left[(N(1) \cap(1, \ell])^{-},(N(k+1) \cap(\ell, k])^{+} \cup(N(k+1) \cap(k+1, n])^{-}\right]=\emptyset,
$$

since $(e),(f)$ and $(g)$ do not hold; and

$$
E\left[(N(1) \cap(\ell, k])^{-} \cup(N(1) \cap(k+1, n])^{-},(N(k+1) \cap(1, \ell])^{-}\right]=\emptyset,
$$

since $(h)$ and $(t)$ do not hold.

Then equation (8.3) implies $\mid(N(k+1) \cap(\ell, k])^{+} \cup(N(k+1) \cap(k+$ $1, n])^{-} \mid<t$. Then

$$
\begin{aligned}
d(k+1)= & |N(k+1) \cap(1, \ell]| \\
& +|N(k+1) \cap(\ell, k]|+|N(k+1) \cap(k+1, n]| \\
= & |N(k+1) \cap(1, \ell]| \\
& +\left|(N(k+1) \cap(\ell, k])^{+}\right|+\left|(N(k+1) \cap(k+1, n])^{-}\right| \\
= & |N(k+1) \cap(1, \ell]| \\
& +\left|(N(k+1) \cap(\ell, k])^{+} \cup(N(k+1) \cap(k+1, n])^{-}\right| \\
& +\left|(N(k+1) \cap(\ell, k])^{+} \cap(N(k+1) \cap(k+1, n])^{-}\right| \\
= & |N(k+1) \cap[1, \ell]| \\
& +\left|(N(k+1) \cap(\ell, k])^{+} \cup(N(k+1) \cap(k+1, n])^{-}\right|+1 \\
\geq & \delta(G) .
\end{aligned}
$$

Now we have $|N(k+1) \cap[1, \ell]|>\delta(G)-t-1 \geq \widetilde{\alpha}(G)+1-t-1=s-1$. Hence $|N(k+1) \cap[1, \ell]| \geq s$. Then equation (8.4) implies $\mid(N(1) \cap(\ell, k])^{-} \cup$ 
$(N(1) \cap(k+1, n])^{-} \mid<t$. Therefore

$$
\begin{aligned}
\delta(G) \leq d(1)= & |N(1) \cap(1, \ell]|+|N(1) \cap(\ell, k]|+|N(1) \cap(k+1, n]| \\
= & |N(1) \cap[1, \ell]|+\left|(N(1) \cap(\ell, k])^{-}\right|+\left|(N(1) \cap(k+1, n])^{-}\right| \\
= & |N(1) \cap[1, \ell]|+\left|(N(1) \cap(\ell, k])^{-} \cup(N(1) \cap(k+1, n])^{-}\right| \\
& +\left|(N(1) \cap(\ell, k])^{-} \cap(N(1) \cap(k+1, n])^{-}\right| \\
< & s+t=\widetilde{\alpha}(G)+1 \leq \delta(G),
\end{aligned}
$$

a contradiction. This completes our final proof. 


\section{Summary}

This thesis contains a number of new contributions to the research field that studies the Hamilton problem, i.e., the problem of deciding whether a given graph admits a Hamilton cycle. Due to the NP-completeness of this problem, many graph theorists are focussing on finding sufficient conditions, i.e., conditions on the graph that guarantee the existence of a Hamilton cycle. The main topic of this thesis is exploring sufficient conditions for hamiltonicity of graphs, as well as for other hamiltonian properties. The results in this thesis mainly involve three types of conditions.

The first type of conditions is obtained by using the spectral radius. Given a graph, there are a variety of associated matrices. The spectrum of the graph, i.e., the (multi)set of eigenvalues of the corresponding matrix, can reflect some information of the structure of graphs. In Chapters 2-4 and 6-7, we present some sufficient conditions on the spectral radius for hamiltonian properties of graphs. The proofs of our main results are based on the Bondy-Chvátal closure, a degree sequence condition due to Chvátal, and the Kelmans transformation.

In Chapter 2, we focus on the hamiltonicity of graphs. We consider graphs with minimum degree $\delta(G) \geq k$, where $k \geq 1$. We give two sufficient conditions in terms of the spectral radius and the signless Laplacian spectral radius, respectively, for graphs to be hamiltonian. These two results both extend some previous analogues. To specify all the exceptional graphs, we also consider the special case when $k=1$ and obtain two corresponding results.

In Chapters 3-4, we focus on the Hamilton-connectivity of graphs. In Chapter 3 , we consider graphs with minimum degree $\delta(G) \geq 3$. We firstly 
obtain a weaker condition on the edge number for a graph to be Hamiltonconnected. Then by using it as a basis, we prove two results on the (signless Laplacian) spectral radius for the Hamilton-connectivity of a graph. Also, we obtain two corollaries from these two results, which show the extremal graphs with maximum spectral radius and maximum signless Laplacian spectral radius among all non-Hamilton-connected graphs. In Chapter 4, we consider the more general case that the graphs have minimum degree $\delta(G) \geq k$, where $k \geq 2$. We obtain a sufficient condition for graphs to be Hamiltonconnected in terms of the signless Laplacian spectral radius, which is a generalization of previous works.

In Chapters 6-7, we mainly deal with $k$-connected graphs. In Chapter 6, we present some new sufficient conditions in terms of the spectral radius for $k$-connected graphs to be hamiltonian or traceable, which extend the analogues of graphs with minimum degree $\delta(G) \geq k$. In Chapter 7, we obtain sufficient conditions based on the spectral radius for the Hamiltonconnectivity of $k$-connected graphs, which again extend the analogues of graphs with minimum degree $\delta(G) \geq k$.

The second type of conditions is obtained by using the Wiener index and the Harary index, which are two popular topological indices. Indeed, these two indices are widely studied and useful for characterizing the structure of a graph. In Chapter 5, we give some sufficient conditions on the Wiener and Harary indices for graphs to be Hamilton-connected or traceable from every vertex, in which the extremal graphs with minimum Wiener index and maximum Harary index among all non-Hamilton-connected or non-traceable from every vertex graphs are determined.

The last type of conditions is obtained by using the minimum degree. Degree conditions are the classic approach to the Hamilton problem and are still the most studied conditions. The basis of subsequent research in this field is the result given by G.A. Dirac in 1952, which states that if every vertex has degree at least half of the vertices of the graph, then it contains a Hamilton cycle. Motivated by this and the new definition of "bipartite hole number" proposed by McDiarmid and Yolov, in Chapter 8 we prove some sufficient conditions for a graph with a large minimum degree to be traceable or Hamilton-connected, thereby generalizing the analogues of Dirac's Theorem 
for traceable and Hamilton-connected graphs. Based on this, we also provide a sufficient condition for the existence of many edge-disjoint Hamilton paths in a graph. 



\section{Samenvatting}

Dit proefschrift bevat een aantal nieuwe bijdragen aan het onderzoeksgebied rondom het bestuderen van het Hamilton probleem, dat wil zeggen het probleem om te beslissen of een gegeven graaf een Hamiltoncykel bevat. Omdat dit probleem NP-volledig is, hebben veel grafentheoretici zich gericht op het vinden van voldoende voorwaarden, dat wil zeggen voorwaarden die garanderen dat er een Hamiltoncykel bestaat in grafen die aan die voorwaarden voldoen. Het centrale thema van dit proefschrift is het onderzoeken en ontwikkelen van nieuwe voldoende voorwaarden voor het bestaan van een Hamiltoncykel, of voor daaraan verwante hamiltonse eigenschappen. De resultaten van dit proefschrift betreffen hoofdzakelijk de volgende drie soorten voorwaarden.

Het eerste type voorwaarden berust op het gebruiken van de "spectral radius" van een graaf. Deze parameter geeft informatie over de eigenwaarden van een verscheidenheid aan matrices die corresponderen met de structuur van de graaf. In Hoofdstuk 2 tot en met 4, alsmede in Hoofdstuk 6 en 7 worden voldoende voorwaarden in termen van de "spectral radius" gepresenteerd voor hamiltonse eigenschappen van grafen. De bewijzen die we daar geven zijn gebaseerd op de Bondy-Chvátal afsluiting, een graadrijvoorwaarde van Chvátal, en de Kelmans-transformatie.

In Hoofdstuk 2 richten we ons op de existentie van een Hamiltoncykel in grafen met minimale graad $\delta(G) \geq k$, met $k \geq 1$. We geven twee voldoende voorwaarden in termen van de "spectral radius" en de "signless Laplacian spectral radius", respectievelijk, opdat de grafen hamiltons zijn. Deze twee resultaten zijn uitbreidingen ten opzichte van eerdere analoge resultaten. 
Om de uitzonderingsgrafen te karakteriseren kijken we ook naar het geval $k=1$, en leiden daarvoor twee overeenkomstige resultaten af.

In Hoofdstuk 3 en 4 richten we ons op de sterkere eigenschap dat de grafen Hamilton-samenhangend zijn. In Hoofdstuk 3 beschouwen we grafen met minimale graad $\delta(G) \geq 3$. We bepalen allereerst een zwakkere voorwaarde in termen van het aantal lijnen opdat een graaf Hamilton-samenhangend is. Met dit als basis bewijzen we twee resultaten voor het Hamiltonsamenhangend zijn in termen van de "(signless Laplacian) spectral radius". Tevens leidt dit tot twee gevolgen die duidelijk maken hoe de grafen met maximale "(signless Laplacian) spectral radius" eruit zien die niet Hamiltonsamenhangend zijn. In Hoofdstuk 4 bekijken we het meer algemene geval dat de grafen minimale graad $\delta(G) \geq k$ hebben, voor $k \geq 2$. Ook voor deze klasse van grafen bepalen we voldoende voorwaarden in termen van de "(signless Laplacian) spectral radius" voor het Hamilton-samenhangend zijn. Deze resultaten zijn generalisaties van eerder werk.

In Hoofdstuk 6 en 7 richten we ons voornamelijk op $k$-samenhangende grafen. In Hoofdstuk 6 presenteren we enkele nieuwe voldoende voorwaarden in termen van de "spectral radius" opdat $k$-samenhangende grafen een Hamiltoncykel of Hamiltonpad bezitten. Dit zijn uitbreidingen van de analoge resultaten voor grafen met minimale graad $\delta(G) \geq k$. In Hoofdstuk 7 bepalen we soortgelijke resultaten voor de eigenschap Hamilton-samenhangend voor $k$-samenhangende grafen. Ook dit zijn uitbreidingen van de resultaten die gebaseerd zijn op minimale graad $\delta(G) \geq k$.

Het tweede type voorwaarden is gebaseerd op de "Wiener index" en de "Harary index", twee populaire topologische parameters die uitgebreid bestudeerd zijn voor de karakterisering van de structuur van grafen, met name voor scheikundige toepassingen. In Hoofdstuk 5 geven we enkele voldoende voorwaarden betreffende de "Wiener index" en de "Harary index" voor de eigenschappen Hamilton-samenhangend en voor het bestaan van Hamiltonpaden die starten vanuit een willekeurig punt. Daartoe bepalen we tevens de extremale grafen met minimale "Wiener index" en maximale "Harary index".

Het laatste type voorwaarden heeft wederom te maken met de minimale graad. Graadvoorwaarden behoren tot de klassieke aanpak van het Hamilton 
probleem en zijn nog steeds de meest bestudeerde voorwaarden. Voor deze ontwikkelingen werd de basis gelegd door G.A. Dirac, die in 1952 bewees dat elke graaf waarin alle punten een graad hebben die tenminste de helft van het aantal punten is, een Hamiltoncykel bevat. Gemotiveerd door een betrekkelijk nieuwe definitie van het concept "bipartite hole number" afkomstig van McDiarmid en Yolov, leiden we in Hoofdstuk 8 een aantal voldoende voorwaarden af opdat grafen met een hoge minimale graad een Hamiltonpad hebben of Hamilton-samenhangend zijn. Onze resultaten zijn generalisaties van de analoge resultaten voor deze eigenschappen van de stelling van Dirac. We gebruiken onze resultaten ook om een voldoende voorwaarde af te leiden voor de existentie van meerdere lijndisjuncte Hamiltonpaden in een graaf. 



\section{Bibliography}

[1] M. Aouchiche, P. Hansen, Distance spectral of graphs: A survey, Linear Algebra Appl., 458 (2014) 301-386.

[2] V. Benediktovich, Sufficient spectral condition for Hamiltonicity of a graph, Linear Algebra Appl., 494 (2016) 70-79.

[3] C. Berge, Graphs and Hypergraphs, Translated from the French by Edward Minieka. Second revised edition. North-Holland Mathematical Library, Vol. 6. North-Holland Publishing Co., Amsterdam-London; American Elsevier Publishing Co., Inc., New York, 1976.

[4] J. A. Bondy, Basic graph theory: paths and circuits, in: Handbook of Combinatorics Volume I, Elsevier, Amsterdam, Lausanne, New York, Oxford, Shannon, Tokyo, 1995, pp. 3-110.

[5] J. A. Bondy, V. Chvátal, A method in graph theory, Discrete Math., 15 (1976) 111-135.

[6] J. A. Bondy, U. S. R. Murty, Graph Theory, Grad. Texts in Math, vol. 244, Springer, New York, 2008.

[7] H. J. Broersma, On some intriguing problems in hamiltonian graph theory - a survey, Discrete Math., 251 (2002) 47-69.

[8] A. E. Brouwer, W. H. Haemers, Spectra of Graphs, Springer-Verlag, 2011. 
[9] R. A. Brualdi, E. S. Solheid, On the spectral radius of complementary acyclic matrices of zeros and one, SIAM J. Algebra Discr. Methods, 7 (1986) 265-272.

[10] X. D. Chen, J. G. Qian, Sufficient conditions for Hamiltonian graphs in terms of signless Laplacian spectral radius, Linear and Multilinear Algebra, 66 (2018) 919-936.

[11] V. Chvátal, P. Erdős, A note on Hamiltonian circuits, Discrete Math., 2 (1972) 111-113.

[12] V. Chvátal, On Hamilton's ideals, J. Comb. Theory B., 12 (1972) 163168.

[13] P. Csikvári, On a conjecture of V. Nikiforov, Discrete Math., 309 (2009) 4522-4526.

[14] K. C. Das, B. Zhou, N. Trinajstić, Bounds on Harary index, J. Math. Chem., 46 (2009) 1369-1376.

[15] M. DeLeon, A study of sufficient conditions for hamiltonian cycles, Department of Mathematics and Computer Science, Seton Hall University, 2000.

[16] G. A. Dirac, Some theorems on abstract graphs, Proc. Lond. Math. Soc., 3 (1952), 69-81.

[17] A. A. Dobrynin, R. C. Entringer, P. Žigert, Wiener index of hexagonal systems, Acta Appl. Math., 66 (2001) 211-249.

[18] A. A. Dobrynin, I. Gutman, S. Klavžar, P. Žigert, Wiener index of hexagonal systems, Acta Appl. Math., 72 (2002) 247-924.

[19] P. Erdős, Remarks on a paper of Pósa, Magyar Tud. Akad. Mat. Kut. Int. Közl, 7 (1962) 227-229.

[20] G. H. Fan, New sufficient conditions for cycles in graphs, J. Combin. Theory, Ser. B, 37 (1984) 221-227. 
[21] R. J. Faudree, R. J. Could, M. S. Jacobson, R. H. Schelp, Neighborhood unions and Hamiltonian properties in graphs, J. Combin. Theory, Ser. B, 47 (1989) 1-9.

[22] R. J. Faudree, E. Flandrin, Z. Ryjáček, Claw-free graphs - a survey, Discrete Math., 164 (1997) 87-147.

[23] L. H. Feng, G. H. Yu, On three conjectures involving the signless Laplacian spectral radius of graphs, Publ. Inst. Math. (Beograd), 85 (2009) 35-38.

[24] L. H. Feng, X. M. Zhu, W. J. Liu, Wiener index, Harary index and graph properties, Discrete Appl. Math., 223 (2017) 72-83.

[25] M. Fiedler, V. Nikiforov, Spectral radius and Hamiltonicity of graphs, Linear Algebra Appl., 432 (2010) 2170-2173.

[26] J. Ge, B. Ning, Spectral radius and Hamiltonicity of graphs and balanced bipartite graphs with large minimum degree, Preprint available at arXiv:1606.08530v3.

[27] C. Godsil, G. F. Royle, Algebraic Graph Theory, New York (NY): Springer; 2001.

[28] R. Gould, Advances on the hamiltonian problem - a survey, Graphs and Combin., 19 (2003) 7-52.

[29] R. J. Gould, Recent advances on the hamiltonian problem: survey III, Graphs and Combin., 30 (2014) 1-46.

[30] R. J. Gould, Updating the hamiltonian problem - a survey, J. Graph Theory, 15 (1991) 121-157.

[31] Y. Hong , J. L. Shu, K. F. Fang, A sharp upper bound of the spectral radius of graphs, J. Comb. Theory B, 81 (2001) 177-183.

[32] R. A. Hore, C. A. Johnson, Matrix Analysis, Cambridge University Press, 1985. 
[33] H. B. Hua, B. Ning, Wiener index, Harary index and Hamiltonicity of graphs, MATCH Commun. Math. Comput. Chem., 78 (2017) 153-162.

[34] H. B. Hua, M. L. Wang, On Harary index and traceable graphs, MATCH Commun. Math. Comput. Chem., 70 (2013) 297-300.

[35] O. Ivanciuc, T. S. Balaban, A. T. Balaban, Reciprocal distance matrix, related local vertex invariants and topological indices, J. Math. Chem., 12 (1993) 309-318.

[36] K. Kawarabayashi, A survey on hamiltonian cycles, Interdiscip. Inform. Sci., 7 (2001) 25-39.

[37] A. K. Kelmans, On graphs with randomly deleted edges, Acta Math. Acad. Sci. Hung., 37 (1981) 77-88.

[38] H. Li, Generalizations of Dirac's theorem in hamiltonian graph theory - a survey, Discrete Math., 313 (2013) 2034-2053.

[39] R. Li, Laplacian spectral radius and some Hamiltonian properties of graphs, Appl. Math. E-Notes, 14 (2014) 216-220.

[40] Y. W. Li, Y. Liu, X. Peng, Signless Laplacian spectral radius and Hamiltonicity of graphs with large minimum degree, Linear and Multilinear Algebra, 66 (2018) 2011-2023.

[41] B. L. Li, B. Ning, Spectral analogues of Erdős' and Moon-Moser's theorems on Hamilton cycles, Linear and Multilinear Algebra, 64 (2016) 2252-2269.

[42] B. L. Li, B. Ning, Spectral analogues of Moon-Moser's theorem on Hamilton paths in bipartite graphs, Linear Algebra Appl., 515 (2017) 180-195.

[43] M. H. Liu, B. L. Liu, A survey on recent results of variable Wiener index, MATCH Common. Math. Comput. Chem., 69 (2013) 491-520.

[44] R. F. Liu, X. Du, H. C. Jia, Some observations on Harary index and traceable graphs, MATCH Commun. Math. Comput. Chem., 77 (2017) 195-208. 
[45] R. F. Liu, X. Du, H. C. Jia, Wiener index on traceable and Hamiltonian graphs, Bull. Aust. Math. Soc., 94 (2016) 362-372.

[46] R. F. Liu, W. C. Shiu, J. Xue, Sufficient spectral conditions on Hamiltonian and traceable graphs, Linear Algebra Appl., 467 (2015) 254-266.

[47] M. Lu, H. Q. Liu, F. Tian, Spectral radius and Hamiltonian graphs, Linear Algebra Appl., 437 (2012) 1670-1674.

[48] B. Lučić, A. Miličevicć, N. Trinajstić, Harary index - twelve years later, Croat. Chem. Acta., 75 (2002) 847-868.

[49] C. McDiarmid, N. Yolov, Hamilton cycles, minimum degree, and bipartite holes, J. Graph Theory, 86 (2017) 277-285.

[50] V. Nikiforov, Some inequalities for the largest eigenvalue of a graph, Comb. Probab. Comput., 11 (2002) 179-189.

[51] V. Nikiforov, Some new results in extremal graph theory, Surveys in Combinatorics 2011, Cambridge University Press, 2011, 141-181.

[52] V. Nikiforov, Spectral radius and Hamiltonicity of graphs with large minimum degree, Czechoslovak Math. J., 66 (2016) 925-940.

[53] B. Ning, J. Ge, Spectral radius and Hamiltonian properties of graphs, Linear and Multilinear Algebra, 63 (2015) 1520-1530.

[54] O. Ore, Hamiltonian connected graphs, J Math Pures Appl., 42 (1963) 21-27.

[55] O. Ore, Note on Hamiltonian circuits, Amer. Math. Monthly, 67 (1960) 55.

[56] D. Plavšić, S. Nikolić, Z. Mihalić, On the Harary index for the characterization of chemical graphs, J. Math. Chem., 12 (1993) 235-250.

[57] V. V. Prasolov, Polynomials, MTSNMO, Moscow, 2001.

[58] R. H. Shi, 2-Neighborhoods and hamiltonian conditions, J. Graph Theory, 16 (1992) 267-271. 
[59] J. Wei, Z. F. You, H. J. Lai Spectral analogues of Erdős' theroem on Hamilton-connected graphs, Applied Mathematics and Computation, 340 (2019) 242-250.

[60] H. Wiener, Structural determination of paraffin boiling points, J. Amer. Chem. Soc., 69 (1947) 17-20.

[61] K. X. Xu, K. C. Das, N. Trinajstić, The Harary index of a graph, Springer Briefs in Applied Sciences and Technology, Springer, Heidelberg, 2015, xvi+74 pp. ISBN:978-3-662-45842-6; 978-3-662-45843-3.

[62] K. X. Xu, N. Trinajstić, Hyper-Wiener indices and Harary indices of graphs with cut edges, Util. Math., 84 (2011) 153-163.

[63] L. H. Yang, Wiener index and traceable graphs, Bull. Aust. Math. Soc., 88 (2013) 380-383.

[64] G. D. Yu, Y. Z. Fan, Spectral conditions for a graph to be Hamiltonconnected, Applied Mechanics and Materials, 336-338 (2013) 23292334.

[65] G. D. Yu, M. L. Ye, G. X. Cai, J. D. Cao, Signless Laplacian spectral conditions for Hamiltonicity of graphs, J. Appl. Math., 2014, Art. ID 282053, 6 pp.

[66] B. Zhou, Signless Laplacian spectral radius and Hamiltonicity, Linear Algebra Appl., 432 (2010) 566-570.

[67] B. Zhou, X. Cai, N. Trinajstić, On the Harary index, J. Math. Chem., 44 (2008) 611-618.

[68] B. Zhou, H. H. Cho, Remarks on spectral radius and Laplacian eigenvalues of a graph, Czechoslovak Math. J., 55 (2005) 781-790.

[69] Q. N. Zhou, L. G. Wang, Distance signless Laplacian spectral radius and Hamiltonian properties of graphs, Linear and Multilinear Algebra, 65 (2017) 2316-2323. 
[70] Q. N. Zhou, L. G. Wang, Some sufficient spectral conditions on Hamilton-connected and traceable graphs, Linear and Multilinear Algebra, 65 (2017) 224-234.

[71] Q. N. Zhou, L. G. Wang, Y. Lu, Signless Laplacian spectral conditions for Hamilton-connected graphs with large minimum degree, Preprint available at arXiv:1711.11257.

[72] Q. N. Zhou, L. G. Wang, Y. Lu, Wiener-type invariants on graph properties, Filomat, 32 (2018), 489-502. 



\section{Acknowledgements}

This thesis is finally completed. Looking back on the past few years, the doctoral study is a long road with hardships and challenges, failure and success, tears and laughter. I was encouraged and supported by many people along the way. Now, I would like to take this opportunity to express my gratitude.

This first person I would like to thank is Professor Ligong Wang, my supervisor at NPU. In 2014, he brought me to the world of graph theory. Since then, I have been thinking I am a lucky person on the way of research. He is knowledgeable and rigorous with study. Under his guidance, I began learning graph theory, thinking about some problems independently and trying to start writing my first paper. Up to now, I still clearly remember how he corrected my first paper. He taught me the technique of writing papers and was so patient, even when there was an error every two lines in the introduction. He gave us enough freedom to choose our own favorite subject. He supported me to participate in many great conferences on graph theory and encouraged me to discuss with others, which extremely broadened my research horizons.

I would like to thank Professor Hajo Broersma, my supervisor at the UT. In study, he is full of wisdom and passion. I am amazed that he can quickly understand a complex proof and know the next step that I am going to do, which makes our weekly meetings more efficient. He has enough patience while revising our papers. He will notice every detail and make every part of the paper more reasonable and readable. In life, he is always earnest and humorous. I have worried about the life and study abroad for many times before I came here. I feel so lucky that he is my supervisor. I could get 
response and help from him if I had any problem, which made me feel like home and helped me get used to the new environment quickly. I learned a lot from him, not only his attitude to life and research, but also his kindness and integrity.

I would also like to express my thanks to Professor Shenggui Zhang of NPU. He organized many conferences in Xi'an, which gave me an important opportunity to know more graph theorists and broaden my research views. In addition, I want to thank Xiaogang Liu, a researcher full of passion. I can talk to him like friends and he can always give me some important suggestion.

Thanks must also go to my NPU colleagues. We went to climb mountain together, made delicious food together, discussed problems together ... so many beautiful memories I will never forget. It is because of you that my doctoral life becomes sweet and memorable.

My thanks also go to all FMT members at the UT. They are so friendly and enthusiastic, like a family. I greatly appreciate that I can join this happy family. I have received much support and help from them. I want to thank Ida. You are always warmhearted and helpful whenever I needed help. I will never forget the long email Yeray sent me encouraging me to come over tension before my presentation for the colloquium. Thank you so much. I could eat the cookies from Carlos every day. I enjoyed every interesting activity they organized. Those memories are so precious that can never be recaptured. I will put them in my heart forever.

I would also like to thank all of my friends for encouraging me, and giving me financial and time support. They can always be with me whenever I need. I also want to thank all my new friends I got to know in Enschede. We talked heart to heart. I am so lucky to know such interesting and heartwarming friends.

My greatest thanks go to my husband Yong Lu. Whenever I was upset with life or study, you were always there and tried to tell jokes to make me laugh. We share happiness and sorrow. We have a lot of sweet memories and I firmly believe we will be happier and happier in future.

My deepest thanks go to my family members, my parents and my sister. You give me infinite tolerance and love. You always believe me and support 
me to make every decision. You make me believe that we are the best and the most happiest family in the world.

Qiannan Zhou

July 2019, Enschede 



\section{About the Author}

Qiannan Zhou was born on November 27, 1992 in Huojia County, Xinxiang City, P. R. of China. From 1998 to 2001, she studied in an elementary school in her village. In 2002, her parents sent her to another primary school in the county to receive a better education. After the last two years of elementary education, from 2004 to 2007, she completed her middle school period in a boarding school. In 2007, she was accepted by Huojia No.1 High school, where she finished the senior high school education.

In September 2010, she started her study at Anyang Normal University. In June 2014, she obtained her bachelor degree. In September 2014, she became a graduate student at Northwestern Polytechnical University in Xi'an, under the supervision of Professor Ligong Wang. After receiving her master degree in June 2016, she was selected to become a PhD candidate in NPU with the same supervisor.

Starting from October 2018, she visited the group of Formal Methods and Tools, University of Twente, as a joint $\mathrm{PhD}$ student to perform research on the relationship between the spectral radius and hamiltonian properties of graphs, under the supervision of Professor Hajo Broersma. This research has been sponsored by the China Scholarship Council. 

In this thesis, we present a number of sufficient conditions for hamiltonian properties of graphs. In particular, we focus on degree conditions, spectral conditions, and Wiener index and Harary index conditions for traceability, hamil tonicity or Hamilton-connectivity of graphs. Our contributions extend existing results.

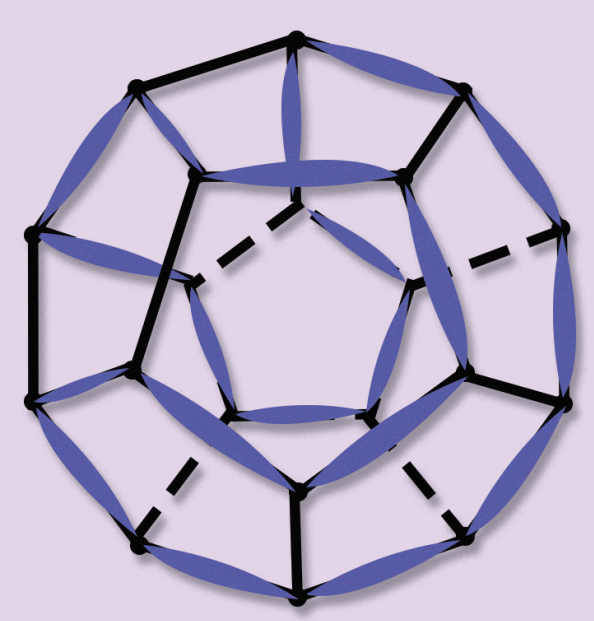

